\title{
Structural Transformations of Synaptic Partners During Growth and Competition of the Giant Nerve Terminal, the Calyx of Held, in the Medial Nucleus of the Trapezoid Body in the Developing Postnatal Mouse
}

Dakota Richard Jackson

West Virginia University, djacks14@mix.wvu.edu

Follow this and additional works at: https://researchrepository.wvu.edu/etd

Part of the Medicine and Health Sciences Commons

\footnotetext{
Recommended Citation

Jackson, Dakota Richard, "Structural Transformations of Synaptic Partners During Growth and Competition of the Giant Nerve Terminal, the Calyx of Held, in the Medial Nucleus of the Trapezoid Body in the Developing Postnatal Mouse" (2020). Graduate Theses, Dissertations, and Problem Reports. 7835. https://researchrepository.wvu.edu/etd/7835

This Dissertation is protected by copyright and/or related rights. It has been brought to you by the The Research Repository @ WVU with permission from the rights-holder(s). You are free to use this Dissertation in any way that is permitted by the copyright and related rights legislation that applies to your use. For other uses you must obtain permission from the rights-holder(s) directly, unless additional rights are indicated by a Creative Commons license in the record and/ or on the work itself. This Dissertation has been accepted for inclusion in WVU Graduate Theses, Dissertations, and Problem Reports collection by an authorized administrator of The Research Repository @ WVU. For more information, please contact researchrepository@mail.wvu.edu.
} 
Structural Transformations of Synaptic Partners During Growth and Competition of the Giant Nerve Terminal, the Calyx of Held, in the Medial Nucleus of the Trapezoid Body in the Developing Postnatal Mouse

\title{
Dakota Richard Jackson
}

\author{
Dissertation submitted \\ to the Rockefeller Neuroscience Institute at West Virginia University \\ in partial fulfillment of the requirements for the degree of \\ Doctor of Philosophy in \\ Neuroscience
}

Bernard Schreurs, Ph.D., Chair

Visvanathan Ramamurthy, Ph.D.

Candice Brown, Ph.D.

Peter Mathers, Ph.D

George Spirou, Ph.D., Mentor

Department of Neuroscience

Morgantown, West Virginia

2020

Keywords: neuron, development, synapse, spine, collateral, MNTB, calyx Copyright 2020 Dakota Richard Jackson 


\title{
Abstract \\ Structural Transformations of Synaptic Partners During Growth and Competition of the Giant Nerve Terminal, the Calyx of Held, in the Medial Nucleus of the Trapezoid Body in the Developing Postnatal Mouse
}

\author{
Dakota Richard Jackson
}

Across many parts of the nervous system, initial neural circuit assembly involves stereotyped stages of early axonal proliferation within target territories, selective reinforcement of a subset of inputs and the removal of exuberant or unneeded connections via either activity or experiencedependent mechanisms, in a process referred to as synaptic competition. The mechanisms and associated structural transformations that underlie and define these stages of neurodevelopment have been examined in a variety of brain regions including the peripheral neuromuscular junction, the central climbing fiber innervation of cerebellar Purkinje cells and retinal ganglion cell innervation of the thalamic lateral geniculate nucleus. Each of these connections are considered representative examples of sites of synaptic competition. We and others have established the globular bushy cell (GBC) innervation of principal cells (PC) of the medial nucleus of the trapezoid body (MNTB) in the auditory brainstem via the large calyx of Held $(\mathrm{CH})$ as an additional representative, or model, system for the study of terminal growth and competition.

We utilized dense reconstruction of pre and postsynaptic elements from serial block-face scanning electron microscopy (SBEM) image volumes collected during key developmental timepoints from both prehearing (postnatal days $(\mathrm{P}) 2,3,4,6, \& 9)$ and adult (P30) mice. In this document, I report our most recent studies of MNTB ultrastructure, which revealed additional structural elements that define the progressive growth, competition and subsequent maturation of the $\mathrm{CH}$ and its innervation of PCs.

We found evidence that all developing $\mathrm{CHs}$ extend 3 types of collateral arbors including short filopodia $(<5.85 \mu \mathrm{m})$, long filopodia $(>5.85 \mu \mathrm{m})$, and growth cone tipped collaterals of sufficient length to innervate adjacent cells or cells within $60 \mu \mathrm{m}$, but in some cases up to $100 \mu \mathrm{m}$, away from the innervation territory of the $\mathrm{CH}$. Moreover, as the proportion of polyinnervated PCs with competing $\mathrm{CH}$ inputs declines from $23 \%$ at $\mathrm{P} 6$ to $11 \%$ at $\mathrm{P} 9$, we found a significant reduction in the length of $\mathrm{CH}$ collaterals until few if any were found by maturity (P30).

Using the first live imaging data collected from a living brain slice with the lattice light sheet microscope, we revealed with high spatial and temporal resolution that $\mathrm{CH}$ collaterals exhibited some of the fastest motility recorded in developing neural systems (average growth cone rate of motility: $2.1 \mu \mathrm{m}$ per min). We also observed interactions among long filopodial collaterals that resulted in retraction and altered growth trajectories suggesting possible repulsive contactmediated guidance of $\mathrm{CH}$ growth and innervation. These may be the predominant locations where competing CHs physically interact, since they typically expand over non-overlapping territories across the cell surface of the PC soma. 
Postsynaptically, we provided the first systematic description of two types of invaginating spine (type 1 and 2) that reside specifically within recesses underneath and penetrating the developing $\mathrm{CH}$. Similar to the emergence of $\mathrm{CH}$ collaterals, we found a strong association of type 1 spine emergence with the initial expansion of the terminal. Moreover, for the first time, somatic spine mats, composed of so-called type 2 spines that intertwine to assemble a macrostructure called the somatic spine mat, were described in the vertebrate CNS. From inspection of EM images, we suggest three modes of intercellular communication between the $\mathrm{CH}$ and its synaptic partner, the PC: chemical synaptic transmission, signaling via cell adhesion proteins, trans-endocytosis of spine mats into the $\mathrm{CH}$.

We hypothesize that the unifying event to synchronize $\mathrm{CH}$ growth and emergence of collaterals and postsynaptic somatic processes is a transformation of spontaneous activity to a bursting pattern. Preliminary experiments reveal that interference with synaptic transmission using viral vector induced expression of tetanus toxin leads to morphological changes in the $\mathrm{CH}$ and slower growth of the postsynaptic cell body. Further experiments are proposed that combine electrophysiology and dynamic imaging with molecular and physiological perturbation to further explore these linkages. 


\section{Dedication}

For my family: Having your unwavering support and encouragement is what has facilitated my entire education. 


\section{Acknowledgements}

\section{To George Spirou,}

As my mentor and research advisor, you have dedicated a tremendous amount of time directly into my training and education which I will carry with me for the remainder of my career.

To my lab partners, Ashley, Paul, Dan and Michael, your rich backgrounds and unrivaled enthusiasm has made working the lab so much more fruitful and exciting!

To my research committee,

Thank you for your insights and efforts in guiding the direction my research has taken. 


\section{Table of Contents}

\section{Chapter 1: Introduction and Literature Review}

Early Neural development and circuit formation .............................................. 1

Axon Formation ................................................................ 2

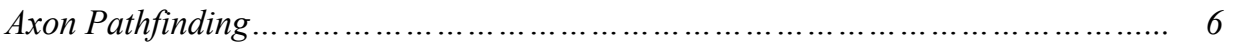

Synaptic Exuberance and Pruning..................................................... 10

The Neuromuscular Junction ............................................ 11

Climbing Fiber Innervation of Purkinje Cells ............................. 14

Retinal Ganglion Cell Innervation of the

Dorsal Lateral Geniculate Nucleus................... 16

Calyx of Held Innervation of the

Medial Nucleus of the Trapezoid Body............ 19

Development of the Calyx of Held .................................................... 20

Role of the Calyx of Held in Auditory Processing....................................... 22

Initial Genesis of the Auditory Brainstem ............................................... 23

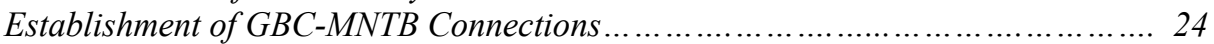

Formation of a Large Terminal ......................................... 25

Morphology of the Protocalyx ......................................... 27

Transient Period of Synaptic Competition................................. 29

Early Stage Strengthening and Pruning............ 30

Late-Stage Strengthening and Pruning............. 31

Maturation and Fenestration of the $\mathrm{CH}$................................. 32

Maturation of the Postsynaptic Principal Cell........................... 33

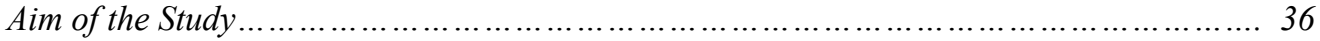

\section{Chapter 2: Rapid Terminal Dynamics Within Target Territories}

\section{During Neural Circuit Formation.}

Introduction ....

Results

\section{Collateral Number and Length Increase Transiently} during Nerve Terminal Growth....................... 41

Arrangement of CH Collaterals Facilitate Selective Spatial Overlap....... 42

Internal Structure and Contacts of CH Collaterals............................. 43

Dynamic Imaging of the Developing MNTB

Calyx Collaterals 45

Short Filopodial Collaterals......................................... 46

Long Filopodial Collaterals ....................................... 48

Long Growth Cone Collaterals ......................................... 49

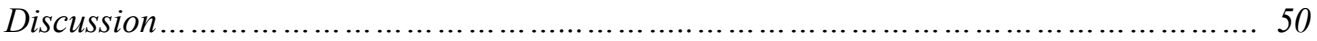

Dynamic Tissue Reorganization ................................................ 51

Peak Dynamics of Calyx Growth ................................................. 53

Critical Period for CH:MNTB Formation ……................................ 54

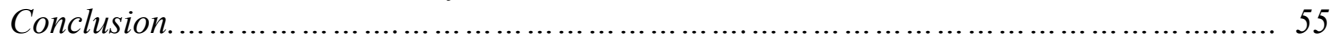


Materials and Methods

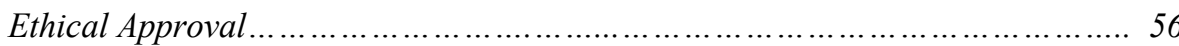

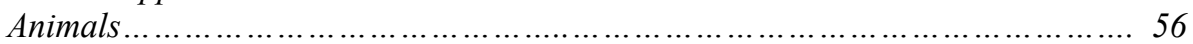

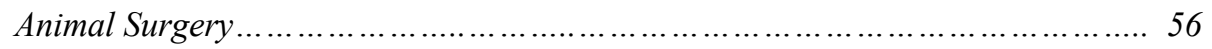

Live-Slice Preparation and LLS Imaging ............................................. 57

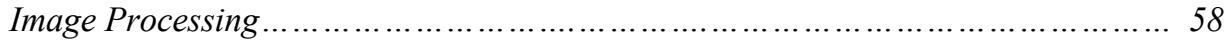

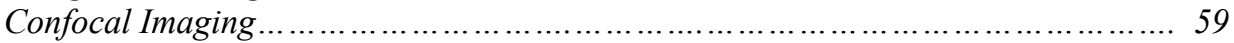

Serial Block-Face Scanning Electron Microscopy …..................................... 59

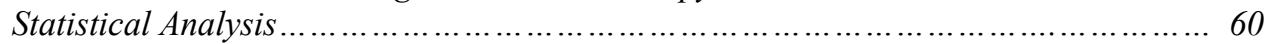

\section{Chapter 3: Two Types of Somatic Spines Provide Sites for Intercellular \\ Signaling During Calyx of Held Growth and Maturation............}

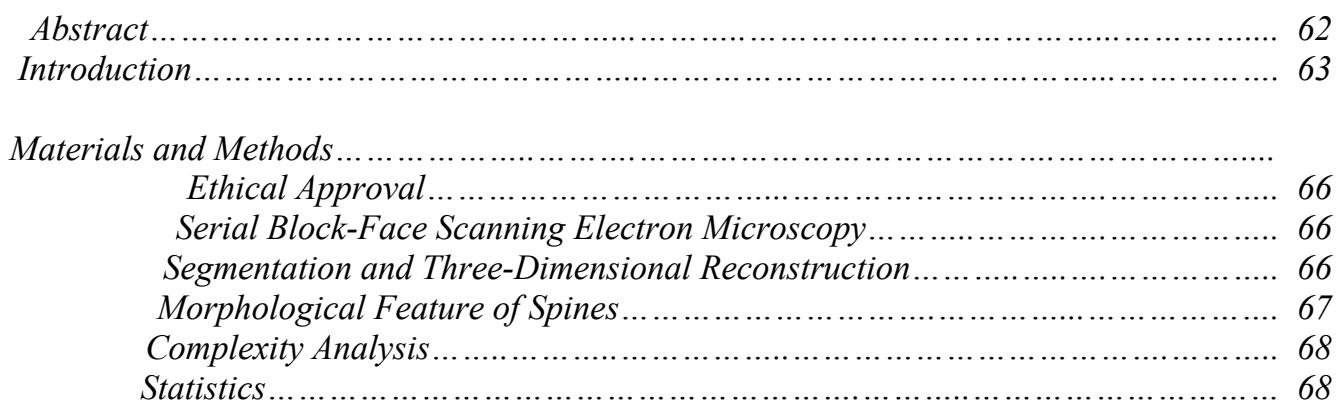

Results.......

The Shortest Somatic Process Are Located Under the Calyx of Held................ 70

Somatic Processes Underlying the CH Are Classifies Into Two Groups............ 70

Prevalence of Type 1 Somatic Spines and Spine Mats is Developmentally Regulated .............................. 73

Type 1 Spine and Spine Mat Prevalence Do

Not Differentiate Competing Inputs...................... 75

Types 1 and 2 Spines Provide Area for Membrane Apposition and are Sites for Innervation................... 76

Trans-Endocytosis of Spine Mats Into the Presynaptic Terminal ....................... 78

Discussion.

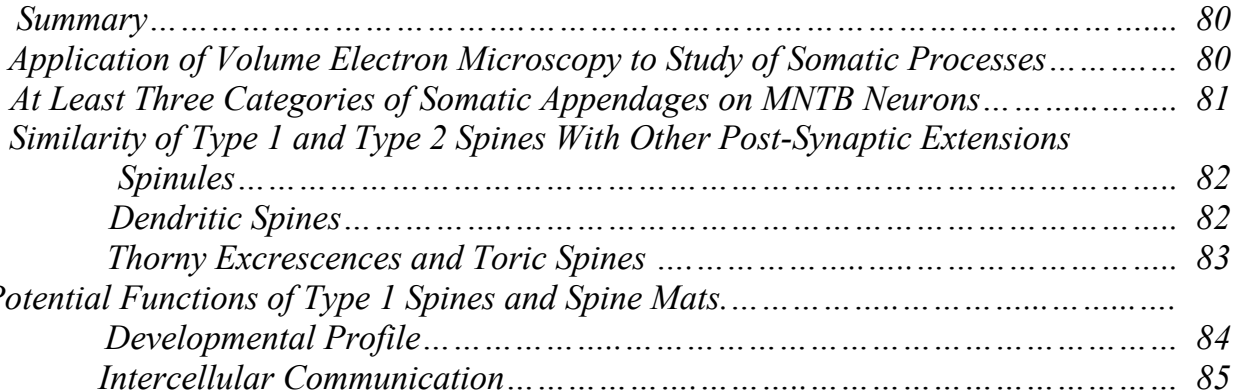

Chapter 4: Role of Spontaneous Activity on the Growth of the CH ......... 
Introduction

Methods.....

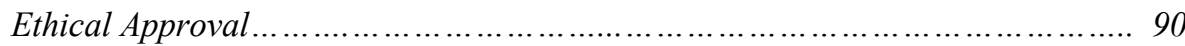

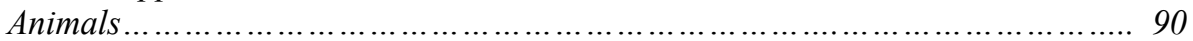

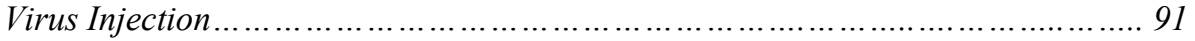

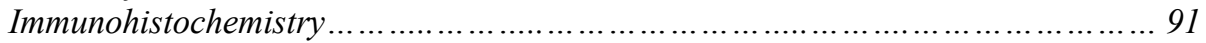

Segmentation and Three-Dimensional Reconstruction ............................ 92

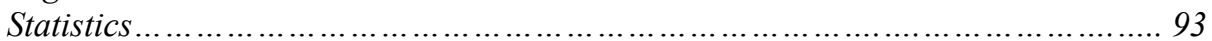

Results

Constitutive GFP-tox Expression in the Developing Auditory System....... 93

Heterogeneous tox Expression in the Developing Auditory Brainstem........ 95

Discussion.

97

\section{Chapter 5: Discussion and Future Directions}

Elaboration of CH Collateral Arborization.............................................. 102

Dynamic Motility and Potential Function of $\mathrm{CH}$ Collaterals ........................ 106

Postsynaptic Specialization During Growth of the CH.............................. 109

Population Dynamics And The Persistence of CH Collaterals

And Somatic Spines....................................... 110

Signaling Capacity of Type 2 Spines................................................... 112

Role of Neurotransmission in the Establishment of the CH........................ 114

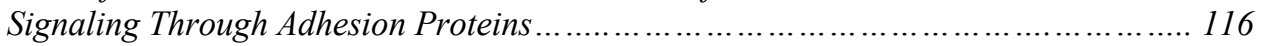

Signaling Via Trans-Endocytosis of Spine Mats.................................... 116

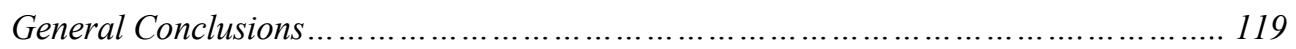

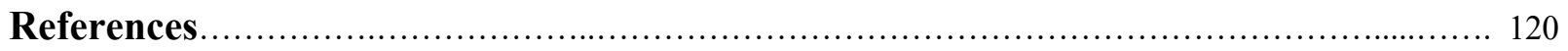




\section{Chapter 1}

\section{Introduction and Literature References:}

\section{Early Neural Development and Circuit Formation}

The nervous system is composed of a complex and highly organized assemblage of specialized cells called neurons, capable of rapidly transmitting electrochemical signals necessary for processing sensory stimuli, memory storage, and coordinating the planning and execution of actions in response to environmental challenges. In order for the nervous system to produce the large variety of requisite biologic computations, diverse subpopulations of neurons exhibit unique differences in structure and connectivity that arise following a complex mode of intrinsic and extrinsic regulation of gene transcription during development (Pearson and Doe, 2004). An important question that has yet to be answered satisfactorily, however, is how the hundreds (C. elegans)(White et al., 1986) to billions (roughly 86 billion neurons in the human brain (Azevedo et al., 2009)) of neurons autonomously assemble in order to provide a substrate for processing all the implicit functions of sensation, perception and intention (Azevedo et al., 2009).

The formation of a mature neural network requires a concerted sequence of events, including the production of new cells, differentiation and migration of neurons, and the generation of functional and specific synaptic connections between neurons. Mice offer advantages to understanding brain development in mammals, due to their small size and genetic and structural similarities to primates, including humans (Mural et al., 2002; Bryda, 2014; Snyder et al., 2018). Moreover, their shorter gestation period of 18-21 days requires many of the same developmental processes to occur in a more compressed timeframe relative to humans 


\section{Chapter 1}

(Murray et al., 2010). In mice, formation of the neural plate begins around embryonic day 7 (E7) and neurulation occurs between E8.5-10 (Kaufman, 1992). Thus, many of the $\sim 100$ million neurons in the mouse brain are largely formed during an approximate 11-day period of late embryonic development, although some neurogenesis continues into the early postnatal (P) period (De Felipe et al., 1997).

\section{Axon Formation}

Following neurogenesis, cells must begin to take on the polarized morphology indicative of a neuron. A key question is what determines how axons find appropriate sites to deliver their electrochemical signals? Moreover, what mechanisms determine if these cells will produce hundreds to thousands of synaptic contacts, which can reach a density of about one trillion synaptic connections per cubic centimeter of tissue in cortical tissue (Drachman, 2005)? These questions were initially proposed over a century ago by Santiago Ramón y Cajal (Ramón y Cajal, 1892) and partially answered by his fastidious work with embryonic histological sections. From his study of fixed tissues, Ramón y Cajal described observations of neural processes tipped with a complex structure called a growth cone, characterized by an expanded lamellipodium often studded with finger-like filopodia (Ramón y Cajal, 1890). Ramón y Cajal realized these structures were largely absent in the more developed cells of the mature brain and such an observation likely helped spur his hypothesis that growth cones were influential in the development of the brain (de Castro et al., 2007). In more recent years, a clearer picture of neural development has emerged largely from work with dissociated and acute tissue cultures using methods that allow cells in vitro to survive for periods sufficient to observe the sequential stages 


\section{Chapter 1}

of neuron development (Dotti et al., 1988; Rodríguez-Hernández et al., 2014; JedrzejczakSilicka, 2018).

Among the first descriptions of the process of structural neural differentiation came from the study of dissociated, post-mitotic hippocampal neurons (Dotti et al., 1988; Goslin and Banker, 1989). Here it was found that multiple short, appendages tipped with growth cones and interstitial filopodia bud from spherical neurons within a day of dissociation and culture. However, among these short appendages, key differences could be observed. First, a single neurite with a 'smooth and uniform' morphology was shown to elongate up to five times more quickly than others, at up to $60 \mu \mathrm{m} /$ day (12 $\mu \mathrm{m} /$ day for dendrites) (Dotti et al., 1988). Moreover, once a single neurite achieved a length greater than $10 \mu \mathrm{m}$ relative to others, it could be reliably identified as an axonal extension. This process was further distinguished from the 'branched and tapered morphology of the more slowly elongating dendrites (Dotti et al., 1988; Goslin and Banker, 1989). In part, these differences in neurite motility are likely driven by differences in underlying cytoskeletal structure and dynamics.

The major structural elements fundamental to aiding in the development of neurites are comprised of actin and tubulin. In brief, actin filaments and microtubules are formed from ATPbound actin monomers and GTP-bound a- $\beta$-tubilin heterodimers to form long polymer chains, respectively. The elongation of polymer chains occurs primarily in a polarized fashion through addition of subsequent subunits to the "barbed end" of actin filaments (Pollard and Cooper, 2009) or the "plus-end" of microtubules at the exposed $\beta$-subunit (Chretien et al., 1992). Actinenriched areas of an axon, such as dynamic and planar lamellipodia that tip growing axons and are often studded with thin projections called filopodia, are driven to elongate through mechanical forces exerted by extending actin filaments (Blanchoin et al., 2014). In filopodia, 


\section{Chapter 1}

parallel associations of enriched actin filaments collectively orient their barbed ends driving elongation towards the cell membrane to extend the tip of the process. In lamellipodia, however, actin filaments are arranged in a web-like orientation driving filament elongation in various directions leading to a non-uniform expansion of the veiled lamellar structure. Microtubules, meanwhile, are enriched in the main process of the axons and dendrites although the arrangement differs significantly between them (Barnes and Polleux, 2009). Axons exclusively exhibit a uniform and polarized arrangement of microtubules such that active polymerization occurs unilaterally at the distal plus-end of axonal microtubule bundles (Athamneh et al., 2017). Meanwhile, dendrites exhibit a more disorganized arrangement of microtubules wherein the majority of microtubule polymerization occurs at intermediary points along the length of a maturing dendrite without significant bias towards the somatic or distal end of the dendrite (Baas et al., 1988).

Long-distance axonal pathfinding is thought to be mediated by a lammelipodial-like structure termed a growth cone (Lowery and Van Vactor, 2009). The process of growth cone extension recapitulates the initial steps in process formation. During the initial formation of neuronal processes, a small cellular protrusion forms along the edge of the cell membrane. This early process is invaded by elongating actin filaments that generate the required force (up to 3 $\mathrm{pN}$ ) to extend the cell membrane to form thin filopodial structures (Torre et al., 2007). In order to stabilize these filopodia, microtubules must invade previously actin-enriched areas. A similar process is responsible for branch formation in neurites as the initial actin-mediated extension of short filopodia is followed by subsequent invasion of microtubule bundles that lengthen and stabilize collateral branches (Gallo, 2011). Moreover, interaction of actin filaments and microtubules is perhaps best illustrated in the cytoskeletal arrangement and dynamics within 


\section{Chapter 1}

neuronal growth cones. Growth cones are composed of two primary domains: a peripheral domain (P-domain) that encompasses radial filopodia and a veiled lamellipodium each composed primarily of dynamic actin filaments, and a central domain (C-domain) with a cytoskeleton predominated by microtubules that extend from the axon to the edge of the central domain near the base of the growth cone lamellipodium (Figure 1)(Geraldo and Gordon-Weeks, 2009; Lowery and Van Vactor, 2009). In order for a growth cone to advance forward, microtubules must first invade territory previously occupied by actin in the P-domain (Sabry et al., 1991). Then, microtubules in the C-domain of the growth cones are bundled and tubulin is preferentially recruited to the plus-end of the polymer at the base of the growth cone which advances the position of the central domain of the growth cone and allows the distal axon segment to extend (Tanaka et al., 1995). Importantly, the recruitment of new tubulin molecules necessary for sustained microtubule elongation in axon growth requires transport of new constituent cytoskeletal molecules from elsewhere in the neuron along the length of the axon via molecular motors. This conclusion was initially based on seminal studies of axonal transport that utilized radioisotopic pulse labeling techniques. These experiments provided evidence for at least two modes of transport, a fast (100-400 mm/day) and slow (1-10 mm/day; (Brown, 2003; Roy et al., 2005)) component, were required to fully explain the observed kinetics of axonal cargos (Grafstein and Forman, 1980). Non-membrane bound cytoskeletal elements such as tubulin are transported rapidly over short distances at average rates of $\sim 1 \mu \mathrm{m} / \mathrm{s}(\sim 86 \mathrm{~mm} /$ day $)$ with average peak velocities of $\sim 2 \mu \mathrm{m} / \mathrm{s}(\sim 173 \mathrm{~mm} /$ day) (Wang and Brown, 2002). Thus, the dynamics of actin and microtubule polymerization/depolymerization are rapid and must be tightly regulated through a myriad of interacting proteins that coordinate cytoskeletal interactions and control 


\section{Chapter 1}

neurite morphology during rapid neurite extension and motility (Dent et al., 2011; Dogterom and Koenderink, 2019).

\section{Axon Pathfinding}

Soon after his initial description of the growth cone (Ramón y Cajal, 1890), Ramón y Cajal referred to then current cell biologic evidence from other systems including microbiology as he theorized the growth cone could provide a mode of chemotactic guidance. Cajal suggested growth cones utilize secretions from other cells in the nervous system in a fashion that mirrored the contemporary findings of Pfeffer who was carefully describing and providing evidence for chemotaxic behaviors in cells from bacteria to leukocytes and even fern spermatozoids (Pfeffer, 1884, 1888; Ramón y Cajal, 1892). Amazingly, Ramón y Cajal made these predictions without directly observing a living growth cone, a feat that would not be achieved until neuronal tissue culturing became possible in 1910 (Harrison, 1910). This forward thinking "neurotropic hypothesis" Ramón y Cajal proposed failed, however, to incorporate the idea that repellant signals too importantly aid in accurate axonal pathfinding. This was addressed later by Roger Sperry who provided the more refined "chemoaffinity hypothesis" that suggested the molecular identity of an axon was useful in identifying attractant or repellant signals that guide its direction of growth and to some degree aid in regrowth after injury through local detection of graded chemical cues or contact-mediated communication via expression of receptors found on a growth cone (Sperry, 1963). In the decades since, Sperry’s hypotheses have been validated through the identification of many of the membrane-bound receptors found enriched along growth cones and 


\section{Chapter 1}

filopodia and their associated ligands expressed during development (Harris et al., 1987; Sanes and Yamagata, 2009; Williams et al., 2010).

Neuronal guidance factors can be subdivided into at least four highly conserved families of ligand and associated receptors including: 1) netrins/DCC/UNC5 (Sun et al., 2011), 2) slits/Robos (Wong et al., 2002), 3) ephrins/Ephs (Klein, 2004) and 4) semaphorins/plexins (Tamagnone and Comoglio, 2000) although several morphogens (BMPs, Shh, Wnts and others) may additionally serve as attractive or repulsive cues for axonal guidance as well (Zou and Lyuksyutova, 2007). These cues can be encountered by axons when found along a diffusional gradient diffusely spread across the extracellular environment, integrated into components of the extracellular matrix environment and/or incorporated into intermediary cell surfaces (TessierLavigne and Goodman, 1996; Huber et al., 2003; Mortimer et al., 2008; O’Donnell et al., 2009; Chédotal and Richards, 2010; Kolodkin and Tessier-Lavigne, 2010). Virtually all guidance molecules mediate the regulation of the structure and stability of the underlying cytoskeleton, especially targeting actin within the motile peripheral domain of the growth cone, via a variety of molecular mechanisms including activation of Rho family GTPases that further activating additional effectors such as kinases and actin binding proteins (Song and Poo, 1999; Suter and Forscher, 2000; Luo, 2002; Koh, 2007; O’Donnell et al., 2009). However, a second welldescribed effect of guidance receptor activation is exerted through induction of $\mathrm{Ca}^{2+}$-dependent signaling that indirectly regulates membrane recycling through influences in the balance of endoand exocytosis that preferentially extend the membrane of the growth cone toward chemoattractants and away from chemorepellants (Song and Poo, 1999; Tojima et al., 2014). For a more comprehensive review of general mechanisms of axon guidance, refer to the following reviews (Raper and Mason, 2010; Gomez and Letourneau, 2014). 


\section{Chapter 1}

Netrin-1 was first purified from the brain tissue of chicks and found to effectively guide elongating commissural axons as an attractant signal towards the floor plate of the developing spinal cord (midline of the neural tube) in in vitro studies (Serafini et al., 1994, 1996). DCC mediated growth towards gradients of secreted netrin-1 was found to be a potent attractant signal for retinal ganglion cells exiting the retina through the optic nerve head (de la Torre et al., 1997). Alternatively, expression of UNC-5, another netrin-1 receptor repels axons outgrowth (Chan et al., 1996; Keleman and Dickson, 2001). Co-expression of DCC and UNC5 allows the membrane-bound proteins to bind about their cytoplasmic tails, downregulating DCC and converting the effect of DCC-mediated guidance to UNC-5/DCC mediated repulsion (Hong et al., 1999; Keleman and Dickson, 2001).

A study utilizing forward genetic screens in Drosophila identified over 250 genes with possible implications in axon guidance (Seeger et al., 1993). Importantly, mutations in two of these identified genes produced inverse routing errors during axonal pathfinding at the midline: mutated roundabout (Robo) receptors produced aberrant contralateral projections; commissureless (Comm) induced severe deficits in commissural axon pathfinding as axons failed to cross the midline (Seeger et al., 1993). Slit, a large extracellular matrix protein produced by midline glial cells in drosophila was later confirmed as a chemo-repulsive ligand for the growth cone-bound Robo receptor (Kidd et al., 1999) and has been found to be an evolutionarily conserved signal utilized from invertebrate to mammalian development (Brose et al., 1999). In addition to elongating axons, Comm is also expressed by cells at the midline. Paradoxically, axons that need to cross the midline are inhibited by the presence of slit-mediated repulsion. In order to overcome this effect, a second gene (Comm) expressed by and concentrated along midline glia downregulates the expression of Robo in commissural axons mitigating the 


\section{Chapter 1}

repulsive signaling of slit proteins during their passage across the central commissure. Then, as axons reach the contralateral side of the brain, where Comm expression is reduced, Robo is again upregulated to reinstitute a slit-mediated repulsion (Kidd et al., 1999; Georgiou and Tear, 2002). Binding of slit to Robo induces a conformational change in the membrane-anchored Robo protein that primes the receptor for metalloprotease-mediated cleavage, subsequently altering the signaling capacity of the receptor and preventing recurrent attraction towards the midline (Coleman et al., 2010).

Ephrins represent a third family of guidance molecule and are comprised of two classes: Ephrin-A that is anchored to the membrane through a GPI anchor and ephrin-B that also is linked through a transmembrane protein with a short cytoplasmic PDZ-binding domain (Wilkinson DG, 2001). Many Ephrins, uniquely among guidance molecules, can undergo bidirectional signaling through interaction with their respective tyrosine kinase receptors, Eph-A and Eph-B, a phenomena extensively reviewed elsewhere (Egea and Klein, 2007). Generally, ephrin signaling promotes repulsive growth cone behaviors following cell-cell interaction such as occurs in the commissural migration of retinal ganglion cell axons (Nakagawa et al., 2000) although some cases of attractive behaviors have been documented such as occurs following ephrin association with the Ret tyrosine kinase receptor in developing motor axons (Bonanomi et al., 2012).

The fourth major family of guidance molecule, semaphorins, can function as both diffusible or contact-mediated cues that bind to an array of surface receptors, primarily through interaction with plexin molecules (Perälä et al., 2012), to form multimeric protein complexes that generally serve as a repulsive signal during axonal migration (Mann et al., 2007). However, the effects of semaphorins can be complicated as their co-receptor, neuropillins (Nrp), generate attractant signaling. Thus, the effect of semaphorin-mediated signaling represents a balance in 


\section{Chapter 1}

the expression of particular guidance receptors in axons in order to reach appropriate terminal locations. Such a balance is exemplified in the graded and overlapping expression of EphA5 and Nrp1 among others, that must be sensed by axons arriving within the developing olfactory bulb (Imai et al., 2009; Schwarting and Henion, 2011).

\section{Synaptic Exuberance and Pruning}

The chemoaffinity hypothesis only partially explains the assembly of the nervous system. As axons reach their distal target nuclei, target-derived signals (including traditional guidance molecules, neurotrophins and cell-adhesion molecules) initiate a behavioral change in the axon that induces altered growth cone dynamics (including prolonged pauses in motility and expansion of the growth cone) and an elaboration of the axon through interstitial collateral branch formation (Szebenyi et al., 1998; Krylova et al., 2002; Bak and Fraser, 2003; Gutierrez et al., 2005; Bilimoria and Bonni, 2014). Often, such divergence of an axon produces supernumerary afferent projections that connect it to more target neurons than are maintained in the mature connectome, suggesting early exuberance must be followed by subsequent synapse elimination in order to further increase the specificity of neuronal connections (Lohof et al., 1996; Hensch, 2004; Schuldiner and Yaron, 2014). For example, as many as a $25-50 \%$ of synaptic connections in the neonatal human brain are pruned by puberty in a process that refines the mature connectome and underlies the maturation and consolidation of cognitive faculties (Huttenlocher, 1979; Huttenlocher and de Courten, 1987). Generally speaking, such progressive and selective elimination of excessive innervation has been described as a process of "synaptic competition" in which afferents "compete" for limited availability of local resources, territory or 


\section{Chapter 1}

relative synaptic competency (Bi and Poo, 2001; Buffelli et al., 2003; McCann et al., 2007; Davies, 2008). Synaptic competition has been demonstrated in a variety of brain regions, especially sensory systems that undergo a sensitive period of development in which an initial innervation within a circuit must be honed into an ever more precise pattern of connectivity (Hensch, 2004), including but not limited to: cortical targets of thalamocortical projections to the somatosensory cortex (Rios and Villalobos, 2004), corticofugal projections (Curfs et al., 1995), visual cortex (Innocenti et al., 1977) as well as within the thalamus (Hong and Chen, 2011), brainstem nuclei (Holcomb et al., 2013), and in the cerebellum (Watanabe and Kano, 2011). Perhaps the most well-studied area of the nervous system exemplifying synaptic competition among exuberant presynaptic innervation occurs in the peripheral motor end plate along muscle fibers by motor neurons at the so-called neuromuscular junction, or NMJ (Wu et al., 2010; Tintignac et al., 2015). The following sections will outline the stages of development culminating in targeted synaptic refinement that have been identified in several key brain regions.

\section{The Neuromuscular Junction}

Work in developing systems with ultimate 'one-to-one' or 'few-to-one' connectivity offers a clear advantage in investigating the selective strengthening and pruning events that sculpt immature circuits during competition; the endpoint of competition is more readily identifiable in these systems. In part, the NMJ has become one of the most utilized systems for studying synaptic competition because of its large size and readily accessible location in the periphery. These features make it amenable to both in vitro and in vivo electrophysiologic 


\section{Chapter 1}

(Colman et al., 1997; Favero et al., 2007; Vilmont et al., 2016), genetic (Buffelli et al., 2003) and microscopic investigation (Ball et al., 2003; McCann and Lichtman, 2008) in a variety of species from drosophila to vertebrates (Lu and Lichtman, 2007; Wu et al., 2010). As myogenesis occurs during embryonic development each newly formed motor endplate is prepatterned with dense assemblages of acetylcholine receptors (AChR) (Zhang et al., 2009). In mice, by the day of birth, collections of motor neurons arrive and as many as 10-20 unique afferents have formed small bouton-sized terminals (Brown et al., 1976; Tapia et al., 2012). During postnatal development in this system, two distinct phases of competition resolve supernumerary innervation of the NMJ such that a single motor neuron provides dominant monoinnervation across the entire endplate. First, a subset (2-3) of the initial pool of 10-20 motor neurons increase in size and other inputs withdraw from the motor endplate (Bishop et al., 2004). Secondly, during a protracted period that may last for up to two weeks of postnatal development, the remaining inputs then compete until a single input predominates each endplate (Brown et al., 1976; Balice-Gordon and Lichtman, 1993; Walsh and Lichtman, 2003).

The process of selective refinement does not occur randomly at the developing motor endplate, but instead requires synaptic transmission wherein the most active inputs, as defined by either the frequency of synaptic release or the net total secreted neurotransmitter, aids in the establishment of dominance. This principal requirement of synaptic activity was beautifully demonstrated via in vivo experiments where it was shown that inputs indirectly compete such that less active inputs retreat and vacated sites along the motor endplate can be overtaken by more active inputs (Turney and Lichtman, 2012). Moreover, the requirement of synaptic activity in selective refinement of supernumerary innervation at the NMJ has been further demonstrated in a collection of studies that provided additional clues to the underlying mechanism. Chronic 


\section{Chapter 1}

inhibition of synaptic signaling through either irreversible blockage of AChR through bath application of the antagonist $\alpha$-bungarotoxin (Balice-Gordon and Lichtman, 1994) or through the overexpression of postsynaptic inwardly rectifying potassium channels that inhibit the generation of action potentials (Favero et al., 2009) could dramatically stall synaptic pruning. Alternatively, driving synchronous activation across all supernumerary inputs undergoing synaptic competition too stalls selective pruning and induces the maintenance of polyinnervation in muscle fibers (Colman et al., 1997; Busetto et al., 2000; Favero et al., 2010). Thus, a central requirement for the selection of appropriate inputs during competition is a measurable disparity in synaptic strength and spike timing among competing inputs and in fact an artificial enhancement in these disparities can hasten the removal of less active inputs (Thompson, 1983; Keller-Peck et al., 2001; Favero et al., 2012).

Several key molecules have been identified that have a strong molecular influence on competition in this system (Tintignac et al., 2015). These signaling molecules released from competing terminals can have dramatic effects on NMJ development by affecting the competitive vigor of afferent co-competitors and also through selective reinforcement of a single input. A molecular mechanism proposed in the late 1970s by Obrien et al. pointed at indirect competition between MNs along developing rat muscles (Obrien et al., 1978). In some elegant experiments, it was demonstrated that activation of MNs led to the release of not only ACh but also proteinase enzymes that, at sufficient concentrations along a developing muscle fiber, act to non-selectively disrupt synaptic adhesions (Obrien et al., 1978). Interestingly though, sustained levels of activity in a single afferent can counterbalance this effect via the delivery of new synaptic machinery through microRNA regulated retrieval of dense cored vesicles. This process bolsters the generation of new synaptic and adhesion sites under active terminals (Wei et al., 


\section{Chapter 1}

2013) and perhaps partially explains the effect of synaptic takeover that underpins the ultimate fate of monoinnervation at the motor endplate (Walsh and Lichtman, 2003). Agrin, a proteoglycan that serves as a proteinase inhibitor is also secreted from afferent terminals and may play a part in protecting active terminals from the secretion of proteinase enzymes (Obrien et al., 1978; Biroc et al., 1993). Moreover, agrin efficiently induces the clustering of AChR along the membrane of muscle fibers (Dulcert and Changeux, 1995). This is achieved through the interaction of agrin with the postsynaptic, and membrane-bound, muscle-specific tyrosine kinase, MuSK, which phosphorylates several targets including rapsyn to initiate postsynaptic density formation (PSD) (review by Sanes \& Lichtman, 1999). Thus, the agrin-mediated cascade may help to organize the random and prepatterned assortment of motile AChRs (described as randomly 'walking' receptors before association with PSDs) on the surface of early postnatal myotubes into dense clusters directly under active terminals (review by Sanes \& Lichtman, 2001).

\section{Climbing fiber innervation of Purkinje cells}

The peripheral, activity-dependent competition and pruning process required for the development of the NMJ has strong similarities to another well described monoinnervating central connection, the climbing fiber (CF) to Purkinje cell (PC) terminal (Lorenzetto et al., 2009). The cerebellar CF represents a powerful, excitatory input that undergoes similar stages of synaptic competition to the NMJ (Watanabe and Kano, 2011). This neural circuit was initially studied by Ramón y Cajal (Ramón y Cajal, 1911; de Castro et al., 2007). At birth in mice, CF axons generate as many 100 en passant boutons onto the somata of PCs, during what has been 


\section{Chapter 1}

termed the 'creeper stage'. Between P4-P7 and during what is termed the 'pericellular stage', only about $10 \%$ of these initial CF arbors remain and the soma is innervated by $\sim 4-5$ unique and larger nest-type inputs (Sugihara, 2005). The afferents are of moderate synaptic strength and evoke similarly sized postsynaptic currents during the short-lived pericellular stage (Hashimoto and Kano, 2003) as synaptic competition begins to resolve polyinnervation during final stages of development, termed the 'capuchon and dendritic stages', during the second postnatal week. The capuchon stage is hallmarked by the emergence of 2-3 inputs that increase in size and strength (Hashimoto and Kano, 2005). During this period of active competition, the remaining competitors also begin to shift position along the soma of the PC where the 'winning' input, that generates the largest postsynaptic current, translocates to the base of the apical dendrite of the PC. This input grows with and innervates the PC dendrite while the remaining smaller inputs are sequentially removed from the soma and pruned to produce a strong mono-innervating connection (Hashimoto et al., 2009). Similar to the NMJ, the process of selective refinement of excess CFs is dependent on disparate levels of activity between inputs. A dramatically sharp period between P15-16 mice appears to define the largest period of active NMDA-receptor mediated synaptic competition in this system wherein MK-801 administration antagonized NMDA receptors and inhibited removal of excess innervation (Kakizawa et al., 2000). Similarly, administration of harmaline to drive synchronous activation of competing CFs too has been shown to inhibit synapse elimination (Andjus et al., 2003).

The CF:PC system, like the NMJ, has been a useful model to identify molecular underpinnings of synapse removal. Here a cytoskeletal protein called Arc has been implicated in stability and maintenance of somatic CF inputs during development through selective endocytotic induction of AMPARs (Chowdhury et al., 2006). Arg3.1, the gene encoding Arc, is 


\section{Chapter 1}

upregulated following activation and $\mathrm{Ca}^{2+}$ entry through enriched P/Q-type channels in PCs (Mikuni et al., 2013). Once translated, Arc preferentially binds the calmodulin-dependent protein kinase II $\beta$ (CaMKII $\beta)$ that is enriched at recently activated synapses (Okuno et al., 2012). Repetitive $\mathrm{Ca}^{2+}$ influx produces a persistent association between $\mathrm{Ca}^{2+} /$ calmodulin and CaMKII $\beta$ that induces autophosphorylation of CamKII $\beta$ and prevents association with Arc, thereby stabilizing the most active synaptic sites (Yamauchi, 2005). This activity-mediated mechanism alters the balance of activity among competing inputs and can indirectly induce the selective maintenance of some and the removal of others.

\section{Retinal Ganglion Cell Innervation of the Dorsal Lateral Geniculate Nucleus}

A major projection of the mammalian retina, the dorsal lateral geniculate nucleus (dLGN), represents another key model system for understanding the process of selective circuit formation in the central nervous system. Here, retinal ganglion cells (RGCs) that have partially crossed the optic chiasm will arborize, innervate and terminate widely across midbrain visual centers including the superior colliculus (McLaughlin et al., 2003a) and importantly for this discussion within the dLGN (Guido, 2008). The majority (85-90\%) of afferents within the dLGN are of RGCs originating from either eye owing to the partial decussation of the optic nerve fibers across the optic chiasm, at least in mice (Guido, 2008). The resulting pattern of binocular innervation within the dLGN is comprised of two underlying forms of organization fundamental to visual processing; afferent projections from each eye form non-overlapping territories in the mature dLGN (Godement et al., 1984; Muir-Robinson et al., 2002) and critically, the spatial 


\section{Chapter 1}

nasal-temporal arrangement of the retina is topographically mirrored in the dorsal-ventral axis of the dLGN (Torborg and Feller, 2005).

Development of the dLGN can be divided into distinct phases that occur before animals begin processing much of the visible world. The first phase of development relies on chemotactic guidance wherein RGC axons must navigate to appropriate territories within the relay nuclei of the visual system. This is achieved after RGC axons have left the eye wherein neuroepithelial Sema5a expression generates a repulsive signal that concentrates axons tightly within the optic tract. Axon growth is promoted towards the ventral diencephalon via a graded expression of ephrin-A that is sensed by axonal membrane-bound Eph-A receptors (Oster et al., 2003; Flanagan, 2006). Ephrin-B2 is expressed by midline radial glia (Williams et al., 2003), and contralaterally projecting RGCs that do not express EphB1 will cross the optic chiasm, arriving at the dLGN by E16 in mice. Two days later the non-crossing ipsilateral projections arrive by E18 (Godement et al., 1984). Soon after, axons extensively arborize in response to molecular cues encountered during the immediate perinatal period until approximately P4 (Godement et al., 1984). Chemotaxic guidance to the thalamic dLGN produces an initially overlapping innervation pattern representing inputs from both eyes (Guido, 2008). A second phase of development involves the structural refinement of exuberant RGC axonal projections in order to produce the final arrangement of retinotopic organization (Penn et al., 1998). During the end of the first postnatal week, between P4-8, selective pruning begins to reduce the extent of exuberant connectivity e (Godement et al., 1984; Bickford et al., 2010). These remaining inputs are subsequently reinforced between P8-12 when at increase in vesicle release sites results in much larger (up to $\sim 50 \mathrm{X}$ ) synaptic currents (Chen and Regehr, 2000). Ultimately, following the onset of sensory experience (P12-14), the innervation pattern of the dLGN largely resembles that of 


\section{Chapter 1}

the adult such that most relay neurons are driven by monocular innervation from between one to three RGCs.

Roles for neural activity were revealed by early experiments reducing whole eye input by ocular removal or pharmacological treatment (e.g., continuous delivery of TTX or glutamate receptor antagonist; (Garraghty et al., 1988; Shatz and Stryker, 1988; Hahm et al., 1991)). The proposed necessity for sources of activity within developing circuits was further reinforced as contemporary researchers began identifying patterns of spontaneous activity wherein neighboring RGC projections fire nearly synchronous bursts of action potentials (APs), separated by minutes, that propagate in waves across the entire retina (Meister et al., 1991). These waves were further preserved across the dLGN (Mooney et al., 1996). Thus, the next step in understanding the development of the visual system required more strategic manipulation to probe for possible underlying Hebbian mechanisms, whereby such spatially relevant patterns of repetitive presynaptic firing may selectively favor the maintenance of more correlated inputs while uncorrelated activity may target others for selective removal in a fashion that followed Hebb's postulate (Hebb, 1949).

The important question to be answered was whether the pattern of spontaneous activity was crucial for the generation of eye-specific layering in the visual system. Initial attempts at unilateral pharmacologic manipulation of RGCs in developing ferrets targeted the generation of patterned spontaneous activity and produced significant interruption in ocular layering organization in the downstream dLGN (Penn et al., 1998; Stellwagen and Shatz, 2002). Importantly, more targeted genetic approaches that disrupt the generation of spatiotemporally correlated activity between neighboring RGC axons from the same eye (through genetic abolishment of $\beta 2$ subunit of the nicotinic acetylcholine receptor) too generated significant 


\section{Chapter 1}

alterations synaptic refinement without the necessity to account for surgical effects (Grubb et al., 2003; McLaughlin et al., 2003b). Thus, correlated and patterned RGC firing during the period of early postnatal development has been repeatedly found to represent a powerful requirement for selective refinement of initially exuberant RGC terminal fields necessary to generate topographically organized, eye specific segregation of inputs to the dLGN, essential for higherorder visual processing to enable the perception of binocular vision once relayed to the cortex (Torborg and Feller, 2005).

\section{Calyx of Held Innervation of the Medial Nucleus of the Trapezoid Body}

The auditory system too serves as an important model for studying the formation of a circuit necessary for the computation of aural cues such as sound localization (Grothe et al., 2010). The primary aim of my dissertation research has been to describe the structural development of the calyx of Held $(\mathrm{CH})$ nerve terminal and elucidate the competitive interactions that occur during the formation of this structure.

The $\mathrm{CH}$ was first described in seminal sparse Golgi staining experiments performed just prior to the 20th Century by Hans Held (Held, 1893). Soon after, Ramón y Cajal, H. WaldeyerHartz and others described examples of neuronal structures with observable pre and post-contact delineations between neurons in support of the Neuron Doctrine (López-Muñoz et al., 2006). In more recent decades, technological advances in cellular and molecular experimentation paired with the exaggerated morphology of the $\mathrm{CH}$ permit the direct manipulation and observation of the function and plasticity of a nerve terminal with hundreds of discrete synapses that provides fast, robust and high-fidelity stimuli to single principal cell targets in the medial nucleus of the 


\section{Chapter 1}

trapezoid body (MNTB) within the auditory brainstem (Forsythe, 1994; Meyer et al., 2001).

Moreover, the $\mathrm{CH}$ :MNTB connection exhibits coordinated pre and postsynaptic maturation (Hoffpauir et al., 2010) and undergoes presynaptic competition during development with two distinct phases of presynaptic strengthening and pruning mirroring synaptic development of the NMJ, CF:PC and RGC:dLGN (Hoffpauir et al., 2006; Holcomb et al., 2013). Thus, the development of the $\mathrm{CH}: \mathrm{MNTB}$ connection represents another strong model for synaptic development with a demonstrable resolution to monoinnervation by maturity that will remain the central focus of this dissertation. The remainder of this document will largely focus on describing the sequence of events pertinent to the formation and maturation of the $\mathrm{CH}$ nerve terminal that innervates the principal cell of the MNTB.

\section{Development of the Calyx of Held}

Charles Stockard first proposed a general concept of a 'sensitive period' to the field of developmental neurobiology in the 1920 s in order to describe the confluence of permissible cues that exist during a limited period in development and allow for massive plasticity within circuits in the brain (Stockard, 1921). This idea was later reinforced in seminal animal studies utilizing reversible monocular deprivation studies, first in cats (Hubel and Wiesel, 1965; Wiesel and Hubel, 1965), and later in primates (Hubel et al., 1977). Further, this general concept of a sensitive period has proven useful in describing the development of other sensory systems as well, including the auditory system (Kandler et al., 2009). In these cases, development is controlled with tight spatial and temporal regulation wherein the confluence of genetic regulation 


\section{Chapter 1}

(Kang et al., 2013; Miller et al., 2014), onsets and changes in the pattern or onsets of synaptic activity (Blankenship and Feller, 2010) and the arrival of specific subsets of cells (such as radial glia, for example, that produce new neurons through asymmetric cell division (Noctor et al., 2004)) can all play vital roles in the genesis and reorganization of neural tissues during early development.

In rodents, the $\mathrm{CH}$ forms soon after birth and undergoes dramatic morphological transformations during a brief postnatal period in order to form a terminal capable of fast and reliable transmission of auditory information that percolates through the auditory system within 10 days of birth in mice, comparable to the stages of development in a human fetus occurring after about 28 weeks of gestation (Lecanuet and Schaal, 2002). The terminal's large size is reflective of its proportionally large number of active zones, which across all sites are capable of releasing more than a hundred synaptic vesicles during a single depolarizing event (Borst and Sakmann, 1996). Such robust signal transmission guarantees a single action potential is capable of generating a suprathreshold depolarization in the postsynaptic principal neuron to critically preserve timing information necessary for auditory processing (Trussell, 1999). Another important feature of the $\mathrm{CH}$ that is shared with much of the mature auditory system is a strong trajectory bias towards precise tonotopic organization. Tonotopy within brainstem auditory nuclei is largely established during early perinatal development, before animals are exposed to environmental auditory stimuli, suggesting development here arises from a sensory-independent set of mechanisms (Friauf and Lohmann, 1999; Kandler et al., 2009; Clause et al., 2014). However, much like in the development of the NMJ, CF:PC and RGC:dLGN connections, synaptic competition and selective refinement of supernumerary connections within the developing MNTB occurs during a brief postnatal period to establish precise connectivity 


\section{Chapter 1}

(Holcomb et al., 2013). A comprehensive review of the role and development of the CH:MNTB within the auditory system will be provided in the following sections.

\section{Role of the Calyx of Held in Auditory Processing}

The spiral ganglia neurons that form the auditory nerve relay peripheral auditory information from the inner ear to the brain via innervation of the cochlear nucleus $(\mathrm{CN})$ in a way that preserves the tonotopic arrangement of the cochlea (Kandler et al., 2009; Muniak et al., 2013). Importantly too, this low to high frequency-dependent organization is further maintained throughout the ascending brainstem circuit by the onset of hearing in mammalian species (Kandler et al., 2009). The CN can be subdivided into the two primary nuclei, the dorsal and ventral cochlear nuclei (DCN and $\mathrm{VCN}$ ). Within the $\mathrm{VCN}$, a variety of neuronal populations exist with unique projections important for auditory processing including the so-called bushy cells. Of the two subtypes of bushy cells, the first are spherical bushy cells that provide excitatory ipsilateral input to neurons of the medial and lateral superior olive (MSO;LSO) as well as an additional projection to the contralateral MSO (Smith et al., 1993); the second class are referred to as globular bushy cells (GBC) which send a primary efferent projection to the contralateral MNTB giving rise to a $\mathrm{CH}$ but too less consistently project collateral innervation to both the ipsilateral superior olivary complex (SOC) targets including the periolivary nuclei, LSO, and lateral nucleus of the trapezoid body (LNTB) and contralateral targets such as periolivary nuclei including the MNTB, ventral nuclei of the trapezoid body and ventral nucleus of the lateral lemniscus (VNLL)(Spirou et al., 1990; Smith et al., 1991; Cant and Benson, 2003)(Fig 2). 


\section{Chapter 1}

Moreover, the arrangement of afferents and targets throughout the auditory brainstem preserve frequency-specific coding to maintain tonotopic organization.

Relevant for sound localization, the SOC is the first site to receive a convergence of binaural signaling. The shape and orientation of the two ears relative to each other produce submillisecond differences in the arrival time of bilateral signals. This allows the computation of differences in intensities, aural levels and arrival times of auditory cues with incredibly fast and reliable transmission $(<300 \mathrm{~Hz}$ ) (Taschenberger and von Gersdorff, 2000; Tollin, 2003; Grothe et al., 2010; Borst and Soria van Hoeve, 2012). Thus, by receiving tonotopically organized afferents from both ipsilateral and contralateral $\mathrm{CN}$ projections, the nuclei of the SOC are responsible for the initial integration and relay of highly organized bilateral auditory cues (Kandler et al., 2009).

\section{Initial Genesis of the Auditory Brainstem}

Soon after the closure of the neural tube during embryonic development, the rhombencephalon can be ultimately subdivided into 12 subregions, called rhombomeres (r). Each rhombomere has been shown to produce unique lineages of cells owing to differential expression of unique sets of transcription factors permitting the identification of discrete rhombomeres as early as E11-14 in mice, or approximately E29 in humans (Farago et al., 2006; O'Rahilly and Müller, 2007; Di Bonito et al., 2013). Importantly for the basis of this report, we will focus on two important auditory nuclei that develop specifically from this collection of rhombomeres: the ventral cochlear nucleus (VCN), which contains globular bushy cells and which arises from the caudal rhombic lip of r2-4 (Farago et al., 2006) and the MNTB, where the CH forms onto its 


\section{Chapter 1}

principal cells, and that likely arises from the proliferative dorsal rhombic lip of rhombomeres $\mathrm{r} 4$ and r5 between E9-14 in mice (Maricich et al., 2009; Di Bonito et al., 2013; Marrs et al., 2013).

\section{Establishment of GBC-MNTB Connections}

GBCs undergo neurogenesis beginning as early as E10 in mice and soon after project axons out of the VCN (Pierce, 1967). These axons arrive at the midline as early as E13.5 in mice (Howell et al., 2007) and subsequently cross into the presumptive contralateral SOC by E16 (Kandler and Friauf, 1993; Howell et al., 2007). During a similar gestational period between E16.5-E17.5, differentiating principal neurons within an amalgam of SOC cell groups in the ventrolateral brainstem begin to migrate medially across the brainstem to form a discernable MNTB that meets the arriving GBC axons (Hoffpauir et al., 2010; Marrs et al., 2013). Similar to commissural spinal neurons, GBC neurons rely on the expression of key guidance signaling to appropriately reach their newly arriving contralateral targets. In brief, GBC axons express a membrane bound receptor, DCC, that steers the outgrowth of axons along the ventral brainstem towards a gradient of netrin-1 secreted from and concentrated near cells at the midline (Nakamura and Cramer, 2011). Loss of either DCC or netrin 1 results in an inability for GBC axons to appropriately reach the midline (Howell et al., 2007). Once axons have successfully navigated the midline however, a molecular switch occurs whereby DCC loses its sensitivity to netrin-1 and axons are further promoted in a trajectory away from the midline. Secreted Slit-1 secreted acts through Robo-1 and Robo-2 receptor mediated repulsion to effect axon guidance. Similar to knockout models of DCC (Fazeli et al., 1997) or netrin-1 (Serafini et al., 1996), conditional abolishment of Robo-3 produced errors in appropriate GBC axonal targeting and 


\section{Chapter 1}

resulted in aberrant ipsilateral connectivity via a failure to cross the midline (Michalski et al., 2013).

Microscopy studies in embryonic rodent tissues have demonstrated newly arriving GBC axons at E15 next experience a brief waiting period within the MNTB of about two days before axonal contacts onto principal neurons are established just before birth (Kandler and Friauf, 1993). Further confirming the existence of a waiting period prior to innervation, slice experiments that utilized in vitro preparations that maintained axonal projections from the cochlea to the brainstem demonstrated bushy cell axons could not elicit responses in principal neurons until E17.5 (Hoffpauir et al., 2010; Marrs and Spirou, 2012), although bushy cells themselves could be driven by spiral ganglion neuron (SGN) input as early as E15 (Marrs and Spirou, 2012). Spontaneous activity through this connection has been measured during the immediate perinatal period between E17 and P2 in the MNTB despite little if any growth occurring during this period (Hoffpauir et al., 2010). The initial contacts made by calyciferous axons onto between 5-20 discrete principal neurons per axon each remain small into postnatal ages as no large terminals have been detected earlier than P3 in mice (Hoffpauir et al., 2010; Holcomb et al., 2013).

\section{Formation of a Large Terminal}

The phenomena that trigger the explosive growth of the $\mathrm{CH}$ have only partially been elucidated. A genome-wide microarray analysis comparing gene expression in the MNTB and LSO identified the bone morphogenic proteins (BMPs) as enriched in the MNTB during calyx growth as well. Genetic deletion of BMP receptors using an Engrailed-2 (Egr2) cre line led to a 


\section{Chapter 1}

disruption in growth and competition of the $\mathrm{CH}$ as inputs failed to progress past the stage of protocalyceal poly-innervation (Xiao et al., 2013). Loss of BMPR1a/1b genes in this context may have impaired $\mathrm{CH}$ growth in a due to disruption in downstream signaling. First, loss in typical levels of BMPR1a and BMPR1b-mediated phosphorylation of the transcription factor SMAD likely alters the capacity for SMAD to modifying downstream gene regulation (Miyazono et al., 2010). Alternatively, loss of BMP association with BMPR1a/1b may induce a deficit in SMADindependent regulation of axon dynamics that further disrupts $\mathrm{CH}$ formation (Zhong and Zou, 2014). Wildtype mice express the serine/threonine-protein kinases SAD-A and SAD-B both pre and postsynaptically in the MNTB. SAD-A/Sad-B double knock-out mice show significant deficits in calyx growth owing to an inability to regulate cytoskeletal phosphorylation (AlvaradoKristensson et al., 2009; Lilley et al., 2014). Aiming for another possible molecular candidate, knock-out experiments targeting neural recognition molecule 2 (NB2), which plays a role in synapse stability and cell-cell adhesion, led to a minor disruption in calyx growth and stability (Toyoshima et al., 2009). Unfortunately, none of these perturbations entirely prevent the formation of a presynaptic terminal in this system or fully explain the rapid formation of the characteristically large $\mathrm{CH}$.

At $\mathrm{P} 3$ in rodents, GBC axonal termini in the murine neonatal MNTB begin to rapidly transition into spoon-shaped terminals called the protocalyx (Morest, 1968a; Kandler and Friauf, 1993; Holcomb et al., 2013)(Fig 3). Robust expansion of the protocalyx coincides with the onset

of patterned, experience-independent spontaneous activity that begins to percolate throughout the auditory system soon after birth (Beutner and Moser, 2001; Tritsch and Bergles, 2010). The spontaneous bursting activity pattern typical of the developing postnatal auditory system originates within the transient Kölliker's organ of the immature cochlea. There, a mass of 


\section{Chapter 1}

columnar supporting cells nearby to inner hair cells (IHCs) secrete ATP locally within the organ as IHCs concurrently become responsive to purinergic signaling. This spontaneous release and buildup of ATP readily depolarizes maturing IHCs and triggers bursts of action potentials that subsequently stimulate the dendrites of Type I spiral ganglion neurons. Moreover, irregular changes in local ATP concentrations across the developing cochlea produce differences in the pattern of activity among IHCs; neighboring IHCs more synchronously depolarize relative to more distant pairs of IHCs. Moreover, these differences in local firing among IHCs across the cochlea further produce differences in firing patterns among first order spiral ganglion neurons (Tritsch et al., 2007; Tritsch and Bergles, 2010).

In order to investigate the necessity for spontaneous activity in the development of the auditory system, manipulations that experimentally alter the temporal fine structure of spontaneous bursting activity in the developing cochlea have been shown to measurably affect the tonotopic organization of downstream nuclei in the auditory system (Clause et al., 2014). Thus, it is tempting to speculate that the onset of spontaneous activity found to temporally coincide with the expansion of the terminal may serve as a trigger for growth and the pattern of activity may be useful for the selection of appropriate connections such as occurs in the visual system (Wong et al., 1993; Ackman et al., 2012).

\section{Morphology of the Protocalyx}

Morphological surveys of developing protocalyces have revealed insights into the potential mechanisms of large terminal formation in this system. For example, much like the $\mathrm{CF}: \mathrm{PC}$ system wherein the dominant input takes a position at the base of the apical dendrite 


\section{Chapter 1}

(Hashimoto et al., 2009; Watanabe and Kano, 2011), the development of the CH may be contingent on the location of initial innervation during its period of synaptic competition. At early postnatal ages, before the onset of hearing in mice (P3-9), MNTB principal cells exhibit a polarized morphology with an eccentrically located nucleus that is biased towards one end of the cell, defined as the nuclear pole. Moreover, in the first survey of $\mathrm{CH}$ development using serial electron microscopy it was noted the emerging protocalyces were excluded from growth over this nuclear pole (Hoffpauir et al., 2006). This observation was later confirmed in a more systematic analysis of $\mathrm{CH}$ formation across 92 cells (11 at P2, 29 at P3, 17 at P4, 29 at P6, and 11 at P9) that further demonstrated the prevalence of nuclear eccentricity in the developing MNTB. Further, the study reported a significant bias favoring growth of the largest protocalyceal terminal from multiple large competitions occurs on the non-nuclear pole of MNTB principal cells (Holcomb, 2018). This type of permissive growth towards a preferred somatic region along a postsynaptic soma closely resembles the established mechanism of selective reinforcement of competing terminals described in the CF development (Hashimoto et al., 2009; Watanabe and Kano, 2011).

As the protocalyx expands from initial en passant axonal projections into enlarged, spoon-shaped terminals, it characteristically produces abundant cytoplasmic extensions, defined as thin collateral stalks, in a process that parallels axonal arborization (Fig 3). These collateral structures radiate dozens of microns away from the immediate innervation territory of the developing terminal (Kandler and Friauf, 1993). Akin to developing axons and dendrites, calyceal collaterals can be tipped with growth cones and often support numerous branches as well. Importantly, these collateral features of the emerging $\mathrm{CH}$ persist as a common feature only during the neonatal period of calyceal development (Morest, 1968a; Kandler and Friauf, 1993). 


\section{Chapter 1}

Although the targets of calyceal collaterals are unknown, $\mathrm{Ca}^{2+}$ imaging in vivo demonstrated the collaterals generate presumptive synaptic signals, thus increasing the extent of presynaptic innervation territory beyond a single terminal target during early development. However, much is still unknown about these structures and their function during development and the subsequent chapter will aim to investigate their role during development wherein their function may serve to establish or maintain innervation territory during a chaotic period of rapid growth and synaptic competition.

\section{Transient Period of Synaptic Competition}

As described previously, developing axons in many parts of the nervous system produce exuberant branches within targeted territories. In order to achieve synaptic specificity reflective of more mature connectomes, an activity-dependent process of synaptic competition favors the stabilization of a subset of inputs and the removal of others (Hua and Smith, 2004; Ooyen, 2005; Schuldiner and Yaron, 2014). It has remained a fairly contentious issue in the literature as to whether the development of the $\mathrm{CH}$ can be described as proceeding according to this canonical program of early exuberance followed by synaptic competition or if, in fact, the connections form with extreme tonotopic precision owing to genetic or molecular guidance (Rodríguez-

Contreras et al., 2006; Kandler et al., 2009). In part, this may be due to the rapid establishment of dominant monosynaptic innervation in this system that emerges between P4-6 (Hoffpauir et al., 2006; Holcomb et al., 2013).

The number of large, competing inputs innervating principal neurons in the MNTB has been assessed through minimal stimulation paradigms in which single inputs are recruited 


\section{Chapter 1}

through step-wise increases in external stimulation (Hoffpauir et al., 2010; Xiao et al., 2013). Initial physiologic studies revealed multiple large inputs, but at relatively low percentages (4 in 29 cells at (P4) (Hoffpauir et al., 2006) and 6 in 101 cells nearer the onset of sensitivity to airborne sounds (P7-12)(Bergsman et al., 2004). Systematic reconstructions from volume electron microscopy images has revealed a higher proportion of competing inputs (9/16 at P3; 6/18 at P4; 10/42 at P6) (Holcomb et al., 2013). In part this discrepancy could be explained in a few key ways. The generation of an acute brain slice for in vitro study inherently reduces the number of maintained synaptic connections through physical severance of many axons as well as potentially damaging any cells accessible to whole-cell patching in superficial layers near the cut plane. Alternatively, the protocalyx has been defined as large when its area of direct apposition is greater than $35 \mu \mathrm{m}^{2}$, which, relative to the mature $\mathrm{CH}$, is still considered a relatively small input that may have been overlooked in electrophysiologic surveys of large terminals (Holcomb et al., 2013). Similar to the NMJ, the progressive formation of the large $\mathrm{CH}$ nerve terminal passes through two distinct phases of strengthening and pruning to a single, large nerve terminal. However, these events in the MNTB occur more quickly (24-48 hours) relative to other classically described systems (Holcomb et al., 2013).

\section{Early Stage Strengthening and Pruning}

The first stage of calyceal strengthening and pruning occurs during the perinatal period from E17 until P3. Following arborization of newly arriving GBC axons within the MNTB, each principal neuron becomes innervated by $10-20+$ small bouton-type or en passant GBC axonal endings (Holcomb et al., 2013). From among these superfluous connections, at most two or three 


\section{Chapter 1}

inputs per neuron are strengthened and permitted to grow large $\left(>35 \mu \mathrm{m}^{2}\right)$ between $\mathrm{P} 3-4$ (Kandler and Friauf, 1993; Hoffpauir et al., 2006). In this context, apposed surface area is used as a proxy metric for synaptic strength as studies have shown the number of active zones increases with age and in proportion with the size of the terminal (Taschenberger et al., 2002; Holcomb et al., 2013). During this early period wherein inputs are still small $\left(<35 \mu \mathrm{m}^{2}\right)$, single inputs to the principal neuron are only capable of eliciting proportionally small postsynaptic currents, suggesting release from only a small number of active zones per terminal (Hoffpauir et al., 2006). However, in order to maintain high frequency spike rates, the immature terminals employ multiple $\mathrm{Ca}^{2+}$ clearing mechanisms including ion channel exchangers and specialized mitochondria anchored to the presynaptic membrane by filamentous supports to form ancillary conglomerate structures referred to as mitochondria-associated adherens complex (or MACs) located adjacent to a presynaptic membrane nearest the readily releasable pool of synaptic vesicles to increase the efficiency of neurotransmitter release via efficient $\mathrm{Ca}^{2+}$ sensing and local ATP production (Rowland et al., 2000; Kim et al., 2005). These adaptations allow competing protocalyces to quickly become even more potent inputs and hence stronger competitors.

\section{Late-Stage Strengthening and Pruning}

During a second distinct phase of strengthening and pruning, the subset of remaining large protocalyces $\left(2-3 ;>35 \mu \mathrm{m}^{2}\right)$ compete to establish a dominant innervation territory along each principal cell. Importantly, the mode of competition in this context is indirect as terminals form largely non-overlapping territories on single postsynaptic principal neurons (Holcomb et al., 2013), although significant changes in the postsynaptic cell described in a subsequent section 


\section{Chapter 1}

may mediate a postsynaptic mechanism with which the competitive vigor of competing inputs may be assessed. This second phase of competition in this system lasts only a brief period $(<2-3$ days) and involves both the selective removal of unneeded GBC innervation and the rapid emergence of a single, large and spoon-shaped protocalyx on most principal cells, at an accelerated growth rate that may exceed $200 \mu \mathrm{m}^{2}$ per day at its peak during this period (P6 75\%; P9 88\% monoinnervated) (Bergsman et al., 2004; Holcomb et al., 2013). Interestingly though, although the developing MNTB has been imaged in vivo (Rodríguez-Contreras et al., 2008), the selective withdrawal of excess large innervation has yet to be directly observed and may in fact outpace even the rapid expansion of a protocalyx.

\section{Maturation and Fenestration of the $\mathrm{CH}$}

Sound localization can only be achieved with precise synaptic processing across the auditory system and the $\mathrm{CH}$ :MNTB connection in this regard serves as an exemplary example of specialized adaptation. Following the opening of the ear canal and the onset of sensitivity to airborne sound (P10-12), the $\mathrm{CH}$ has perhaps achieved its greatest size. Across this large area of apposition, hundreds or thousands, depending on species, of discrete adherens junctions and presynaptic active zones provide increased mechanical stability and robust excitation (Morest, 1968a; Kandler and Friauf, 1993; Rowland et al., 2000; Sätzler et al., 2002; Hoffpauir et al., 2006). In order to maximize the temporal fidelity of this terminal, there are additional adaptations that must be implemented through complex morphologic and physiologic changes. After P10, protocalyces both structurally consolidate through the retraction of thin collaterals stalks and transform from a "hairy" spoon-like shape into the stereotypically smooth, fenestrated 


\section{Chapter 1}

or "finger-like" arrangement of axo-somatic contacts that encompass a substantial proportion (as much as $50 \%$ ) of the postsynaptic soma. This new morphology allows for more efficient clearance of glutamate from the synaptic cleft during high frequency transmission (Ford et al., 2009). Despite the morphology of the $\mathrm{CH}$ permitting synchronous release of glutamate from throughout the terminal, the mature $\mathrm{CH}$ also exhibits a characteristically shorter duration AP $(\sim 25 \%)$ that allows little opportunity for presynaptic calcium influx (Taschenberger and von Gersdorff, 2000; Yang and Wang, 2006). In turn, this is reflected in a relatively low release probability at single active zones throughout the $\mathrm{CH}$. Thus, to maintain synaptic activity during high frequency stimulation with high fidelity necessary for acute auditory processing, it is necessary for $\mathrm{Ca}^{2+}$ to become more tightly regulated to increase the probability of single release (Wang et al., 2008; Kochubey et al., 2009). This is achieved as immature protocalyces shift from a reliance on $\omega \mathrm{CgTx}$ GVIA-sensitive N-type and $\omega$ Aga-IVA-sensitive P/Q type voltage gated $\mathrm{Ca}^{2+}$ channels to predominantly a P/Q-type current driven by the subunit Cav2.1 (Iwasaki and Takahashi, 1998) and Kv3 channels (Wang et al., 1998; Elezgarai et al., 2003) which cluster tightly with active zones nearby the readily releasable pool of vesicles (Iwasaki and Takahashi, 1998; Fedchyshyn and Wang, 2005; Wang et al., 2008; Chen et al., 2015). Together, these final presynaptic adaptations function to accelerate the repolarization of the membrane, shorten AP duration and increase synaptic fidelity to aid in the transformation of the $\mathrm{CH}$ into a fast and reliable relay for mature sensory processing.

\section{Maturation of the Postsynaptic Principal Cell}




\section{Chapter 1}

The maturation of the postsynaptic principal cell mirrors the developmental changes seen presynaptically. Immature principal cells in the MNTB at birth have a high input resistance $(>1 \mathrm{G} \Omega)$ and a relatively depolarized resting membrane potential $(-55 \pm 7 \mathrm{mV})$ making them extremely excitable and capable of generating action potentials as early as E17 in response to minimal synaptic current downstream of spontaneous neurotransmitter release $(>30 \mathrm{pA})$ (Hoffpauir et al., 2010; Rusu and Borst, 2011). However, soon after birth (P2) the pattern of spontaneous activity measurable in this system dramatically shifts towards bursts of bursts (Tritsch and Bergles, 2010). Such repetitive activation makes these cells susceptible to tonic excitation that may lead to increased $\mathrm{Ca}^{2+}$ concentrations, especially through upregulated GluA2deficient $\mathrm{Ca}^{2+}$-permeable AMPA receptors at early postnatal ages (Lujan et al., 2019), known to directly induce gene expression in the developing nervous system (Flavell and Greenberg, 2008). Once protocalyces emerge between P2-3, these glutamatergic terminals begin to drive EPSCs in principal neurons characteristically appoaching $-1 \mathrm{nA}$. The $\mathrm{CHs}$ that emerge soon after, however, are capable of driving currents exceeding -6 nA by P4 (Futai et al., 2001; Hoffpauir et al., 2006). This rapid change in input strength means principal neurons too must rapidly evolve more mature firing properties in concert with the rapid growth of the presynaptic protocalyx. Postsynaptic cells during this period transition from tonic to phasic response patterns (Hoffpauir et al., 2010). The principal cells, in part, progressively gain this ability by P6 through a reduction in their intrinsic excitability as the resting membrane potential increases to $-72 \mathrm{mV}$, the input resistance drops roughly 5-fold to $\sim 200 \mathrm{M} \Omega$ and the expression of low voltage-gated potassium channels (Kv1.1 and Kv1.2) increases which together act to inhibit tonic postsynaptic responses and tune MNTB neurons towards single spikes (Brew and Forsythe, 1995; Dodson et al., 2002; Hoffpauir et al., 2010). Moreover, these postsynaptic changes may provide a means for each 


\section{Chapter 1}

principal neuron to tune its firing to the competing input that drives the strongest and most synchronous activity, similarly to the development of the NMJ (Favero et al., 2007).

The changes in biophysical properties of principal cells during this early postnatal period also mirror events occurring in other auditory nuclei including a loss of dendritic arbors in the presence of spontaneous bursts of activity (Rietzel and Friauf, 1998; Franzen et al., 2014). The cell body also significantly increases in volume, perhaps through partial resorption and membrane recycling (Hermida et al., 2010; Hoffpauir et al., 2010). The intrinsic charging capacity of the cell is clearly altered by this structural increase in cell size as membrane capacitance and resistance decreases dramatically whilst current threshold is significantly increased (Hoffpauir et al., 2010; Rusu and Borst, 2011). Undeniably, these changes in the intrinsic biophysical properties of the postsynaptic cells collectively raise the thresholds to favor only the strongest presynaptic inputs and may bely a functional pressure necessary for the selection of a single dominant input.

The principal cells of the MNTB also undergo glutamate receptor subunit switching to alter firing patterns. The early neonatal principal neuron initially relies on the expression of AMPAR subunits GluR1/2 but is largely dominated by NMDARs as well which together provide slow decay properties in response to immature EPSCs (Caicedo and Eybalin, 1999; Taschenberger and von Gersdorff, 2000; Joshi et al., 2004). Over the course of the first week of life, the expression of AMPAR begin to switch as GluR1 expression decreases with age (Hermida et al., 2006). Moreover, the dramatic increase in synaptic strength and firing rate experienced between P3 and the onset of sensory experience in the MNTB triggers an increased expression of GluR3/4 (Caicedo and Eybalin, 1999; Joshi et al., 2004) and a sharp reduction in NMDAR along the postsynaptic membrane (Caicedo and Eybalin, 1999; Joshi and Wang, 2002). 


\section{Chapter 1}

These adaptations provide substantially faster AMPAR-EPSC kinetics required for this synapse to preserve timing information relevant in auditory cues (Trussell, 1999) and collectively tune the maturation of the MNTB principal neuron in concert with the development of its largest presynaptic input (Hoffpauir et al., 2010).

\section{Aim of the study}

The $\mathrm{CH}$ is the largest terminal in the mammalian brain and has been shown to develop very rapidly. However, to date, very few studies have tried to elucidate the dynamics that define the development of this system in terms competitive growth, directly or indirectly, from among immature protocalyceal terminals. From the establishment of a broad GBC axonal innervation territories in the MNTB, to the emergence and motility of elaborate secondary/terminal arborization and the functional strengthening of maturing $\mathrm{CHs}$, the goal has become to describe these processes from a more targeted perspective of synaptic competition during the first weeks of life. Here we study the fine changes in $\mathrm{CH}$ ultrastructure during early postnatal development, assess the interaction of competing $\mathrm{CHs}$, and chart the emergence of a rapidly maturing, functionally fast and high fidelity (faithfully phase locking up to $1 \mathrm{kHz}$ ) central synapse that is paramount to encoding timing information with respect to binaural auditory cues using advanced forms of high-resolution microscopy to chart the formation of the calyx of Held. 


\section{Chapter 2: Rapid Terminal Dynamics Within Target Territories During Neural Circuit Formation}

Dakota Jackson $^{1,2}$, John Heddleston ${ }^{3}$, Michael Morehead ${ }^{1,2}$, Teng-Leong Chew ${ }^{3}$, Stansilav Pidhorskyi1 $^{1,2}$, Paul Holcomb ${ }^{1,2}$, Shobhana Sivaramakrishnan ${ }^{1,2}$, Samuel Young ${ }^{4}$, Shilajeet Ray ${ }^{1,2}$, Thomas Deerinck ${ }^{5}$, Mark Ellisman ${ }^{5}$, and George Spirou ${ }^{6}$

${ }^{1}$ Rockefeller Neuroscience Institute, ${ }^{2}$ Sensory Neuroscience Research Center, WVU School of Medicine, Morgantown, West Virginia 26506

${ }^{3}$ Advanced Imaging Core, Janelia Research Campus, Ashburn, Virginia 20147

${ }^{4}$ Research Group Molecular Mechanisms of Synaptic Function, Max Planck Florida Institute for Neuroscience, Jupiter, Florida 33458

${ }^{5}$ National Center for Microscopy and Imaging Research, University of California, San Diego, La Jolla, California 92093

${ }^{6}$ Department of Medical Engineering, University of South Florida, Tampa, Florida 33620 Introduction: 


\section{Chapter 2}

The central nervous system is the most complex of organs and self-assembles its basic organization at extremely high rates. In mice, for example, about 100 million neurons are born during an eleven-day period between formation of the neural tube at embryonic day (E) 9 and birth. During this time frame the nervous system expands to a volume of $90 \mathrm{~mm}^{3}$ (De Felipe et al., 1997), and forms interconnections in neocortex at a density of about a trillion synapses per $\mathrm{cm}^{3}$ (Drachman, 2005). Soon after neurogenesis, growth-cone led axons begin extending toward targets that can be tens of micrometers to centimeters distant from their cell body, and must do so rapidly, at velocities estimated at up to $\sim 3 \mu \mathrm{m} / \mathrm{min}$ in many parts of the nervous system (Chien et al., 1993; Halloran and Kalil, 1994; Witte et al., 1996; Phan et al., 2010). Within terminal territories however, axon extension rates generally decline as axons begin to densely arborize and form immature synaptic contacts (Harris et al., 1987; Halloran and Kalil, 1994; O'Rourke et al., 1994; Rodríguez-Contreras et al., 2008). In monitoring this process directly, tissue stability, photobleaching and phototoxicity have often limited sample rate and constrained temporal resolution of time lapse experiments (Chien et al., 1993; Halloran and Kalil, 1994; Witte et al., 1996; Schlosshauer et al., 2003; Phan et al., 2010; Özel et al., 2015). We questioned, given the dramatic $\mathrm{CH}$ growth rates estimated in a previous investigation (Holcomb et al., 2013), whether $\mathrm{CH}$ dynamics would occur more quickly than has been previously reported in other systems.

More recently light sheet microscopy (LSM), which achieves volume acquisition at high sample rates with minimal tissue damage, has provided an imaging platform ideally suited to visualize morphologic changes within complex, 3D tissue geometry (Keller and Stelzer, 2008; Ahrens et al., 2013; Tomer et al., 2015). We have developed procedures to image brain slices using lattice light sheet (LLS) microscopy, which offers high temporal and spatial (near isotropic at the diffraction limit) resolution between image planes on scales useful for subcellular imaging 


\section{Chapter 2}

$(\sim 1 \mathrm{~Hz},<1 \mu \mathrm{m})$, higher signal-to-noise and substantially reduced phototoxicity and bleaching through delivery of thin optical sheets $(\sim 100 \mathrm{~nm})$ at low laser powers (Planchon et al., 2011;

Chen et al., 2014; Heddleston and Chew, 2016).

Topography in neural connections from cell group to cell group is a fundamental feature of brain organization, underpins neural computation, and has been explored in numerous studies over the past $100+$ years. Initially demonstrated and most easily studied in sensory systems, single neurons initially project broadly and then restrict projection territory, often in an experiential context. Mechanisms of synaptic competition are offered to explain these phenomena, but little explored is whether axon collaterals interact in space, away from the synaptic surface. We explored collateral process dynamics and their spatial interactions during formation of the calyx of Held $(\mathrm{CH})$ in the auditory brainstem, which exhibits an extreme and easily quantified version of topography, called tonotopy, via mono-innervation of its target in the medial nucleus of the trapezoid body (MNTB). The $\mathrm{CH}$ offers advantages as a developmental model (Kandler and Friauf, 1993; Hoffpauir et al., 2006, 2010; Crins et al., 2011; Holcomb et al., 2013; Xiao et al., 2013), because it passes through largely stereotyped phases of synapse formation including competition and pruning en route to mono-innervation similar to, but more quickly than (within 48 hours) other well-studied systems such as the neuromuscular junction (Sanes and Lichtman, 1999; Tapia et al., 2012) or the climbing fiber innervation of Purkinje cells within the cerebellum (Hashimoto et al., 2009; Watanabe and Kano, 2011). CH axons resolve to mono-innervation of $2 / 3$ of postsynaptic targets over a restricted 48 hour period (postnatal days (P)2-4) and increase in contact area at a rate of up to $200 \mu \mathrm{m}^{2} /$ day (Rodríguez-Contreras et al., 2006; Holcomb et al., 2013). Golgi and cell labeling studies first noted the existence of thin collateral processes of variable length, often tipped with growth cones, that extend from 


\section{Chapter 2}

immature terminals but are fewer and shorter by maturity (Morest, 1968a; Kandler and Friauf, 1993; Rodríguez-Contreras et al., 2008). An unspecified number of CHs innervate neighboring cells via collaterals, so these have potential to form permanent synaptic connections. In a tour de force, an in vivo two photon (2P) preparation was developed that revealed motility of $\mathrm{CH}$ collaterals. Notably, evidence from $\mathrm{Ca}^{2+}$ transients observed directly within these collaterals suggests a single calyx may functionally innervate multiple cells within the neuropil (RodríguezContreras et al., 2008).

These observations imply important roles for collaterals as: potential seekers of additional innervation targets, defenders of established innervation from other CHs, and/or vehicles for selective communication organizing frequency tuning across this auditory nucleus. To address these issues, we combined our unique volume EM series collected across developmental age with novel application of LLS microscopy. From these structural data, we defined collaterals types based on length and whether they were growth-cone or filopodialtipped. Long collaterals exhibited highly dynamic motility, and extended and retracted at higher rates than previously reported (Bray and Chapman, 1985; Halloran and Kalil, 1994; Bellar et al., 2013; Özel et al., 2015; Stanganello et al., 2015). Contact between collaterals was common as all CHs projected radiating collaterals densely throughout the MNTB neuroil. Furthermore, we show that a small population of postsynaptic neurons remain dually innervated into adulthood, and that occasionally these secondary terminals arise from collaterals extending from nearby CHs.

\section{Results:}




\section{Chapter 2}

\section{Collateral Number and Length Increase Transiently during Nerve Terminal Growth}

Tissue from neonatal $(\mathrm{P} 2,3,4,6,9)$ and mature $(\mathrm{P} 30)$ mice was processed and imaged using serial block-face scanning electron microscopy (SBEM) to bracket initial growth of the calyx of Held $(\mathrm{CH})$ nerve terminal at $\mathrm{P} 3$, resolution of competition to achieve monoinnervation onto most cells (88\%) by P9, and a mature end point (Kandler and Friauf 1992; Holcomb et al, 2013). Light microscopy showed prominent collaterals from the $\mathrm{CH}$ during growth (Kandler and Friauf 1992; Morest 1968). We provide the first 3D volumetric views of calyx collaterals at high spatial and daily temporal resolution, to characterize them by internal structure and contact with neurons and glia as the neural circuit forms. $\mathrm{CH}$ terminals here were segmented and those with largely complete collateral networks were skeletonized to measure the length and number of these arborizations across postnatal development $(n=2970$ collaterals, 34 CHs; Fig. 1A $1-5$, C'). The number of collaterals increased with size of the $\mathrm{CH}$, measured as apposed surface area with the postsynaptic membrane (Fig. $1 \mathrm{~B}, \mathrm{r}^{2}=0.79, \mathrm{~m}=\sim 11$ collaterals/ $100 \mu \mathrm{m}^{2}$ ). The density of short reaching collaterals increased between P6 and P9 (Fig. 1C), despite the majority (75\% P6$88 \%$ at P9) of principal cells having established mono-innervation by these ages (Holcomb et al. 2013). Mature CHs (P30) form a consistent end point, whereby either no (80\%) or one (20\%) collateral innervates a nearby neuron and which volume EM reveals to be of moderate size $(\sim 64$ $\mu \mathrm{m}^{2} ; 16$ synapses within terminal).

We measured a broad range of collateral length $(\sim 1-70 \mu \mathrm{m})$. Only $5 / 21$ collaterals $>40$ $\mu \mathrm{m}$ extended outside of our image volumes such that their length could not be measured, so it is likely that few collaterals exceeded lengths beyond $70 \mu \mathrm{m}$. Median and variance of collateral 


\section{Chapter 2}

length did not change from P3-6 (median values at P3, $5.88 \pm 8.17 \mu \mathrm{m}, \mathrm{n}=245 ; \mathrm{P} 4,6.24 \pm 9.65$ $\mu \mathrm{m}, \mathrm{n}=590 ; \mathrm{P} 6,4.84 \pm 9.53 \mu \mathrm{m}, \mathrm{n}=270$; Holcomb et al. 2013), so the spatial extent of the collateral field is established early and increased in density with age. We combined these ages into a single histogram $(\mathrm{n}=1,352$, median $5.85 \pm 9.32 \mu \mathrm{m}$; Fig. $1 \mathrm{C})$ and used the median value to distinguish the short collaterals from long filopodia and growth cone tipped collaterals. Following the establishment of monoinnervation, $\mathrm{P} 9 \mathrm{CH}$ collaterals were composed predominantly by short filopodia $(95.6 \%<5.85 \mu \mathrm{m})$ with few observable branches $(\mathrm{n}=340$, median $=1.63 \pm 1.99 \mu \mathrm{m})$, and the longest measured was $21 \mu \mathrm{m}\left(\right.$ Fig. $\left.1 \mathrm{~A}_{4}, \mathrm{C}, \mathrm{D}\right)$.

Both filopodial (finger-like) and growth-cone tipped collaterals were shown using light microscopy to emanate from the growing $\mathrm{CH}$ (Morest, 1968b; Kuwabara et al., 1991; Kandler and Friauf, 1993). We show that all short collaterals are filopodial tipped. A minority of long collaterals (124/634) end in growth cones (closed and open arrowheads, respectively in Fig 2A). Few growth cone tipped collaterals remained at P9. Two of five CHs by $\mathrm{P} 9$ retained a small number of growth cone tipped collaterals. These remaining growth cones formed areas of apposition along nearby axonal processes ( 2 of 5) or a dendrite of a nearby principal cell (not shown).

\section{Arrangement of CH Collaterals Facilitate Selective Spatial Overlap}

The developing MNTB is composed primarily of dense populations of principal cells and glia (Dinh et al., 2014; Brandebura et al., 2018). While collaterals enumerate and lengthen during $\mathrm{CH}$ growth, collateral fields from nearby $\mathrm{CHs}$ may occupy an overlapping territory within the 
neuropil (Fig. 3A-C). This was especially true of observations from the first postnatal week (P3, P4 and P6) in which many of the longest $\mathrm{CH}$ collaterals were documented (Figure 2C, 3D) and principal cells were most tightly clustered within the nucleus (Hoffpauir et al., 2006; Holcomb et al., 2013). An intriguing possibility arises that collaterals may signal through contact-mediated communication between presynaptic endings. However, SBEM observation reveals nearby CHs can participate in selective contact with other inputs with either heavily overlapping collateral fields in some cases, or entirely segregated territories in others (Fig. 3E-F).

\section{$\underline{\text { Internal Structure and Contacts of CH Collaterals }}$}

Since EM labels all cells and their intracellular structure, we next investigated differences in the morphology and targets of these three types of endings (short and long filopodial, long growth cone collaterals), and whether they formed synaptic connections. The main body of the $\mathrm{CH}$ contained a large collection of discernable synapses, defined by concentrations of synaptic vesicles near convex regions of the postsynaptic membrane, dark and thickened postsynaptic staining indicative of a postsynaptic density, and locally positioned mitochondria and reticulated membranous structures (Fig. 2B').

Most short collaterals extended within the immediate vicinity of the neuronal soma in regions of the neuropil with notable extracellular space $(76 \%$ at $\mathrm{P} 3,63 \%$ at $\mathrm{P} 4,58 \%$ at $\mathrm{P} 6$; labeled "f" in Fig. 2C). About 1/3 of terminal collaterals extended along the somatic surface and may act to guide $\mathrm{CH}$ expansion along the cell body. Very few short collaterals $(<10 \%)$ contacted neighboring neurons (somata, dendrites or axons) or glia. Short filopodia contained vesicles and 


\section{Chapter 2}

reticulated membrane (Fig. 2C, C'), whether the collateral extended among other neurites and the extracellular space or along a cell body, but did not exhibit features of synaptic contacts.

Long filopodial collaterals also terminated largely among other neurites and extracellular space $(66 \%$ at $\mathrm{P} 3,81 \%$ at $\mathrm{P} 4,73 \%$ at $\mathrm{P} 6)$. Unlike short collaterals, these collaterals more commonly contacted neighboring neurons and glia ( $\sim 30 \%$ at P3, $\sim 15 \%$ at $\mathrm{P} 4$ and $\sim 13 \% \mathrm{P} 6)$ and supported numerous secondary branches (Fig. 2B''). Long filopodia contained many vesicles, reticulated membrane and mitochondria although the latter typically did not extend within $10 \mu \mathrm{m}$ of the tip (Fig. 2D'). This intracellular profile also was independent of terminus target, and did not exhibit synaptic specializations (Fig. 2D, D').

Different still from filopodial collaterals, growth cone-tipped collaterals more frequently contacted neighboring neurons and glia at P3 (50\%) and P4 (46\%), but less frequently at P6 (15\%). Conversely, growth cone contacts with other $\mathrm{CH}$ collaterals from nearby terminals increased during the same period (P3 19\%; P4 43\%; P6 45\%). Growth cone collaterals and their filopodial extensions contained vesicles and reticulated membrane. Mitochondria were present up to the central domain at the base of the growth cone (Fig. 2E, E'), however no evident features of synaptic specializations.

At P9, CHs had very few long collaterals $(4.4 \%$ of $340>6 \mu \mathrm{m}$; Fig. 1 A4, C, D). Nearly all were filopodial-tipped (12/15), although growth-cone tipped collaterals were longest. Such a preponderance of simple, short filopodia is suggestive of a net retraction or removal of the longest exploratory processes begins $\sim$ P9. Moreover, only $3 \%$ of the 340 collaterals at P9 (5 
$\mathrm{CHs}$ ) exhibited contact with adjacent cells or CHs. These may represent the limited number of CHs at P30 that extended a single collateral that contacted a neighboring cell (4/23 CHs; Fig. 1 A5) (Morest, 1968a; Kandler and Friauf, 1993).

We next devised methods to observe these tissue dynamics in vitro using brain slices imaged by lattice light sheet microscopy.

\section{Dynamic Imaging of the Developing MNTB}

\section{Calyx Collaterals.}

The diverse morphology and density of the $\mathrm{CH}$ collateral arborizations demonstrated with SBEM at young ages, taken with previous indications that $\mathrm{CH}$ collaterals exhibit observable motility and progressive turnover in vivo (Rodríguez-Contreras et al., 2008), may bely a complex form of presynaptic organization/communication through collateral contact during MNTB circuit formation. We sought to employ lattice light-sheet (LLS) microscopy to volumetrically image $\mathrm{CH}$ development at a rapid time scale within living tissues $(0.3-1.4 \mathrm{~Hz})$ in order to characterize the behavior of $\mathrm{CH}$ terminal arborization. Parvalbumin Cre (PV-Cre) driven tdTom expression provides selective and strong labeling of bushy cell neurons within the ventral cochlear nucleus (VCN; Fig. 4A, A') as well as the associated axonal projections to the respective ipsilateral lateral superior olive and contralateral MNTB (Fig. 4A, A"). PV is not expressed until the second postnatal week by MNTB principal neurons (Kolson et al., 2016). To reveal postsynaptic targets within the MNTB we transfected the MNTB with HDAd to deliver a GFP construct into MNTB 


\section{Chapter 2}

neurons (Fig. 4A, A'). Moreover, the selective presence of retrograded GFP-labeled somata in the VCN confirms td tomato labeled axons of the ventral acoustic stria in the MNTB are originate from the GBCs (arrows Fig 4A'). Acutely prepared tissue was secured to a slatted metal holder following resection of the cerebellum (Fig. 4B) and 4D image volumes were collected from live slices using LLS microscopy under near physiologic conditions (Fig. 4C, D). Light sheet excitation was presented at a $45^{\circ}$ orientation using a custom Bessel pattern with concurrent and fixed orthogonal image detection to produce three dimensional rhomboid volumes (Fig. 4C-C'). Following each imaging session, tissue viability was verified through verification of no obvious blebbing or tissue shrinkage and continued growth throughout each time-series. Repeated imaging sessions (20-90 minutes) were performed on each slice where possible throughout the 4 hour period of tissue viability following rapid decapitation. Subsequently, $\mathrm{CH}$ collateral dynamics were analyzed in an immersive virtual reality (IVR) environment using visualization and annotation software developed within our laboratory (syGlass; www.syglass.io ) (see Methods). Data analyzed in this way provided insight into the dynamic behavior of each of our three classes of $\mathrm{CH}$ collateral structures.

\section{Short Filopodial Collaterals.}

We hypothesized that the short filopodial $\mathrm{CH}$ collaterals maintain a static boundary around immature innervation territories based on the perseverance of these numerous stunted structures throughout the first two weeks of life. The PV Cre driven cytoplasmic tdTomato fluorescence provided robust labeling of calyciferous axons and terminals although fewer labeled collaterals could be dynamically tracked along each developing $\mathrm{CH}$ relative to the more 


\section{Chapter 2}

numerous arborizations revealed with complete reconstruction with SBEM (Fig. 5 maximum intensity projection (MIP) illustrating a single $\mathrm{CH}$ with a number of finger-like collaterals

protruding away from the primary terminal). A variety of motile behaviors were observed among dynamic $\mathrm{CH}$ collaterals. Approximately $29 \%$ of all collaterals with entirely captured extents (16 of 56 collaterals from $11 \mathrm{CHs})$ did not significantly change length $(>2 \mu \mathrm{m})$ during the observation period of any of our experiments. The remaining 40 collaterals extended $(n=32)$, retracted $(n=6)$ or both $(n=2)$, by more than $2 \mu$ m (often doubling/halving overall collateral length) within respective observation periods. Moreover, the relatively short collaterals measured here often exhibited transient lifetimes (11 of 21 short collaterals with lifetimes completely captured within a single experiment persisted $<5$ minutes) but still exhibited significant extension and retraction dynamics (Fig. 5C-E). In one particularly well resolved experiment, adjacent collaterals from the same $\mathrm{CH}$ illustrate unsynchronized extensions and retractions of 4-6 $\mu \mathrm{m}$ that further demonstrate the limited extant common among these shortest collaterals $(<30$ minutes; Fig. 5B, B', B' '). Importantly, the highest frequency volume acquisition rates $(\geq 1.3 \mathrm{~Hz})$ during largely halted periods of motility revealed a near constant state of small changes (150-300 nm) in collateral length (Fig. 5C-E). To mitigate jitter in measurement of growth trajectory, a threshold of $3 \mu \mathrm{m}$ of monotonically directed length change was used to filter plots of collateral velocity. These rates varied between 0.1 and $18 \mu \mathrm{m} /$ minute (Fig. 5C'). These resultant velocities at times rivaled some of the fastest measured filopodia and/or axonal growth cone extensions/retractions in studies of neuronal organotypic culture from a variety of model organisms (Bray and Chapman, 1985; Halloran and Kalil, 1994; Bellar et al., 2013; Özel et al., 2015; Stanganello et al., 2015). 


\section{Chapter 2}

\section{Long Filopodial Collaterals.}

Long collaterals $(>5.85 \mu \mathrm{m})$ extend to lengths sufficient to interact directly with a plethora of local principal neurons within a territory several times wider than the $\mathrm{CH}$ terminal itself ( 1 of 4 principal cells innervated at the soma by outgrowths from a single $\mathrm{CH}$ shown in Fig

2A). Fast LLS imaging revealed these long filopodia extend and retract as fast as short filopodial ( $\sim 40 \mu \mathrm{m} / \mathrm{min}$ peak instantaneous velocity, $1.236 \mu \mathrm{m} / \mathrm{min}$ average velocity during imaging session at P9). However, the dense branching observed along the length of long collaterals also underwent dramatic turnover and complex motility that significantly increase the volume patrolled by a single collateral projection. This sequence was often characterized by the rapid formation of proximal branches that rapidly change length and position as though each is anchored at a motile branch point, capable of shifting position along the length of the primary collateral stalk. The emergence of interstitial branches allowed collaterals to contact many adjacent calls within the neuropil $\left(469.7 \mu \mathrm{m}^{3}\right.$ covered in approximately 10 minutes in the example illustrated in Fig. 6). Perhaps unsurprisingly, long collaterals were found to regularly come into direct contact with collaterals from other $\mathrm{CHs}$ due in part to arbor density and the extreme proximity among developing CHs. An example of direct interaction between collaterals depicted in Supplemental Fig 1 illustrates the transient duration of contact, generally lasting only a few seconds per instance, that inevitably led to a change in the trajectory of one or both collaterals participating in this interaction.

\section{Long Growth Cone Collaterals.}

Given the length of many growth cone tipped collaterals (GC collaterals), it was challenging to capture them and their $\mathrm{CH}$ of origin utilizing the reduced field of view 


\section{Chapter 2}

necessitated for fast LLS image acquisition. However, for some CHs, we were able to image long collaterals tipped with growth cones for at least a subset of the imaging session (10 and 22 $\mu \mathrm{m}$ long GC collaterals, outlined in green and purple respectively; Fig. 7A-A'). The growth cone presented in purple (Fig. 7A') exited the volume within one minute of the experiment; the growth cone in green exhibited no net change in collateral length although the shape of the terminal lamellipodium underwent significant reorganization (not shown). The growth cone dynamics reported here were inferred to originate from $\mathrm{CHs}$ situated outside of our restricted field of view in the LLS experiment. In support of this inference, further investigation revealed the PV-Cre driven tdTom expression in pre-hearing animals used in the present study was restricted to the axons of the ventral acoustic stria passing in and through the MNTB in the auditory brainstem. Thick tissue $(300 \mu \mathrm{m})$ surveys using $2 \mathrm{P}$ microscopy yielded no apparent growth cones originating from non-calyceal axons within the bounds of the MNTB that were constitutively labeled with tdTom further reinforcing the specificity of our genetic targeting of bushy cell afferents within the ventral brainstem (Supplemental Fig. 1).

GC collaterals from CHs outside of the restricted ROI of the LLS volumes were more easily monitored without direct observation of their source (Fig. 7B). The motility of GC collaterals appeared stochastic with punctuated periods of extension, retraction and pausing while these dynamics were directly paralleled by highly variable fluctuations in the reflected rate of motility (Fig. 7 C-D). Local GC displacements of up to $20 \mu \mathrm{m}$ were measured by monitoring the advancement of the lamellipodium over time. The average rates of motility peaked in correlation with the expansion of the $\mathrm{CH}$ itself between P2-5 (Fig. 7D; Holcomb et al., 2013; Rodríguez-Contreras et al., 2008). Interestingly, the fastest rates of extension ( $>3 \mu \mathrm{m})$ measured revealed instantaneous growth can be 20 times higher than the corresponding average growth 


\section{Chapter 2}

rate. In fact, the growth cones monitored in the current experiments exhibited unprecedented instantaneous motility as high as $20 \mu \mathrm{m} / \mathrm{min}$ (Fig. 7D-E, F).

Similar to the long filopodial-tipped collateral interactions, growth cones were found to transiently contact other neurites and CHs. Generally, the linear growth of the GC is significantly altered following contact, wherein the GC undergoes dramatic lamellipodial collapse, collateral retraction and subsequent growth in along an altered trajectory (Supplemental Figure 2).

\section{Discussion:}

Neural circuit assembly involves the complex interplay of axonal path-finding, arborization and synaptogenesis to establish appropriate mature connectivity (Cohen-Cory and Lom, 2004; Garner et al., 2006). High resolution microscopic techniques in recent years have been developed that are ideally suited to investigate fine changes in circuit structure within developing tissues. We first applied SBEM to the MNTB to more fully characterize the nature of the postnatal establishment and transformation of the $\mathrm{CH}$. As previously reported, protocalyces produce a complex assembly of collateral arbors that intriguingly persist only during development and ultimately consolidate by maturity to leave a highly fenestrated and often unbranched terminal (Kandler and Friauf, 1993; Rodríguez-Contreras et al., 2008; Holcomb et al., 2013). The collateral field present on protocalyces shown in the present study represent a collection of structures including simple, short collaterals as well as longer, more complex terminal collaterals with either complex filopodia or growth cone-tipped endings that may or may not be exclusively segregated. SBEM has also revealed a greater number of collaterals than could be observed with light microscopy, in part due to finer resolution aiding in the identification of these thin and highly abundant collaterals, and further helps to explain the 


\section{Chapter 2}

discrepancy in previously underestimated frequency of collaterals of less than $5 \mu \mathrm{m}$ (RodríguezContreras et al., 2008). In agreement with previous work, we found that $\mathrm{CH}$ collaterals are present for at least several days following the establishment of monoinnervation, until just after the onset of sensitivity to airborne sound in rodents ( P10) (Kandler and Friauf, 1993; Holcomb et al., 2013). It is tempting to speculate the consolidation of the $\mathrm{CH}$ through retraction of the collateral field may be related to the sculpting process that leads to the stereotypically fenestrated morphology of the mature $\mathrm{CH}$ during this period of development. The loss of $\mathrm{CH}$ collaterals

likely marks the end the critical period defined within this system (Nakamura and Cramer, 2013) especially when considering the reduction in secondary signally capacity these collaterals would otherwise provide (Rodríguez-Contreras et al., 2008). We have found that $\mathrm{CH}$ collaterals form abundant associations with secondary principal neurons within a local volume around the $\mathrm{CH}$, as well as with other collaterals from nearby CHs and/or glial cells (Supp Mov 1). Notably, the conspicuous lack of mitochondria along many of the sites of collateral apposition may be indicative of developmentally transient sites of communication via immature synapses, with most contacts being maintained for only short time periods (Lee and Peng, 2008). However, SBEM reveals a static view into the process of neural circuit formation and inherently prevents the possibility of monitoring the real-time dynamics requisite to the formation or maintenance of complex arborization within a self-assembling neural network.

\section{Dynamic Tissue Reorganization.}

Recent advances in imaging capabilities provided a unique opportunity to monitor tissue dynamics at high spatial and temporal resolution in living tissues. Acute brainstem slices here were applied to the LLS microscope successfully for the first time to reveal high rates of 


\section{Chapter 2}

structural reorganization and motility in living mammalian neural tissue. We found a subpopulation of simple calyceal collaterals, short filopodia, that do not extend great distances

from the edge of the $\mathrm{CH}$. Short filopodial were previously postulated to have limited lifespans on the order of an hour (Rodríguez-Contreras et al., 2008) but we observed similar extension and retraction dynamics could limit the existence of some collaterals to less than 5 minutes.

Moreover, these often short-lived collaterals could achieve extension and retraction rates of nearly $20 \mu \mathrm{m} / \mathrm{min}$. Notably, these dynamics were asynchronous among the collaterals from each $\mathrm{CH}$ and permitted each terminal to maintain a collateral field size of several to tens of microns, irrespective of the absolute size of the terminal.

Long calyceal collaterals were considerably more complex in both morphology and behavior. These collaterals were more exploratory in nature during live cell imaging and extended significantly further from the $\mathrm{CHs}$ in which they emerged in both fixed and live preparations. Previous work monitoring calyces in vivo reported peak collateral extension of approximately $20 \mu \mathrm{m} / \mathrm{hr}$ with 2P imaging (Rodríguez-Contreras et al., 2008). Our fast LLS microscopy revealed GC collaterals could sustain an average motility of $1-2 \mu \mathrm{m} / \mathrm{min}$ for tens of minutes at a time while expanding the bounds of the terminal territory. Often the long collateral dynamics remained highly variable throughout the longest imaging sessions (up to 90 minutes). Long filopodia had a less directed growth trajectory relative to the more linear motility of growth cone-tipped arbors. Complex branching of long filopodia further differentiated these structures as these collaterals rapidly extended branches that dramatically swept through tissue to cover relatively large volumes of the neuropil. This "patrolling” behavior creates a larger zone that defines the extent of the terminal territory and may be important for spatial organization as these structures have been shown to be sites of transient calcium signaling (Rodríguez-Contreras et al., 


\section{Chapter 2}

2008). Moreover, for the first time we have observed interactions between collaterals from different $\mathrm{CHs}$ that may demonstrate a contact-mediated repulsive signaling behavior that prevented the advance of collaterals from encroaching $\mathrm{CHs}$ and may further act to organize the innervation territory for proper frequency tuning during neonatal growth and competition among developing protocalyces. Growth cone-tipped collaterals were found to exhibit a net extension in nearly all recorded observation sessions, which suggests these collaterals are responsible for actively exploring and seeking new innervation territory within the MNTB. It is possible this type of pathfinding and synapse forming behavior in collateral growth cones aids directly in the stereotyped establishment of proper frequency tuning through selective synapse formation with postsynaptic partners during the postnatal period of $\mathrm{CH}$ growth. Specifically, the initial surge in terminal growth between 2 and 3 days after birth coincides precisely with the arrival of spontaneous auditory activity derived from purinergic signaling in the neonatal cochlea (Tritsch et al., 2007, 2010). Thus, the onset of bursting activity could serve as a permissive developmental signaling regime for the expansion of the protocalyx and its associated collateral network in rodents via a Hebbian-like mechanism that promotes the growth of the $\mathrm{CH}$ along the soma of a MNTB principal neuron.

\section{Peak Dynamics of Calyx Growth.}

Importantly, exploratory extension and retraction of collaterals is generally thought to represent a transient phenomenon aiding in the precise establishment of mature connectivity (Ruthazer et al., 2010). Our previous work using SBEM revealed that peak expansion of the protocalyx occurs between P3 and P4 in mice (Holcomb et al., 2013). Similarly, in rats it has been shown that $\mathrm{CH}$ collaterals are most abundant and reach the greatest extent between $\mathrm{P} 3$ and 


\section{Chapter 2}

4 in agreement with the present study in mice (Kandler and Friauf, 1993; Rodríguez-Contreras et al., 2008). The high temporal and spatial resolution of the LLS was effective in revealing that high level of motility over short periods of time among calyceal collaterals, further enriching our understanding of these presynaptic processes. Multiple studies show that precise presynaptic innervation of appropriate targets is achieved through a complex interaction between temporally regulated chemoattraction (Mortimer et al., 2008; Poon et al., 2013) synaptic activity (Tashiro et al., 2003; Chen et al., 2013; Massobrio et al., 2013), structural organization of the stratum or microenvironment (Rajnicek et al., 1997; Weigel et al., 2012), and intrinsic transcriptional programs (Koundakjian et al., 2007; Lallemend et al., 2012) that all serve to regulate the extent and nature of neuronal connectivity. Many, if not all of these aforementioned mechanisms may have differential effects over time but likely serve to initiate the bulk of cellular reorganization necessary to sculpt the auditory system ahead of the sensitivity to external sound.

\section{Critical Period for CH:MNTB Formation.}

Several factors likely culminate in the postnatal MNTB to provide a permissive environment for large terminal growth. However, as this critical period concludes and many neural circuits achieve their mature connectivity, extracellular matrix (ECM) proteins begin occupying the spaces between cells. Some of these ECM proteins, such as sulfate proteoglycans, inhibit growth cone advance and filopodial number (Bellar et al., 2013) although others such as laminins promote the growth of axons expressing appropriate subtypes of integrin receptors (McKerracher et al., 1996). Establishment of the ECM may in part act to limit the capacity for $\mathrm{CH}$ collateral maintenance and end the period of dynamic growth and reorganization by limiting the amount of free extracellular space available within the microenvironment (Tashiro et al., 


\section{Chapter 2}

2003). Just after P3 within the MNTB, ECM molecules including aggrecan, brevican and hapln1 slowly begin the protracted process to coalesce in the spaces between cells and reduce available area for presynaptic motility (Kolson et al., 2016). In addition to reduced motility through ECM formation, the microglia population in the MNTB also increases by the end of the first postnatal week (Cramer and Rubel, 2016). These infiltrating macrophages have been shown to inhibit neurite outgrowth and stimulate collateral retraction in other parts of the nervous system (Busch et al., 2009) and may further help to explain the reduction in collateral motility, length and number by the end of $\mathrm{CH}$ development.

\section{Conclusion.}

Recent advances in gentle and fast volumetric imaging allow for the observation of rapid tissue dynamics in thick mammalian tissue explants. We have found $\mathrm{CH}$ collateral arborization can be structurally reorganized over an unprecedented timescale of seconds to minutes and their dynamic interaction raises interesting signaling questions for future research. Additionally, acute brainstem slices are inherently difficult to maintain in culture limiting acute imaging sessions here to less than three hours per experiment to allow even neurites severed from their cell bodies to continue to grow before noticeable degeneration of the tissue (Harris et al., 1987). Recent advances in culturing techniques such as media supplementation with a targeted set growth factors (Kronander et al., 2017) paired with chronic LLS microscopy may reveal detailed insight into the initial formation of the protocalyx over longer periods in future studies. In conclusion, we have presented an in vitro model highlighting the complex cellular reorganization undergone by a developing neural circuit at high spatial and temporal resolution. 


\section{Materials and Methods:}

Ethical Approval. The Institutional Animal Care and Use Committees of both West Virginia University and Janelia Research Campus, Howard Hughes Medical Institute, approved all procedures involving animals in accordance with the United State Public Health Service and the United States National Institutes of Health guidelines.

Animals. Mice were maintained in two facilities, West Virginia University and Janelia Research Campus, on a 12-hour light/dark cycle. Homozygous reporter mice (rosa-tdTomato or Ai9; Jackson Laboratory) were crossed with homozygous parvalbumin Cre-recombinase mice (PV-Cre; Jackson Laboratory) to produce offspring that we refer to as PV-tdTom. A separate colony of FVB (FVB/NJ; The Jackson Laboratory) mice were maintained at the vivarium of West Virginia University. Following overnight pairing for timed-mating, pregnant mice were monitored twice daily to ensure ages of pups following birth are accurate to within 12 hours.

Animal Surgery. P0 PV-tdTom mice were anesthetized by hypothermia for six minutes. Afterwards, a drop of Marcaine (0.5\%) was placed on the scalp and animals were placed onto a clay-filled aluminum block that had been kept on ice, with the skull oriented into approximately the horizontal plane. A small incision was made in the scalp with a $26 \mathrm{G}$ needle to expose the skull. Coordinates for lambda and bregma were determined using an injection needle lowered near the skull and held in a Kopf stereotaxic frame (model 940 digital; David Kopf Instruments, Tujunga, CA). The following injection coordinates were used (in $\mathrm{mm}$ ) relative to bregma in 


\section{Chapter 2}

order to target the (MNTB): medial-lateral, 0.3; anterior-posterior, 5.2; dorsal-ventral, 4.2. The injection needle was filled with a solution containing a recombinant adenovirus (rAd) optimized for fast, high-level expression of GFP driven with the pUNISHER cassette (Montesinos et al., 2011). A final volume of $0.5 \mu \mathrm{L}\left(0.33 \mu \mathrm{L}\right.$ of $\mathrm{rAd}$ PUN EGFP at $2.39 \times 10^{8}$ viral particles/ $\mu \mathrm{L}$ stock mixed with $0.17 \mu \mathrm{L}$ of $20 \%$ mannitol in storage buffer containing $10 \mathrm{mM}$ HEPES, $250 \mathrm{mM}$ sucrose, and $1 \mathrm{mM} \mathrm{MgCl} 2 ; \mathrm{pH} 7.4$ ) was injected using a calibrated injection capillary (Blaubrand; intraMARK, Wertheim, Germany) at a rate of $0.1 \mu \mathrm{L} / \mathrm{min}$. Once the solution was injected, the glass needle was kept in place for an additional 4 min to allow the dissipation of backpressure before slow removal. Animals were then placed under a heat lamp $\left(\sim 35^{\circ} \mathrm{C}\right)$ to recover. Animals were returned to their mothers and monitored to ensure pups began to nurse. Pups were sacrificed for imaging at ages $\mathrm{P} 2-12$.

Live-Slice Preparation and LLS Imaging. Following rapid decapitation, brains were dissected in ice-cold oxygenated low-calcium $(0.1 \mathrm{mM})$ ACSF. Coronal brainstem slices that contained both the ventral VCN and MNTB $(250-600 \mu$ m-thick) were prepared using a Compresstome VF-200 tissue slicer (Precisionary Instruments, Greenville, NC) and immediately transferred to an incubation beaker containing standard ACSF (at $21^{\circ} \mathrm{C}$, continuously bubbled with $95 \% \mathrm{O}_{2}-5 \% \mathrm{CO}_{2}$ ) containing the following (in $\mathrm{mM}$ ): $125.0 \mathrm{NaCl}, 2.5 \mathrm{KCl}, 1.0 \mathrm{MgCl}_{2}, 2.0$ $\mathrm{CaCl}_{2}, 10.0$ glucose, 25.0 $\mathrm{NaHCO}_{3}, 1.25 \mathrm{NaH}_{2} \mathrm{PO}_{4}, 0.4 \mathrm{~L}$-ascorbic acid, 3.0 myo-inositol, and 2.0 Na-pyruvate, $\mathrm{pH}$ 7.3-7.4 (310 mOsm). After $30 \mathrm{~min}$ of incubation, slices were mounted in 1\% low melting point agarose onto a modified zebrafish holder (Simon Watkins, University of Pittsburgh) to allow gas exchange from above and below the slice. The holder was immediately 
transferred to an imaging chamber and actively perfused with oxygenated ACSF (at $37^{\circ} \mathrm{C}$ ) supplemented with additional potassium $(25 \mathrm{mM})$.

Images were acquired using a lattice light sheet (LLS) microscope in the Advanced Imaging Core at Janelia Research Campus (https://www.janelia.org/open-science/advancedimaging-center/instruments/lattice-light-sheet-microscope), at low power excitation (150 $\mathrm{mW}$ at $560 \mathrm{~nm} ; 90 \mathrm{~mW}$ at $488 \mathrm{~nm}$ ). Coherently interfering, dithered Bessel beams were presented through a custom $0.65 \mathrm{NA}, 3.74 \mathrm{~mm}$ working distance water dipping lens (Special Optics, Denville NJ) and collected with a CFI Apo LWD 63x water dipping detection objective (1.1 NA, $3 \mathrm{~mm}$ working distance; Nikon Metrology, Brighton MI). Image volumes (42 $\mu \mathrm{m}, 83 \mu \mathrm{m}, \leq 37$ $\mu \mathrm{m})$ were collected at high speed (0.1-1.4 Hz) with fine spatial resolution (104 nm X $104 \mathrm{~nm} \mathrm{X}$ $369 \mathrm{~nm}$ ) for durations of 30 minutes to three hours.

Image Processing. Image volumes appear shear-transformed due to the movement of the sample along the s-axis during acquisition. These data were first de-skewed to reorient image planes to conventional $\mathrm{x}, \mathrm{y}$ and $\mathrm{z}$ coordinates. Data were next deconvolved with a RichardsonLucy algorithm using an experimentally obtained point spread function at each wavelength with a custom Matlab script (Chen et al., 2014). Deconvolved data were cached into syGlass (Morehead et al., in preparation) for rapid presentation and analysis of 4D images. Trajectory mapping was accomplished by manual placement of reference points at the leading tip of target structures. Resultant 3D points were analyzed with Excel and Matlab scripts.

Confocal Imaging. PV-tdTom mice were anesthetized with an IP injection of Tribromoethanol (Avertin, $250 \mathrm{mg} / \mathrm{Kg}$ ). Animals were perfused with normal Ringer's solution 


\section{Chapter 2}

(room temperature) followed by PBS containing 4\% PFA (Fisher Scientific, City, State). The brain was removed from the skull and post-fixed overnight in $4 \%$ PFA $\left(4^{\circ} \mathrm{C}\right)$. Brains were next transferred to cryoprotectant $\left(30 \%\right.$ sucrose in $0.075 \mathrm{M}$ sodium phosphate buffer, $\left.\mathrm{pH} 7.4 ; 4^{\circ} \mathrm{C}\right)$ for a second overnight incubation. Coronal sections of the brainstem were sliced to a thickness of 40 $\mu \mathrm{m}$ using a freezing microtome (model HM450, Microm, Waltham, MA). Free floating sections containing the MNTB were imaged in a Fluorodish (WPI, Sarasota, FL) on a confocal microscope (model LSM-710, Zeiss, Thornbridge, NY) with either a 10X or 40X objective in order to assess the accuracy and extent of viral transfection within the developing MNTB.

Serial Block-Face Scanning Electron Microscopy. FVB mice were perfused and processed for serial block-face scanning electron microscopy as described previously (SBEM; Holcomb et al, 2013). Briefly, animals were perfused with normal Ringer's solution followed by $0.15 \mathrm{M}$ cacodylate buffer containing 2.5\% glutaraldehyde (Polysciences) and $2 \%$ formaldehyde (Fisher Scientific) with $2 \mathrm{mM}$ calcium chloride. Brains were subsequently drop-fixed in the same solution for an additional 18 hours before sectioning and embedding. Resin embedded samples were shipped to the National Center for Microscopy and Imaging Research (University of California, San Diego) for image acquisition.

MNTB principal cells completely contained within image volumes along with their associated presynaptic innervation were manually segmented using IMOD (University of Utah) and digitally meshed to produce ultrastructural representations of the circuit. Furthermore, these models serve as a histological reference of the fine structure of the $\mathrm{CH}$ collateral field which were difficult to entirely recapitulate using lattice light-sheet microscopy. 3D reconstructions of presynaptic terminals using SBEM were subsequently skeletonized to produce splines 


\section{Chapter 2}

representing each arbor within the $\mathrm{CH}$ collateral network differentially representing fine branching structure of each collateral. The number of collateral arbors were compared to the size of each $\mathrm{CH}$, defined as its apposed surface area or the segmented plane representing the area of direct contact between pre and postsynaptic membranes.

Statistical Analysis. A t-test was employed to assess the Pearson correlation coefficient between $\mathrm{CH}$ size and collateral number. Values reported in the text are the mean $\pm \mathrm{SD}$. 


\section{Chapter 3: Two Types of Somatic Spines Provide Sites for Intercellular Signaling During Calyx of Held Growth and Maturation}

Dakota Jackson $^{1 *}$, Paul Holcomb ${ }^{1 *}$, Mark Ellisman ${ }^{2}$, and George Spirou ${ }^{3}$

${ }^{1}$ Rockefeller Neuroscience Institute, West Virginia University School of Medicine, Morgantown, West Virginia 26506

${ }^{2}$ National Center for Microscopy and Imaging Research, University of California, San Diego, La Jolla, California 92093

${ }^{3}$ Department of Medical Engineering, University of South Florida, Tampa, Florida 33620

${ }^{*}$ Co-first authors 


\begin{abstract}
:
Dendritic spines have been described in developing and mature systems, but similar extensions from cell bodies are less studied. We utilized electron microscopy image volumes, uniquely collected across a range of early postnatal and month-old mice, to characterize and describe two types of somatic processes that extended into and under the developing calyx of Held $(\mathrm{CH})$, which we named type 1 and type 2 spines. Type 1 spines occurred singly, were mostly vermiform in shape, and formed regularly spaced indentations into the $\mathrm{CH}$. Type 1 spines appeared in concert with the earliest expansion of the $\mathrm{CH}$ by $\mathrm{P} 3$, peaked at $\mathrm{P} 6$ and returned to low density at P30. Type 2 spines were intertwined into a secondary structure called a spine mat, which has not previously been described in the CNS, and were more complex geometrically. Type 2 spines formed after the $\mathrm{CH}$ crossed a size threshold, reached maximum density at P9, and were absent from most $\mathrm{CHs}$ at P30. Both spine types, but a higher density of type 1 spines, were sites for synapse formation. Spine mats brought pre- and postsynaptic neurons and glial cells into contact, and were captured in stages of partial detachment and engulfment by the presynaptic terminal, suggesting trans-endocytosis as a mode of removal ahead of maturity. In conglomerate, these observations reveal somatic spines to be sites for chemical neurotransmission and chemical sampling among synaptic partners and glia as tissue structure transforms into mature neural circuits.
\end{abstract}




\section{Chapter 3}

\section{Introduction:}

Neurons communicate via synaptic and non-synaptic mechanisms, often via specialized extensions of pre- or postsynaptic processes into or underlying the adjoining post- or presynaptic partner, respectively. A well-studied and prevalent structure is the dendritic spine which commonly protrudes from dendrites, displays variation in size and shape, and receives predominantly excitatory chemical synaptic input onto the spine head (Nimchinsky et al., 2002; Bourne and Harris, 2008; Gipson and Olive, 2017). In some cases, spines are surrounded by and therefore embedded into presynaptic terminals (Westrum and Blackstad, 1962; Petralia et al., $2015,2018)$. These processes exhibit a range of morphologies, occur in many neural systems across invertebrate and vertebrate species, and are generally termed invaginating projections (for comprehensive reviews, see Petralia et al., 2015, 2018). An example of this arrangement with a complex geometry is the thorny excrescence on the dendrites of hippocampal pyramidal cells, which are enveloped by mossy fiber terminals of dentate gyrus neurons (Gonzales et al., 2001; Popov et al., 2011).

Similar to dendritic spines, invaginating projections can extend into presynaptic terminals from the somatic membrane and be sites for innervation. These structures have been found in the auditory brainstem and vestibular nuclei, where they have been termed somatic appendages. In the cochlear nucleus somatic appendages can embed into the large presynaptic auditory nerve terminals, called endbulbs, that form onto many cells (Cant and Morest, 1979; Tolbert and Morest, 1982; Spirou et al., 2005) and in the medial nucleus of the trapezoid body (MNTB) they embed into the large presynaptic terminal called the calyx of Held (CH) (Morest, 1968a; Sätzler et al., 2002; Hoffpauir et al., 2006; Holcomb et al., 2013). 


\section{Chapter 3}

In addition to single cellular processes, specialized clusters of intertwined processes have been documented extending from and overlying the postsynaptic somatic membrane underneath the large calyx of the chick ciliary ganglion, where each element is termed a spine and their collection is termed a somatic spine mat (Shoop et al., 1999, 2002; Coggan et al., 2005). Somatic spine mats were studied by high resolution electron tomography which permitted description of the complex, branched shape of individual spines. Spine mats form during embryonic development and persist in the mature nerve terminal. To date, this intriguing organization of somatic processes has not been identified at synaptic junctions in the CNS nor in mammals.

In this study we focus on extensions from the postsynaptic somatic membrane that embed into or underlie the presynaptic $\mathrm{CH}$ which, like the calyx in the chick ciliary ganglion, is a large axosomatic terminal that is a useful model system for studying neural circuit formation. This terminal grows rapidly in rodents after postnatal day 2 (P2) as innervation proceeds from multiple, converging axons with small inputs to a single large input onto about $75 \%$ of postsynaptic neurons by P6 (Kandler and Friauf, 1993; Hoffpauir et al., 2006, 2010; Holcomb et al., 2013). Both morphological and electrophysiological maturation of the calyx and MNTB principal cell occur in concert over this time period (Hoffpauir et al., 2010; Rusu and Borst, 2011; Holcomb et al., 2013; Sierksma et al., 2017).

In previous studies we noted, qualitatively, the presence of multiple extensions from the MNTB principal cell somatic membrane into the developing $\mathrm{CH}$, and more complex arrangements of somatic processes (Hoffpauir et al., 2006; Holcomb et al., 2013). Using our unique 
developmental series of volume electron microscopy images, we were able to reconstruct these processes in 3D. We describe single spines and their high prevalence of innervation. We also provide the first description of spine mats in the CNS, reveal that they are a common but transient feature of the developing $\mathrm{CH}: \mathrm{MNTB}$ connection, that they appear to be removed by trans-endocytosis, and that they are also innervated. In conglomerate, single spines and spine mats provide multiple routes for intercellular communication. We suggest that as volume EM techniques become more routine, these small yet often complex structures will be more systematically studied and may be revealed as key features of other developing and mature neural systems. 


\section{Chapter 3}

\section{Materials and Methods:}

Ethical approval. All procedures involving animals were approved by the West Virginia University (WVU) Institutional Animal Care and Use Committee and were in accordance with policies of the United States Public Health Service.

Serial Block-face Scanning Electron Microscopy (SBEM). FVB mice of either sex (FVB/NJ; The Jackson Laboratory; or FVB/NCr; National Cancer Institute) were perfused and processed for serial block-face scanning electron microscopy (SBEM) as described previously (Holcomb et al., 2013) at P2, 3, 4, 6, 9 and 30. Briefly, each animal was perfused with normal Ringer's solution followed by $0.15 \mathrm{M}$ cacodylate buffer containing 2.5\% glutaraldehyde (Polysciences) and 2\% formaldehyde (Fisher Scientific) with $2 \mathrm{mM}$ calcium chloride. Following dissection, brains were drop-fixed in the same fixative solution for an additional 18 hours before sectioning for en bloc staining (Deerinck et al., 2010) and embedding. Resin-embedded samples were sent to the National Center for Microscopy and Imaging Research (NCMIR, University of California, San Diego) for SBEM image acquisition (3View, Gatan, using FEI or a Zeiss Merlin scanning electron microscopes). Image volumes were located in the medial MNTB to minimize tonotopic bias and were sectioned at a thickness of 60 or $40 \mathrm{~nm}$ (P9). Resulting SBEM volumes varied by sample in section thickness (P3: $55 \mathrm{~nm}$; P4: $57 \mathrm{~nm}$; P6: $53 \mathrm{~nm}$; P9: $36 \mathrm{~nm}$; P30: $40 \mathrm{~nm}$ ) and pixel dimensions (P3: $12 \mathrm{~nm}$; P4: $10 \mathrm{~nm}$; P6: $4.9 \mathrm{~nm}$; P9: $4.8 \mathrm{~nm}$; P30: $4 \mathrm{~nm}$ ) (previously reported in Holcomb et al., 2013) and each volume contained between 18 and 70 complete cell bodies.

Segmentation and Three-Dimensional Reconstruction. Principal cells and their associated inputs fully contained within SBEM image volumes were segmented using the IMOD 3D reconstruction software (Boulder Laboratory for 3-D Electron Microscopy of Cells, University of 


\section{Chapter 3}

Colorado, Boulder; http://bio3d.colorado. edu/imod/) and subsequently exported to and visualized in 3D using syGlass (IstoVisio, Inc.; www.syglass.io). A total of 44 cells and 60 presynaptic terminals were segmented across our timeframe of interest (P3, 4, 6, 9, and 30; Table 1). Somatic appendages that extended into the calyx of Held nerve terminal were classified as type 1 spines occurring singly or type 2 spines that comprise spine mats. These structures were segmented across serial sections and reconstructed in 3D using the meshing tool in IMOD. Subsequently, reference points (located each at the base of somatic attachment and along the tip of each branch) and associated spine segmentations were converted to a 3D file format using the imod2OBJ command. Exported OBJ files were used in subsequent quantitative analysis.

Morphological features of spines. OBJ files, each containing the meshed type 1 and type 2 spines, and for type 1 spines the base and tip coordinates, were imported into the Rhinoceros CAD software (McNeel, Seattle, WA) for morphological analysis. A custom Python script, using the rhinoscriptsyntax module with built-in commands within Rhino, was run on each spine to quantify the surface area and volume of each spine. For type 1 spines, error correction was then applied by filtering objects with volumes less than $5 \%$ of the mean type 1 spine volume for that terminal; these objects were excluded because they resulted from errors in segmentation or the IMOD meshing process. Additional error checking by inspection in 3D VR (syGlass, IstoVisio) ensured that each unbranched type 1 spine possessed exactly one base and one tip point. Within Rhino, twenty contours representing the relative profile of each type 1 spine were then created at equidistant points along the vector created using the base and tip points. The center of each of these contours was then used to create a center curve for the type 1 spine, which was the measure for the length of the type 1 spine. Center curves that deviated more than $200 \%$ in length from the 


\section{Chapter 3}

direct linear distance from base to tip point were inspected visually. If the deviation proved to be an error in the contouring process $(1.5 \%)$, the linear distance from base to tip point was used as an approximate length instead. This methodology could not be applied to the highly branched type 2 spines, so we do not report total lengths for these spines. The area of near juxtaposition between the pre- and postsynaptic plasma membranes was measured as the apposed surface area (ASA), a metric used previously to assess the relative maturation of the terminal growing over the PC soma (Holcomb et al., 2013), and included the tight association of type 1 spines beneath $\mathrm{CH}$ terminals.

Complexity analysis. In order to quantitatively analyze the complexity of type 1 and 2 spines, as well as compare complexity between these two process types, we computed the convex hull volume for each reconstruction. Spine mesh objects were imported into RhinoCAD, the convex hull was created for each individual process using the ConvexHull Rhino plugin (MelonSoft) implemented with a custom Python code. Following this step, the volume was recorded for both the original mesh, as described above, and the convex hull. We then calculated the ratios of convex hull volume to the original spine volume and plotted these ratios against the original volumes to quantify complexity as a function of spine size.

Statistics. Unless otherwise stated, values are reported as mean \pm standard deviation. Statistical significance across multiple groups was evaluated with a Kruskal-Wallis Test with Nemenyi post-hoc test or ANOVA with a Pairwise Mann Whitney test depending on whether all data being compared was normally distributed. All statistical outcomes of multiple groups were 
Chapter 3

Bonferroni corrected. Single pairwise comparisons were performed with the Mann-Whitney Utest. 


\section{Chapter 3}

\section{Results:}

\section{The shortest somatic processes are located under the calyx of Held}

The transition of neurons in the medial nucleus of the trapezoid body (MNTB) from a smooth somatic surface at $\mathrm{P} 2$ to a rougher texture with multiple somatic processes of varying lengths occurs during growth of CHs and establishment of mono-innervation (postnatal days (P)3-9), as previously observed (Holcomb et al., 2013). Therefore, we initially characterized features of the somatic surface qualitatively during this time frame as well as a post-maturational time point (P30). By P4, 3D reconstructions reveal the longest somatic processes to be outside of the locations for innervation (white arrows in Fig. 1A, A'). These long processes extended in all directions and may be curved or branched. The somatic surface in contact with the overlying $\mathrm{CH}$ (indicated by the ASA in translucent blue, green and orange in Fig. 1A, A') also exhibits somatic processes (red arrowheads in Fig. 1A, A'), although these are notably shorter. At P6, when the $\mathrm{CH}$ is larger, the density and length of long processes outside of the ASA appears to be greater and many are located along the edge of the $\mathrm{CH}$ (white arrows in Fig. 1B, B'). Shorter somatic processes within the border of the ASA appear similar in length but occur at higher density than at $\mathrm{P} 4$ and $\mathrm{P} 3$ and with regularity in their spacing.

\section{Somatic processes underlying the $\mathrm{CH}$ are classified into two groups}

We noted that a subset of somatic processes within the ASA occurred singly, whereas others emerged nearby one-another and intertwined over the somatic surface (red arrows in Fig. 1B, B'). For reasons developed in this and later sections, we named them spines and grouped them into two populations. Single spines extend mostly orthogonal to the cell body surface and directly embed into the $\mathrm{CH}$ (Fig. 1C, arrowheads; Fig. 1C' single spine outlined in red). Clusters 


\section{Chapter 3}

of intertwined spines form a composite structure, which we term spine mats after the homologous description of the spine mat in the chick ciliary ganglion (Shoop et al., 2002), that either extends into the $\mathrm{CH}$ or forms a dome-shaped tangle within a larger recess beneath the $\mathrm{CH}$ (Fig. 1C, arrows; Fig. 1C', spine mat dome outlined in red).

We next asked if single spines could be quantitatively differentiated from the individual intertwined spines of spine mats (Table 1). At each age, multiple cells were reconstructed and analyzed along with their largest or, for cells contacted by competing inputs, their two largest terminals (Table 1). Overall, a total of 44 cells across ages were completely analyzed and quantified (28 PCs with single CHs, 16 with competing pairs), yielding a total of 3373 individual spines. To reconstruct spine geometry in $3 \mathrm{D}$, the bases were defined as the inflection in the curvature of the membrane away from the somatic surface (red dashed lines in Fig. 1C', C',), and structures were segmented across every serial EM section. Notably, nearly all single spines were unbranched (2062/2076 spines). Short single spines had relatively equal width and length (Fig. 2A-A", B-B"; 3D reconstructions and two EM images shown for each single spine). Longer single spines either immediately turned to follow the somatic surface (Fig. 2C-C”) or extended deeper into the $\mathrm{CH}$, typically perpendicular to the cell body surface, and nearly reached the nonsynaptic face of the CH (Fig. 2D-D”). Only a very small (8/2076) subcategory had an expanded head (Fig. 2E-E', F-F') that resembled dendritic mushroom spines. Single spines diameters ranged from 200-500 $\mathrm{nm}$ and lengths ranged from 0.011-3.51 $\mu \mathrm{m}(\mathrm{n}=2076$; Fig. 2G). Mean length increased by nearly $20 \%$ from P3-P6, then decreased at P9 (both increase and decrease were statistically significant; Suppl. Fig. 1, Suppl. Table 1) and P30 due to the absence of longer 


\section{Chapter 3}

spines (P3: $0.758 \pm 0.40 \mu \mathrm{m}, \mathrm{P} 4: 0.835 \pm 0.44 \mu \mathrm{m}, \mathrm{P} 6: 0.887 \pm 0.46 \mu \mathrm{m}, \mathrm{P} 9: 0.610 \pm 0.27 \mu \mathrm{m}, \mathrm{P} 30$ :

$0.621 \pm 0.36 \mu \mathrm{m} ;$ overall $0.752 \pm 0.41)$

Spine mat complexity is best understood in 3D reconstructions. One of the more complex spine mats, as defined by the number of component spines (range: 2-34 spines), illustrated a range of differing spine geometries (19 spines illustrated in Fig. 3). Most spines were branched (examples are \#2, 12, 15 in Fig. 3A). Unbranched spines had multiple bends and/or irregular swellings (\#1, 9, 17 in Fig. 3A) or occasionally appeared vermiform, like single spines (\#4, 8, 11, 16, 18, 19 in Fig. 3A). Variability in interdigitated spine morphologies precluded a direct comparison of length with vermiform single spines. The $\mathrm{CH}$ and an astrocytic process (blue overlay and uncolored cellular elements with asterisk, respectively, in Fig. 3B, C) also can extend processes into the spine mat creating sites for triadic physical contact among pre- and postsynaptic neurons and glial cells.

We next compared single and intertwined spines by fitting their surface with a three-dimensional convex hull (akin to throwing a thin sheet over the structure, Fig. 4D-F), and computed a complexity score as the ratio of the volume of the convex hull to the volume of the spine. Curved and branched structures should have smaller volumes than their convex hull volumes (red or blue structures and translucent overlays, respectively in Fig. 4D-F). Nearly all spines (one exception) larger than $0.6 \mu \mathrm{m}^{3}$ were elements of spine mats (blue symbols in Fig.4A-C). Spines selected at regular intervals across the total population ranked by size at each age $(\mathrm{P} 3,6,9)$ illustrate that the largest somatic processes in the rank order are those that comprise spine mats (blue structures at the right of Fig. 4D-F). Where spine volumes overlapped, especially at P3 and P6, intertwined 


\section{Chapter 3}

spines were more complex than single spines (see insets illustrating single vs. intertwined spines $<0.2 \mu \mathrm{m}^{2}$ : P3: $1.27 \pm 0.2$ vs. $1.69 \pm 0.2$, P6: $1.06 \pm 0.02$ vs. $1.95 \pm 0.6$, P9: $1.19 \pm 0.2$ vs. $1.49 \pm 0.3$; $\mathrm{p}<0.0001$ for all pairwise comparisons Mann-Whitney U-test (M-W); Fig. 4A'-C'). Comparisons across all spines at each age also differed significantly (Fig. 4). The small number of single spines with a higher complexity score than intertwined spines of similar volume represented vermiform spines that had a substantial bend along their length, thus increasing the convex hull volume. Both single and intertwined spines decreased in complexity with age (single spines: P3 vs P9, p<0.05; P6 vs P9, p=0.05; intertwined spines: P3 vs P9, p <0.001; P6 vs P9, p < 0.001; KW). Based on these quantitative differences between the two spine types we henceforth designated single somatic spines as type 1 spines and intertwined spines as type 2 spines.

\section{Prevalence of type 1 somatic spines and spine mats is developmentally regulated}

Previous studies have shown many of the morphological stages of $\mathrm{CH}$ formation occur largely during the first week of life and ahead of the onset of hearing in mice (Kandler and Friauf, 1993; Taschenberger et al., 2002; Hoffpauir et al., 2006; Holcomb et al., 2013). However, auditory neurons continue to undergo changes to intrinsic and synaptic properties until the system nears maturity as early as P30 in both brainstem nuclei (Müller et al., 2019) as well as within the auditory cortex (Carrasco et al., 2013). We next examined the prevalence of type 1 spines and spine mats across principal neurons of the MNTB at early postnatal ages $(\mathrm{P} 3,4,6,9)$ in relation to young adult mice $(\mathrm{P} 30)$. 3D reconstruction shows that at $\mathrm{P} 3$, during initial expansion of the $\mathrm{CH}$, indentations from type 1 spines are rare but occur both in the center and at the edges of the $\mathrm{CH}$ (orange in Fig. 5A). At P6 the density of indentations increased (Fig. 5B), when type 1 spines occur more evenly across the $\mathrm{CH}$. Spine mats (pink, Fig. 5B; arrow indicates spine mat 


\section{Chapter 3}

expanded in Fig. 3) occurred in both central and peripheral regions of the $\mathrm{CH}$ but were not as evenly distributed. At P30, fewer type 1 spines were observed, and spine mats were a rare occurrence. Note that the $\mathrm{P} 30 \mathrm{CH}$ is fenestrated into stalks and swellings, and most swellings do not contain type 1 spines (Fig. 5C).

For a given age, type 1 spine number increased linearly with $\mathrm{CH}$ size, as defined by ASA (Fig. 6A). The slope of each linear fit increased between P3-6, remained similar at P9 and P30, but the intercept at P30 was less, indicating a reduction in type 1 spine number. Normalizing these counts as type 1 spine density revealed a significant age dependence between time points (Fig. $6 \mathrm{~B} ; \mathrm{p}<0.0001 ; \mathrm{K}-\mathrm{W})$. These data confirm that type 1 spines are a feature of initial $\mathrm{CH}$ growth (only 1/12 CH had no type 1 spines), their density increased significantly during the period of postnatal terminal expansion ( $\mathrm{P} 3$ vs $\mathrm{P} 6$; $\mathrm{p}<0.0001 ; \mathrm{K}-\mathrm{W}$ ), and density decreased by $\mathrm{P} 30$ to levels similar to P3 (Fig. 6B, yellow; P9 vs P30: $\mathrm{p}<0.01$, P3 vs P30: p=0.987; K-W).

Spine mats occurred in lower numbers than type 1 spines across all sampled ages (note difference in ordinate scale in Fig. 6A, C), and were rarely found on $\mathrm{CHs}$ below a threshold size ( 2 of $60 \mathrm{CHs}<\sim 100 \mu \mathrm{m}^{2}$ in ASA). As with type 1 spines, the number of spine mats was linearly related to $\mathrm{CH}$ size (Fig. 6C). The slope of this relationship was similar from P3-9, but the intercept was much higher at P9, indicating a significantly greater density of spine mats (Fig. 6D; P9: $\mathrm{p}<0.001$; Kruskal-Wallis Test) that peaked within this age range later than type 1 spine density. At P30, only one spine mat was detected across five terminals. Spine mats were comprised of variable numbers of type 2 spines (median $=6$, mean $=6.8 \pm 5.0$ ). Cumulative distribution functions for each age revealed a transition to larger numbers of type 2 spines per 


\section{Chapter 3}

spine mat between P4-6 (shift in 50\% value of cumulative distribution; Fig. 6F), and a likely reduction at P30 (4 type 2 spines comprise the single spine mat; Fig 6F).

\section{Type 1 spine and spine mat prevalence do not differentiate competing inputs}

In this and the remaining sections we consider structural features of type 1 spines and spine mats that provide clues to their functional significance. Given the synchronized increase in type 1 spine density during the growth of the $\mathrm{CH}$ onto PCs and the appearance of spine mats only after CHs have crossed a size threshold, we hypothesized that they may influence competition between terminals. We defined competing inputs using criteria of Holcomb et. al. (2013): 1) at least two distinct inputs with ASA $>25 \mu \mathrm{m}^{2}$ and 2) relative size ratio of inputs $<5$. Sixteen pairs of competing CHs were identified between P3 and P6 (16/34 PCs). Both inputs in a competing pair were often associated with type 1 spines and spine mats (Fig. 7A). Although the number of type 1 spines was greater in larger terminals (predominantly negative slopes in Fig. 7B), the difference in spine density between terminals was significant only at P4 (Fig. 7C; $p<0.05$; Pairwise MW). We then used the reconstructed terminal ASAs to calculate the percentage of ASA on type 1 spines available for innervation. This quantity was calculated both as an aggregate (Table 1, "\% of Terminal ASA on Type 1 Spines") and on a pairwise competitive basis (Table 2) and revealed no statistically significant difference in percentage of ASA on type 1 spines for either competing category (Table 1). When competing terminals were compared pairwise, only the paired terminals at $\mathrm{P} 4$ approached significance $(\mathrm{p}=0.050, \mathrm{~K}-\mathrm{W})$ with the largest terminal at this age consistently having a higher percentage of ASA on type 1 spines than its competitor (Table 2). Due to the restriction of spine mats to terminals $>100 \mu \mathrm{m}^{2}$, they were 


\section{Chapter 3}

only found at P4 and P6 and did not differ among competing inputs (Fig 7D-E). Thus, neither type 1 spine nor spine mat prevalence consistently differentiated competing inputs.

\section{Types 1 and 2 spines provide area for membrane apposition and are sites for innervation}

Regions of membrane apposition are potential sites for non-synaptic communication. We inspected type 1 spines and spine mats and determined they were in contact with the presynaptic membrane along their entire surface. Instances of glial incursions occurred only when spines were located near the edge of the CH (Fig. 2B', B', C', C', Fig. 3).

We next considered that type 1 spines and spine mats could affect synaptic strength if these structures are consistently innervated. Individual examples of similar appendages being innervated have been presented previously (Sätzler et al., 2002; Hoffpauir et al., 2006; Holcomb et al., 2013). The prevalence of innervation was evaluated for a single terminal at P9 (29 spines, 32 synaptic contacts) in rat (Sätzler et al., 2002). We tabulated the fraction of type 1 and 2 spines from our large population across age, using accepted criteria for synapse identification in SBEM images (Sätzler et al., 2002; Holcomb et al., 2013; Xiao et al., 2013; Sanculi et al., 2020): 1) parallel orientation of the pre- and postsynaptic membranes, 2) presence of synaptic vesicles near the pre-synaptic membrane $(<200 \mathrm{~nm})$ in more than one serial section, 3$)$ contact of at least one vesicle with the pre-synaptic membrane, and added the 4) conservative requirement that these features are present in at least 3 serial sections ( 2 serial sections shown in Fig. 8A, A'). Since en bloc staining for volume EM can reveal postsynaptic densities (PSD), but not reliably, we did not use this feature as one of our criteria. An excitatory synapse onto the more flattened somatic region of the postsynaptic surface is shown for reference (arrow in Fig. 8E). 


\section{Chapter 3}

Similar to the analysis performed for competing inputs, the percentage of ASA on type 1 spines available for innervation on single dominant inputs was quantified (Table 1, “\% of Terminal ASA on Type 1 Spines"). This percentage increased significantly from P3-P6 (P3 vs. P6: p<0.05, K-W), then declined between P6-P30 (P6 vs P30: $\mathrm{p}<0.01, \mathrm{~K}-\mathrm{W}$ ) to levels similar to P3. These results were similar when competitive and dominant inputs were combined. Quantification of synapse prevalence revealed most type 1 spines are functionally innervated at P3 and P4 (> 65\%) and this value reached a maximum of 95\% of spines innervated by P30 (Fig. 8F), even though type 1 spine density had declined significantly (Fig. 6B). Most type 1 spines were innervated at their base, but synapses could also form along their length or near their tip (arrowheads in Fig. 8A-C). Most spines were innervated by one or two synapses (Fig. 8G). Some non-innervated spines had very few synaptic vesicles in proximity to their presynaptic membrane (red outline in Fig. 8D, E). The type 1 spine cytoplasm was largely devoid of heavy metal-stained structures. This "exclusion zone" for cellular organelles, first described here, could extend to a boundary between 50-200 nm below the postsynaptic cell membrane, and laterally away from the spine (dotted lines in Fig. 8A-E).

Since type 2 spines were interwoven into spine mats underneath the $\mathrm{CH}$, much of their surface was not in direct contact with the $\mathrm{CH}$ (Fig. 9). Survey of spine mats across each age revealed $50 \%(535 / 1070)$ of type 2 spines exhibited sites of synaptic contacts. Synapses could occur at the base of type 2 spines (synaptic contact area illustrated in blue; Fig. 9I) but were most frequently located along their length (red arrowheads Fig. 9C-F, H; nearby somatic synapses indicated with arrows, Fig. 9A-B, E-F, H). In a small percentage of spine mats (10\%), the $\mathrm{CH}$ extended 


\section{Chapter 3}

intertwining processes into the core of the spine mat; however, these processes rarely form synapses (not shown). We counted the number of synapses per spine and averaged that number for individual spine mats (each spine mat is a data point in Fig. 9J). The density of synapses per type 2 spine per spine mat was maintained between P3 $($ mean $=0.76 \pm 0.12)$ and P6 $($ mean $=$ $0.80 \pm 0.11 ; \mathrm{p}=0.999 \mathrm{~K}-\mathrm{W}$ ) but significantly increased by P9 (Fig. 9J; mean: $1.27 \pm 0.06 ; \mathrm{p}=$ $0.01 ; \mathrm{K}-\mathrm{W}$ ) when the incidence of spine mats was highest (Fig. 6D). Inspection of the interior of type 2 spines revealed a higher prevalence of labeled cytoplasmic structures than type 1 spines. These labeled organelles were often reticulated membrane that could appear contiguous with reticulated membrane in the main compartment of the cell body (red arrows in Fig. 9B-D, pink and green spines). Other structures found in type 2 spines were primarily bar-shaped and appeared round when transversely sectioned. Unlike the exclusion zone observed under type 1 spines, larger organelles such as mitochondria were found to extend to the edges of the somatic membrane located at the base of type 2 spines (Fig. 9 A, E and F) and in a few cases extended into the spine (not shown).

\section{Trans-endocytosis of spine mats into the presynaptic terminal}

Spine mats at the earliest ages (P3, 4 and 6) exhibited a consistent morphology of tightly interwoven components within a shallow dome beneath the $\mathrm{CH}$. Moreover, nearly all components of the spine mat, be they glial or neuronal (pre- or postsynaptic) can be tracked to a larger process or a cell of origin within the volume. However, concurrent with the dramatic increase in spine mat density at P9 was the appearance of spine mats comprised of multiple elements that are not attached to a cell of origin (green structures in all panels of Fig. 10; all attached type 1 and type 2 spines depicted in orange and pink respectively). All spines were 


\section{Chapter 3}

reconstructed under CHs for five cells at P9 located near the center of the image volume. Three clusters of non-attached elements in the leftmost terminal in Figure 10A illustrate sequential stages of a spine mat removal mechanism. Spine mats could be comprised of intertwined type 2 spines (pink), $\mathrm{CH}$ (blue) and glial (unlabeled) processes, and a small number of elements that were not attached (green) to a cell (3 type 2 spines and 1 non-attached structure in Figure 9B). Other spine mats could be associated with a larger number of non-attached elements relative to few remaining attached elements (fletched arrows in Fig. 10A, Fig. 10C). In these cases, the attached type 2 spines appeared thinner than most other type 2 spines (compare Fig. 10C to Fig. 10B), and they interdigitated into aggregations of detached spines of various sizes that were shifted deeper into the CH (Fig. 10C-C', ). A third group of structures consisted of a complex of fully detached components that were enclosed entirely within the $\mathrm{CH}$ (simple arrows in Fig. 10A, Fig. 10D). To ascertain the generality of this observation, we surveyed an additional 20 cells in this image volume and found partially and fully detached elements associated with $\mathrm{CH}$ innervation on $13 / 20$ cells. We then selected spine mats that appeared to be in different stages of dissociation and quantified the percentage of spine mat volume in contiguous detached elements, which formed a continuum of values (expressed as percentage: 4, 8, 13, 19, 26, 33, 39, 43, 48, $53,57,70,75,91,94)$. This sequence of image volumes indicates a process of trans-endocytosis of entire spine mats into the $\mathrm{CH}$ which may serve to regulate their numbers, among other functions. As the percentage of dissociated elements increased, these elements were located further away from the presynaptic membrane toward the interior of the $\mathrm{CH}$. 


\section{Chapter 3}

\section{Discussion:}

\section{Summary}

Using manual segmentation of SBEM images, we present the first systematic description and differentiation of two types (called type 1 and type 2 ) of specialized spines that emanate from postsynaptic somata and embed into the pre-synaptic terminal of the $\mathrm{CH}$. We make the first observation of spine mats in the CNS and reveal that the incidences of type 1 spines and spine mats follow similar yet distinguishable developmental trajectories. Several other observations support functional roles for intercellular communication. Type 1 spine membranes are contiguous with the presynaptic membrane along their entire surface, type 1 and 2 spines form synaptic connections, and type 2 spines are trans-endocytosed into the presynaptic terminal. Type 2 spines, in contrast to type 1, contain reticulated membrane and other labeled structures, and intertwine with glial and presynaptic processes, with some exposure to extracellular space. Combined, these findings suggest roles for type 1 spines and spine mats in intercellular communication relevant to $\mathrm{CH}$ formation, growth and maturation.

\section{Application of volume electron microscopy to study of somatic processes}

Post-synaptic processes that form invaginations in their synaptic partners were discovered using electron microscopy, as this technique affords the requisite resolution to observe structures with dimensions of tens to hundreds of nm in diameter (for review see Spacek and Harris, 2004; Popov et al., 2011; Petralia et al., 2015; Sanculi et al., 2020) with good tracking of cell membranes, staining of intracellular contents, and visualization of all cellular elements. The intertwined nature of spine mats poses additional challenges; the first description of this structure required the use of high resolution electron tomography to resolve individual structural 


\section{Chapter 3}

components (Shoop et al., 2002). SBEM, as well as other modern volume EM techniques, offers the opportunity to track the distribution of small processes across the entire somatic surface by collecting many hundreds to thousands of serial sections. Acquiring a large tissue volume involves the tradeoff between the size of the volume and resolution. These image volumes have pixel dimensions that ranged from 5 to $12 \mathrm{~nm}$, and as such cytoskeletal elements, particularly microfilaments, could not be identified reliably so these were not quantified. En bloc staining for SBEM provides reliable membrane staining, supporting our report of few vesicles and other membrane-enclosed objects within type 1 spines and a membrane-free zone at their base, but is less reliable in labeling protein clusters such as post-synaptic densities. An advantage of blockface imaging over serial section reconstruction is good alignment across images, which is essential to achieve accurate measurements of surface area, length, and volume from small and, in the case of type 2 spines, tortuous structures, particularly over long distances.

\section{At least three categories of somatic appendages on MNTB neurons}

We previously noted in our volume EM series the extension of processes from the somatic surface, coincident with initial $\mathrm{CH}$ growth between P2-3, that were longer than the somatic spines described here and that transformed the smooth somatic surface into a more "fuzzy" appearance (Hoffpauir et al., 2006; Holcomb et al., 2013). The location of many longer processes along the edge of larger CHs (most evident in Fig. 1B-B') may impose a barrier to further growth and regulate the size of the terminal. These longer somatic processes were noted in early Golgi studies of the developing MNTB (Morest, 1968a, 1968b), and will be treated in a separate report that also encompasses dendrite formation. Type 1 spines have been observed previously in ultrastructural studies of large somatic terminals onto cat bushy cells and perinatal mouse 


\section{Chapter 3}

MNTB, and were referred to as somatic appendages or spine-like protrusions (Cant and Morest, 1979; Tolbert and Morest, 1982; Sätzler et al., 2002; Hoffpauir et al., 2006). Spine mats were not previously observed, nor were the more complex geometries of their constituent type 2 spines. Despite the later appearance developmentally of spine mats relative to type 1 spines, tight adhesion with the associated $\mathrm{CH}$ membrane argues against lateral diffusion and aggregation of pre-existing type 1 spines into spine mats, and instead may be indicative of differential mechanisms leading to the extension of type 1 and 2 spines.

\section{Similarity of type 1 and type 2 spines with other post-synaptic extensions}

Spinules. The thinnest extensions of postsynaptic membrane in the mammalian brain are called spinules and they emanate from dendritic spine heads to penetrate pre-synaptic nerve terminals (Spacek and Harris, 2004). Their diameters range from $\sim 10$ to $\sim 150 \mathrm{~nm}$ and their lengths from $\sim 30$ to $\sim 750 \mathrm{~nm}$. Spinules are often surrounded by coated pits on the interior of the membrane of the pre-synaptic terminal, contain few organelles, receive few synapses, and, like spine mats, can be trans-endocytosed into the presynaptic terminal (Spacek and Harris, 2004). Although spinules can follow a developmental profile of expression in cerebellar mossy terminals (Eckenhoff and Pysh, 1979; Tao-Cheng et al., 2009; Zancan et al., 2015), in their dimension and lack of innervation, they differ from type 1 and 2 spines.

Dendritic spines. Dendritic spines are short protrusions from the dendritic shaft and are sites for excitatory synaptic transmission at the spine head and, to a lesser extent, along the spine neck (Araya, 2014; Bartol et al., 2015). The spine neck can be thin (0.1 - $0.4 \mu \mathrm{m}$ diameter, 


\section{Chapter 3}

hippocampus) relative to the spine head ( $1 \mu \mathrm{m}$ for mushroom spines (Bourne and Harris, 2008)). Typical spine lengths are 1-3 $\mu \mathrm{m}$ (Bartol et al., 2015; Gipson and Olive, 2017), and in cortex averaged $1.8 \mu \mathrm{m}$ (maximum length $3.8 \mu \mathrm{m}$ ) and $0.1 \mu \mathrm{m}^{3}$ in volume (Kasthuri et al., 2015). Somatic type 1 spines were shorter on average, but lengths and volumes fell within the ranges described for dendritic spines (Suppl. Fig. 1). The geometry of the spine neck may isolate synapses electrically from other sites on the dendrite (Noguchi et al., 2005; Araya et al., 2006) however, type 1 spines in the MNTB lack a clear neck or head, and their diameters (mean: $0.32 \pm$ $0.09 \mu \mathrm{m})$ were somewhat larger than the dendritic spine neck. Therefore, the simple morphology of type 1 spines lacks the functional specialization to isolate currents from the soma, which is typically modelled to be nearly isopotential with the spike initiation zone. Type 1 spines were innervated mostly at their base, but also along their length and at their tip, and type 2 spines along their lateral membranes underneath the $\mathrm{CH}$. Due to the general similarities in geometry and innervation between dendritic spines and type 1 somatic spines, and in contrast to the smaller caliber and lack of innervation in spinules, we favored naming these somatic processes as type 1 somatic spines.

Thorny execresences and toric spines. A comparable structure to the somatic type 2 spines is the thorny excrescence that forms along dendrites of CA3 pyramidal neurons. These branched spines cluster on the proximal dendrite of CA3 pyramidal cells, embed into mossy fiber terminals of dentate gyrus granule cells without glial intervention (Chicurel and Harris, 1992; Popov et al., 2011), and, like type 2 spines, begin to form after expansion of the presynaptic terminal (Amaral and Dent, 1981) These structures resemble type 2 spines in that they are clustered and branched, but they are not intertwined. Moreover, each branch of the process is innervated, and their entire 


\section{Chapter 3}

surfaces are tightly apposed to the mossy fiber pre-synaptic membrane. Unlike type 2 spines, thorny excrescences are a feature of mature neurons. Recently, complex spines called toric spines, because some have looped components, have been described on the soma and basal dendrites of space-specific inferior colliculus neurons in the barn owl (Sanculi et al., 2020). Unlike type 2 spines, toric spines were unbranched, had larger volume, and were innervated by multiple different inputs via up to tens of synapses. The spine mats underlying the $\mathrm{CH}$ share many features with spine mats in the embryonic and adult avian ciliary ganglion, which have complex geometry, are intertwined, and can be contacted by synapses (Shoop et al., 2002). The constituent processes of these spine mats were named somatic spines by Shoop et al. (2002) and, in keeping with that precedent terminology, we also name these structures spine mats and somatic spines, but distinguish the individual components as a separate type (type 2) from somatic spines that occur singly (type 1).

\section{Potential functions of type 1 spines and spine mats}

Developmental profile. The increased density of somatic spines until P9, irrespective of the size of the $\mathrm{CH}$ nerve terminal (Fig. 6), and lack of correlation with the relative size of competing terminals (Fig. 7), suggests that emergence of spines and spine mats reflects maturation of the postsynaptic principal neuron. An initial phase of physiological maturation, in which, among other properties, membrane input resistance decreases and spike threshold increases (Hoffpauir et al., 2010; Rusu and Borst, 2011), is concurrent with increased type 1 spine density, which adds membrane surface area and directly contributes to these functional measures. We cannot know the outcome of competition from static images produced by SBEM (smaller CHs with higher spine density grow into a dominant $\mathrm{CH}$ ), and the correlation of density with size at P4 is 
intriguing, but the lack of a consistent relationship at P3 and P6 precludes drawing firm conclusions. As spine mat density peaks between P6 and P9, the principal cell is reorganizing its axonal field to a narrower range of frequency laminae in the LSO (Kim and Kandler, 2003; Clause et al., 2014). These elements of downstream principal cell maturation may be communicated, as another element of coordinated pre- and postsynaptic maturation (Hoffpauir et al., 2010), via type 2 spine interaction with the maturing calyx and extending to local astrocytes via their contribution of processes to the spine mat.

Intercellular communication. A notable feature of the growing $\mathrm{CH}$ is its adherence to the postsynaptic membrane over large areas of membrane without intervening extracellular space or glial incursion (Sätzler et al., 2002; Hoffpauir et al., 2006). These membrane apposition areas are potential sites for intercellular communication via adhesion proteins, clustered into adhering junctions as most clearly revealed by transmission EM (Sätzler et al., 2002; Hoffpauir et al., 2006) or distributed along the membrane surface. We did not observe presynaptic membrane invaginations without type 1 spines, so the two structural elements form cooperatively and may also impart mechanical stability to the growing $\mathrm{CH}$ in an environment of rapid structural dynamics.

Both type 1 and type 2 spines have roles in intercellular chemical communication since they are sites for innervation. The postsynaptic composition of AMPA receptor subunits shifts during development (P7-21) toward incorporation of more GluA3 and GluA4 subunits for faster, high fidelity transmission (Joshi et al., 2004; Koike-Tani et al., 2005). Moreover, application of a selective open channel blocker of calcium permeable AMPARs revealed immature principal 


\section{Chapter 3}

cells (P4-5), and "maturing" (P14) cells, receive a relatively higher $\mathrm{Ca}^{2+}$ influx than mature principal cell synapses (P30) (Joshi et al., 2004; Lujan et al., 2019). The decline in type 1 and type 2 spine density after P9 may contribute to a reduction in synaptic sites and an alteration in AMPAR composition although it is tempting to speculate calcium permeable AMPARs drive $\mathrm{Ca}^{2+}$ signaling locally at spines beneath developing CHs. This first description of organelle exclusion within type 1 spines and extending into the underlying somatic cytoplasm merits further study using high resolution electron tomography and other super-resolution approaches to ascertain the prevalence of cytoskeletal elements, such as actin, that may provide clues to the function and dynamics of this cellular region. Type 2 spines, in contrast to type 1 spines, contain organelle structures and can be tortuous and very thin at many locations along their length, so the substrates may exist for multiple modes of communication from electrically and chemically isolated compartments. These signaling modes may mediate synaptic plasticity and local processing within the spine mat that communicates among the intertwined neuronal and glial processes as glial and neuronal elements transform structurally into a mature circuit (RodríguezContreras et al., 2008; Holcomb et al., 2013; Dinh et al., 2014; Saliu et al., 2014; Brandebura et al., 2018).

Spine mats are not a prevalent feature of P30 CHs. Spine mat number could be reduced by each cell withdrawing its processes, or the structural elements could be engulfed. SBEM images that capture (1) spine mats comprised of attached and detached structures, where the fraction of detached structures ranges from near zero to near 100 percent, (2) detached structures in partial states of engulfment including those appearing to be in the final stage of pinching off, and (3) fully detached intertwined processes enclosed within the $\mathrm{CH}$, provide strong support for a 


\section{Chapter 3}

mechanism of their removal via trans-endocytosis. This evidence is like that in support of transendocytosis of spinules that extend from dendritic spines in hippocampus into their associated presynaptic terminals (Spacek and Harris, 2004). Trans-endocytosis may serve both to regulate the number of spine mats, but also to move signaling molecules from glial and postsynaptic cells into the presynaptic terminal. We noted also a layer around the engulfed structure that excludes other cytoplasmic organelles (Suppl. Fig. 2). This direct exchange of cellular material between cell types may ensure tightly coordinated maturation through the second postnatal week when the ear canal opens demarcating the onset of hearing (Ehret, 1976; Romand and Ehret, 1990; Kamiya et al., 2001; Sonntag et al., 2009), structural refinement of the $\mathrm{CH}$ via fenestration, and tuning of synaptic transmission for more precise and faster kinetics (Taschenberger and von Gersdorff, 2000; Ford et al., 2009; Takahashi, 2015).

Future work will require high-resolution light microscopy methods to visualize type 1 and 2 spines along with associated presynaptic and glial membranes to, ideally, track their dynamics in vitro or in vivo. High resolution electron tomography, conducted over sufficiently large volume, would also shed light on the intra and intercellular elements of spines and the associated localized regions of the postsynaptic cell body. 


\section{Chapter 4}

\section{Chapter 4: Role of Spontaneous Activity on the Growth of the CH}

Introduction:

Many nuclei of the central auditory circuit initially begin to assemble during later embryonic and early postnatal development in rodents (Gurung and Fritzsch, 2004; Clause et al., 2014). For example, stimulation of the auditory nerve fiber can elicit the first central responses in cells of the ventral cochlear nucleus (VCN) at E15 (Marrs and Spirou, 2012). During the same period of time (E14-15), globular bushy cell (GBC) axons are actively navigating across the midline towards contralateral targets including the principal neurons of the medial nucleus of the trapezoid (MNTB) (Howell et al., 2007). GBC axons that form terminal arbors within the MNTB will have established the first functional contacts with principal cells between E17-18, just ahead of birth (Marrs and Spirou, 2012). Beginning between postnatal (P) days P2-3, a limited number of the GBC bouton-type terminals begin to expand such that each principal cell (PC) is contacted by at least a single protocalyx, and some may be contacted by 2 or 3 enlarged terminals (Hoffpauir et al., 2006; Holcomb et al., 2013). Ultimately, competition resolves such that each principal cell is contacted by one dominant input between P6-9 that will persist and mature into the calyx of Held (CH) (Morest, 1968a; Kandler and Friauf, 1993; Bergsman et al., 2004; Holcomb et al., 2013).

Throughout the period between initial GBC axon extension (Howell et al., 2007) and establishment of the CH (P6-9) (Bergsman et al., 2004; Holcomb et al., 2013), regulated axonal endo- and exocytosis is vital to the secretion of and response to both signaling and guidance molecules during axon outgrowth as well as neurotransmitter release for transsynaptic communication (Itofusa and Kamiguchi, 2011; Tojima and Kamiguchi, 2015). Moreover, 


\section{Chapter 4}

supporting cells of the neonatal cochlea secrete ATP that triggers spontaneous activity in hair cells (Tritsch et al., 2007) that percolate as spontaneous bursts of activity across multiple nuclei throughout the auditory brainstem of pre-hearing adolescent rodents (Tritsch and Bergles, 2010; Tritsch et al., 2010). Although activity-dependent mechanisms have also been speculated to influence the selection of "winning" inputs (and removal of others) from sites of competition necessary for tonotopic refinement in the auditory system (Sanes and Takács, 1993; Friauf and Lohmann, 1999), the exact mechanism that leads to the establishment of a single $\mathrm{CH}$ is still largely unknown (Yu and Goodrich, 2014).

Traditional methods for eliminating activity in the developing auditory system generally involved lesioning one or both cochlea (Kitzes et al., 1995; Russell and Moore, 1995; Franklin et al., 2008). The limitation therein is the difficulty in separating the effect of cell necrosis following chronic deinnervation of cells and or trauma in the cochlear nucleus from the lack of presynaptic neurotransmission. Genetic manipulations of hair cells have targeted the mechanotransduction channel, $\mathrm{Ca} 2+$ channels, and the vesicular glutamate transporter, in order to reduce or eliminate synaptic transmission onto spiral ganglion neurons. In these animals, no effect on CH formation was noted (Youssoufian, 2005; Noh et al., 2010; Hirtz et al., 2011). The effectiveness of these manipulations on spiral ganglion cell spike activity was not directly assayed. In the case that spiral ganglion neurons are mostly or completely silenced by these manipulations, a possibility remains that GBCs homeostatically alter their spike thresholds to generate spontaneous activity.

In order to inhibit action potential (AP) evoked release of neurotransmitter specifically from developing protocalyceal terminals, we took advantage of a conditional mouse line that expresses a GFP-tox fusion construct, RC::PFtox (Kim et al., 2009). Tox desensitizes the readily 


\section{Chapter 4}

releasable pool to Ca2+ influx via tetanus toxin light chain's (TeTxLC) ability to proteolytically cleave the synaptic vesicle-associated membrane protein (VAMP2) and inhibit assembly of the SNARE complex needed for association and fusion with the plasma membrane (Schiavo et al., 2000). Conditional expression of GFP-tox in in the developing neonatal population of GBCs prevented the formation of large cup-shaped terminals at up to $40 \%$ of principal cells $(\mathrm{p}=0.00164$, z-test), and a similar proportion produced irregularly-shaped terminals that cover significantly smaller proportions of the respective somatic surface ( $p=1.269 \mathrm{E}-07, \mathrm{z}$-test $)$. We also employed a secondary strategy targeting GBCs through expression of a tox construct (HdAd28E4 Cpun TeTxLC syn mCherry) delivered into newborn mice. Expression of the viral cassette occurred quickly in transfected cells (within 3 days), reducing fusion competency during the period of initial terminal expansion and preventing evoked release event (Holcomb et al., 2013).

Methods:

Ethical Approval. All procedures utilizing animals were performed with approval from the Institutional Animal Care and Use Committee of West Virginia University in accordance with the United States Public Health Service and the United States National Institutes of Health guidelines.

Animals. Selective conditional expression of the fusion protein GFP-Tox was achieved by breeding RC::PFtox homozygous female mice (a generous gift from Susan Dymecki (Kim et al., 2009) to male FLPo recombinase mice (Jackson Laboratory; 129 ROSA26Flpo). Resulting

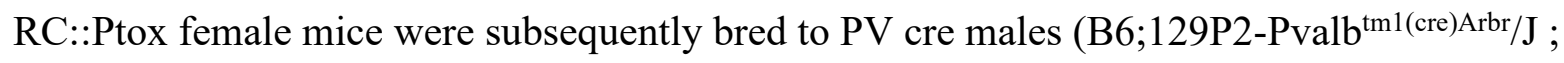
Jackson Laboratory, Stock No. 008069). 


\section{Chapter 4}

Virus Injection. P0 RC::PFtox mice (identified as day of birth) were temporarily removed from their mother and deeply anesthetized via hypothermia for a period of six minutes. Pups were then placed onto a pre-chilled, clay-filled aluminum block, with the head oriented into approximately the horizontal plane. Coordinates for lambda and bregma were determined using an injection needle lowered near the skull and held in a Kopf stereotaxic frame (model 940 digital; David Kopf Instruments, Tujunga, CA). Before introducing the virus, a small (1-2 mm) incision was produced across the scalp with a separate $26 \mathrm{G}$ needle to expose the skull. The following injection coordinates were used (in $\mathrm{mm}$ ) relative to bregma in order to target the (VCN): medial-lateral, 1.4; anterior-posterior, 5.1; dorsal-ventral, 3.2-2.9. The injection needle was filled with a solution containing a recombinant adenovirus (rAd) optimized for fast, highlevel expression of both the fluorescent reporter mCherry as well as the $50 \mathrm{kDa}$ light chain of tetanus toxin, a metalloprotease that cleaves the vesicle-associated membrane protein synaptobrevin (VAMP) driven by the efficient pUNISHER cassette (Montesinos et al., 2011). A final volume of $0.5 \mu \mathrm{L}\left(0.33 \mu \mathrm{L}\right.$ of HdAd28E4 Cpun TeTxLC syn mCherry at $4.41 \times 10^{8}$ viral particles $/ \mu \mathrm{L}$ stock mixed with $0.17 \mu \mathrm{L}$ of $20 \%$ mannitol in storage buffer containing $10 \mathrm{mM}$ HEPES, 250mM sucrose, and $1 \mathrm{mM} \mathrm{MgCl}_{2} ; \mathrm{pH}$ 7.4) was injected using a calibrated injection capillary (Blaubrand; intraMARK, Wertheim, Germany) at a rate of $0.1 \mu \mathrm{L} / \mathrm{min}$. Once the solution was injected, the glass needle was kept in place for an additional 4 min to allow the dissipation of backpressure before slow removal from the skull. Animals were then placed under a heat lamp $\left(\sim 35^{\circ} \mathrm{C}\right)$ to recover. Pups were ultimately returned to their mothers and monitored to ensure pups began to nurse.

Immunohistochemistry. Postnatal mice of either sex were anesthetized with an IP injection of Tribromoethanol (Avertin, $250 \mathrm{mg} / \mathrm{Kg}$ ). Animals were perfused first with $15 \mathrm{~mL}$ of 


\section{Chapter 4}

normal Ringer's solution (room temperature) followed subsequently by $30 \mathrm{~mL}$ of phosphate buffered saline containing 4\% paraformaldehyde (PFA; room temperature; Fisher Scientific, Pittsburgh, PA). The brain was removed from the skull and post-fixed overnight in chilled $4 \%$ PFA $\left(4^{\circ} \mathrm{C}\right)$. Brains were next transferred to cryoprotectant $(30 \%$ sucrose in $0.075 \mathrm{M}$ sodium phosphate buffer, $\mathrm{pH} 7.4 ; 4^{\circ} \mathrm{C}$ ) for a second overnight incubation. Coronal sections of the brainstem were sliced to a thickness of $40 \mu \mathrm{m}$ using a freezing microtome (model HM450, Microm, Waltham, MA). Free floating sections containing the MNTB were transferred to blocking solution (3\% goat serum in PBS containing 0.1\% Triton X-100) for a period of $1 \mathrm{hr}$. Then, tissue was incubated overnight at $4^{\circ} \mathrm{C}$ with gentle agitation in a solution containing the following primary antibodies diluted in 3\% goat serum in PBS: chicken polyclonal anti-MAP2 (1:2,500, Encor Biotechnology Inc.); guinea pig polyclonal anti-VGluT 1 and 2 (1:2500; EMD Millipore); rabbit polyclonal anti-GFP (1:500, Molecular Probes); chicken polyclonal antineurofilament (1:10,000, EMD Millipore).

The following day, sections were repeatedly rinsed in PBS. Secondary antibodies diluted (1:500; Molecular Probes) in 3\% blocking solution were applied to sections and incubated for two hours at room temperature with gentle agitation. Sections then undergo a final series of washes in PBS before free floating sections are transferred to a FluoroDish (WPI) cell culture dish and mounted on an inverted an inverted Zeiss LSM 710 confocal microscope equipped with a motorized stage (Zeiss Microscopy).

Segmentation and three-dimensional reconstruction. Principal cells and their associated inputs contained within confocal image volumes were extracted using Seg3D software (Scientific Computing and Imaging Institute, University of Utah). A total of 287 cells were analyzed using the following pipeline: The thresholding tool in Seg3D was used mask the 


\section{Chapter 4}

highest density of signal at each channel. Manual inspection and editing of the resulting masks produced reconstructions of the volume of each terminal as well as cell body following the manual cleavage of neurites. Masks in the VGluT channel were expanded uniformly by a single pixel to produce a temporary mask layer. The Boolean AND tool was used to merge the segmentation of the soma with the temporary "expanded calyx" mask to produce a complex twodimensional sheet representing the area of apposition of the terminal. All subsequent quantification calculations were performed in Microsoft Excel (Microsoft, Redmond, WA).

Statistics. All data are presented as mean \pm standard deviation or as relative within group proportion. Statistical significance across multiple groups was evaluated with a Kruskal-Wallis Test with Nemenyi post-hoc test with a Bonferroni correction to control for the number of groups. The difference between group proportions were compared with the multiple-proportion z-test.

Results:

\section{Constitutive GFP-tox expression in the developing auditory brainstem.}

In a previous study taking advantage of a novel "whole-head" preparation that maintained parts of the peripheral cochlea within a sectioned piece of brain tissue, we have revealed several events in $\mathrm{CH}$ development that occur during late embryonic ages (Marrs and Spirou, 2012). These included the observation that stimulation of spiral ganglion neurons elicited synaptic responses in the VCN beginning at E15 (Marrs and Spirou, 2012) and that it takes at most two additional days for efferent bushy cell projections to become functional in the MTNB by E17 (Hoffpauir et al., 2010). With the goal of blocking these early synaptic events specifically between bushy cells and MNTB neurons, we employed a transgenic mouse model reliant on two 


\section{Chapter 4}

recombination events for conditional expression of a tetanus toxin fusion protein construct

(Figure 1) (Kim et al., 2009). These mice are referred to as RC::PFtox mice. In the presence of Cre recombinase, the mice express an mCherry reporter. Breeding these mice to PV-Cre resulted in relatively restricted mCherry expression in the auditory brainstem as evidenced by clear labeling of cells within the VCN, including bushy cells (compare A and B in Figure 1), as well as within the axonal projections of the VCN along the ventral acoustic stria (VAS) to targets in the lateral superior olive (LSO) and medial nucleus of the trapezoid body (MNTB), among others (Figure 1 D). However, note that Flp-mediated recombination is still required for expression of the GFP-tox construct (Figure 1 C).

Surprisingly, the penetrance of the RC::PFtox was variable despite confirmation of appropriate allele expression. Our first litter of RC::tox pups yielded several pups with the same genotype (PV-cre, FLPo, RC::tox) but which exhibited this variable penetrance. Two pups had a severe motor ataxia evident by the end of the first postnatal week ('strong phenotype') while two littermates of the same genotype exhibited no motor impairment ('weak phenotype'). For ethical reasons and concerns affected that pups may have difficulty feeding, this litter was sacrificed at P9 and the MNTB was collected alongside an age-matched control litter of RC::PFtox mice. In addition to genetically expressed fluorescence, immunolabeling was performed for Vglut1 and 2, markers for the $\mathrm{CH}, \mathrm{MAP} 2$, a marker for the MNTB cell body, and neurofilament-2 (NF2), a marker for axons. The CHs identified in control tissue looked typical for this age, with nearly all principal cells (87\%) supporting a single large cup-like terminal, in close agreement with estimates of $\mathrm{CH}$ growth and maturation reported previously $(88 \%$ resolved to monoinnervation by P9; Holcomb et al, 2013)(blue and green channels, respectively, in Figure 2A). RC::tox pups exhibited disrupted terminal formation. In the less penetrant example (Figure $2 \mathrm{~B}$ ), $\sim 50 \%$ of the 


\section{Chapter 4}

imaged principal cells were contacted by large presynaptic terminals relative to RC::PFtox controls, and nearly $\sim 40 \%$ were not contacted by a large terminal ( $\mathrm{p}=0.00164$, z-test; Figure 2D). The organization of the local innervation varied further in ataxic triple transgenic pups wherein $\sim 25 \%$ of principal cells were not contacted by a large terminal (Figure $2 \mathrm{C}, \mathrm{D})$. Many $(\sim 40 \%)$ of the large terminals that formed appeared atypically swollen and blebbed (red and blue channels). Only $14 \%$ of principal cells were contacted by a normal appearing terminal $(p=1.269 \mathrm{E}-07, \mathrm{z}-$ test; Figure 2D).

Heterogeneous tox expression in the developing auditory brainstem.

Constitutive expression of GFP-tox in some animals disrupted normal structural maturation of the calyx of Held onto many MNTB neurons. We previously investigated the early stages of calyx formation through a series of serial electron micrograph volumes collected from mice across the first postnatal weeks of life (including P2, P3, P4, P6, P9, P30) in order to chart the morphological features exhibited during terminal expansion. We demonstrated a transient period of synaptic competition (Holcomb et al., 2013; Jackson et al., 2020). As an alternative to variable penetrance of genetic manipulation, we next sought a more consistent method to silence GBC synaptic activity through viral transfection of a TeTx construct. This manipulation was designed to determine whether reduced synaptic activity would preclude the growth of a calyx terminal. Pups were injected on the day of birth (P0) with a viral vector (HdAd28E4 Cpun TeTxLC syn mCherry) that was delivered focally within the left VCN (Figure 3A). By P3, trace amounts of mCherry expression were detectable in the VCN (not shown). By P5, robust labeling of cells in the injected VCN (Figure 3B-C) was observed complete with efferent projections 


\section{Chapter 4}

along the ventral acoustic stria that entered the lateral superior olive (LSO) bilaterally, (Figure 3B) and the contralateral MNTB (Figure 3F). The injection into one $\mathrm{CN}$ transfected additional neurons in the contralateral VCN (Figure 3D), although these cells did not produce CHs within the ipsilateral MNTB (relative to the injection site; Figure 3E). Details of the injection process remain to be worked out so that the contralateral projection can be used as a within-animal control group.

Tiled z-stacks collected bilaterally from each MNTB were imaged at higher magnification to quantify these observations. No virally labeled (mCherry) CHs were identified within the ipsilateral MNTB although commonly large terminals were defined by clusters of VGLUT staining (two curved profiles in Figure 4A). Within the contralateral MNTB, relative to the injection site, we found a combination of mCherry positive (arrowheads) and mCherry negative (blue) terminals (Figure 4B). These tiled image volumes from each nucleus were then exported to Seg3D where channel data was independently thresholded and masked to produce an approximate reconstruction of each pre- and postsynaptic compartment. A combination of manual inspection and smoothing was then used to refine these 3D renderings into individual pre- and postsynaptic compartment reconstructions as well as define a planar border representing the area of apposition between the terminal and the postsynaptic PC soma. (note* In order to study primarily the effect of the synaptic silencing through constitutive TeTxLC expression, only principal cells that were contacted by at least one mCherry positive profile were analyzed in the “contralateral group”).

Quantification of the partial reconstructions produced from the previous experiment suggested a role for neural activity in $\mathrm{CH}$ growth and maturation. Focusing first on PCs that were contacted by only a single calyceal terminal, mCherry/TeTxLC expressing terminals in the 


\section{Chapter 4}

contralateral MNTB exhibited an average thickness of $1.1 \pm 1.01 \mu \mathrm{m}$, which was nearly double the values relative to $\mathrm{CHs}$ within the ipsilateral MNTB $(0.49 \pm 0.42 \mu \mathrm{m}$ thick $(\mathrm{p}=2.77 \mathrm{E}-8, \mathrm{KW}$; Figure 4C)). We also quantified the size of the terminal by measuring its apposed surface area (ASA) and overall volume. Ipsilateral (non virally-transduced) $\mathrm{CH}$ terminals exhibited significantly larger ASAs than mCherry/TeTxLC positive terminals in the contralateral MNTB (349.69 $\pm 268.56 \mu \mathrm{m}^{2}$ ipsilateral vs $188.02 \pm 130.50 \mu \mathrm{m}^{2}$ contralateral; $p=4.33 \mathrm{E}-3, \mathrm{KW}$; Figure 4D). Despite differing thickness and ASA, the overall volumes of the terminals were similar between groups $\left(206.94 \pm 249.35 \mu \mathrm{m}^{3}\right.$ ipsilateral vs $215.98 \pm 209.44 \mu \mathrm{m}^{3}$ contralateral; $\mathrm{p}=$ 0.403, KW; Figure 4E). Finally, we noticed a nearly $25 \%$ reduction in the somatic volume of the postsynaptic principal neurons that receive innervation from $\mathrm{mCherry}$ positive $\mathrm{CHs}$ in the contralateral MNTB $\left(1615.26 \pm 444.78 \mu \mathrm{m}^{3}\right.$ ipsilateral vs $1208.59 \pm 312.48 \mu \mathrm{m}^{3}$ contralateral; $\mathrm{p}=2.63 \mathrm{E}-6, \mathrm{KW}$; Figure 4F).

Finally, the competitive outcomes of all MNTB PCs both ipsilateral and contralateral to the injection site were assessed similarly to the previous experiment. However, NF labeling additionally allowed the axons of each potential input to be differentially tracked within the volume. Counts of the number of unique inputs to each cell are summarized in Table 1. Most dramatic at P5 was the $\sim 30 \%$ reduction in the number of PCs that host a single dominant $\mathrm{CH}(\mathrm{p}=$ 0.00289 , z-score). Moreover, over $40 \%$ of the principal cells imaged within the contralateral MNTB hosted two or more large terminals with unique axons.

Discussion: 


\section{Chapter 4}

Use of the so-called "toxigenetic" model, or selective genetic expression of tetanus toxin light chain (TeTxLC), within subpopulations of neurons allows for direct manipulation of patterns of activity in living and developing neural circuits. Expression of the $50 \mathrm{kDa}$ metalloprotease TeTxLC inhibits neurotransmitter release through proteolytic cleavage of the synaptic vesicleassociated membrane protein (VAMP2) (Schiavo et al., 2000). In a number of studies, perturbation in VAMP2 expression through the introduction of TeTxLC or genetic deletion (syb2 $\mathrm{KO}$ ) results in a 50-90\% reduction in action potential evoked events, although some basal level of spontaneous vesicular fusion still occurs (Schiavo et al., 2000; Schoch et al., 2001; Yamamoto et al., 2003). However, even in the context of a abolishment of synaptic communication via a conditional muc-13 deletion and subsequent deficit in synaptic vesicle priming capacity, synaptogenesis has still been shown to occur (Varoqueaux et al., 2002). So, it is perhaps unsurprising, despite expression of TeTxLC, GBC axons were capable of producing large terminals.

Silenced axons in this dataset exhibited a significant disruption in terminal growth over the first postnatal week. We found through conditional expression of GFP-tox across the population of GBCs produced a variable phenotype that could result in up to a $40 \%$ reduction in the number of principal cells contacted by a large terminal. Moreover, many of the terminals that persisted appeared swollen and irregular. Previous studies investigating the ultrastructural characteristics of cells that have chronically expressed tox have found synaptic vesicles can aggregate as a result of disrupted exocytosis leading to an engorgement of presynaptic boutons (Kim et al., 2009). It is likely a similar phenomenon occurred in our mutated GBC efferents as revealed by the near complete overlap of VGLUT and GFP labeling in immunohistochemical 


\section{Chapter 4}

preparations. However, it is currently unclear why the penetrance of the phenotype in RC::tox animals varied within litters and poses challenges as a reproducible model.

In order to verify the phenotype observed in transgenic animals, we additionally utilized viral transfection as a secondary method of TeTxLC expression. Particular interest during quantification of histological preparations was paid to principal cells that were contacted by at least one silenced input or no inputs at all. Although unreported, a larger number of principal cells were surveyed in order to meet this criterion as a result. Nonetheless, viral-mediated silencing of a proportion of GBCs still resulted in a similar reduction in the number of principal cells that failed to be contacted by a large terminal (30\% vs 40\%). Among cells that supported at least a single mCherry positive input, there was a $30 \%$ increase in the number of polyinnervated principal cells (42\% contralateral vs $13 \%$ ipsilateral). This could be suggestive of a persistence of competing inputs when both competitors have similar levels of activity. Similar studies at the neuromuscular junction have raised similar possibilities. Blocking all AChR at a developing motor endplate with an irreversible antagonist, such as $\alpha$-bungarotoxin, or genetically inhibiting the generation of an action potential through the overexpression of inwardly rectifying potassium channels post-synaptically in mice have each been shown to inhibit normal synapse elimination (Balice-Gordon and Lichtman, 1994; Favero et al., 2009). On the opposite end of the spectrum, driving synchronous activity through all competing inputs also inhibited synapse elimination at individual muscle fibers in rats (Busetto et al., 2000). However, artificially enhancing the disparity in firing strength and frequency between competing axons lead to an immediate loss of the less active input (Keller-Peck et al., 2001). These data helped to establish an understanding that the relative imbalance of activity among competing inputs could serve as an activity- 


\section{Chapter 4}

dependent trigger for permissive growth of a single input or instructive for the removal of others (Kasthuri and Lichtman, 2003; Walsh and Lichtman, 2003).

Moving forward, the toxigenetic model offers a unique platform to model the role of synaptic activity in the development of neural circuits. Although large terminals could form despite significant perturbation to the exocytic capacity of these terminals, additional experiments are necessary in order to draw firm conclusions on the persistence of polyinnervation as a result of interrupted activity-dependent pruning. Our quantification relied on incomplete fluorescent staining that could only approximate changes in gross morphology and axonal identity. Moreover, verification and quantification of the persistence of spontaneous release events too will further our understanding of the effectiveness of our silenced model. 


\section{Chapter 5}

\section{Chapter 5: Discussion and Future Directions}

In many parts of the developing nervous system, more synaptic connections are initially formed than exist at maturity. Activity-dependent refinement of initial connections via selective pruning is a necessary process in order to increase the precision of circuit connectivity. In the case of sensory systems wherein this phenomena is perhaps most well-studied, the resulting arrangement of highly precise connectivity between brain regions is referred to as topographic organization (Udin and Fawcett, 1988). In neocortex and other brain regions, many synapses, or functional connections between neurons, are formed at the interface of a presynaptic bouton and a dendritic spine head (Berry and Nedivi, 2017). Bouton terminals typically contain one or two active zones. However, very large inputs are located in a limited number of brain regions (see NMJ, CF:PC, RGC:dLGN; and CH:MNTB described in Chapter 1), and can contain hundreds to over one thousand distinct vesicle release sites, which ensures release of multiple synaptic vesicles and increased reliability of synaptic transmission (Walmsley et al., 1998; von Gersdorff and Borst, 2002; Rudolph et al., 2015). Examples such as the $\mathrm{CH}$, the largest nerve terminal in the mammalian central nervous system, offer key advantages in the study of neural development due to the exaggerated size and relative simplicity of the mature endpoint of 1:1 innervation ratio of the $\mathrm{CH}$ and MNTB principal neuron. The results described in Chapters 2, 3 and 4 provide meaningful new insights into the structural and functional changes that underlie the growth and maturation of this largest central nerve terminal. The major conclusions of those studies will now be discussed in the broader context of neural circuit formation including a brief discussion of remaining questions and presentation of future research directions. 


\section{Chapter 5}

Elaboration $\mathrm{CH}$ collateral arborization

Studies of the fine structure of the developing $\mathrm{CH}$ have occurred over the last few decades, yet little attention was paid to the collateral processes that emanate from the edges of the $\mathrm{CH}$ (Morest, 1968a; Kandler and Friauf, 1993). Building upon this work, the Spirou laboratory developed the first and largest collection of serial electron micrograph volumes of tissue collected from the medial, high-frequency MNTB of mice, taken at various early postnatal ages (Holcomb et al., 2013). Accurate, high-resolution reconstructions of both presynaptic terminals and associated principal neurons had to be painstakingly obtained by hand. However, these efforts charted the structural expansion of multiple large terminals that initially grew over each principal neuron at younger ages (P2-4) as well as the emergence of a single dominant input just days later (P6 \& 9) (Hoffpauir et al., 2006; Holcomb et al., 2013). These 3D reconstructions, in parallel with physiologic measurements taken from similar ages, both indicate that principal cells are contacted briefly by multiple large protocalyceal terminals (Hoffpauir et al., 2006, 2010). Calycigenic axons also have numerous branches that may support transient synaptic activity (Kuwabara et al., 1991; Kandler and Friauf, 1993; Rodríguez-Contreras et al., 2006; Holcomb et al., 2013). In rare cases, the calycigenic axon branches and delivers two CHs within close proximity, typically within $50 \mu \mathrm{m}$, such that tonotopic precision is maintained (Kuwabara et al., 1991; Rodríguez-Contreras et al., 2006).

We proposed and other experiments support a transient period of synaptic competition that resolves to monoinnervation (Holcomb et al., 2013; Xiao et al., 2013; Milinkeviciute et al., 2019). We hypothesized that competition resulted from indirect competition between competing inputs as each growing $\mathrm{CH}$ established an apposed surface area (ASA) along distinct, nonoverlapping regions of the MNTB cell somatic surface (Holcomb et al., 2013). However, 


\section{Chapter 5}

consideration of collateral growth of the $\mathrm{CH}$ requires reconsideration of whether more direct signaling occurs directly between competing inputs. More recently, the dynamics of axon branch and perhaps $\mathrm{CH}$ collaterals were observed in vivo, (Rodríguez-Contreras et al., 2008). In our most recent efforts, we sought to address fundamental questions about the emergence, function and motility of $\mathrm{CH}$ collaterals, using lattice light sheet (LLS) dynamic imaging in combination with a further analysis using our SBEM image series.

We assessed whether collaterals are a feature of later stages of $\mathrm{CH}$ growth, once the $\mathrm{CH}$ has anchored a position on the postsynaptic cell body, or are extended as soon as the protocalyx, the first expansion of the small terminal, begins to form. At P3 about one-half of MNTB cells in the SBEM volume are contacted by growing terminals of at least the protocalyx stage $\left(\sim 35 \mu \mathrm{m}^{2}\right)$, and about one-half (43\%) of these cells are contacted by two or three large terminals (Holcomb et al., 2013). Therefore, we focused on these smallest terminals, and noted that they already extended collaterals of each type: short filopodia, long filopodia, long growth cone tipped collaterals. One important question is why would a terminal expend the requisite resources and energy to produce an extensive network of collateral arborization network that will largely be removed within days? We considered that these extensions could provide either or both of exploratory roles, seeking out additional territories for innervation, or defensive roles in preventing competition from other calycigenic axons. Notably both potential functions are underway at the protocalyx stage due to the presence of all collateral types.

The length of $\mathrm{CH}$ collaterals places a limit on their sampled volume. Nearly all long collaterals are $60 \mu \mathrm{m}$ or less, with a few growth cone-tipped collaterals reaching $100 \mu \mathrm{m}$ or longer. Except for the longest collaterals, the exploratory volume could be within the tonotopic medial-lateral specificity of the MNTB (total distance $\sim 350 \mu \mathrm{m}$ medial-lateral at early postnatal 


\section{Chapter 5}

ages in mice (Kolson et al., 2016; Weatherstone et al., 2016), especially since collaterals had a range of orientations and thus were typically not aligned along the frequency axis. Interestingly, protocalyces at P3 had already extended long collaterals, and the increase in number of collaterals with $\mathrm{CH}$ size did not alter the distribution of collateral lengths, but did add additional long collaterals which presumably increased the patrolled volume by these structures. Initial exuberance and overconnectivity of presynaptic afferents has been a well-described phenomena in a neurodevelopmental context in a variety of brain regions (Innocenti and Price, 2005; Portera-Cailliau et al., 2005; Gallo, 2011). Thus, if longer CH collaterals were capable of forming synaptic contacts, then this system could serve as a fail-safe to ensure that each PC is innervated and each GBC axon delivers at least one $\mathrm{CH}$ into the MNTB. A constraining endpoint of the system is that each calycigenic axon produces primarly one $(90 \%)$ or occasionally two $(10 \%) \mathrm{CH}$ terminals, and in the latter case maintains tonotopic organization (Spirou et al., 1990; Kuwabara et al., 1991; Smith et al., 1991; Rodríguez-Contreras et al., 2006). Furthermore, approximately $10 \%$ of MNTB neurons are contacted by two CHs at P9, an arrangement that may persist into adulthood.

SBEM permitted us to evaluate the structures that were contacted by long $\mathrm{CH}$ collaterals. Nearly $20 \%$ of long growth cone tipped collaterals, which constituted 124/634 long collaterals, were found to physically contact multiple principal cells, which are densely packed, along the length of the collateral and occasionally at their tips. Careful inspection of SBEM images revealed no evidence of synaptic contacts along $\mathrm{CH}$ collaterals. However, immature synapses can form transiently even without the full complement of synaptic proteins (Lee and Peng, 2008). We did observe what appeared to be synaptic vesicles within long filopodial and growth cone tipped $\mathrm{CH}$ collaterals. Together with observed $\mathrm{Ca}^{2+}$ signals along the length of calycigenic 


\section{Chapter 5}

axon branches (Rodríguez-Contreras et al., 2008), we suggest that $\mathrm{CH}$ collaterals may provide transient innervation to multiple principal cells. Growth cone tipped processes are linked to synaptogenesis in other systems (Chilton, 2006; Gomez and Letourneau, 2014). Notably, at least a subset of mature $\mathrm{CHs}$ ( 1 in 6 at P30) maintain a single collateral branch onto a second nearby principal cell via a smaller terminal. Thus, we hypothesize an exploratory role for long $\mathrm{CH}$ collaterals to identify additional innervation targets, in case a growing $\mathrm{CH}$ loses competition onto its initial cell target and must form a new $\mathrm{CH}$ onto another cell, is associated with one of the few GBC axons forming two $\mathrm{CHs}$, or is establishing a functional $\mathrm{CH}$ collateral that persists in the mature circuit.

SBEM permitted more detailed views into $\mathrm{CH}$ collaterals than afforded by previous light microscopy studies and revealed that $50 \%$ of collaterals were $\sim 6 \mu \mathrm{m}$ in length or shorter (median length: $5.85 \pm 9.32 \mu \mathrm{m}, \mathrm{n}=1,352$ of 2970 collaterals, $\mathrm{n}=34 \mathrm{CHs})$. These collaterals were filopodial in morphology, mostly devoid of internal structures, and extended laterally from the edge of the $\mathrm{CH}$ without contacting the somatic surface and mostly terminate within the extracellular space without direct contact with adjacent cells. In static images, we were unable to ascertain if short collaterals could lengthen to become more complex. However, given the relative constancy of the distribution of collateral length between P3-6, we propose that a least a subpopulation remain short. Such structures may establish a static perimeter that could provide a leading edge to $\mathrm{CH}$ growth, or subsequently attach to the cell body surface as part of $\mathrm{CH}$ expansion.

We next investigated whether $\mathrm{CH}$ collaterals contacted other $\mathrm{CH}$ collaterals, either from the same or from different cells. These contacts could represent a substrate for preserving innervation territory and perhaps mediating competition onto the same MNTB cell. Long 


\section{Chapter 5}

collaterals were often branched; and collateral branches were typically filopodial in shape. In SBEM images, we found nearly half $(55.7 \%)$ of long collaterals make physical contact with at least one additional collateral from a nearby $\mathrm{CH}$ or a nearby principal neuron (Chapter 5 Figure 1). In static images, however, we were unable to assess the functional dynamics that resulted from these contacts. Given the rapid estimated growth rates for the $\mathrm{CH}$ during the early postnatal period (Holcomb et al., 2013), a steady fraction of short collaterals should be become long to maintain the length distribution across $\mathrm{CH}$ size.

\section{Dynamic Motility and Potential Function of CH Collaterals}

Our next steps towards evaluating the behavior of $\mathrm{CH}$ collaterals involved direct observation of collateral motility using the LLS microscope. This microscope achieves diffraction limited resolution in all three axes (Langhorst et al., 2009) with the low power presentation of an ultrathin plane of illumination produced by dithering a custom array of nonGaussian Bessel beams (Chen et al., 2014). We developed a preparation that allowed a fresh section of perinatal tissue to be mounted within an oxygenated perfusion chamber for an acute imaging session. The rapid volumetric image acquisition rate varied between experiments but recorded collateral dynamics at a maximum frequency approaching $1 \mathrm{~Hz}$. This approach eclipsed the sampling rate achieved in the only previous study of dynamic motility of filopodial processes in the developing MNTB (Rodríguez-Contreras et al., 2008). That preparation uniquely permitted in vivo imaging through a ventral cranial window; but the temporal resolution of the data was limited by a relatively low acquisition rate of 2-photon raster imaging. In that study, changes in collateral length were observed at minutes to tens of minutes intervals, and only a subset of processes were labeled, so interactions among neurites could not be monitored. 


\section{Chapter 5}

Notably, LLS imaging revealed more rapid rates of process motility within axon terminal zones than has previously been observed (Rodríguez-Contreras et al., 2008). We also noted clear differences in the types of motile behavior observed among collateral subtypes, and addressed several open questions from observations on the static EM images. We were able to image short collaterals, but due to the lower resolution of LLS relative to our ultrastructural reconstructions, we did not detect as many as were found using SBEM. Nonetheless, dynamic imaging of these structures revealed, as we predicted, that short filopodia typically did not extend to become long collaterals. We proposed that short filopodia provided a fixed structure at the edge of the $\mathrm{CH}$, but in fact these short filopodia could have short lifespans and in some cases persisted for as little as 5 minutes (11/21 short filopodia). We did not observe them interacting with long collaterals, and our sample did not include closely located regions of competing $\mathrm{CHs}$ onto the same cell. From these data we conclude that short collaterals may be more associated with expansion at the edges of the $\mathrm{CH}$ rather than playing a protective function, but additional imaging sessions may reveal interactions between different $\mathrm{CHs}$.

Long collaterals, both filopodia and growth cone tipped, exhibit a variety of movement patterns. Extension could be directed as a series of rapid linear extensions with short pauses, or collaterals could frequently change direction due to either juxtracrine interaction among labeled and unlabeled elements in the tissue or tracking of chemical gradients. Rapid extension was faster than any reports in the literature on developing nervous system, consistent with the speed with which the nervous system is assembled. These rates rarely surpass the proposed limiting rate of cytosolic and cytoskeletal extension, defined as slow axonal transport $(1 \mu \mathrm{m} / \mathrm{min})$ (Brown, 2014). Typically, it is thought that long distance migrations of axons through along fiber bundles

are the only times axons achieve these speeds, such as occurs as axons extend through the corpus 


\section{Chapter 5}

callosum and cross the midline $(1.3 \mu \mathrm{m} / \mathrm{min})$ (Halloran and Kalil, 1994). However, within targeted brain regions, axonal arborization dynamics within terminals territories rarely too can achieve impressive rates of motility. Retinotectal axons within target territories may elongate at impressive rates that exceed slow axonal transport $(5 \mu \mathrm{m} / \mathrm{min})($ Witte et al., 1996). However, in our experiments, long collaterals exhibited the fastest rates of extension. $\mathrm{CH}$ collateral growth cones achieved average values of motility of $2.1 \mu \mathrm{m} / \mathrm{min}$ during periods in which they transiently exhibit instantaneous growth rates exceeding $10 \mu \mathrm{m} / \mathrm{min}$.

In a few instances we had a sufficient number of labeled processes and a favorable field of view to identify direct contact between long filopodia from different $\mathrm{CHs}$. The most protracted interaction involved one filopodial collateral advancing upon and contacting another, withdrawing and re-contacting several times. At the end of this sequence, the advancing collateral retracted, underwent a unique windmill motion and extended in a different direction (Chapter 2, Figure 6). It is tempting to speculate that surface-mediated contact between these collaterals indicates a generalized repulsive signaling between CHs. Similar findings of repulsive axo-axon signaling via surface expression of adhesion molecules (such as Down-syndrome cell adhesion molecule 2, Dscam2; Ig superfamily protein, Turtle) have been implicated in the developmental axonal segregation of ocular dominance columns of the medulla mediated directly though axo-axonal contact (Millard et al., 2007; Ferguson et al., 2009). Effectively, these descriptions of repulsive interactions represent the initial evidence for direct signaling between growing $\mathrm{CHs}$ in the developing MNTB. Additional experiments will be required to more fully elucidate the valence of long collateral interactions and explore the molecular mechanisms that underlie collateral behaviors. 


\section{Chapter 5}

Postsynaptic specializations during growth of the $\mathrm{CH}$

Despite a rich literature representing over a century of study investigating the shape and function of dendritic spines as sites for presynaptic innervation (Yuste and Bonhoeffer, 2004; Yuste, 2015), new structures are continuing to be discovered that broaden our understanding of the potential shape and function of postsynaptic spines (Sanculi et al., 2020). As noted in the previous section, little attention has been paid to the investigation of $\mathrm{CH}$ collaterals in the MNTB, but even less is known about the specialized somatic spines that have been observed in developing and mature tissue. We identified two types of somatic spines, which exhibit similarities in morphology to dendritic spines in their overall size (Harris et al., 1992), but differ by having larger caliber typically without an expanded head or constricted neck (type 1 somatic spines) or by being longer and branched (type 2 somatic spines). Type 1 spines were solitary and were entirely bound within invaginated pockets embedded into the presynaptic face of the overlying nerve terminal. Moreover, this arrangement ensured tight association with the presynaptic membrane along the entire surface of the type 1 spine, which provided additional sites for mechanical stability (Rowland et al., 2000) and synaptic innervation. The second type of spine, referred to as type 2 spines, exhibited a more complex morphology revealed by a complexity analysis reported in Chapter 3, but more importantly could be distinguished as a unique component in an intertwined tangle of spines that collectively reside within a shared invagination in the presynaptic face of the overlying terminal. We thus provide the first description spine mats in the CNS, as they have only been described previously at the somatic surface of ciliary ganglion neurons in the peripheral nervous system of the chick. Those neurons also are innervated by a calyx-type nerve terminal (Shoop et al., 2002). 


\section{Chapter 5}

Population dynamics and the persistence of $\mathrm{CH}$ collaterals and somatic spines

MNTB neurons are characteristically smooth at P2, prior to growth of large terminals onto their cell bodies (Holcomb et al., 2013). The first signs of $\mathrm{CH}$ growth at P3 are accompanied by a coordinated emergence of $\mathrm{CH}$ collaterals. We thus investigated in SBEM images whether the smallest protocalyces were characterized by extension of type 1 spines into the presynaptic face of the growing terminal. Recall that spine mats were present only when $\mathrm{CHs}$ exceeded about $100 \mu \mathrm{m}^{2}$ of apposed surface area, so we did not investigate their presence in this context. In only the two smallest protocalyces in our sample was there an absence of type 1 spines (input A: $35 \mu \mathrm{m}^{2}$; input B: $38.2 \mu \mathrm{m}^{2}$ ). Thus, $\mathrm{CH}$ growth can likely initiate without type 1 spine formation, but the two events quickly become coordinated. These events imply early signaling between the growing $\mathrm{CH}$ and localized regions of the postsynaptic membrane.

Both $\mathrm{CH}$ collaterals and somatic spines increased in density until P9, one day ahead of the opening of the ear canal and the onset of experience-dependent activity. After P9, calyx collaterals, nearly all type 2 spines and hence spine mats, and the vast majority of type 1 spines are removed as the $\mathrm{CH}$ achieves its mature synaptic properties and fenestrated morphology (von Gersdorff and Borst, 2002; Ford et al., 2009). A general pattern of coordination among these events seems evident, but closer examination reveals temporal differences in the expression of these pre and postsynaptic structures.

The appearance of type 1 spines and $\mathrm{CH}$ collaterals appears highly synchronized. Type 1 spine density reaches its maximum at $\mathrm{P} 6$, as does the number of $\mathrm{CH}$ collaterals exhibiting the full representation of long filopodial and growth cone tipped structures. Type 1 spine density is maintained at $\mathrm{P9}$, as is the number of $\mathrm{CH}$ collaterals, but long collaterals are few in number. P9 


\section{Chapter 5}

is the age at which we estimate the mature composition of mono and multi-innervated MNTB neurons is achieved. Spine mat density begins to increase one day after type 1 spine density and peaks at P9. $\mathrm{CH}$ collaterals, except a single thick branch from a minority of $\mathrm{CHs}$ that innervates a neighboring MNTB cell, and spine mats are nearly absent at P30, and type 1 spines are at very low density. Thus, the full complement $\mathrm{CH}$ collateral lengths are most directly tied to $\mathrm{CH}$ growth and establishment of the final CH:MNTB neural circuit. Type 1 spine expression tracks this same period, but persists into the next phase of $\mathrm{CH}$ fenestration. Spine mat expression peaks at the beginning of the phase of $\mathrm{CH}$ fenestration. We propose that $\mathrm{CH}$ collaterals play roles in synaptic competition, but we did not find differentiating features among competing $\mathrm{CHs}$ to suggest a similar role for type 1 spines and spine mats. $\mathrm{CH}$ collaterals and type 1 spines may be initiated by the same signaling processes between pre and postsynaptic cells, and these events may have downstream triggers for the formation of spine mats. Presumably, these molecular and cellular cues for process formation are active only during this narrow time period and are no longer required in the mature circuit. An accompanying schematic reveals the relative timing of each of these events (Chapter 5, Figure 1).

Importantly, we found little evidence of type 1 spines and no examples of spine mats outside of areas associated with presynaptic innervation. Thus, they are likely specialized specifically for communication with the presynaptic terminal. Transmission EM studies have revealed the $\mathrm{CH}: \mathrm{MNTB}$ junction incorporates hundreds of discrete release sites and adherens junctions during development (Sätzler et al., 2002; Hoffpauir et al., 2006; Holcomb et al., 2013). Postsynaptic densities and adherens junctions are not reliably revealed by en bloc staining for SBEM, so in particular the presence of adherens junctions could not be assessed along the direct apposition between type 1 spines and the presynaptic membrane. Nonetheless, we suggest that in 


\section{Chapter 5}

addition to providing mechanical stability, adherens junctions and regions of direct membrane apposition as around type 1 spines may also serve a communicative function via surface contactmediated interaction via transmembrane adhesion receptors. These are primarily comprised of cadherins, protocadherins, L1-CAM in the MNTB, but may also include nectins and nectin-like families of proteins that are found in many parts of the nervous system (Niessen and Gottardi, 2008; Indra et al., 2013; Kolson et al., 2016). The large diversity and non-uniform expression of adhering proteins expressed within the MNTB may be sufficient to differentiate targets across the nucleus for tonotopic organization (Whitlon et al., 1999).

In addition to contact-mediated signaling, direct signal exchange may also be mediated through connexin 36-associated gap junctions shown to be expressed using light microscopy at the synaptic interface of the $\mathrm{CH}$ and PC (Rubio and Nagy, 2015). However, our SBEM image volumes were not of sufficiently high resolution to reveal these structures. Future experiments utilizing immuno-EM or peroxidase labeling against connexin 36 in SBEM tissue blocks can reveal additional information about specialized signaling through somatic spines in the developing MNTB.

Signaling capacity of somatic spines

Before considering signaling through somatic spines, it is worthwhile to briefly consider the sequence of events that leads to their appearance. Notably, the formation of type 1 spines is synchronized to initiation of $\mathrm{CH}$ growth. Our hypothesis, to be tested in future experiments, is that emergence of bursting activity is the trigger, and if true that must induce molecular signals that reorganize membrane and cytoskeletal elements that constitute the spines. Some leads can be gleaned from the literature on dendritic spines, but the role for neural activity remains unclear 


\section{Chapter 5}

(Yuste and Bonhoeffer, 2004; Freire et al., 2010). In cerebellum, for example, despite loss of granule cells and their axons in Weaver mutant mice, Purkinje cells grow dendrites studded with spines. This phenotype suggests that intrinsic programs can promote the emergence of spines (Rakic and Sidman, 1973; O’Brien and Unwin, 2006), although climbing fiber activation could stimulate spine formation. It is not surprising that inducing reorganization of the actin cytoskeleton is essential to building spines, and has been shown via manipulations in the level of expression of the small GTPases Rac and Rho (Yuste and Bonhoeffer, 2004; Woolfrey and Srivastava, 2016). Overexpression of Rac1 is sufficient to produce shortened but persistent, supernumerary spines on Purkinje cell dendrites (Luo et al., 1996) and expression of a dominantnegative Rac-1 protein led to the progressive loss of spines (Nakayama et al., 2000). RhoA overexpression, in contrast, produced smoothened dendrites, an effect mediated by the activation of the downstream kinase ROCK (Nakayama et al., 2000). As the CH expands over the MNTB somatic surface, local contact via membrane proteins such as adhesion molecules, or neural activity, could induce type 1 spine formation. Future manipulations of $\mathrm{CH}$ activity should include an anatomical survey for the presence of type 1 spines.

When considering a functional role for the emergence of somatic spines between the developing $\mathrm{CH}$ and MNTB cell, we propose three modes of communication via somatic spines: (1) chemical synaptic transmission, (2) adhesion molecules at apposed locations of the pre and postsynaptic cells, and (3) trans-endocytosis of spine mats. The GBC to MNTB synapse is glutamatergic at least from E17, the earliest age that it has been monitored (Hoffpauir et al. 2006). Several studies have indicated the presence of NMDA receptors at early stages of CH growth, which indicates roles for activity induced postsynaptic $\mathrm{Ca}^{2+}$-signaling to mediate 


\section{Chapter 5}

competition and maintenance of synaptic contact (Taschenberger and von Gersdorff, 2000; Futai et al., 2001; Hoffpauir et al., 2006). We consider these topics further in the next sections.

Role of chemical neurotransmission in the establishment of the $\mathrm{CH}$. A variety of manipulations have been implemented previously to disrupt neurotransmission in the developing auditory system via the brute force approach of unilateral or bilateral cochlear ablation (Kitzes et al., 1995; Russell and Moore, 1995; Franklin et al., 2008) which produces significant cell loss in the cochlear nucleus, or via genetic perturbation of inner hair cells. The latter has been accomplished via Otoferlin knock-out (Roux et al., 2006; Longo-Guess et al., 2007), Cav1.3 KO (Brandt et al., 2003; Erazo-Fischer et al., 2007), VGLUT3 KO (Ruel et al., 2008; Seal et al., 2008) or knock-out of the $\alpha 9$ subunit of nicotinic acetylcholine receptors (Clause et al., 2014). However, manipulation of inner hair cells leaves open the possibility of homeostatic upregulation of spontaneous activity in spiral ganglion neurons or GBCs. Thus, the small effect of these manipulations on $\mathrm{CH}$ growth does not have clear interpretation. As described in preliminary experiments of Chapter 4, through selective expression of TeTxLC directly in GBCs, synaptic vesicle release was presumably inhibited. Despite the retained capacity for terminals to form, less pruning occurred in these TeTxLC-positive animals such that supernumerary terminals were maintained more consistently than in TeTxLC-negative controls and their geometry was altered. In order to more fully validate this phenotype, paired pre and postsynaptic voltage clamp experiments will be required to characterize the level of intrinsic spontaneous activity that persists, as well as to characterize the biophysical maturation of deprived MNTB neurons.

The maturation of the principal cells is synchronized with the growth of the $\mathrm{CH}$. For example, increases in the synaptic strength of terminals (as a byproduct of insertion of additional 


\section{Chapter 5}

release sites) is correlated highly with an increase in postsynaptic surface area and expression of low threshold potassium channels, which reduces intrinsic excitability of the neuron (Hoffpauir et al., 2010; Van Hoeve et al., 2010; Sierksma et al., 2017). Our preliminary data suggests that expression of TeTxLC presynaptically prevents the increase in somatic volume that occurs in control animals. A smaller cell size should result in a more excitable cell due to higher input resistance, which would be an appropriate homeostatic response to reduced synaptic input. Further electrophysiologic experiments will be required to more formally characterize the proposed delay in postsynaptic maturation due to abolishment of spontaneous and evoked activity. The altered morphology of the $\mathrm{CH}$ following expression of TeTX should, in our future experiments, be studied along with characterization of $\mathrm{CH}$ collaterals and somatic spine formation as one strategy to assay the primacy of physiological signaling to induce the growth and maturation of the $\mathrm{CH}: \mathrm{MNTB}$ connection.

Spine mats offer a complex geometry for segregation of signaling routes among cells, as well as extensions from presynaptic and glial compartments wherein tri-synaptic relationships can form (Müller et al., 2009; Reyes-Haro et al., 2010). The complex extracellular space, perhaps aided by diffusion barriers due to adhering junctions, may facilitate aggregation of signaling molecules secreted locally within the available extracellular space. The frequent observation of reticulated membrane within type 2 spines further suggests signaling capacity that is similar to that associated with the spine apparatus within mushroom shaped dendritic spines (Deller et al., 2003). $\mathrm{Ca}^{2+}$ signaling at this site could affect the volume of spines and weights (Jedlicka et al., 2008; Holbro et al., 2009) of the few chemical synapses onto the outer surface of the spine mat. In the only other known occurrence of spine mats, diffusion of acetylcholine (Ach) through extracellular space to bind to receptors along the component somatic spines was proposed as a 


\section{Chapter 5}

mechanism for extra-synaptic neuromodulation (Shoop et al., 1999). Gene expression analysis in MNTB (Kolson et al., 2016) revealed temporal increase of was Chrnna4 (cholinergic receptor, nicotinic, alpha polypeptide 4), message at P6 relative to P0, and subsequent decrease at P14. Cholinergic signaling affects large somatic terminal formation in the ventral nucleus of the lateral lemniscus (Baumann and Koch, 2017), so it may generally be important for the establishment of large, calyx-type terminals in the auditory brainstem.

Signaling through adhesion proteins. Signaling via adhesion molecules is a broad topic (Aplin et al., 1999; Cavallaro and Dejana, 2011). Work from our lab has identified cadherins, including N-cadherin, protocadherins as possible substrates within the MNTB (Kolson et al., 2016). These associations may form directly between CHs and type 1 spines, but also between MNTB cells and astrocyte processes within spine mats. A recent report demonstrated evidence for a novel process of nectin-mediated material exchange, whereby nectin- 1 expressing neurons trans-endocytose segments of membrane from cells containing nectin-4 (Generous et al., 2019). Thus, experimental manipulations in the expression of nectin-1 may provide potential opportunities to disrupt spine mat trans-endocytosis during the development of the MNTB.

Signaling via trans-endocytosis of spine mats. This mode of intercellular signaling offers multiple categories of molecular signals, but is more difficult to clarify, as spine mat elements contain membrane, and likely membrane bound receptors, cytoskeletal elements, diffusible cytoplasmic constituents including proteins, and organelles (Spacek and Harris, 2004; Petralia et al., 2015). Any and all of these cellular elements may provide a communication route between these synaptic partners. The onset of trans-endocytosis in the $\mathrm{CH}: \mathrm{MNTB}$ system is at P9, just 


\section{Chapter 5}

after the MNTB neuron has refined its projection territory to the lateral superior olive (LSO) (Kandler et al., 2009; Clause et al., 2014), and may represent a retrograde signal of those downstream maturation events. Trans-endocytosis at sites of synaptic communication was first described as the extension of thin elements called spinules that extended from dendritic spines and were engulfed by the presynaptic terminal (Spacek and Harris, 2004; Petralia et al., 2015). Recent studies using super-resolution light microscopy have demonstrated trans-endocytosis to be prevalent, occurring at nearly $25 \%$ of synaptic sites in layer 4 of the primary visual cortex (Campbell et al., 2020). Labeling for an early endosomal marker, like the small GTPase Rab5, may reveal evidence for a transition towards spine mat trans-endocytosis beginning between P6 and P9 while Rab7 may label fully detached spine mats (Rink et al., 2005). Our images suggest that lysosomal structures may serve as the final destination for trans-endocytosed materials. However, additional experiments will need to be performed to more carefully characterize this process.

Clues to molecular signals carried by trans-endocytosed structures may be gleaned from structures that can be similar in caliber $(100 \mathrm{~nm})$ but longer $(50-100 \mu \mathrm{m})$, called cytonemes. First described in the wing imaginal disc cells in Drosophila, cytonemes are specialized filopodia that transport signaling proteins directly between cells (Ramírez-Weber and Kornberg, 1999). Cytonemes have been described in both neuronal (Casas-Tintó and Portela, 2019) and nonneuronal cell types (Kornberg and Roy, 2014a, 2014b) but in all cases exist as thin, actinsupported filopodia that are responsible for juxtacrine signaling. Cytoneme subtypes can be defined by their response to different attractant signals, such as the Drosophila homolog of the vertebrate bone morphogenic protein (BMP), Decapentaplegic (Dpp); or the epidermal growth factor protein, Spitz (Roy et al., 2012). Once guided to appropriate signaling targets, cytonemes 


\section{Chapter 5}

may exert their effect through surface expression of membrane bound proteins. For example, hedgehog $(\mathrm{Hh})$ proteins, enriched along cytonemes protruding from support cap cells, are secreted locally onto nearby niche cells (Rojas-Ríos et al., 2012). Newly delivered Hh protein activates the downstream transcription of multiple bone morphogenic genes including Dpp and Glass bottom boat $(\mathrm{Gbb})$ in escort cells and provides a mechanism directly regulate local stem cell proliferation (Rojas-Ríos et al., 2012). Alternatively, cytonemes may in other cases deliver membrane bound molecules via trans-endocytosis (Parker et al., 2017). Finally, cytonemes are also capable of forming "gap junction-like" pores when contacting a second cell, producing a cytoneme variant called a tunneling nanotube. Tunneling nanotubes have been shown to bridge cells up to $100 \mu \mathrm{m}$ apart, and were responsible for direct trans-endocytic exchange of cytoplasmic material between nervous cells (Vidulescu et al., 2004). Moreover, these cytoneme variants create web-like linkages between cells that can be stabilized and maintained in neural circuits and have been implicated in the pathophysiologic transfer of both amyloid A $\beta$ peptide and tau during the progressive development of degenerative Alzheimer's disease (Wang et al., 2011; Tardivel et al., 2016). We do not have direct evidence for this type of intercellular communication, but our SBEM images were not of sufficiently high resolution to capture even transient expression of patent intercellular bridges. Future work in the MNTB will need to establish the molecular composition of the membrane-bound constituents of somatic spines, and provide very high resolution images of the intercellular border, in order to investigate cytonemelike function in the development of the $\mathrm{CH}: \mathrm{MNTB}$. 


\section{Chapter 5}

General conclusions

The establishment of the CH:MNTB functional connection has emerged as a model system to study neural circuit formation. The large size of the $\mathrm{CH}$ and its rapid emergence, the relatively simple to assay endpoint of 1:1 connectivity, and stereotypical stages of development allow researchers to ask very fundamental questions about the mechanisms that give rise to a mature neural connection. The ultrastructural information revealed here through the perspective of our time series of SBEM image volumes and lattice light sheet dynamic imaging affords a unique opportunity to directly observe novel aspects of tissue reorganization that constitute neural circuit development. For example, we have characterized previously described single somatic spines and $\mathrm{CH}$ collaterals, provided clues to the dynamics and function of $\mathrm{CH}$ collaterals, and added a new element, the spine mat, to the unfolding story of neural circuit formation. The synchronized onset of pre and postsynaptic structural changes, and differential temporal profiles of the developmental events that we describe here, offer a baseline to study their underlying mechanistic signals. We hypothesize that the trigger is an altered pattern of spontaneous activity. Future experiments linking physiological and molecular perturbation with high resolution light microscopy will provide necessary platform to extract a more complete description of the sequence of structural plasticity characteristic of the formation of the CH:MNTB connection. We propose that lattice light sheet imaging with new combinations of cellular labels and molecular perturbation can achieve these goals. Although the $\mathrm{CH}$ system is among the few that achieve mono or few:one innervation ratios, insights gathered will likely apply to those other systems and aspects of $\mathrm{CH}$ development will likely have generalizable relevance to neural circuits formation throughout the CNS. 


\section{References:}

Ackman JB, Burbridge TJ, Crair MC (2012) Retinal waves coordinate patterned activity throughout the developing visual system. Nature 490:219-225.

Ahrens MB, Orger MB, Robson DN, Li JM, Keller PJ (2013) Whole-brain functional imaging at cellular resolution using light-sheet microscopy. Nat Methods 10:413-420.

Alvarado-Kristensson M, Rodríguez MJ, Silió V, Valpuesta JM, Carrera AC (2009) SADB phosphorylation of gamma-tubulin regulates centrosome duplication. Nat Cell Biol 11:1081-1092.

Amaral DG, Dent JA (1981) Development of the mossy fibers of the dentate gyrus: I. A light and electron microscopic study of the mossy fibers and their expansions. J Comp Neurol 195:51-86.

Andjus P., Zhu L, Cesa R, Carulli D, Strata P (2003) A change in the pattern of activity affects the developmental regression of the purkinje cell polyinnervation by climbing fibers in the rat cerebellum. Neuroscience 121:563-572.

Aplin AE, Howe AK, Juliano RL (1999) Cell adhesion molecules, signal transduction and cell growth. Curr Opin Cell Biol 11:737-744.

Araya R (2014) Input transformation by dendritic spines of pyramidal neurons. Front Neuroanat $8: 1-18$.

Araya R, Jiang J, Eisenthal KB, Yuste R (2006) The spine neck filters membrane potentials. Proc Natl Acad Sci U S A 103:17961-17966.

Athamneh AIM, He Y, Lamoureux P, Fix L, Suter DM, Miller KE (2017) Neurite elongation is highly correlated with bulk forward translocation of microtubules. Sci Rep 7:1-13.

Azevedo FAC, Carvalho LRB, Grinberg LT, Farfel JM, Ferretti REL, Leite REP, Filho WJ, Lent R, Herculano-Houzel S (2009) Equal numbers of neuronal and nonneuronal cells make the human brain an isometrically scaled-up primate brain. J Comp Neurol 513:532-541.

Baas PW, Deitch JS, Black MM, Banker GA (1988) Polarity orientation of microtubules in hippocampla neurons: Uniformity in the axon and nonuniformity in the dendrite. PNAS $85: 8335-8339$.

Bak M, Fraser SE (2003) Axon fasciculation and differences in midline kinetics between pioneer and follower axons within commissural fascicles. Development 130:4999-5008.

Balice-Gordon RJ, Lichtman JW (1993) In vivo observations of pre- and postsynaptic changes during the transition from multiple to single innervation at developing neuromuscular junctions. J Neurosci 13:834-855.

Balice-Gordon RJ, Lichtman JW (1994) Long-term synapse loss inuced by focal blockade of postsynaptic receptors. Nature 372:519-524.

Ball R, Xing B, Bonner P, Shearer J, Cooper RL (2003) Long-term in vitro maintenance of neuromuscular junction activity of Drosophila larvae. Comp Biochem Physiol - A Mol Integr Physiol 134:247-255.

Barnes AP, Polleux F (2009) Establishment of axon-dendrite polarity in developing neurons. Annu Rev Neurosci 32:347-381.

Bartol TM, Bromer C, Kinney J, Chirillo MA, Bourne JN, Harris KM, Sejnowski TJ (2015) Nanoconnectomic upper bound on the variability of synaptic plasticity. Elife 4:1-18.

Baumann VJ, Koch U (2017) Perinatal nicotine exposure impairs the maturation of glutamatergic inputs in the auditory brainstem. J Physiol 595:3573-3590.

Bellar JA, Kulengowski B, Kobraei EM, Curinga G, Calulot CM, Bahrami A, Hering TM, Snow 
DM (2013) Comparison of sensory neuron growth cone and filopodial responses to structurally diverse aggrecan variants, in vitro. Exp Neurol 247:143-157.

Bergsman JB, De Camilli P, McCormick DA (2004) Multiple large inputs to principal cells in the mouse medial nucleus of the trapezoid body. J Neurophysiol 92:545-552.

Berry KP, Nedivi E (2017) Spine dynamics: Are they all the same? Neuron 96:43-55.

Beutner D, Moser T (2001) The presynaptic function of mouse cochlear inner hair cells during development of hearing. J Neurosci 21:4593-4599.

Bi G, Poo M (2001) Synaptic modification by correlated activity: Hebb's postulate revisited. Annu Rev Neurosci 24:139-166.

Bickford ME, Slusarczyk A, Dilger EK, Krahe TE, Kucuk C, Guido W (2010) Synaptic development of the mouse dorsal lateral geniculate nucleus. J Comp Neurol 518:622-635.

Bilimoria PM, Bonni A (2014) Molecular control of axon branching. Neuoscientist 71:38313840.

Biroc SL, Payan DG, Fisher JM (1993) Isoforms of agrin are widely expressed in the developing rat and may function as protease inhibitors. Dev Brain Res 75:119-129.

Bishop DL, Misgeld T, Walsh MK, Gan W-B, Lichtman JW (2004) Axon branch removal at developing synapses by axosome shedding. Neuron 44:651-661.

Blanchoin L, Boujemaa-Paterski R, Sykes C, Plastino J (2014) Actin dynamics, architecture, and mechanics in cell motility. Physiol Rev 94:235-263.

Blankenship AG, Feller MB (2010) Mechanisms underlying spontaneous patterned activity in developing neural circuits. Nat Rev Neurosci 11:18-29.

Bonanomi D, Chivatakarn O, Bai G, Abdesselem H, Lettieri K, Marquardt T, Pierchala BA, Pfaff SL (2012) Ret Is a multifunctional coreceptor that integrates diffusible- and contactaxon guidance signals. Cell 148:568-582.

Borst JGG, Sakmann B (1996) Calcium influx and transmitter release in a fast CNS synapse. Lett to Nat 383:431-434.

Borst JGG, Soria van Hoeve J (2012) The calyx of Held synapse: from model synapse to auditory relay. Annu Rev Physiol 74:199-224.

Bourne JN, Harris KM (2008) Balancing structure and function at hippocampal dendritic spines. Annu Rev Neurosci 31:47-67.

Brandebura AN, Morehead M, Heller DT, Holcomb P, Kolson DR, Jones G, Mathers PH, Spirou GA (2018) Glial cell expansion coincides with neural circuit formation in the developing auditory brainstem. Dev Neurobiol 78:1097-1116.

Brandt A, Striessnig J, Moser T (2003) CaV1.3 channels are essential for development and presynaptic activity of cochlear inner hair cells. J Neurosci 23:10832-10840.

Bray D, Chapman AK (1985) Analysis of microspike movements on the neuronal growth cone. J Neurosci 5:3204-3213.

Brew HM, Forsythe ID (1995) Two voltage-dependent K+ conductances with complementary functions in postsynaptic integration at a central auditory synapse. J Neurosci 15:80118022.

Brose K, Bland KS, Hong Wang K, Arnott D, Henzel W, Goodman CS, Tessier-Lavigne M, Kidd T (1999) Slit proteins bind Robo receptors and have an evolutionarily conserved role in repulsive axon guidance. Cell 96:795-806.

Brown A (2003) Axonal transport of membranous and nonmembranous cargoes: A unified perspective. J Cell Biol 160:817-821.

Brown A (2014) Slow Axonal Transport 弥. Elsevier Inc. 
Brown MC, Jansen JKS, Essen D Van (1976) Polyneuronal innervation of skeletal muscle in new-bown rats and its elimination during maturation. J Physiol 261:387-422.

Bryda EC (2014) The mighty mouse: the impact of rodents on advances in biomedical research. Mo Med 110:207-211.

Buffelli M, Burgess RW, Feng G, Lobe CG, Lichtman JW, Sanes JR (2003) Genetic evidence that relative synaptic efficacy biases the outcome of synaptic competition. Nature 424:430434.

Busch SA, Horn KP, Silver DJ (2009) Overcoming macrophage-mediated axonal dieback following CNS injury. J Neurosci 29:9967-9976.

Busetto G, Buffelli M, Tognana E, Bellico F, Cangiano A (2000) Hebbian mechanisms revealed by electrical stimulation at developing rat neuromuscular junctions. J Neurosci 20:685-695.

Caicedo A, Eybalin M (1999) Glutamate receptor phenotypes in the auditory brainstem and midbrain of the developing rat. Eur J Neurosci 11:51-74.

Campbell C, Lindhartsen S, Knyaz A, Erisir A, Nahmani M (2020) Cortical presynaptic boutons progressively engulf spinules as they mature. eNeuro 7:1-10.

Cant NB, Benson CG (2003) Parallel auditory pathways: Projection patterns of the different neuronal populations in the dorsal and ventral cochlear nuclei. Brain Res Bull 60:457-474.

Cant NB, Morest DK (1979) The bushy cells in the anteroventral cochlear nucleus of the cat. A study with the electron microscope. Neuroscience 4:1925-1945.

Carrasco MM, Trujillo M, Razak K (2013) Development of response selectivity in the mouse auditory cortex. Hear Res 296:107-120.

Casas-Tintó S, Portela M (2019) Cytonemes, their formation, regulation, and roles in signaling and communication in tumorigenesis. Int J Mol Sci 20:23-25.

Cavallaro U, Dejana E (2011) Adhesion molecule signalling: Not always a sticky business. Nat Rev Mol Cell Biol 12:189-197.

Chan SSY, Zheng H, Su MW, Wilk R, Killeen MT, Hedgecock EM, Culotti JG (1996) UNC-40, a C. elegans homolog of DCC (Deleted in Colorectal Cancer), is required in motile cells responding to UNC-6 netrin cues. Cell 87:187-195.

Chédotal A, Richards LJ (2010) Wiring the brain: the biology of neuronal guidance. Cold Spring Harb Perspect Biol 2:1-18.

Chen B-C et al. (2014) Lattice light-sheet microscopy: Imaging molecules to embryos at high spatiotemporal resolution. Science (80- ) 346.

Chen C, Regehr WG (2000) Developmental remodeling of the retinogeniculate synapse. Neuron 28:955-966.

Chen Z, Das B, Nakamura Y, DiGregorio DA, Young SM (2015) Ca2+ channel to synaptic vesicle distance accounts for the readily releasable pool kinetics at a functionally mature auditory synapse. J Neurosci 35:2083-2100.

Chen Z, Lee H, Henle SJ, Cheever TR, Ekker SC, Henley JR (2013) Primary neuron culture for nerve growth and axon guidance studies in zebrafish (Danio rerio). PLoS One 8.

Chicurel ME, Harris KM (1992) Three-dimensional analysis of the structure and composition of CA3 branched dendritic spines and their synaptic relationships with mossy fiber boutons in the rat hippocampus. J Comp Neurol 325:169-182.

Chien C Bin, Rosenthal DE, Harris WA, Holt CE (1993) Navigational errors made by growth cones without filopodia in the embryonic xenopus brain. Neuron 11:237-251.

Chilton JK (2006) Molecular mechanisms of axon guidance. Dev Biol 292:13-24.

Chowdhury S, Shepherd JD, Okuno H, Lyford G, Petralia RS, Plath N, Kuhl D, Huganir RL, 
Paul F (2006) Arc interacts with the endocytic machinery to regulate AMPA receptor trafficking. Neuron 52:445-459.

Chretien D, Metoz F, Verde F, Karsenti E, Wade RH (1992) Lattice defects in microtubules: Protofilament numbers vary within individual microtubules. J Cell Biol 117:1031-1040.

Clause A, Kim G, Sonntag M, Weisz CC, Vetter D, Rubsamen R, Kandler K (2014) The precise temporal pattern of prehearing spontaneous activity is necessary for tonotopic map refinement. Neuron 82:822-835.

Coggan JS, Bartol TM, Esquenazi E, Stiles JR, Lamont S, Martone ME, Berg DK, Ellisman MH, Sejnowski TJ (2005) Evidence for ectopic neurotransmission at a neuronal synapse. Science (80- ) 309:446-451.

Cohen-Cory S, Lom B (2004) Neurotrophic regulation of retinal ganglion cell synaptic connectivity: From axons and dendrites to synapses. Int J Dev Biol 48:947-956.

Coleman HA, Labrador J-P, Chance RK, Bashaw GJ (2010) The Adam family metalloprotease Kuzbanian regulates the cleavage of the roundabout receptor to control axon repulsion at the midline. Development 137:2417-2426.

Colman H, Nabekura J, Lichtman JW (1997) Alterations in synaptic strength preceding axon withdrawal. Science (80- ) 275:356-361.

Cramer KS, Rubel EW (2016) Glial cell contributions to auditory brainstem development. Front Neural Circuits 10:1-8.

Crins TTH, Rusu SI, Rodríguez-Contreras A, Borst JGG (2011) Developmental changes in short-term plasticity at the rat calyx of held synapse. J Neurosci 31:11706-11717.

Curfs MHJM, Gribnau AAM, Dederen PJWC, Bergervoet-Vernooij HWM (1995) Transient functional connections between the developing corticospinal tract and cervical spinal interneurons as demonstrated by c-fos immunohistochemistry. Dev Brain Res 87:214-219.

Davies AM (2008) Neurotrophins giveth and they taketh away. Nat Neurosci 11:627-628.

de Castro F, López-Mascaraque L, De Carlos JA (2007) Cajal: Lessons on brain development. Brain Res Rev 55:481-489.

De Felipe J, Marco P, Fairén A, Jones EG (1997) Inhibitory synaptogenesis in mouse somatosensory cortex. Cereb Cortex 7:619-634.

de la Torre JR, Höpker VH, Ming G, Poo M, Tessier-Lavigne M, Hemmati-Brivanlou A, Holt CE (1997) Turning of retinal growth cones in a netrin-1 gradient mediated by the netrin receptor DCC. Neuron 19:1211-1224.

Deerinck TJ, Bushong EA, Lev-Ram V, Shu X, Tsien RY, Ellisman MH (2010) Enhancing serial block-face scanning electron microscopy to enable high resolution 3-D nanohistology of cells and tissues. Microsc Microanal 16:6-7.

Deller T, Korte M, Chabanis S, Drakew A, Schwegler H, Stefani GG, Zuniga A, Schwarz K, Bonhoeffer T, Zeller R, Frotscher M, Mundel P (2003) Synaptopodin-deficient mice lack a spine apparatus and show deficits in synaptic plasticity. Proc Natl Acad Sci U S A 100:10494-10499.

Dent EW, Gupton SL, Gertler FB, Chédotal A, Richards LJ, Frank B, Adams RH, Eichmann A, Engle EC, Raper J, Mason C (2011) The growth cone cytoskeleton in axon outgrowth and guidance. Cold Spring Harb Perspect Biol 3.

Di Bonito M, Narita Y, Avallone B, Sequino L, Mancuso M, Andolfi G, Franzè AM, Puelles L, Rijli FM, Studer M (2013) Assembly of the auditory circuitry by a Hox genetic network in the mouse brainstem. PLoS Genet 9.

Dinh ML, Koppel SJ, Korn MJ, Cramer KS (2014) Distribution of glial cells in the auditory 
brainstem: Normal development and effects of unilateral lesion. Neuroscience 278:237252.

Dodson PD, Barker MC, Forsythe ID (2002) Two heteromeric Kv1 potassium channels differentially regulate action potential firing. J Neurosci 22:6953-6961.

Dogterom M, Koenderink GH (2019) Actin-microtubule crosstalk in cell biology. Nat Rev Mol Cell Biol 20:38-54.

Dotti CG, Sullivan C a, Banker G a (1988) The establishment of polarity by hippocampal neurons in culture. J Neurosci 8:1454-1468.

Drachman DA (2005) Do we have brain to spare? Neurology 64:2004-2005.

Dulcert A, Changeux J-P (1995) Acetylcholine receptor gene expression at the developing neuromuscular junction. Physiol Rev 75:339-368.

Eckenhoff MF, Pysh JJ (1979) Double-walled coated vesicle formation: evidence for massive and transient conjugate internalization of plasma membranes during cerebellar development. J Neurocytol 8:623-638.

Egea J, Klein R (2007) Bidirectional Eph-ephrin signaling during axon guidance. Trends Cell Biol 17:230-238.

Ehret G (1976) Development of absolute auditory thresholds in the house mouse (Mus musculus). J Am Audiol Soc 1:179-184.

Elezgarai I, Díez J, Puente N, Azkue JJ, Benítez R, Bilbao A, Knöpfel T, Doñate-Oliver F, Grandes P (2003) Subcellular localization of the voltage-dependent potassium channel $\mathrm{Kv} 3.1 \mathrm{~b}$ in postnatal and adult rat medial nucleus of the trapezoid body. Neuroscience 118:889-898.

Erazo-Fischer E, Striessnig J, Taschenberger H (2007) The role of physiological afferent nerve activity during in vivo maturation of the calyx of Held synapse. J Neurosci 27:1725-1737.

Farago AF, Awatramani RB, Dymecki SM (2006) Assembly of the brainstem cochlear nuclear complex is revealed by intersectional and subtractive genetic fate maps. Neuron 50:205218.

Favero M, Buffelli M, Cangiano A, Busetto G (2010) The timing of impulse activity shapes the process of synaptic competition at the neuromuscular junction. Neuroscience 167:343-353.

Favero M, Busetto G, Cangiano A (2012) Spike timing plays a key role in synapse elimination at the neuromuscular junction. Proc Natl Acad Sci 109:E1667-E1675.

Favero M, Lorenzetto E, Bidoia C, Buffelli M, Bussetto G, Cangiano A (2007) Synapse formation and elimination: role of activity studies in differnt models of adult reinnervation. J Neurosci Res 85:2352-2359.

Favero M, Massella O, Cangiano A, Buffelli M (2009) On the mechanism of action of muscle fibre activity in synapse competition and elimination at the mammalian neuromuscular junction. Eur J Neurosci 29:2327-2334.

Fazeli A, Dickinson SL, Hermiston ML, Tighe R V, Steen RG, Small CG, Stoeckli ET, KeinoMasu K, Masu M, Rayburn H, Simons J, Bronson RT, Gordon JI, Tessier-Levigne M, Weinberg RA (1997) Phenotype of mice lacking functional Deleted in colorectal cancer (DCC) gene. Nature 386:796-804.

Fedchyshyn MJ, Wang L-Y (2005) Developmental transformation of the release modality at the calyx of Held synapse. J Neurosci 25:4131-4140.

Ferguson K, Long H, Cameron S, Chang WT, Rao Y (2009) The conserved Ig superfamily member turtle mediates axonal tiling in Drosophila. J Neurosci 29:14151-14159.

Flanagan JG (2006) Neural map specification by gradients. Curr Opin Neurobiol 16:59-66. 
Flavell SW, Greenberg ME (2008) Signaling mechanisms linking neuronal activity to gene expression and plasticity of the nervous system. Annu Rev Neurosci 31:563-590.

Ford MC, Grothe B, Klug A (2009) Fenestration of the calyx of Held occurs sequentially along the tonotopic axis, is influenced by afferent activity, and facilitates glutamate clearance. J Comp Neurol 514:92-106.

Forsythe ID (1994) Direct patch recording from identified presynaptic terminals mediating glutamatergic EPSCs in the rat CNS, in vitro. J Physiol 479 ( Pt 3:381-387.

Franklin SR, Brunso-Bechtold JK, Henkel CK (2008) Bilateral cochlear ablation in postnatal rat disrupts development of banded pattern of projections from the dorsal nucleus of the lateral lemniscus to the inferior colliculus. Neuroscience 154:346-354.

Franzen DL, Gleiss SA, Berger C, Kumpfbeck FS, Ammer JJ, Felmy F (2014) Development and modulation of intrinsic membrane properties control the temporal precision of auditory brainstem neurons. J Neurophysiol:524-536.

Freire M, García-López P, García-Marín V (2010) Dendritic spines and development: Towards a unifying model of spinogenesis a present day review of cajal's histological slides and drawings. Neural Plast 2010.

Friauf E, Lohmann C (1999) Development of auditory brainstem circuitry. Activity-dependent and activity-independent processes. Cell Tissue Res 297:187-195.

Futai K, Okada M, Matsuyama K, Takahashi T (2001) High-fidelity transmission acquired via a developmental decrease in NMDA receptor expression at an auditory synapse. J Neurosci 21:3342-3349.

Gallo G (2011) The cytoskeletal and signaling mechanisms of axon collateral branching. Dev Neurobiol 71:201-220.

Garner CC, Waites CL, Ziv NE (2006) Synapse development: Still looking for the forest, still lost in the trees. Cell Tissue Res 326:249-262.

Garraghty PE, Shatz CJ, Sur M (1988) Prenatal disruption of binocular interactions creates novel lamination in the cat's lateral geniculate nucleus. Vis Neurosci 1:93-102.

Generous AR, Harrison OJ, Troyanovsky RB, Mateo M, Navaratnarajah CK, Donohue RC, Pfaller CK, Alekhina O, Sergeeva AP, Indra I, Thornburg T, Kochetkova I, Billadeau DD, Taylor MP, Troyanovsky SM, Honig B, Shapiro L, Cattaneo R (2019) Trans-endocytosis elicited by nectins transfers cytoplasmic cargo, including infectious material, between cells. J Cell Sci 132.

Georgiou M, Tear G (2002) Commissureless is required both in commissural neurones and midline cells for axon guidance across the midline. Development 129:2947-2956.

Geraldo S, Gordon-Weeks PR (2009) Cytoskeletal dynamics in growth-cone steering. J Cell Sci 122:3595-3604.

Gipson CD, Olive MF (2017) Structural and functional plasticity of dendritic spines - root or result of behavior? Genes, Brain Behav 16:101-117.

Godement P, Salaün J, Imbert M (1984) Prenatal and postnatal development of retinogeniculate and retinocollicular projections in the mouse. J Comp Neurol 230:552-575.

Gomez TM, Letourneau PC (2014) Actin dynamics in growth cone motility and navigation. J Neurochem 129:221-234.

Gonzales RB, DeLeon Galvan CJ, Rangel YM, Claiborne BJ (2001) Distribution of thorny excrescences on CA3 pyramidal neurons in the rat hippocampus. J Comp Neurol 430:357368.

Goslin K, Banker G (1989) Experimental observations on the development of polarity by 
hippocampal neurons in culture. J Cell Biol 108:1507-1516.

Grafstein B, Forman DS (1980) Intracellular transport in neurons. Physiol Rev 60:1167-1283.

Grothe B, Pecka M, McAlpine D (2010) Mechanisms of sound localization in mammals. Physiol Rev 90:983-1012.

Grubb M, Rossi F, Changeux J, Thompson I (2003) Abnormal functional organization in the dorsal lateral geniculate nucleus of mice lacking the $\beta 2$ subunit of the nicotinic acetylcholine receptor. Neuron 40:1161-1172.

Guido W (2008) Refinement of the retinogeniculate pathway. J Physiol 586:4357-4362.

Gurung B, Fritzsch B (2004) Time course of embryonic midbrain and thalamic auditory connection development in mice as revealed by carbocyanine dye tracing. J Comp Neurol 479:309-327.

Gutierrez H, Hale V a, Dolcet X, Davies A (2005) NF-kappaB signalling regulates the growth of neural processes in the developing PNS and CNS. Development 132:1713-1726.

Hahm J-O, Langdon RB, Sur M (1991) Disruption of retinogeniculat afferent segregation by antagonists to NMDA receptors. Lett to Nat 351:568-570.

Halloran MC, Kalil K (1994) Dynamic behaviors of growth cones extending in the corpus callosum of living cortical brain slices observed with video microscopy. J Neurosci 14:2161-2177.

Harris KM, Jensen FE, Tsao B (1992) Three-dimensional structure of dendritic spines and synapses in rat hippocampus (CA1) at postnatal day 15 and adult ages: Implications for the maturation of synaptic physiology and long-term potentiation. J Neurosci 12.

Harris W a, Holt CE, Bonhoeffer F (1987) Retinal axons with and without their somata, growing to and arborizing in the tectum of Xenopus embryos: a time-lapse video study of single fibres in vivo. Development 101:123-133.

Harrison RG (1910) The outgrowth of the nerve fiber as a mode of protoplasmic movement. J Exp Zool 9:787-846.

Hashimoto K, Ichikawa R, Kitamura K, Watanabe M, Kano M (2009) Translocation of a "winner" climbing fiber to the Purkinje cell dendrite and subsequent elimination of "losers" from the soma in developing cerebellum. Neuron 63:106-118.

Hashimoto K, Kano M (2003) Functional differentiation of multiple climbing fiber inputs during synapse elimination. Neuron 38:785-796.

Hashimoto K, Kano M (2005) Postnatal development and synapse elimination of climbing fiber to Purkinje cell projection in the cerebellum. Neurosci Res 53:221-228.

Hebb DO (1949) The first stage of perception: growth of the assembly BT - The Organization of Behavior. New York: John Wiley and Sons, Inc.

Heddleston JM, Chew T-L (2016) Light sheet microscopes: Novel imaging toolbox for visualizing life's processes. Int J Biochem Cell Biol 80:119-123.

Held H (1893) Die Zentrale Gehörleitung. Arch Anat Physiol Anat Abt.

Hensch TK (2004) Critical Period Regulation. Annu Rev Neurosci 27:549-579.

Hermida D, Elezgarai I, Puente N, Alonso V, Anabitarte N, Bilbao A, Doñate-Oliver F, Grandes P (2006) Developmental increase in postsynaptic alpha-amino-3-hydroxy-5-methyl-4 isoxazolepropionic acid receptor compartmentalization at the calyx of held synapse. J Comp Neurol 495:624-634.

Hermida D, Mateos JM, Elezgarai I, Puente N, Bilbao A, Bueno-López JL, Streit P, Grandes P (2010) Spatial compartmentalization of AMPA glutamate receptor subunits at the calyx of held synapse. J Comp Neurol 518:163-174. 
Hirtz JJ, Boesen M, Braun N, Deitmer JW, Kramer F, Lohr C, Müller B, Nothwang HG, Striessnig J, Löhrke S, Friauf E (2011) Cav1.3 calcium channels are required for normal development of the auditory brainstem. J Neurosci 31:8280-8294.

Hoffpauir BK, Grimes JL, Mathers PH, Spirou GA (2006) Synaptogenesis of the calyx of Held: rapid onset of function and one-to-one morphological innervation. J Neurosci 26:55115523.

Hoffpauir BK, Kolson DR, Mathers PH, Spirou GA (2010) Maturation of synaptic partners: functional phenotype and synaptic organization tuned in synchrony. J Physiol 588:43654385.

Holbro N, Grunditz A, Oertner TG (2009) Differential distribution of endoplasmic reticulum controls metabotropic signaling and plasticity at hippocampal synapses. Proc Natl Acad Sci U S A 106:15055-15060.

Holcomb PS (2018) "Polarity and Competition in the Development of the Calyx of Held Terminal in the Medial Nucleus of the Trapezoid Body in the Mouse" PhD Dissertation.

Holcomb PS, Hoffpauir BK, Hoyson MC, Jackson DR, Deerinck TJ, Marrs GS, Dehoff M, Wu J, Ellisman MH, Spirou GA (2013) Synaptic inputs compete during rapid formation of the calyx of Held: a new model system for neural development. J Neurosci 33:12954-12969.

Hong K, Hinck L, Nishiyama M, Poo M, Tessier-Lavigne M, Stein E (1999) A ligand-gated association between cytoplasmic domains of UNC5 and DCC family receptors converts netrin-induced growth cone attraction to repulsion. Cell 97:927-941.

Hong YK, Chen C (2011) Wiring and rewiring of the retinogeniculate synapse. Curr Opin Neurobiol 21:228-237.

Howell DM, Morgan WJ, Jarjour AA, Spirou GA, Berrebi AS, Kennedy TE, Mathers PH (2007) Molecular guidance cues necessary for axon pathfinding from the ventral cochlear nucleus. J Comp Neurol 504:533-549.

Hua JY, Smith SJ (2004) Neural activity and the dynamics of central nervous system development. Nat Neurosci 7:327-332.

Hubel DH, Wiesel TN (1965) Binocular interaction in striate cortex of kittens reared with artificial squint. J Neurophysiol 28:1041-1059.

Hubel DH, Wiesel TN, LeVay S (1977) Plasticity of ocular dominance columns in monkey striate cortex. Philos Trans R Soc B Biol Sci 278:377-409.

Huber AB, Kolodkin AL, Ginty DD, Cloutier J-F (2003) Signalling at the growth cone : Ligandreceptor complexes and the control of axon growth and guidance. Annu Rev Neurosci 26:509-563.

Huttenlocher PR (1979) Synaptic density in human frontal cortex - Developmental changes and effects of aging. Brain Res 163:195-205.

Huttenlocher PR, de Courten C (1987) The development of synapses in striate cortex of man. Hum Neurobiol 6:1-9.

Imai T, Yamazaki T, Kobayakawa R, Kobayakawa K, Abe T, Suzuki M, Sakano H (2009) Pretarget axon sorting establishes the neural map topography. Science 325:585-590.

Indra I, Hong S, Troyanovsky R, Kormos B, Troyanovsky S (2013) The adhereens junction: A mosaic of cadherin and nectin clusters bundled by actic filaments. J Invest Dermatol 133:2546-2554.

Innocenti G, Fiore L, Caminiti R (1977) Exuberant projection into the corpus callosum from the visual cortex of newborn cats. Neurosci Lett 4:237-242.

Innocenti GM, Price DJ (2005) Exuberance in the development of cortical networks. Nat Rev 
Neurosci 6:955-965.

Itofusa R, Kamiguchi H (2011) Polarizing membrane dynamics and adhesion for growth cone navigation. Mol Cell Neurosci 48:332-338.

Iwasaki S, Takahashi T (1998) Developmental changes in calcium channel types mediating synaptic transmission in rat auditory brainstem. J Physiol 509 ( Pt 2:419-423.

Jackson D, Holcomb P, Ellisman M, Spirou G (2020) Two types of somatic spines provide sites for intercellular signaling during calyx of Held growth and maturation. Synapse in press.

Jedlicka P, Vlachos A, Schwarzacher SW, Deller T (2008) A role for the spine apparatus in LTP and spatial learning. Behav Brain Res 192:12-19.

Jedrzejczak-Silicka M (2018) History of Cell Culture. In: New Insights into Cell Culture Technology (Gowder SJT, ed), pp 41. InTech.

Joshi I, Shokralla S, Titis P, Wang L-Y (2004) The role of AMPA receptor gating in the development of high-fidelity neurotransmission at the calyx of Held synapse. J Neurosci 24:183-196.

Joshi I, Wang L-Y (2002) Developmental profiles of glutamate receptors and synaptic transmission at a single synapse in the mouse auditory brainstem. J Physiol 540:861-873.

Kakizawa S, Yamasaki M, Watanabe M, Kano M (2000) Critical period for activity-dependent synapse elimination in developing cerebellum. J Neurosci 20:4954-4961.

Kamiya K, Takahashi K, Kitamura K, Momoi T, Yoshikawa Y (2001) Mitosis and apoptosis in postnatal auditory system of the $\mathrm{C} 3 \mathrm{H} / \mathrm{He}$ strain. Brain Res 901:296-302.

Kandler K, Clause A, Noh J (2009) Tonotopic reorganization of developing auditory brainstem circuits. Nat Neurosci 12:711-717.

Kandler K, Friauf E (1993) Pre- and postnatal development of efferent connections of the cochlear nucleus in the rat. J Comp Neurol 328:161-184.

Kang HJ et al. (2013) Spatiotemporal transcriptome of the human brain. Nature 478:483-489.

Kasthuri N et al. (2015) Saturated reconstruction of a volume of neocortex. Cell 162:648-661.

Kasthuri N, Lichtman JW (2003) The role of neuronal identity in synaptic competition. Nature 424:426-430.

Kaufman MH (1992) The Atlas of Mouse Development. San Diego.

Keleman K, Dickson BJ (2001) Short- and long-range repulsion by the Drosophila Unc5 Netrin receptor. Neuron 32:605-617.

Keller-Peck CR, Walsh MK, Gan W, Feng G, Sanes JR, Lichtman JW, Louis S (2001) Asynchronous synapse elimination in neonatal motor units: Studies using GFP transgenic mice. Neuron 31:381-394.

Keller PJ, Stelzer EH (2008) Quantitative in vivo imaging of entire embryos with Digital Scanned Laser Light Sheet Fluorescence Microscopy. Curr Opin Neurobiol 18:624-632.

Kidd T, Bland KS, Goodman CS (1999) Slit is the midline repellent for the Robo receptor in Drosophila. Cell 96:785-794.

Kim G, Kandler K (2003) Elimination and strengthening of glycinergic/GABAergic connections during tonotopic map formation. Nat Neurosci 6:282-290.

Kim JC, Cook MN, Carey MR, Shen C, Regehr WG, Dymecki SM (2009) Linking geneticallydefined neurons to behavior through a broadly applicable silencing allele. Neuron 63:1-17.

Kim M-H, Korogod N, Schneggenburger R, Ho W-K, Lee S-H (2005) Interplay between $\mathrm{Na}+/ \mathrm{Ca} 2+$ exchangers and mitochondria in $\mathrm{Ca} 2+$ clearance at the calyx of Held. J Neurosci 25:6057-6065.

Kitzes LM, Kageyama GH, Semple MN, Kil J (1995) Development of ectopic projections from 
the ventral cochlear nucleus to the superior olivary complex induced by neonatal ablation of the contralateral cochlea. J Comp Neurol 353:341-363.

Klein R (2004) Eph/ephrin signaling in morphogenesis, neural development and plasticity. Curr Opin Cell Biol 16:580-589.

Kochubey O, Han Y, Schneggenburger R (2009) Developmental regulation of the intracellular $\mathrm{Ca} 2+$ sensitivity of vesicle fusion and $\mathrm{Ca} 2+-$ secretion coupling at the rat calyx of Held. J Physiol 587:3009-3023.

Koh CG (2007) Rho GTPases and their regulators in neuronal functions and development. NeuroSignals 15:228-237.

Koike-Tani M, Saitoh N, Takahashi T (2005) Mechanisms underlying developmental speeding in AMPA-EPSC decay time at the calyx of Held. J Neurosci 25:199-207.

Kolodkin AL, Tessier-Lavigne M (2010) Mechanisms and molecules of neuronal wiring: A Primer. Cold Spring Harb Perspect Biol.

Kolson DR, Wan J, Wu J, Dehoff M, Brandebura AN, Qian J, Mathers PH, Spirou GA (2016) Temporal patterns of gene expression during calyx of Held development. Dev Neurobiol 76:166-189.

Kornberg TB, Roy S (2014a) Communicating by touch - neurons are not alone. Trends Cell Biol 24:370-376.

Kornberg TB, Roy S (2014b) Cytonemes as specialized signaling filopodia. Development 141:729-736.

Koundakjian EJ, Appler JL, Goodrich L V. (2007) Auditory neurons make stereotyped wiring decisions before maturation of their targets. J Neurosci 27:14078-14088.

Kronander E, Michalski N, Lebrand C, Hornung J, Schneggenburger R (2017) An organotypic slice culture to study the formation of calyx of Held synapses in vitro. PLoS One 12:1-19.

Krylova O, Herreros J, Cleverley KE, Ehler E, Henriquez JP, Hughes SM, Salinas PC (2002) WNT-3, expressed by motoneurons, regulates terminal arborization of neurotrophin-3responsive spinal sensory neurons. Neuron 35:1043-1056.

Kuwabara N, DiCaprio RA, Zook JM (1991) Afferents to the medial nucleus of the trapezoid body and their collateral projections. J Comp Neurol 314:684-706.

Lallemend F, Sterzenbach U, Hadjab-Lallemend S, Aquino JB, Castelo-Branco G, Sinha I, Villaescusa JC, Levanon D, Wang Y, Franck MCM, Kharchenko O, Adameyko I, Linnarsson S, Groner Y, Turner E, Ernfors P (2012) Positional differences of axon growth rates between sensory neurons encoded by Runx3. EMBO J 31:3718-3729.

Langhorst MF, Schaffer J, Goetze B (2009) Structure brings clarity: Structured illumination microscopy in cell biology. Biotechnol J 4:858-865.

Lecanuet J-P, Schaal B (2002) Sensory performances in the human foetus: A brief summary of research. Intellectica 1:29-56.

Lee CL, Peng BH (2008) The function of mitochonadria in presynatpic development at the neuromuscular junction. Mol Biol Cell 19:150-158.

Lilley BN, Krishnaswamy A, Wang Z, Kishi M, Frank E, Sanes JR (2014) SAD kinases control the maturation of nerve terminals in the mammalian peripheral and central nervous systems. Proc Natl Acad Sci U S A 111:1138-1143.

Lohof AM, Delhaye-Bouchaud N, Mariani J (1996) Synapse elimination in the central nervous system: functional significance and cellular mechanisms. Rev Neurosci 7:85-101.

Longo-Guess C, LH G, Bergstrom D, Johnson K (2007) A missense mutation in the conserved C2B domain of otoferlin causes deafness in a new mouse model of DFNB9. Hear Res 
234:21-28.

López-Muñoz F, Boya J, Alamo C (2006) Neuron theory, the cornerstone of neuroscience, on the centenary of the Nobel Prize award to Santiago Ramón y Cajal. Brain Res Bull 70:391-405.

Lorenzetto E, Caselli L, Feng G, Yuan W, Nerbonne JM, Sanes JR, Buffelli M (2009) Genetic perturbation of postsynaptic activity regulates synapse elimination in developing cerebellum. Proc Natl Acad Sci U S A 106:16475-16480.

Lowery LA, Van Vactor D (2009) The trip of the tip: understanding the growth cone machinery. Nat Rev Mol Cell Biol 10:332-343.

Lu J, Lichtman JW (2007) Imaging the neuromuscular junction over the past centuries. Sheng Li Xue Bao 59:683-696.

Lujan B, Dagostin A, von Gersdorff H (2019) Presynaptic diversity revealed by Ca 2+ permeable AMPA receptors at the Calyx of Held synapse . J Neurosci:2565-18.

Luo L (2002) Actin cytoskeleton regulation in neuronal morphogenesis and structural plasticity. Annu Rev Cell Dev Biol 18:601-635.

Luo L, Hensch TK, Ackerman L, Barbel S, Jan LY, Jan YN (1996) Differential effects of the Rac GTPase on Purkinje cell axons and dendritic trunks and spines. Nature 379:837-840.

Mann F, Chauvet S, Rougon G (2007) Semaphorins in development and adult brain: Implication for neurological diseases. Prog Neurobiol 82:57-79.

Maricich SM, Xia A, Mathes EL, Wang VY, Oghalai JS, Zoghbi HY (2009) Atoh1-lineal neurons are required for hearing and for the survival of neurons in the spiral ganglion and brainstem accessory auditory nuclei. J Neurosci 29:11123-11133.

Marrs GS, Morgan WJ, Howell DM, Spirou G a., Mathers PH (2013) Embryonic origins of the mouse superior olivary complex. Dev Neurobiol 73:384-398.

Marrs GS, Spirou GA (2012) Embryonic assembly of auditory circuits: spiral ganglion and brainstem. J Physiol 590:2391-2408.

Massobrio P, Giachello CN, Ghirardi M, Martinoia S (2013) Selective modulation of chemical and electrical synapses of Helix neuronal networks during in vitro development. BMC Neurosci 14:22.

McCann CM, Lichtman JW (2008) In vivo imaging of presynaptic terminals and postsynaptic sites in the mouse submandibular ganglion. Dev Neurobiol 68:760-770.

McCann CM, Nguyen QT, Santo Neto H, Lichtman JW (2007) Rapid synapse elimination after postsynaptic protein synthesis inhibition in vivo. J Neurosci 27:6064-6067.

McKerracher L, Chamoux M, Arregui CO (1996) Role of laminin and integrin interactions in growth cone guidance. Mol Neurobiol 12:95-116.

McLaughlin T, Hindges R, O’Leary DDM (2003a) Regulation of axial patterning of the retina and its topographic mapping in the brain. Curr Opin Neurobiol 13:57-69.

McLaughlin T, Torborg CL, Feller MB, O’Leary DDM (2003b) Retinotopic map refinement requires spontaneous retinal waves during a brief critical period of development. Neuron 40:1147-1160.

Meister M, Wong ROL, Baylor DA, Shatz CJ (1991) Synchronous bursts of action potentials in ganglion cells of the developing mammalian retina. Science (80- ) 252:939-943.

Meyer AC, Neher E, Schneggenburger R (2001) Estimation of quantal size and number of functional active zones at the calyx of held synapse by nonstationary EPSC variance analysis. J Neurosci 21:7889-7900.

Michalski N, Babai N, Renier N, Perkel DJ, Chédotal A, Schneggenburger R (2013) Robo3driven axon midline crossing conditions functional maturation of a large commissural 
synapse. Neuron 2:1-14.

Mikuni T, Uesaka N, Okuno H, Hirai H, Deisseroth K, Bito H, Kano M (2013) Arc/Arg3.1 is a postsynaptic mediator of activity-dependent synapse elimination in the developing cerebellum. Neuron 78:1024-1035.

Milinkeviciute G, Henningfield CM, Muniak MA, Chokr SM, Green KN, Cramer KS (2019) Microglia regulate pruning of specialized synapses in the auditory brainstem. Front Neural Circuits 13:1-19.

Millard SS, Flanagan JJ, Pappu KS, Wu W, Zipursky SL (2007) Dscam2 mediates axonal tiling in the Drosophila visual system. Nature 447:720-724.

Miller JA et al. (2014) Transcriptional landscape of the prenatal human brain. Nature 508:199206.

Miyazono K, Kamiya Y, Morikawa M (2010) Bone morphogenetic protein receptors and signal transduction. J Biochem 147:35-51.

Montesinos MS, Chen Z, Young SM (2011) pUNISHER: a high-level expression cassette for use with recombinant viral vectors for rapid and long term in vivo neuronal expression in the CNS. J Neurophysiol 106:3230-3244.

Mooney R, Penn AA, Gallego R, Shatz CJ (1996) Thalamic relay of spontaneous retinal activity prior to vision. Neuron 17:979-990.

Morest DK (1968a) The growth of synaptic endings in the mammalian brain: a study of the calyces of the trapezoid body. J Anat Hist Dev 127:201-220.

Morest DK (1968b) The collateral system of the medial nucleus of the trapezoid body of the cat, its neuronal architechture and relation to the olivo-cochlear bundle. Brain Res 9:288-311.

Mortimer D, Fothergill T, Pujic Z, Richards LJ, Goodhill GJ (2008) Growth cone chemotaxis. Trends Neurosci 31:90-98.

Muir-Robinson G, Hwang BJ, Feller MB (2002) Retinogeniculate axons undergo eye-specific segregation in the absence of eye-specific layers. J Neurosci 22:5259-5264.

Müller J, Reyes-Haro D, Pivneva T, Nolte C, Schaette R, Lübke J, Kettenmann H (2009) The principal neurons of the medial nucleus of the trapezoid body and NG2+ glial cells receive coordinated excitatory synaptic input. J Gen Physiol 134:115-127.

Müller MK, Jovanovic S, Keine C, Radulovic T, Rübsamen R, Milenkovic I (2019) Functional development of principal neurons in the anteroventral cochlear nucleus extends beyond hearing onset. Front Cell Neurosci 13:1-21.

Muniak MA, Rivas A, Montey KL, May BJ, Francis HW, Ryugo DK (2013) 3D model of frequency representation in the cochlear nucleus of the CBA/J mouse. J Comp Neurol $521: 1510-1532$.

Mural RJ et al. (2002) A Comparison of whole-genome shotgun-derived mouse chromosome 16 and the human Genome. Science (80- ) 296:1661-1671.

Murray SA, Morgan JL, Kane C, Sharma Y, Heffner CS, Lake J, Donahue LR (2010) Mouse gestation length is genetically determined. PLoS One 5.

Nakagawa S, Brennan C, Johnson KG, Shewan D, Harris WA, Holt CE (2000) Ephrin-B regulates the ipsilateral routing of retinal axons at the optic chiasm. Neuron 25:599-610.

Nakamura PA, Cramer KS (2011) Formation and maturation of the calyx of Held. Hear Res 276:70-78.

Nakamura PA, Cramer KS (2013) EphB2 signaling regulates lesion-induced axon sprouting but not critical period length in the postnatal auditory brainstem. Neural Dev 8:1-11.

Nakayama AY, Harms MB, Luo L (2000) Small GTPases Rac and Rho in the maintenance of 
dendritic spines and branches in hippocampal pyramidal neurons. J Neurosci 20:5329-5338.

Niessen CM, Gottardi CJ (2008) Molecular components of the adherens junction. Biochim Biophys Acta - Biomembr 1778:562-571.

Nimchinsky EA, Sabatini BL, Svoboda K (2002) Structure and function of dendritic spines. Annu Rev Physiol 64:313-353.

Noctor SC, Martinez-Cerdeño V, Ivic L, Kriegstein AR (2004) Cortical neurons arise in symmetric and asymmetric division zones and migrate through specific phases. Nat Neurosci 7:136-144.

Noguchi J, Matsuzaki M, Ellis-Davies GCR, Kasai H (2005) Spine neck geometry determines NMDA receptor-dependent CA2+ signaling in dendrites. Neuron 46:609-622.

Noh J, Seal RP, Garver JA, Edwards RH, Kandler K (2010) Glutamate co-release at GABA/glycenergic synapses is crucial for the refinement of an inhibitory map. Nat Neurosci 13:232-238.

O'Brien J, Unwin N (2006) Organization of spines on the dendrites of Purkinje cells. Proc Natl Acad Sci U S A 103:1575-1580.

O'Donnell M, Chance RK, Bashaw GJ (2009) Axon growth and guidance: receptor regulation and signal transduction. Annu Rev Neurosci 32:383-412.

O’Rahilly R, Müller F (2007) The development of the neural crest in the human. J Anat 211:335-351.

O'Rourke NA, Cline HT, Fraser SE (1994) Rapid remodeling of retinal arbors in the tectum with and without blockade of synaptic transmission. Neuron 12:921-934.

Obrien RAD, Ostberg JC, Vrbova G (1978) Observations on the elimination of polyneuronal innervation in the developing mammalian skelatal muscle. J Physiol 282:571-582.

Okuno H, Akashi K, Ishii Y, Yagishita-Kyo N, Suzuki K, Nonaka M, Kawashima T, Fujii H, Takemoto-Kimura S, Abe M, Natsume R, Chowdhury S, Sakimura K, Worley PF, Bito H (2012) Inverse synaptic tagging of inactive synapses via dynamic interaction of Arc/Arg3.1 with CaMKII $\beta$. Cell 149:886-898.

Ooyen A Van (2005) Competition in neurite outgrowth and the development of nerve connections. Prog Brain Res 147:81-99.

Oster SF, Bodeker MO, He F, Sretavan DW (2003) Invariant Sema5A inhibition serves an ensheathing function during optic nerve development. Development 130:775-784.

Özel MN, Langen M, Hassan BA, Hiesinger PR (2015) Filopodial dynamics and growth cone stabilization in Drosophila visual circuit development. Elife 4:1-21.

Parker I, Evans KT, Ellefsen K, Lawson DA, Smith IF (2017) Lattice light sheet imaging of membrane nanotubes between human breast cancer cells in culture and in brain metastases. Sci Rep 7:1-7.

Pearson BJ, Doe CQ (2004) Specification of temporal identity in the developing nervous system. Annu Rev Cell Dev Biol 20:619-647.

Penn AA, Riquelme PA, Feller MB, Shatz CJ (1998) Competition in retinogeniculate patterning driven by spontaneous activity. Science (80- ) 279:2108-2112.

Perälä N, Sariola H, Immonen T (2012) More than nervous: the emerging roles of plexins. Differentiation 83:77-91.

Petralia RS, Wang Y-X, Mattson MP, Yao PJ (2015) Structure, distribution, and fuction of neuronal/synaptic spinules and related invaginating projections. NeuroMolecular Med 17:211-240.

Petralia RS, Wang YX, Mattson MP, Yao PJ (2018) Invaginating structures in mammalian 
synapses. Front Synaptic Neurosci 10:1-8.

Pfeffer W (1884) Locomotorische Richtungsbewegungen durch chemische Reize.

Untersuchungen ails dem Bot Inst Tiibingen 1:363-482.

Pfeffer W (1888) Uber Chemotaktische Bewegungen von Bacterien, Flagellaten, und Volvocineen. Untersuchungen ails dem Bot Inst Tiibingen 2:582-661.

Phan KD, Hazen VM, Frendo M, Jia Z, Butler SJ (2010) The bone morphogenetic protein roof plate chemorepellent regulates the rate of commissural axonal growth. J Neurosci 30:15430-15440.

Pierce ET (1967) Histogenesis of the dorsal and ventral cochlear nuclei in the mouse. An autoradiographic study. J Comp Neurol 131:27-53.

Planchon TA, Liang G, Milkie DE, Davidson MW, Galbraith JA, Galbraith CG, Betzig E (2011) Rapid three-dimensional isotropic imaging of living cells using Bessel beam plane illumination. Nat Methods 8:417-423.

Pollard TD, Cooper JA (2009) Actin, a central player in cell shape and movement. Science (80- ) 326:1208-1212.

Poon VY, Choi S, Park M (2013) Growth factors in synaptic function. Front Synaptic Neurosci 5:1-6.

Popov VI, Kleschevnikov AM, Klimenko OA, Stewart MG, Belichenko P V. (2011) Threedimensional synaptic ultrastructure in the dentate gyrus and hippocampal area CA3 in the Ts65Dn mouse model of down syndrome. J Comp Neurol 519:1338-1354.

Portera-Cailliau C, Weimer RM, De Paola V, Caroni P, Svoboda K (2005) Diverse modes of axon elaboration in the developing neocortex. PLoS Biol 3.

Rajnicek AM, Britland S, McCaig CD (1997) Contact guidance of CNS neurites on grooved quartz: influence of groove dimensions, neuronal age and cell type. J Cell Sci 110:29052913.

Rakic P, Sidman RL (1973) Organization of cerebellar cortex secondary to deficit of granule cells in weaver mutant mice. J Comp Neurol 152:133-161.

Ramírez-Weber FA, Kornberg TB (1999) Cytonemes: Cellular processes that project to the principal signaling center in Drosophila imaginal discs. Cell 97:599-607.

Ramón y Cajal S (1890) Notas anatómicas. I. Sobre la aparición de las expansiones celulares en la médula embrionaria. Gac Sanit Barcelona 12:413-419.

Ramón y Cajal S (1892) The retina of vertebrates. Cell 9:121-255.

Ramón y Cajal S (1911) Histologie du systéme nerveux de l' homme et des vertébrés. Paris: Maloine.

Raper J, Mason C (2010) Cellular strategies of axonal pathfinding. Cold Spring Harb Perspect Biol 2:a001933.

Reyes-Haro D, Müller J, Boresch M, Pivneva T, Benedetti B, Scheller A, Nolte C, Kettenmann $\mathrm{H}$ (2010) Neuron-astrocyte interactions in the medial nucleus of the trapezoid body. J Gen Physiol 135:583-594.

Rietzel HJ, Friauf E (1998) Neuron types in the rat lateral superior olive and developmental changes in the complexity of their dendritic arbors. J Comp Neurol 390:20-40.

Rink J, Ghigo E, Kalaidzidis Y, Zerial M (2005) Rab conversion as a mechanism of progression from early to late endosomes. Cell 122:735-749.

Rios O, Villalobos J (2004) Postnatal development of the afferent projections from the dorsomedial thalamic nucleus to the frontal cortex in mice. Dev Brain Res 150:47-50.

Rodríguez-Contreras A, de Lange RPJ, Lucassen PJ, Borst JGG (2006) Branching of calyceal 
afferents during postnatal development in the rat auditory brainstem. J Comp Neurol 496:214-228.

Rodríguez-Contreras A, van Hoeve JSS, Habets RLP, Locher H, Borst JGG (2008) Dynamic development of the calyx of Held synapse. Proc Natl Acad Sci U S A 105:5603-5608.

Rodríguez-Hernández CO, Torres-García SE, Olvera-Sandoval C, Ramírez-Castillo FY, Muro AL, Avelar-Gonzalez FJ, Guerrero-Barrera AL (2014) Cell Culture: History, development and prospects. Int J Curr Res Acad Rev 2:188-200.

Rojas-Ríos P, Guerrero I, González-Reyes A (2012) Cytoneme-mediated delivery of Hedgehog regulates the expression of bone morphogenetic proteins to maintain germline stem cells in Drosophila. PLoS Biol 10.

Romand R, Ehret G (1990) Development of tonotopy in the inferior colliculus. I. Electrophysiological mapping in house mice. Dev Brain Res 54:221-234.

Roux I, Safieddine S, Nouvian R, Grati M, Simmler MC, Bahloul A, Perfettini I, Le Gall M, Rostaing P, Hamard G, Triller A, Avan P, Moser T, Petit C (2006) Otoferlin, defective in a human deafness form, is essential for exocytosis at the auditory ribbon synapse. Cell 127:277-289.

Rowland KC, Irby NK, Spirou GA (2000) Specialized synapse-associated structures within the calyx of Held. J Neurosci 20:9135-9144.

Roy S, Hsiung F, Kornberg TB (2012) Specificity of Drosophila cytonemes for distinct signaling pathways. Bone 23:1-7.

Roy S, Zhang B, Lee VMY, Trojanowski JQ (2005) Axonal transport defects: A common theme in neurodegenerative diseases. Acta Neuropathol 109:5-13.

Rubio ME, Nagy JI (2015) Connexin36 expression in major centers of the auditory system in the CNS of mouse and rat: Evidence for neurons forming purely electrical synapses and morphologically mixed synapses. Neuroscience 303:604-629.

Rudolph S, Tsai MC, von Gersdorff H, Wadiche JI (2015) The ubiquitous nature of multivesicular release. Trends Neurosci 38:428-438.

Ruel J, Emery S, Nouvian R, Bersot T, Amilhon B, Van Rybroek JM, Rebillard G, Lenoir M, Eybalin M, Delprat B, Sivakumaran T a., Giros B, El Mestikawy S, Moser T, Smith RJH, Lesperance MM, Puel JL (2008) Impairment of SLC17A8 encoding vesicular glutamate transporter-3, VGLUT3, underlies nonsyndromic deafness DFNA25 and inner hair cell dysfunction in null mice. Am J Hum Genet 83:278-292.

Russell FA, Moore DR (1995) Afferent reorganisation within the superior olivary complex of the gerbil: Development and induction by neonatal, unilateral cochlear removal. J Comp Neurol 352:607-625.

Rusu SI, Borst JGG (2011) Developmental changes in intrinsic excitability of principal neurons in the rat medial nucleus of the trapezoid body. Dev Neurobiol 71:284-295.

Ruthazer ES, Bachleda AR, Olavarria JF (2010) Role of interstitial branching in the dvelopment of the visual corticospinal connections: a time-lapse and fixed-tissue analysis. J Comp Neurol 518.

Sabry JH, O'Connor TP, Evans L, Toroian-Raymond A, Kirschner M, Bentley D (1991) Microtubule behavior during guidance of pioneer neuron growth cones in situ. J Cell Biol 115:381-395.

Saliu A, Adise S, Xian S, Kudelska K, Rodríguez-Contreras A (2014) Natural and lesioninduced decrease in cell proliferation in the medial nucleus of the trapezoid body during hearing development. J Comp Neurol 522:971-985. 
Sanculi D, Pannoni KE, Bushong EA, Crump M, Sung M, Popat V, Zaher C, Hicks E, Song A, Mofakham N, Li P, Antzoulatos EG, Fioravante D, Ellisman MH, DeBello WM (2020) Toric spines at a site of learning. eNeuro 7:1-23.

Sanes DH, Takács C (1993) Activity-dependent refinement of inhibitory connections. Eur J Neurosci 5:570-574.

Sanes JR, Lichtman JW (1999) Development of the vertebrate neuromuscular junction. Annu Rev Neurosci 22:389-442.

Sanes JR, Lichtman JW (2001) Induction, assembly, maturation and maintenance of a postsynaptic apparatus. Nat Rev Neurosci 2:791-805.

Sanes JR, Yamagata M (2009) Many paths to synaptic specificity. Annu Rev Cell Dev Biol 25:161-195.

Sätzler K, Söhl LF, Bollmann JH, Borst JGG, Frotscher M, Sakmann B, Lübke JHR (2002) Three-dimensional reconstruction of a calyx of Held and its postsynaptic principal neuron in the medial nucleus of the trapezoid body. J Neurosci 22:10567-10579.

Schiavo G, Matteoli M, Montecucco C (2000) Neurotoxins affecting neuroexocytosis. Physiol Rev 80:717-766.

Schlosshauer B, Müller E, Schröder B, Planck H, Müller HW (2003) Rat Schwann cells in bioresorbable nerve guides to promote and accelerate axonal regeneration. Brain Res 963:321-326.

Schoch S, Deák F, Königstorfer A, Mozhayeva M, Sara Y, Südhof TC, Kavalali ET (2001) SNARE function analyzed in synaptobrevin/VAMP knockout mice. Science (80- ) 294:1117-1122.

Schuldiner O, Yaron A (2014) Mechanisms of developmental neurite pruning. Cell Mol Life Sci 72:101-119.

Schwarting GA, Henion TR (2011) Regulation and function of axon guidance and adhesion molecules during olfactory map formation. J Cell Biochem 112:2663-2671.

Seal R, Akil O, Yi E, Weber C, Grant L, Yoo J, Clause A, Kandler K, Noebels J, Glowatzki E, Lustig L, Edwards R (2008) Sensorineural deafness and seizures in mice lacking vesicular glutamate transporter 3. Neuron 57:263-275.

Seeger M, Tear G, Ferres-Marco D, Goodman CS (1993) Mutations affecting growth cone guidance in drosophila: Genes necessary for guidance toward or away from the midline. Neuron 10:409-426.

Serafini T, Kennedy TE, Gaiko MJ, Mirzayan C, Jessell TM, Tessier-Lavigne M (1994) The netrins define a family of axon outgrowth-promoting proteins homologous to $\mathrm{C}$. elegans UNC-6. Cell 78:409-424.

Serafini TA, Colamarino SA, Leonardo DE, Wang H, Beddington R, Skarnes WC, TessierLavigne M (1996) Netrin-1 is required for commissural axon guidance in the developing vertebrate nervous system. Cell 87:1001-1014.

Shatz CJ, Stryker MP (1988) Prenatal tetrodotoxin infusion blocks segregation of retinogeniculate afferents. Science (80- ) 242:87-89.

Shoop RD, Esquenazi E, Yamada N, Ellisman MH, Berg DK (2002) Ultrastructure of a somatic spine mat for nicotinic signaling in neurons. J Neurosci 22:748-756.

Shoop RD, Martone ME, Yamada N, Ellisman MH, Berg DK (1999) Neuronal acetylcholine receptors with $\alpha 7$ subunits are concentrated on somatic spines for synaptic signaling in embryonic chick ciliary ganglia. J Neurosci 19:692-704.

Sierksma MC, Tedja MS, Borst JGG (2017) In vivo matching of postsynaptic excitability with 
spontaneous synaptic inputs during formation of the rat calyx of Held synapse. J Physiol 595:207-231.

Smith PH, Joris PX, Carney LH, Yin TC (1991) Projections of physiologically characterized globular bushy cell axons from the cochlear nucleus of the cat. J Comp Neurol 304:387407.

Smith PH, Joris PX, Yin TCT (1993) Projections of physiologically characterized spherical bushy cell axons from the cochlear nucleus of the cat: Evidence for delay lines to the medial superior olive. J Comp Neurol 331:245-260.

Snyder JM, Hagan CE, Bolan B, Keene CD (2018) 20 - Nervous System. In: Comparative Anatomy (2nd Edition), pp 4347-4352. American Chemical Society.

Song H, Poo M (1999) Signal transduction underlying growth cone guidance by diffusible factors. Curr Opin Neurobiol 9:355-363.

Sonntag M, Englitz B, Kopp-Scheinpflug C, Rübsamen R (2009) Early postnatal development of spontaneous and acoustically evoked discharge activity of principal cells of the medial nucleus of the trapezoid body: An in vivo study in mice. J Neurosci 29:9510-9520.

Spacek J, Harris KM (2004) Trans-endocytosis via spinules in adult rat hippocampus. J Neurosci 24:4233-4241.

Sperry RW (1963) Chemoaffinity in the orderly growth of nerve fiber patterns and connections. Proc Natl Acad Sci 50:703-710.

Spirou GA, Brownell WE, Zidanic M (1990) Recordings from cat trapezoid body and HRP labeling of globular bushy cell axons. J Neurophysiol 63:1169-1190.

Spirou GA, Rager J, Manis PB (2005) Convergence of auditory-nerve fiber projections onto globular bushy cells. Neuroscience 136:843-863.

Stanganello E, Hagemann AIH, Mattes B, Sinner C, Meyen D, Weber S, Schug A, Raz E, Scholpp S (2015) Filopodia-based Wnt transport during vertebrate tissue patterning. Nat Commun 6:5846.

Stellwagen D, Shatz CJ (2002) An instructive role for retinal waves in the development of retinogeniculate connectivity. Neuron 33:357-367.

Stockard CR (1921) Developmental rate and structural expression: an experimental study of twin, "double monsters" and single deformities, and the interaction among embryonic organs during their origin and development. Am Jounal Anat 28:115-227.

Sugihara I (2005) Microzonal projection and climbing fiber remodeling in single olivocerebellar axons of newborn rats at postnatal days 4-7. J Comp Neurol 487:93-106.

Sun KLW, Correia JP, Kennedy TE (2011) Netrins: versatile extracellular cues with diverse functions. Development 138:2153-2169.

Suter DM, Forscher P (2000) Substrate-cytoskeletal coupling as a mechanism for the regulation of growth cone motility and guidance. J Neurobiol 44:97-113.

Szebenyi G, Callaway JL, Dent EW, Kalil K (1998) Interstitial branches develop from active regions of the axon demarcated by the primary growth cone during pausing behaviors. $\mathrm{J}$ Neurosci 18:7930-7940.

Takahashi T (2015) Strength and precision of neurotransmission at mammalian presynaptic terminals. Proc Jpn Acad Ser B Phys Biol Sci 91:305-320.

Tamagnone L, Comoglio PM (2000) Signalling by semaphorin receptors: Cell guidance and beyond. Trends Cell Biol 10:377-383.

Tanaka E, Ho T, Kirschner MW (1995) The role of microtubule dynamics in growth cone motility and axonal growth. J Cell Biol 128:139-155. 
Tao-Cheng JH, Dosemeci A, Gallant PE, Miller S, Galbraith JA, Winters CA, Azzam R, Reese TS (2009) Rapid turnover of spinules at synaptic terminals. Neuroscience 160:42-50.

Tapia JC, Wylie JD, Kasthuri N, Hayworth KJ, Schalek R, Berger DR, Guatimosim C, Seung HS, Lichtman JW (2012) Pervasive synaptic branch removal in the mammalian neuromuscular system at birth. Neuron 74:816-829.

Tardivel M, Bégard S, Buée L, Coens A, Melki R, Dujardin S, Bousset L, Colin M (2016) Tunneling nanotube (TNT)-mediated neuron-to neuron transfer of pathological Tau protein assemblies. Acta Neuropathol Commun 4:1-14.

Taschenberger H, Leão RM, Rowland KC, Spirou GA, von Gersdorff H (2002) Optimizing synaptic architecture and efficiency for high-frequency transmission. Neuron 36:11271143.

Taschenberger H, von Gersdorff H (2000) Fine-tuning an auditory synapse for speed and fidelity: developmental changes in presynaptic waveform, EPSC kinetics, and synaptic plasticity. J Neurosci 20:9162-9173.

Tashiro A, Dunaevsky A, Blazeski R, Mason CA, Yuste R (2003) Bidirectional regulation of hippocampal mossy fiber filopodial motility by kainate receptors: A two-step model of synaptogenesis. Neuron 38:773-784.

Tessier-Lavigne M, Goodman CS (1996) The molecular biology of axon guidance. Science (80) 274:1123-1133.

Thompson W (1983) Synapse elimination in neonatal rat muscle is sensitive to pattern of muscle use. Nature 302:614-616.

Tintignac LA, Brenner H-R, Rüegg MA (2015) Mechanisms regulating neuromuscular junction development and function and causes of muscle wasting. Physiol Rev 95:809-852.

Tojima T, Itofusa R, Kamiguchi H (2014) Steering neuronal growth cones by shifting the imbalance between exocytosis and endocytosis. J Neurosci 34:7165-7178.

Tojima T, Kamiguchi H (2015) Exocytic and endocytic membrane trafficking in axon development. Dev Growth Differ 57:291-304.

Tolbert LP, Morest DK (1982) The neuronal architecture of the anteroventral cochlear nucleus of the cat in the region of the cochlear nerve root: electron microscopy. Neuroscience 7:30533067.

Tollin DJ (2003) The lateral superior olive: A functional role in sound source localization. Neuroscientist 9:127-143.

Tomer R, Lovett-Barron M, Kauvar I, Andalman A, Burns VM, Sankaran S, Grosenick L, Broxton M, Yang S, Deisseroth K (2015) SPED light sheet microscopy: Fast mapping of biological system structure and function. Cell 163:1796-1806.

Torborg CL, Feller MB (2005) Spontaneous patterned retinal activity and the refinement of retinal projections. Prog Neurobiol 76:213-235.

Torre V, Shahapure RB, Di Fabrizio EM, Difato F, Ferrari E, Cojoc D, Righi M, Laishram J (2007) Properties of the force exerted by filopodia and lamellipodia and the involvement of cytoskeletal components. PLoS One 2:e1072.

Toyoshima M, Sakurai K, Shimazaki K, Takeda Y, Shimoda Y, Watanabe K (2009) Deficiency of neural recognition molecule NB-2 affects the development of glutamatergic auditory pathways from the ventral cochlear nucleus to the superior olivary complex in mouse. Dev Biol 336:192-200.

Tritsch NX, Bergles DE (2010) Developmental regulation of spontaneous activity in the Mammalian cochlea. J Neurosci 30:1539-1550. 
Tritsch NX, Rodríguez-Contreras A, Crins TTH, Wang HC (2010) Calcium action potentials in hair cells pattern auditory neuron activity before hearing onset. Nat Neurosci 13:10501052.

Tritsch NX, Yi E, Gale JE, Glowatzki E, Bergles DE (2007) The origin of spontaneous activity in the developing auditory system. Nature 450:50-55.

Trussell LO (1999) Synaptic mechanisms for coding timing in auditory neurons. Annu Rev Physiol 61:477-496.

Turney SG, Lichtman JW (2012) Reversing the outcome of synapse elimination at developing neuromuscular junctions in vivo: evidence for synaptic competition and its mechanism. PLoS Biol 10:e1001352.

Udin SB, Fawcett JW (1988) Formation of topographic maps. Annu Rev Neurosci 11:289-327.

Van Hoeve JSS, Gerard J, Borst G (2010) Delayed appearance of the scaffolding proteins PSD95 and homer-1 at the developing rat calyx of Held synapse. J Comp Neurol 518:45814590.

Varoqueaux F, Sigler A, Rhee J-S, Brose N, Enk C, Reim K, Rosenmund C (2002) Total arrest of spontaneous and evoked synaptic transmission but normal synaptogenesis in the absence of Munc13-mediated vesicle priming. Proc Natl Acad Sci U S A 99:9037-9042.

Vidulescu C, Clejan S, O'Connor KC (2004) Vesicle traffic through intercellular bridges in DU 145 human prostate cancer cells. J Cell Mol Med 8:388-396.

Vilmont V, Cadot B, Ouanounou G, Gomes ER (2016) A system for studying mechanisms of neuromuscular junction development and maintenance. Development 143:2464-2477.

von Gersdorff H, Borst JGG (2002) Short-term plasticity at the calyx of held. Nat Rev Neurosci 3:53-64.

Walmsley B, Alvarez FJ, Fyffe REW (1998) Diversity of structure and function at mammalian central synapses. Trends Neurosci 21:81-88.

Walsh MK, Lichtman JW (2003) In vivo time-lapse imaging of synaptic takeover associated with naturally occurring synapse elimination. Neuron 37:67-73.

Wang L-Y, Neher E, Taschenberger H (2008) Synaptic vesicles in mature calyx of Held synapses sense higher nanodomain calcium concentrations during action potential-evoked glutamate release. J Neurosci 28:14450-14458.

Wang L, Brown A (2002) Rapid movement of microtubules in axons. Curr Biol 12:1496-1501.

Wang LY, Gan L, Forsythe ID, Kaczmarek LK (1998) Contribution of the Kv3.1 potassium channel to high frequency firing in mouse auditory neurones. J Physiol 509:183-194.

Wang Y, Cui J, Sun X, Zhang Y (2011) Tunneling-nanotube development in astrocytes depends on p53 activation. Cell Death Differ 18:732-742.

Watanabe M, Kano M (2011) Climbing fiber synapse elimination in cerebellar Purkinje cells. Eur J Neurosci 34:1697-1710.

Weatherstone JH, Kopp-Scheinpflug C, Pilati N, Wang Y, Forsythe ID, Rubel EW, Tempel BL (2016) Maintenance of neuronal size gradient in MNTB requires sound-evoked activit. J Neurophysiol 117:jn.00528.2016.

Wei C, Thatcher EJ, Olena AF, Cha DJ, Perdigoto AL, Marshall AF, Carter BD, Broadie K, Patton JG (2013) miR-153 regulates SNAP-25, synaptic transmission, and neuronal development. PLoS One 8:e57080.

Weigel S, Osterwalder T, Tobler U, Yao L, Wiesli M, Lehnert T, Pandit A, Bruinink A (2012) Surface microstructures on planar substrates and textile fibers guide neurite outgrowth: A scaffold solution to push limits of critical nerve defect regeneration? PLoS One 7. 
Westrum LE, Blackstad TW (1962) An electron microscopic study of the stratum radiatum of the rat hippocampus (regio superior, CA 1) with particular emphasis on synaptology. J Comp Neurol 119:281-309.

White JG, Southgate E, Thomson JN, Brenner S (1986) The structure of the nervous system of the nematode caenorhabditis elegans. Philos Trans R Soc Lond B Biol Sci 314:1-340.

Whitlon DS, Zhang X, Pecelunas K, Greiner MA (1999) A temporospatial map of adhesive molecules in the organ of Corti of the mouse cochlea. J Neurocytol 28:955-968.

Wiesel TN, Hubel DH (1965) Comparison of the effects of unilateral and bilateral eye closure on cortical unit responses in kittens. J Neurophysiol 28:1029-1040.

Wilkinson DG (2001) Multiple roles of EPH receptors and ephrins in neural development. Nat Rev Neurosci 2:155-164.

Williams ME, de Wit J, Ghosh A (2010) Molecular mechanisms of synaptic specificity in developing neural circuits. Neuron 68:9-18.

Williams SE, Mann F, Erskine L, Sakurai T, Wei S, Rossi DJ, Gale NW, Holt CE, Mason CA, Henkemeyer M (2003) Ephrin-B2 and EphB1 mediate retinal axon divergence at the optic chiasm. Neuron 39:919-935.

Witte S, Stier H, Cline HT (1996) In vivo observations of timecourse and distribution of morphological dynamics in Xenopus retinotectal axon arbors. J Neurobiol 31:219-234.

Wong K, Park HT, Wu JY, Rao Y (2002) Slit proteins: molecular guidance cues for cells ranging from neurons to leukocytes. Curr Opin Genet Dev 12:583-591.

Wong RO, Meister M, Shatz CJ (1993) Transient period of correlated bursting activity during development of the mammalian retina. Neuron 11:923-938.

Woolfrey KM, Srivastava DP (2016) Control of dendritic spine morphological and functional plasticity by small GTPases. Neural Plast 2016.

Wu H, Xiong WC, Mei L (2010) To build a synapse: signaling pathways in neuromuscular junction assembly. Development 137:1017-1033.

Xiao L, Michalski N, Kronander E, Gjoni E, Genoud C, Knott G, Schneggenburger R (2013) BMP signaling specifies the development of a large and fast CNS synapse. Nat Neurosci 16:1-11.

Yamamoto M, Wada N, Kitabatake Y, Watanabe D, Anzai M, Yokoyama M, Teranishi Y, Nakanishi S (2003) Reversible suppression of glutamatergic neurotransmission of cerebellar granule cells in vivo by genetically manipulated expression of tetanus neurotoxin light chain. J Neurosci 23:6759-6767.

Yamauchi T (2005) Neuronal Ca2+/calmodulin-dependent protein kinase II--discovery, progress in a quarter of a century, and perspective: implication for learning and memory. Biol Pharm Bull 28:1342-1354.

Yang Y, Wang L-Y (2006) Amplitude and kinetics of action potential-evoked Ca2+ current and its efficacy in triggering transmitter release at the developing calyx of Held synapse. $\mathrm{J}$ Neurosci 26:5698-5708.

Youssoufian M (2005) Development of a robust central auditory synapse in congenital deafness. J Neurophysiol 94:3168-3180.

Yu W-M, Goodrich L V. (2014) Morphological and physiological development of auditory synapses. Hear Res 311:3-16.

Yuste R (2015) The discovery of dendritic spines by Cajal. Front Neuroanat 9:1-6.

Yuste R, Bonhoeffer T (2004) Genesis of dendritic spines: Insights from ultrastructural and imaging studies. Nat Rev Neurosci 5:24-34. 
Zancan M, Dall'Oglio A, Sarzenski TM, Maher MI, Garcia-Segura LM, Rasia-Filho AA (2015) Glial and axonal perikaryal coverage and somatic spines in the posterodorsal medial amygdala of male and cycling female rats. J Comp Neurol 523:2127-2137.

Zhang B, Xiong WC, Mei L (2009) Get ready to Wnt: Prepatterning in neuromuscular junction formation. Dev Cell 16:325-327.

Zhong J, Zou H (2014) BMP signaling in axon regeneration. Curr Opin Neurobiol:127-134.

Zou Y, Lyuksyutova AI (2007) Morphogens as conserved axon guidance cues. Curr Opin Neurobiol 17:22-28. 
Figures

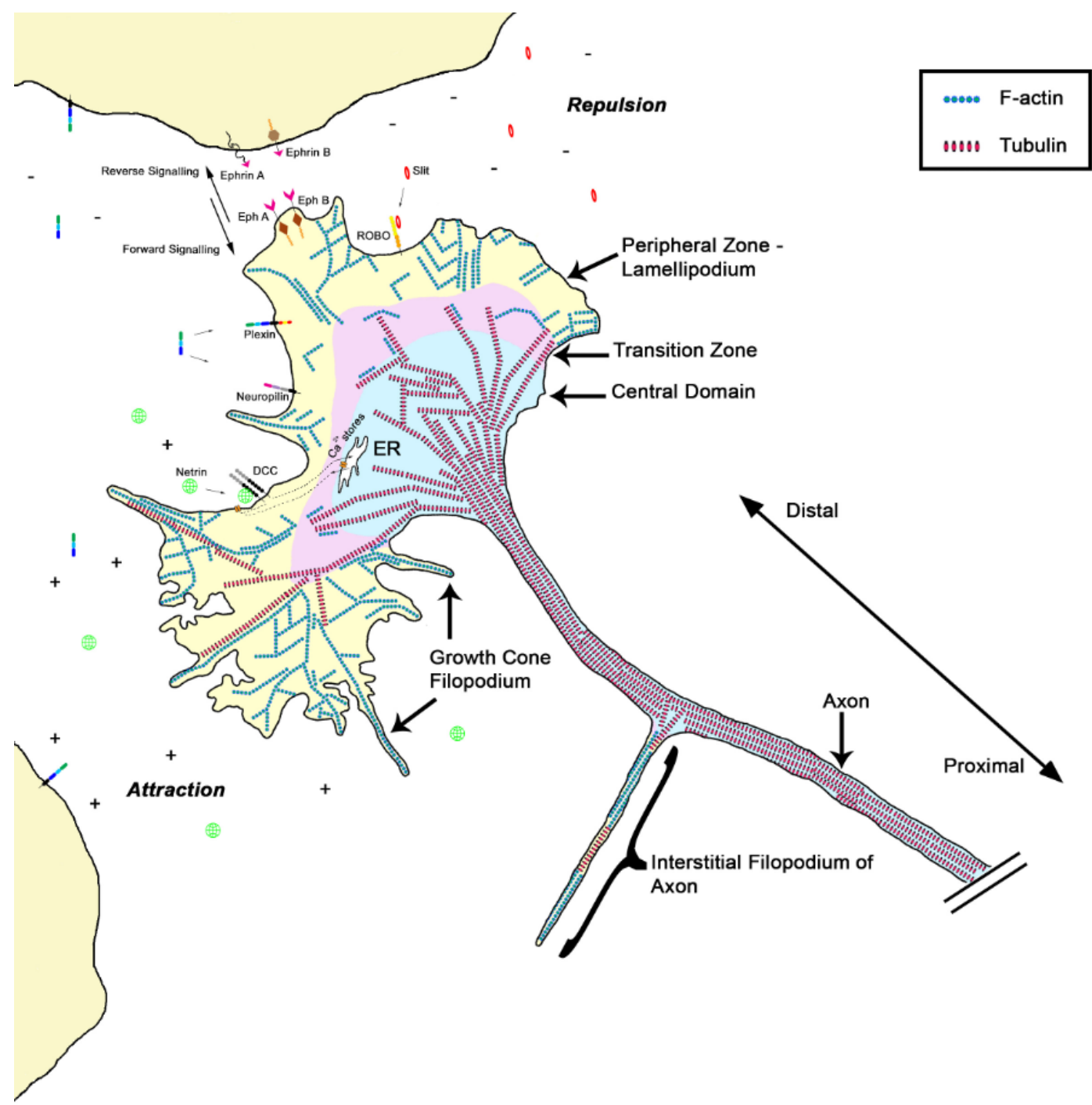

Chapter 1, Figure 1. Growth cone motility is highly regulated to balance asynchronies in endocytosis and exocytosis, as well maintain dynamic stability of cytoskeletal elements. Permissive cues of chemoattraction tilt balance of these forces to steer outgrowth of the growth cone towards the attractant signal 
Figures

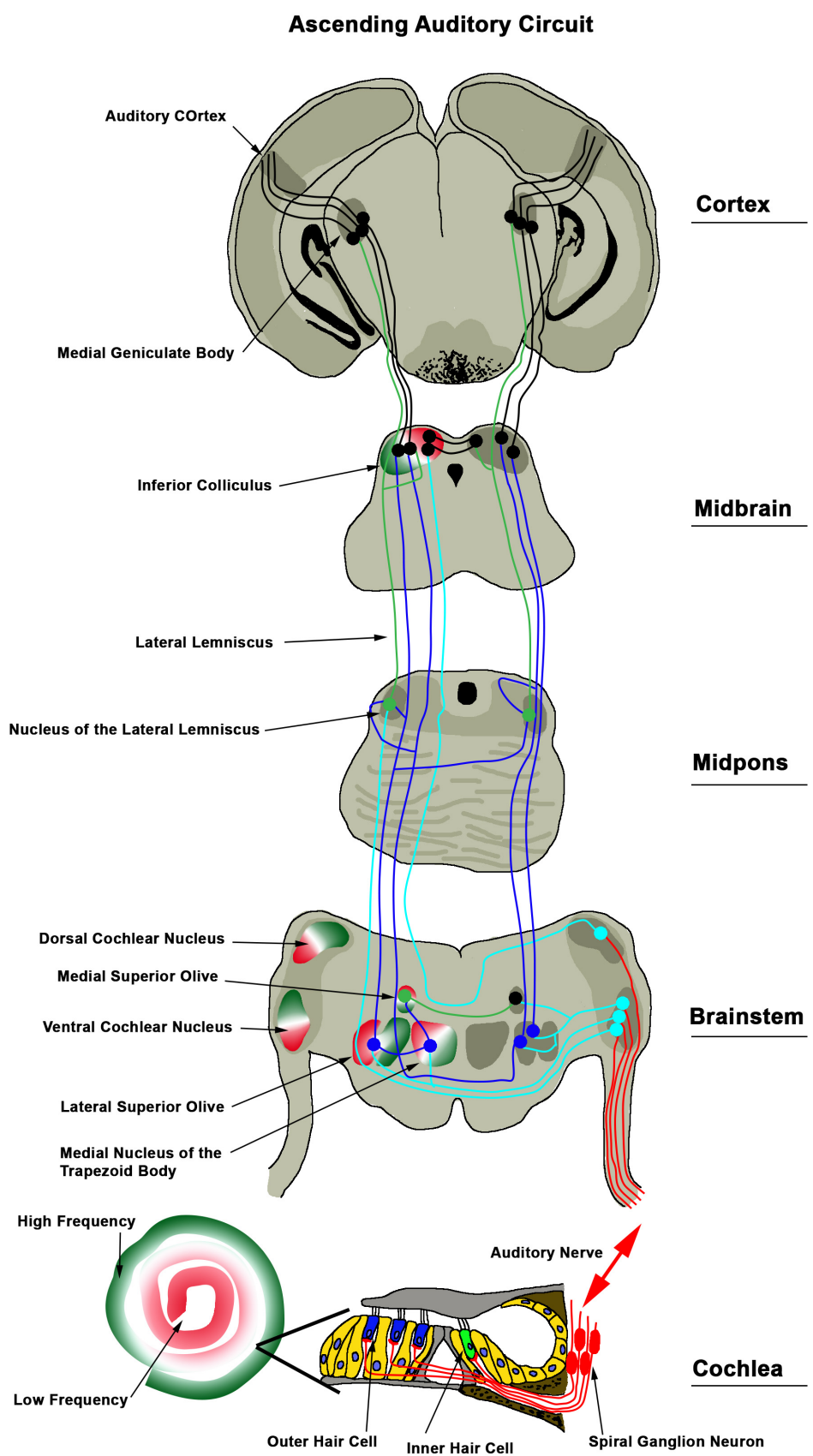

Chapter 1, Figure 2. The organization of the auditory system has evolved to maintain tonotopic information relevant for frequency discrimination 
Figures
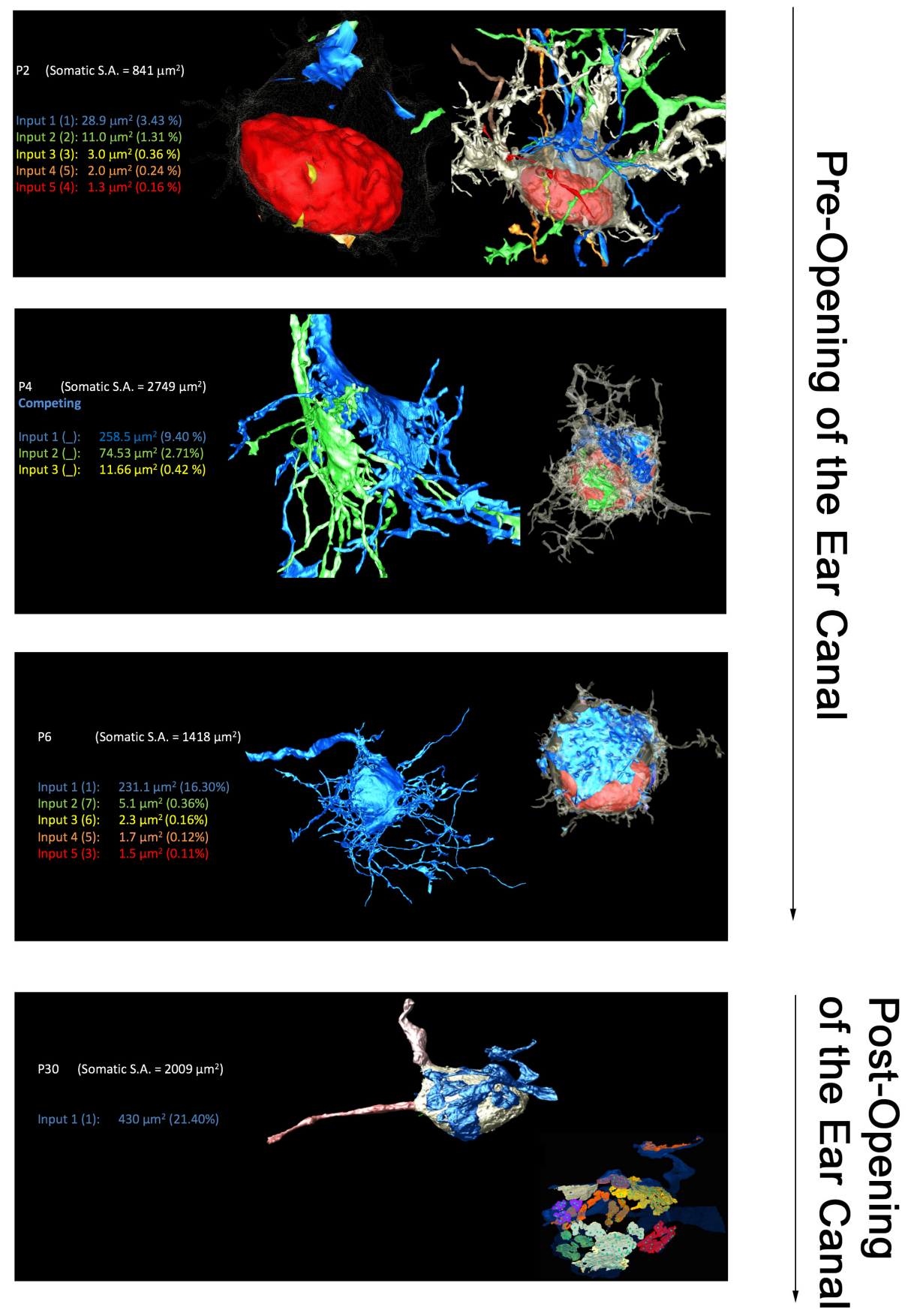

Chapter 1, Figure 3. Ultrastructural reconstructions of developing GBC terminals in the neonatal MNTB (Holcomb et al., 2013) have been useful in investigating the stereotypical formation of a large nerve terminal. During the first postnatal week, 2 or 3 bouton-type terminals present from birth are permitted to grow large. Convergent inputs compete to establish a the largest apposed surface area possible across the somatic surface of its target, the PC of the MNTB. However, following the opening of the ear canal ( P10), the large spoon-like protocalyx will undergo its final structural transformation, achieve the mature fenestrated morphology. 
Figures
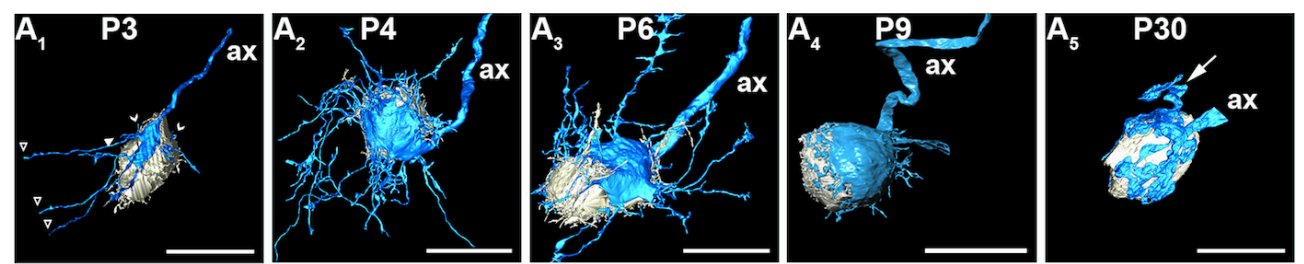

pre opening of ear canal post hearing
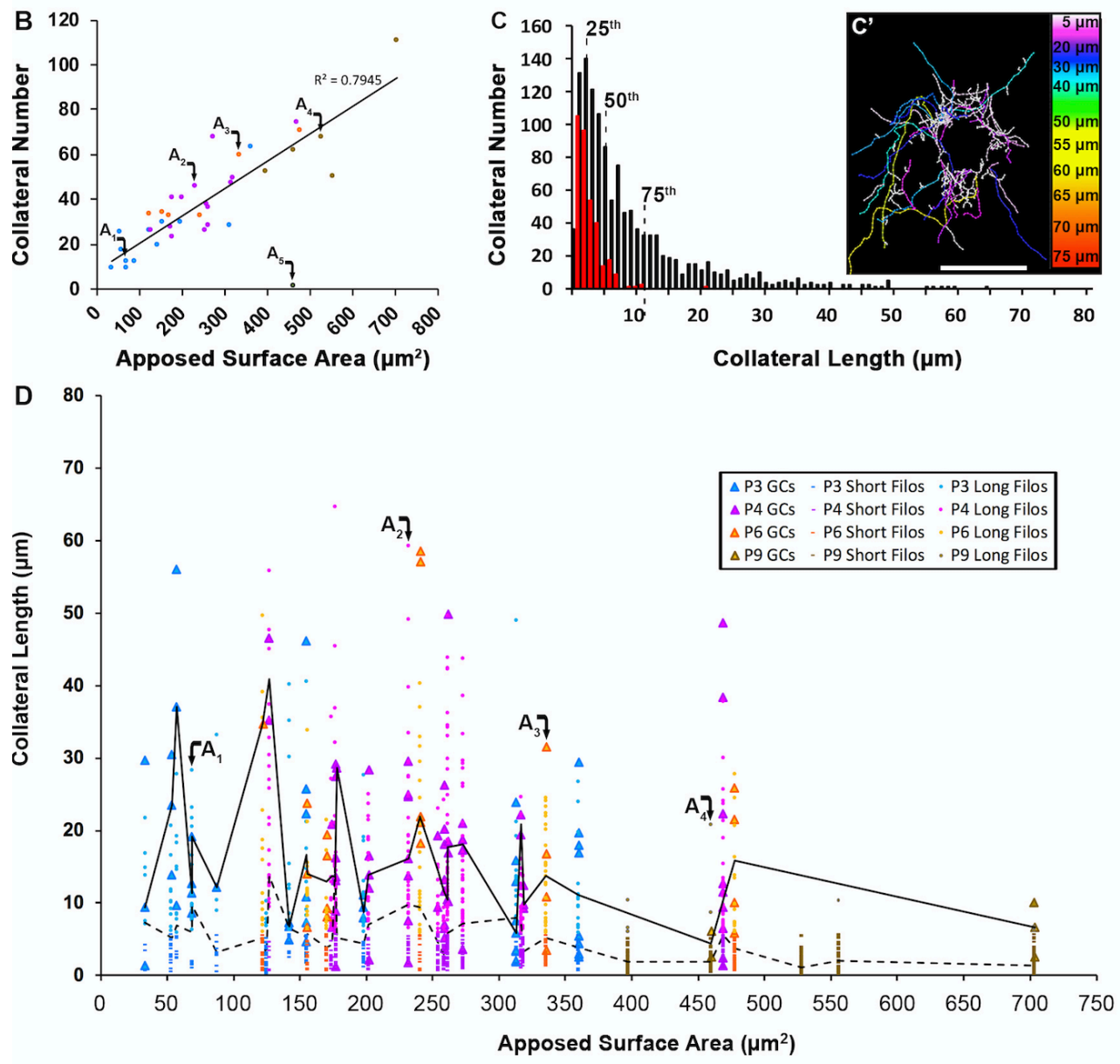

Chapter 2, Figure 1. Structural characterization of $\mathrm{CH}$ collateral network. A, Collaterals of CHs of different sizes and developmental stages. $\mathrm{A}_{1}-\mathrm{A}_{4}$, Short filopodia (chevrons) and longer filopodial (open arrowheads) and growth cone-tipped (closed arrowheads) thin collaterals extend in large numbers from CHs at neonatal ages. A5, Collaterals are largely absent by maturity, P30. $\mathbf{B}$, The absolute number of collaterals increases with $\mathrm{CH}$ size until P6; collaterals consolidate immediately prior to the onset of detection of peripheral auditory cues. $\mathbf{C}$, Distribution of collateral stalk length excluding added length of preterminal collateral arborization. Bin size: 1 $\mu \mathrm{m}$. Dotted gray lines: $25^{\text {th }}, 50^{\text {th }}$ and $75^{\text {th }}$ quartiles. Inset, Thin collaterals were skeletonized and their length measured from their origin at $\mathrm{CH}$ terminal to their distal tip. $\mathrm{CH}$ from $\mathrm{A}_{2}$. $\mathbf{D}$, Length of all collaterals fully contained within the SBEM volume plotted with respect to the ASA of each developing $\mathrm{CH}$. 50 ${ }^{\text {th }}$ percentiles indicated for long filopodial-tipped (circular symbols linked by black dashed lines) and growth cone-tipped collaterals (filled triangles linked by black solid lines) (color code referencing age matches scheme used in B). Scale bars: A, C, $20 \mu \mathrm{m}$. 
Figures

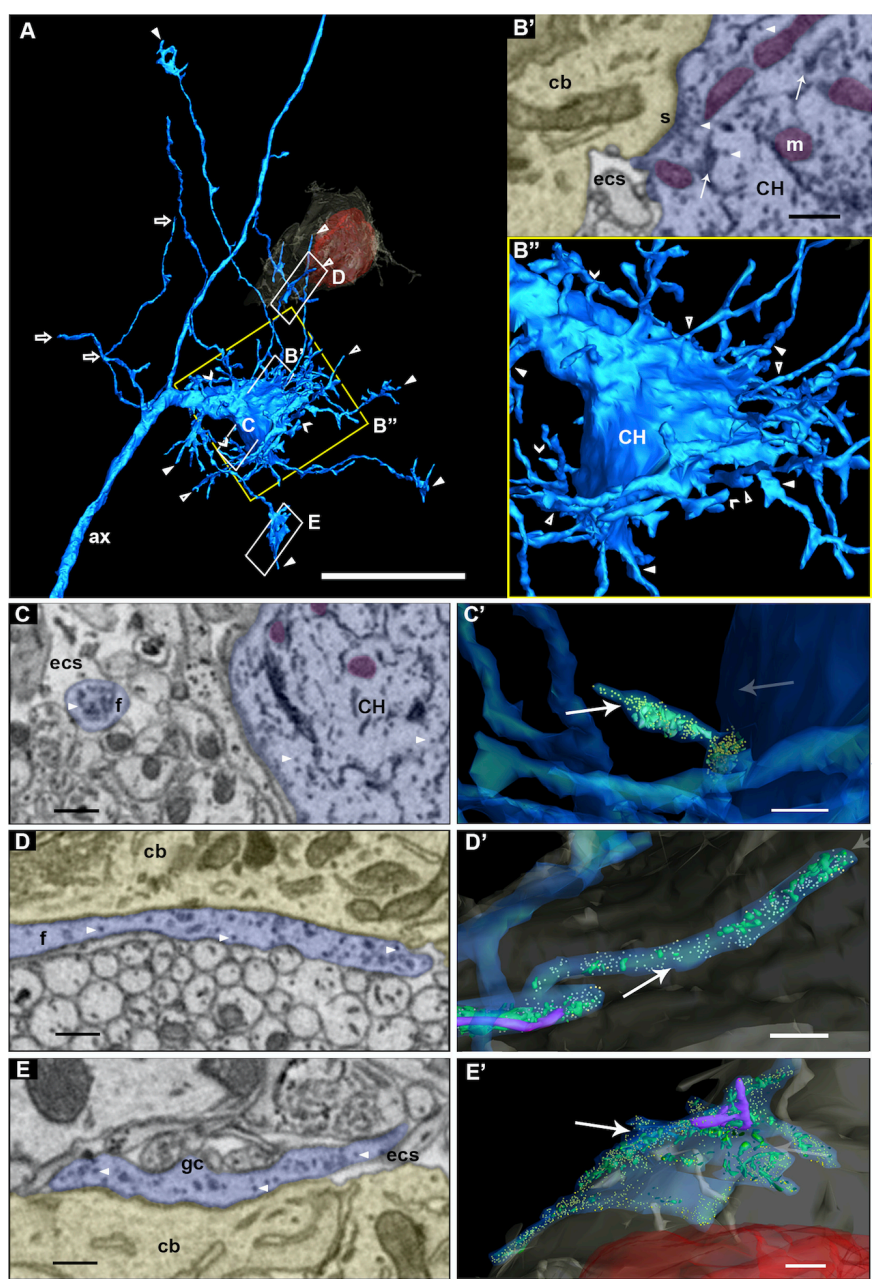

Chapter 2, Figure 2. Structural elements of the developing calyx of Held (CH). A, P3 calyx. Its afferent axon (ax) and smaller diameter distal axonal extension (ax') exit the image volume. Interstitial branches of different lengths (open arrows) emerge from the axon. Three categories of thin collaterals extend from the $\mathrm{CH}$ : short neurites with filopodial terminations (two examples, chevrons), long neurites with blunt filopodial terminations (four examples, open arrowheads), and long neurites with growth cone terminations (five examples, filled arrowheads). Only one of four different MNTB neurons innervated by this $\mathrm{CH}$ is depicted. Inset, Close-up of yellow boxed area reveals most collaterals emerging from the edges of the $\mathrm{CH}$. Symbols indicate origins of collaterals in A. B-E, Electron micrographs of regions boxed in panel A. cb, cell body of MNTB principal neuron; $\mathrm{m}$, mitochondria; s, synapse; white arrowhead, synaptic vesicle; $\mathrm{CH}$, calyx of Held; gc, growth cone; arrows, reticulated membrane; f, filopodia. B, CH apposed to cell body of MNTB principal neuron; presynaptic structures include mitochondria and synaptic vesicles clustered that near a convexity in the somatic membrane, forming a likely synapse. C, Growth cone-tipped collateral that does not contact a neuron has little intracellular structure except for sparse distribution of synaptic vesicles. D, Distal section through a filopodia-collateral where it apposes the cell body of the principal cell and is filled with synaptic vesicles. E, Proximal and distal sections through a growth cone-tipped collateral. In this case the growth cone extends in apposition to cell body and contains synaptic vesicles but lacks mitochondria. Scale bars: A, 20 $\mu \mathrm{m}$; B-E, $0.5 \mu \mathrm{m}$. 
Figures
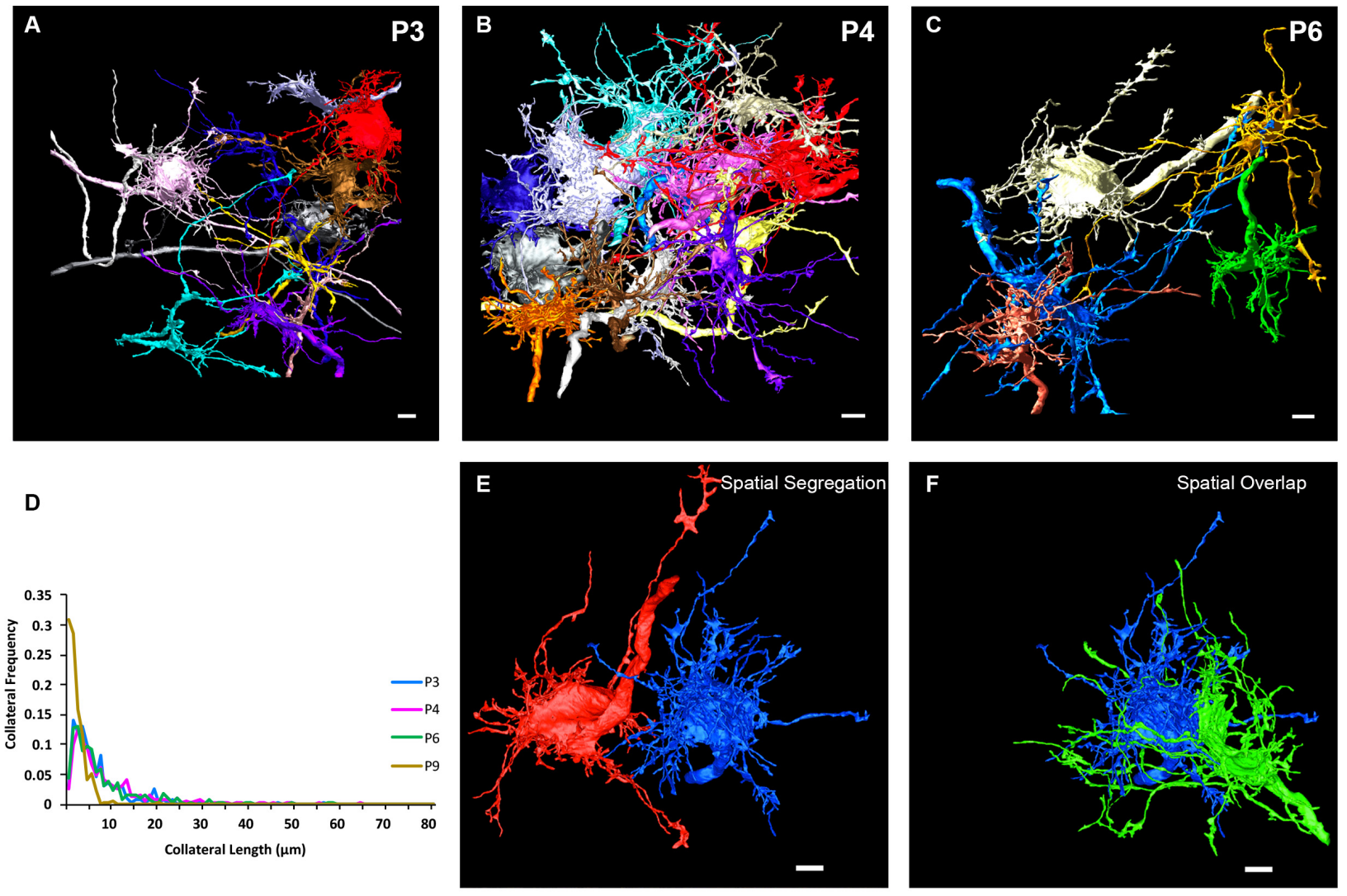

Chapter 2, Figure 3. Interactions between collaterals originating from different terminals. A-C, During early postnatal development $\mathrm{CH}$ terminals growing along nearby principal neurons extend collaterals within a common neuropil. D, Most collateral extensions emanating from $\mathrm{CH}$ terminals reside within a local proximity of $<10 \mu \mathrm{m}$ from the somatic surface of the principal cell being innervated. E, Neighboring principal cells may support $\mathrm{CHs}$ that do not make direct contact along the surface of any collaterals. F, Other neighboring pairs intimately intertwine within a shared volume between innervated neurons. Scale Bar: $5 \mu \mathrm{m}$. 

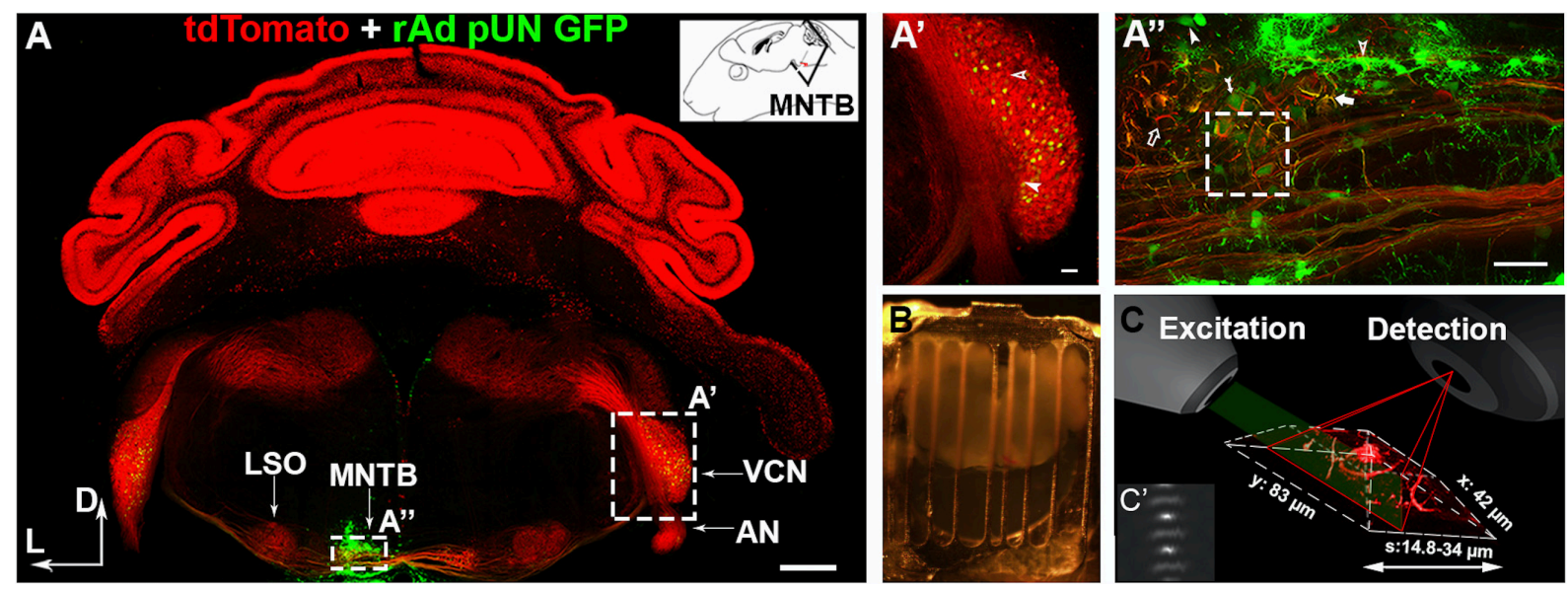

Chapter 2, Figure 4. Methods for tissue labeling and fluorescence imaging in acute coronal slices through the brainstem. A. P5 acute brain slice. Synaptic connections from the ventral cochlear nucleus (VCN) to the contralateral medial nucleus of the trapezoid body (MNTB) are largely preserved in a coronal slice through the auditory brainstem. Parvalbumin driven cre recombination leads to strong fluorescence (red, tdTomato) in the auditory nerve (AN), VCN cell bodies, $\mathrm{VCN}$ efferents projecting to the contralateral trapezoid body and presynaptic $\mathrm{CH}$ terminals within the MNTB. A subset of neurons and glia in the MNTB were labeled with EGFP (green) through recombinant adenovirus (rAd pUN GFP) introduced directly into the MNTB at P0. Inset: Slice plane. A', close-up of large rectangle in A: AN fibers and bushy cells within the VCN show tdTomato labeling. A subset of bushy cells were retrogradely labeled via the $\mathrm{CH}$ terminal by pUN EGFP virus (yellow; filled arrowheads). A", Expanded view of small rectangle in A: trapezoid body axons (red) and CHs (arrows) are strongly labeled with tdTomato. EGFP is expressed in a subset of principal neurons (fletched arrow), non-principal neurons (closed arrowhead) and glial cells (open arrowhead) within the MNTB. Box: approximate size of imaging field for lattice light sheet (LLS) microscopy. B, Tissue slices were embedded in 1\% low melting point agarose mounted on a slatted metal frame and subsequently affixed to a sample holder (labeled in D). C, Tissue positioned within a perfusion chamber and supplied with heated and oxygenated ACSF. The green plane indicates excitation sheet presentation through the sample orthogonally to the detection objective. The orthogonal arrangement of objectives results in collection of parallelepiped 4D volumes following each time point (arrow indicates direction in which sample is advanced; image volume presented at larger than actual scale relative to objectives). C', Custom Bessel patterns were optimized for imaging of fluorescent probes present in the sample (560 nm shown). 
Figures
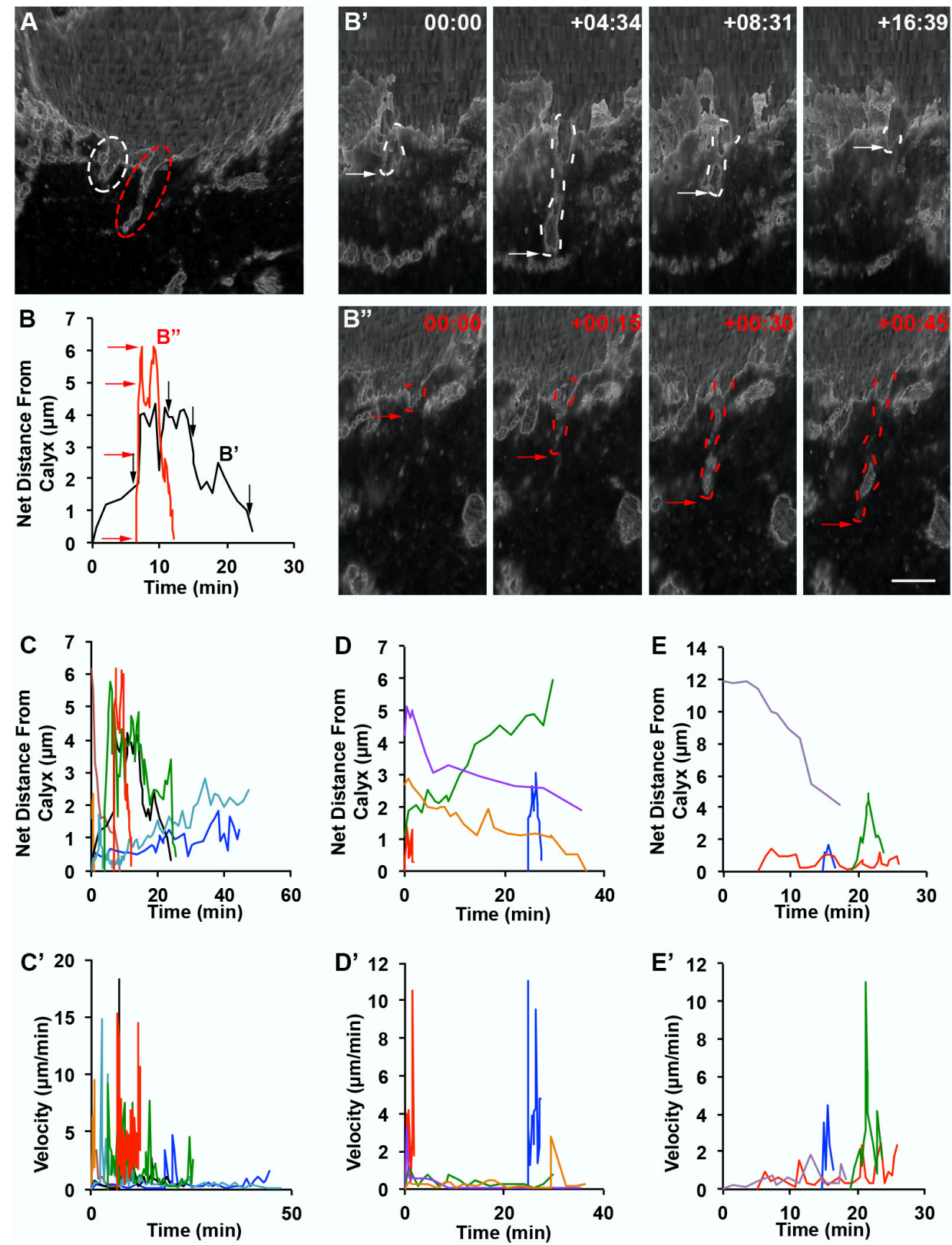

Chapter 2, Figure 5. Short collateral dynamics. A, Perspective view of labeled calyx shows presence of type I collateral branches. (Fluorescent signal presented with absorption model filter.) B-B" Manual tracking of the tip of individual collaterals reveal dynamic extension and retraction of each collateral outlined in A. Note a near synchronous extension and staggered retraction of each collateral. B'-B', Selected frames (correlated red and black arrows in B) depict length changes in two short collaterals from A. (Note a near synchronous extension and staggered retraction of each collateral.) C-E'. Upper panels $(\mathbf{C}, \mathbf{D}, \mathbf{E})$ described net changes in collateral length (each trace represents a unique collateral) as linearly defined as the distance from the $\mathrm{CH}$ to the tip of each collateral. Lower panels, $\left(\mathbf{C}^{\prime}, \mathbf{D}^{\prime}, \mathbf{E}^{\prime}\right)$ depict the rate of change in collateral trajectories observed during live imaging experiments. Scale Bar: B, $2 \mu \mathrm{m}$. 
Figures
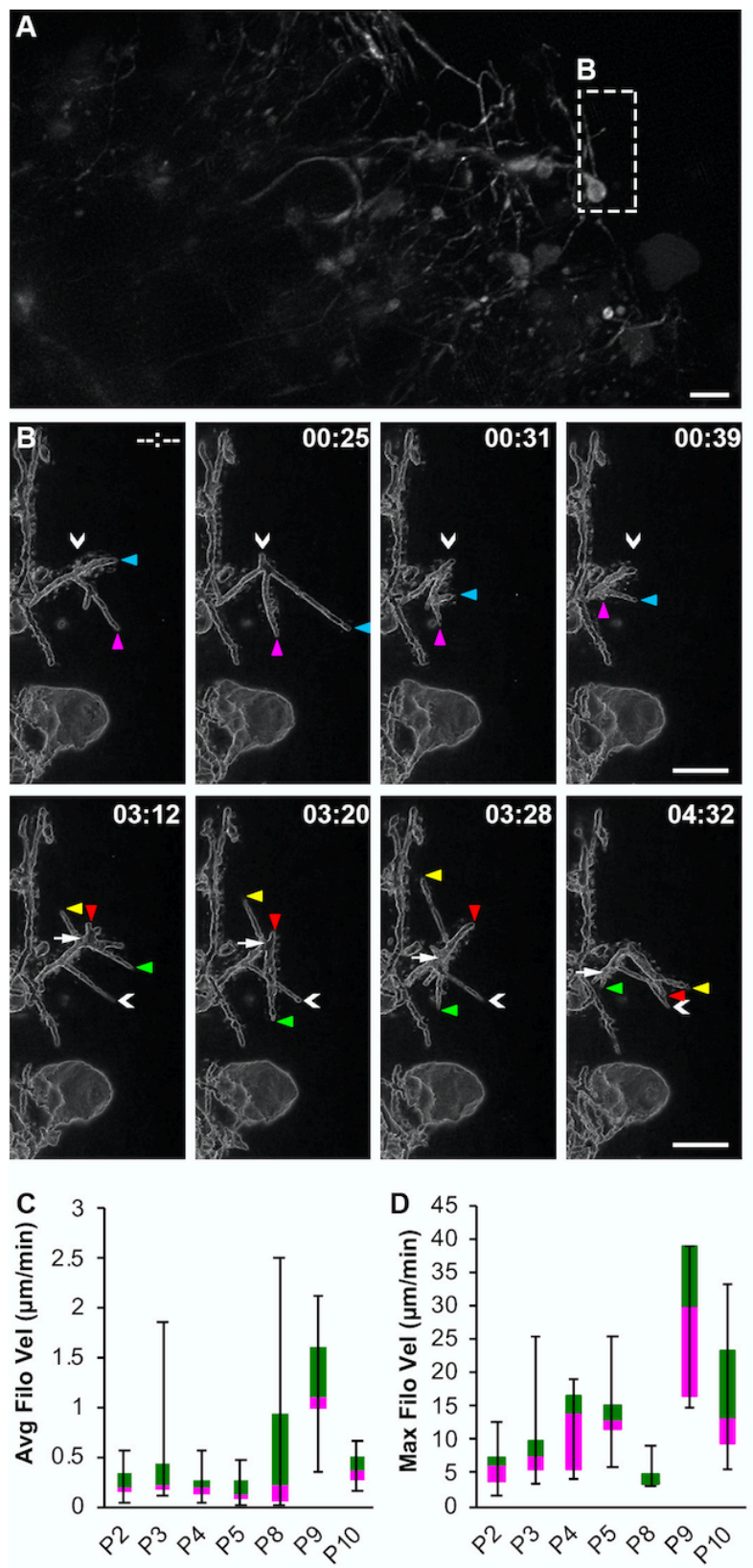

Chapter 2, Figure 6. Long filopodial collateral dynamics. A, Small volumes within the MNTB are dominated by fluorescently labeled $\mathrm{CH}$ collaterals. B, Selected frames from a P3 tissue section highlight the emergence, translocation and retraction of several filopodial arbors emanating from the leading segment of a filopodial collateral branch. (sub volume from A). C-D, Average and maximum velocities of filopodia-tipped collaterals demonstrate reduced speeds relative to growth cone-tipped collaterals but surprising increase in motility as long collaterals begin to retract just prior to the onset of hearing (P9). Scale Bar: $5 \mu \mathrm{m}$. 
Figures
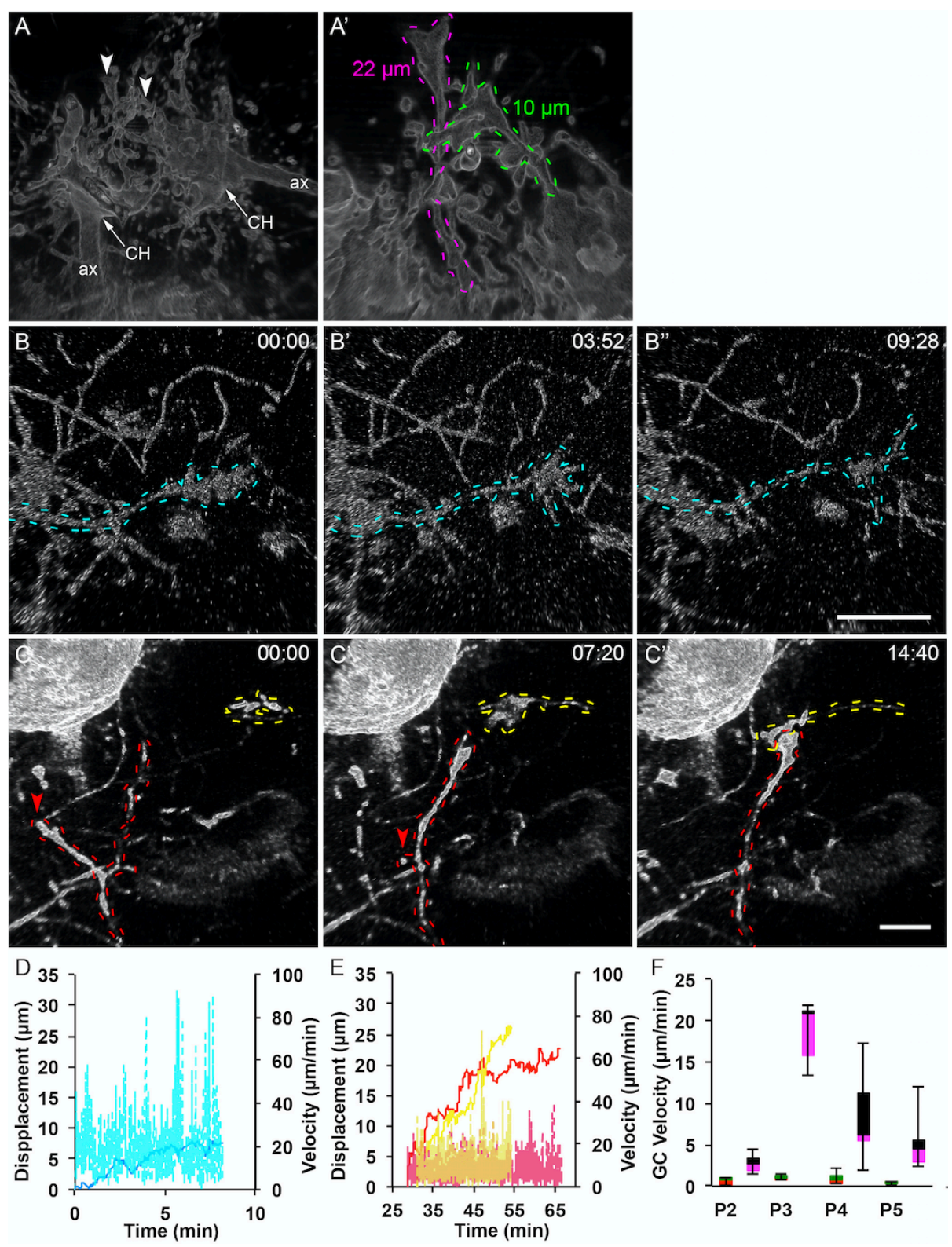

Chapter 2, Figure 7. Type III collateral dynamics. A, Perspective view of two labeled CHs exhibit intertwined collateral networks. Note at least two type III collaterals in the center of the frame (arrowheads). (Fluorescent signal presented with absorption model filter.) A', Zoomed view from A provides clearer depiction of growth cones emanating tens of microns from their CHs or origin. B, Time lapse images from P3 reveal motility of type III collateral (cyan). C', Time lapse images from P5 reveal similar motility of type III collaterals (red and yellow). D, Plot of the velocity (dotted) and displacement (velocity) versus time of the type III collateral in B. Peak velocities for this growth cone reached above $80 \mu \mathrm{m} / \mathrm{min}$ with sampling frequency of 1.3 Hz. E, Plot of the velocity (dotted) and displacement (velocity) versus time of the type III collaterals in $\mathbf{C}$. Peak velocities for this growth were generally less than those found at P3. Note, long branch retracted between frames (arrow). F, Average (red/green) versus maximum (pink/black) velocities reveal differences in motility across age. The fastest movement of type II collaterals occurred at P3. Scale Bars: B,C, $10 \mu \mathrm{m}$ 
Figures

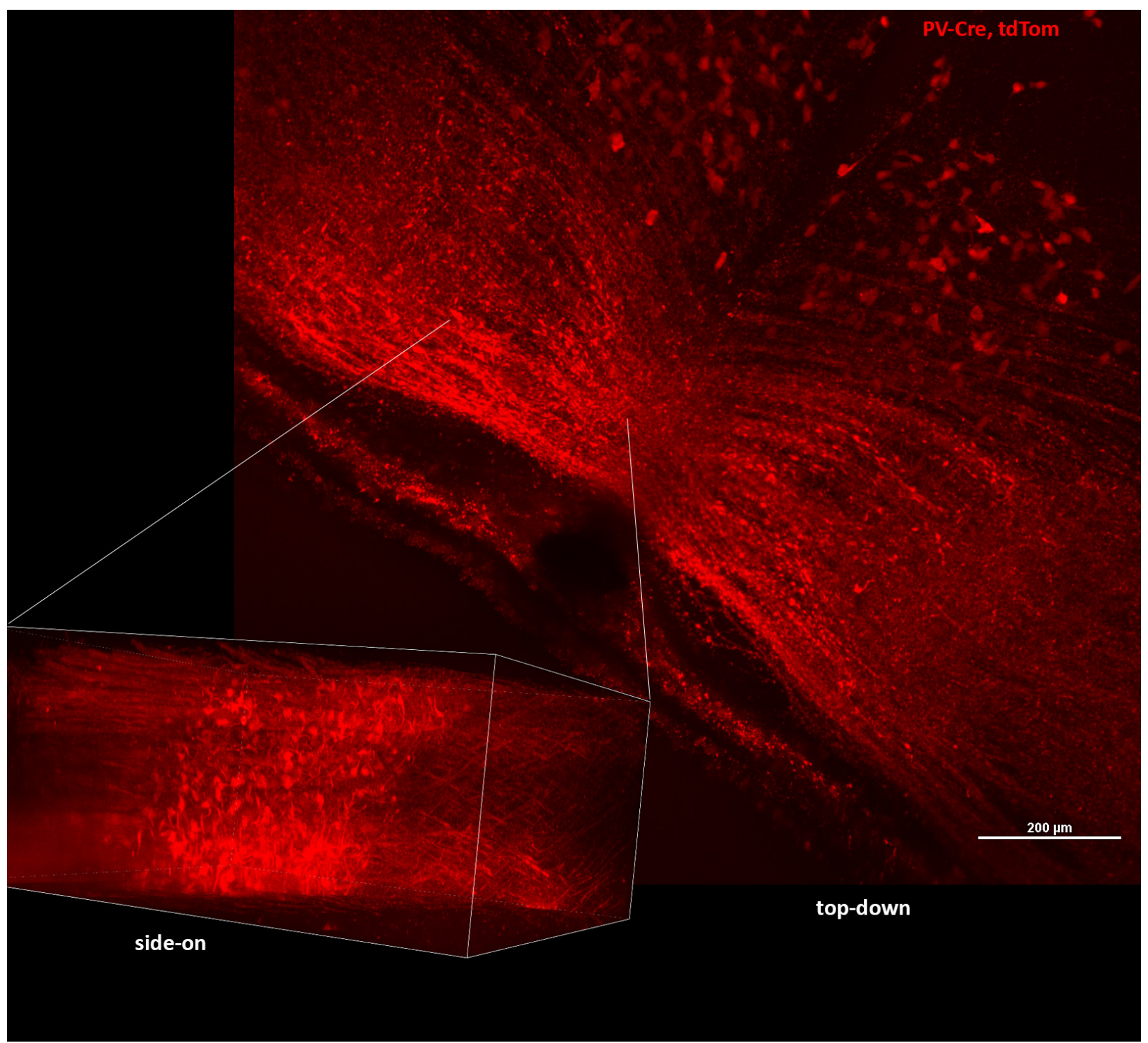

Chapter 2, Supplemental Figure 1. PV-cre driven expression of tdTom in the ventral auditory brainstem. Top-down view of thick coronal section $(300 \mu \mathrm{m})$ sectioned in the coronal plane imaged with two photon microscopy. Inset, Rotated view about the MNTB. Scale Bars: $200 \mu \mathrm{m}$ 

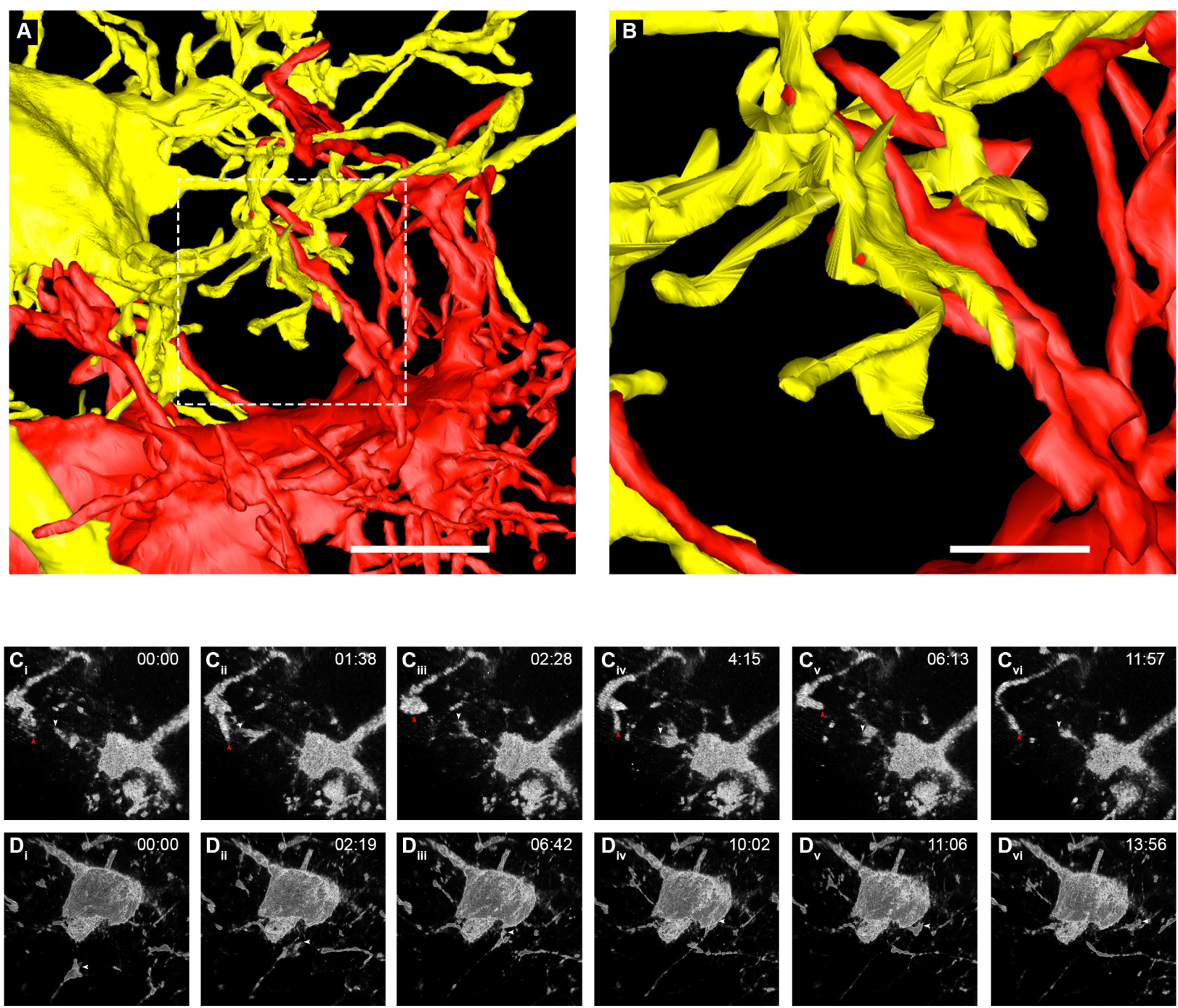

Chapter 2, Supplemental Figure 2. Neighboring $\mathrm{CH}$ terminals make frequent contact. A, Two neighboring $\mathrm{CHs}$ that innervate adject PCs exhibit extensive overlap of collaterals. B, A closer inspection reveals a complex association between arbors arising from different cells. $\mathbf{C}$, Live LLS time-lapse reveals contact between growth cones is transient ( $<30$ seconds). D, Growth cones were similarly observed contact the spoon like terminal growing along a less immediate neighbors $(>20 \mu \mathrm{m})$. Scale bars: $\mathbf{A}, 5 \mu \mathrm{m} ; \mathbf{B}, 2 \mu \mathrm{m}$ 
Figures

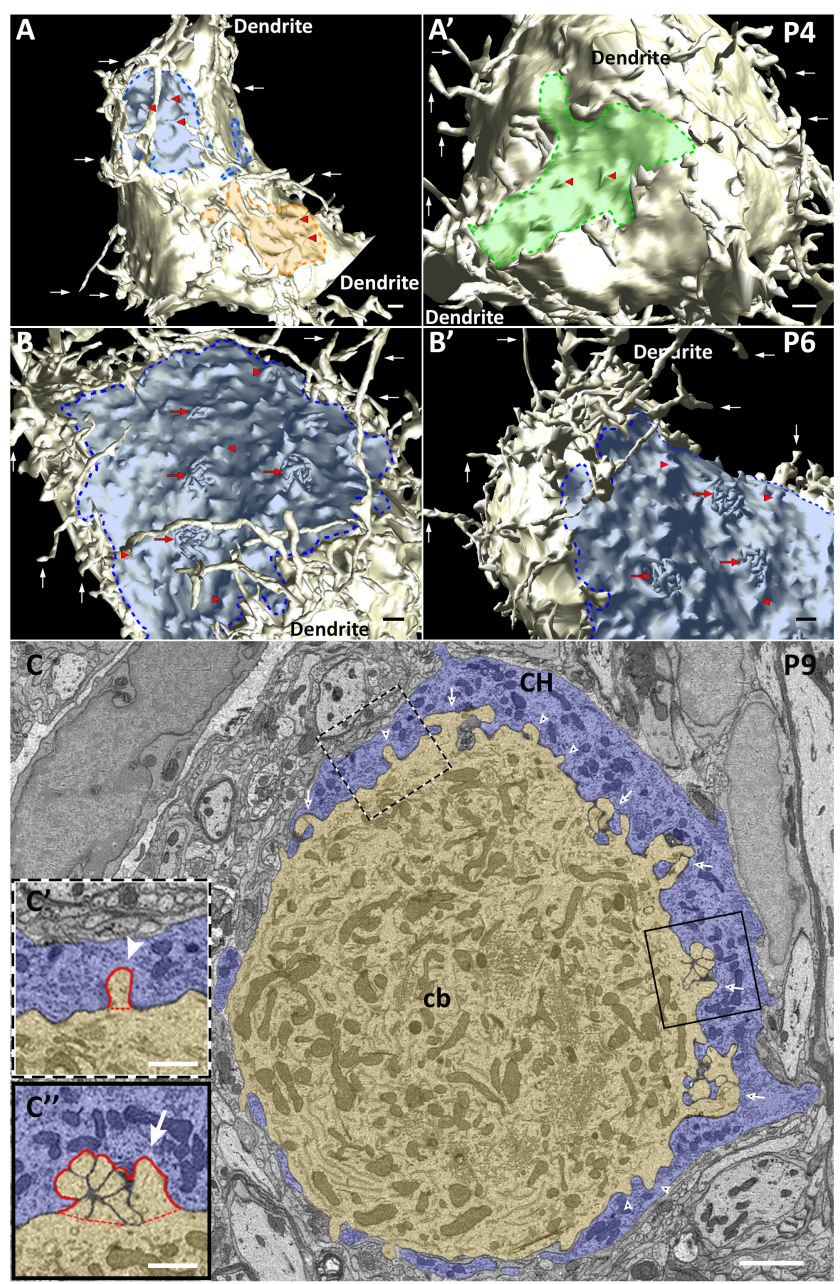

Chapter 3, Figure 1. Somatic processes within and peripheral to territory of calyx of Held $(\mathrm{CH})$ nerve terminals. A-A', 3D reconstructions reveal that multiple calyceal terminals (region of membrane apposition with cell soma outlined and shaded in blue, green and orange, in descending order of size respectively) converge on a single principal cell (PC) soma at P4. Single somatic processes, called type 1 spines, visualized as bumps on the somatic surface (examples indicated by red arrowheads), extend into a matching invagination within the presynaptic face of the terminal (not shown). Long, branched somatic appendages (white arrows) extend from the somatic surface outside of the borders of each growing calyx. B-B', Somatic processes that underlie larger $\mathrm{CH}$ terminals occur singly (type 1 spines, red arrowheads) or intertwined into spine mats (red arrows). Many of the longest somatic processes occur at the edge of the calyx apposition territory (blue). C, Single electron micrograph reveals the $\mathrm{CH}$ nerve terminal (labeled $\mathrm{CH}$ and shaded blue) contacting the cell body (cb, gold) of a P6 principal neuron. Numerous type 1 spines (white arrowheads) and spine mats (white arrows) extend into the $\mathrm{CH}$. C'-C', Boxed areas are expanded to show a single somatic type 1 spine ( $\mathrm{C}^{\prime}$; dotted inset) and outline the component spines, called type 2 spines, that form a spine mat (C"; solid inset). Red traces indicate the borders of each spine structure as traced across serial sections for subsequent 3D meshing and quantification. Scale Bar: A-B', $1 \mu \mathrm{m} ; \mathbf{C}, 2 \mu \mathrm{m} ; \mathbf{C}^{\prime}-\mathbf{C}^{\prime}, 0.5 \mu \mathrm{m}$. 
Figures
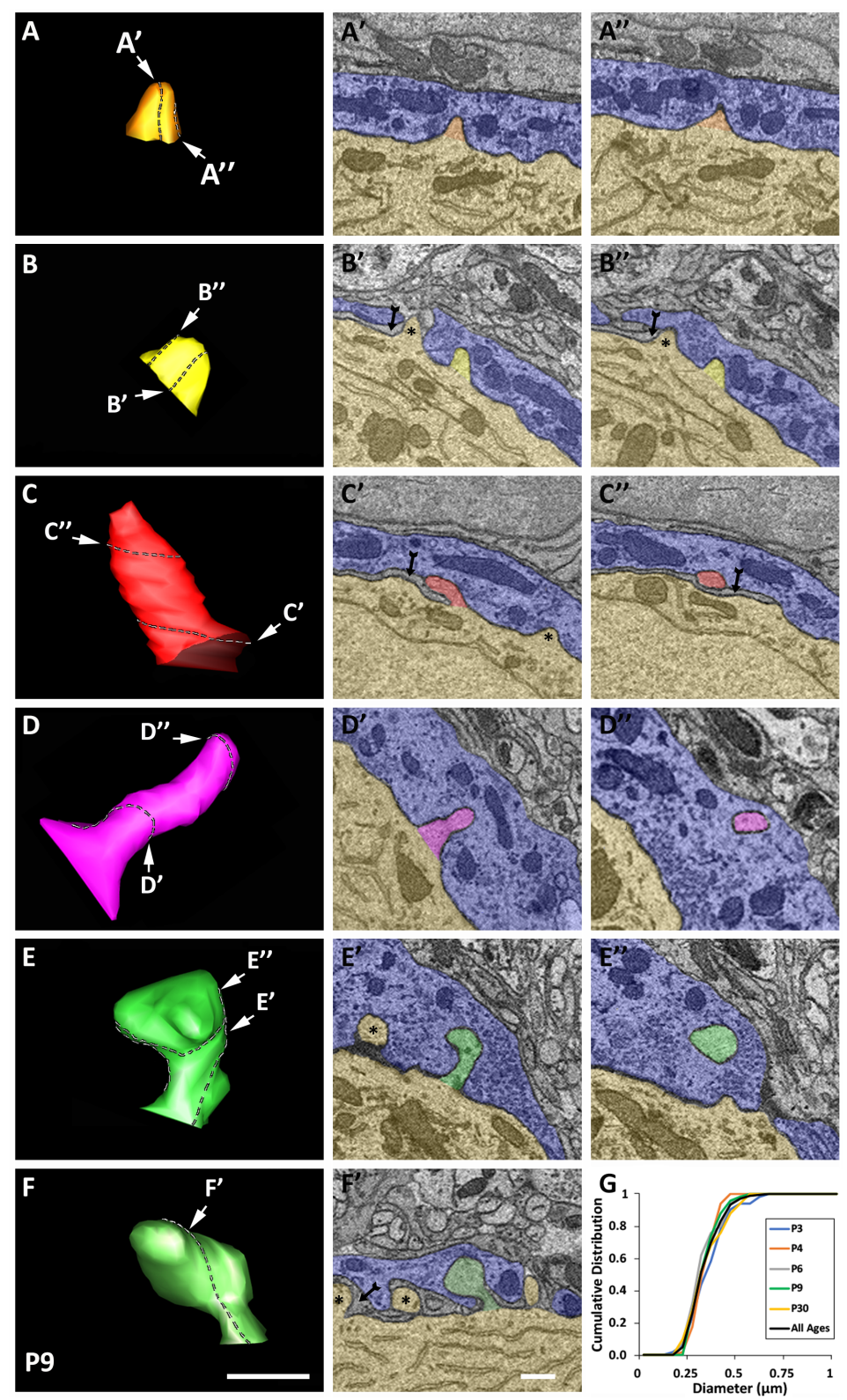

Chapter 3, Figure 2. Varying morphology of somatic type 1 spines from principal neurons. AG, 3D reconstructions (left column) and two image planes (middle and right columns; image planes indicated by dashed lines on 3D structure) through each type 1 spine. A-B. Short type 1 spines have similar base (A" and B") and length dimensions. In B', B', asterisk indicates nearby type 1 spine near the edge of the $\mathrm{CH}$ that contacts an astrocytic process (solid arrow). C-D. Long tubular type 1 spines could extend tangentially $\left(\mathbf{C}^{\prime}, \mathbf{C}^{\prime \prime}\right)$ ) or through nearly the entire thickness of the calyx (D', D'), which can exceed $2 \mu \mathrm{m}$. Spine in $\mathbf{C}$ is also at edge of apposed surface area and contacts glial process (solid arrow). E-F. The most complex type 1 spine geometries include expansion and bending of the spine head. Asterisks indicate nearby type 1 spines. G, Despite heterogeneity in overall type 1 spine morphology, spine caliber remained consistent with $96 \%$ of type 1 spines maintaining a diameter between 0.2-0.5 $\mu \mathrm{m}(\mathrm{P} 3: 340 \pm 100 \mathrm{~nm} ; \mathrm{P} 4: 320 \pm 60 \mathrm{~nm}$; P6: $300 \pm 90$ nm; P9: $310 \pm 80$ nm; P30: $320 \pm 100$ nm). Scale Bar: A-F', $0.5 \mu \mathrm{m}$. 

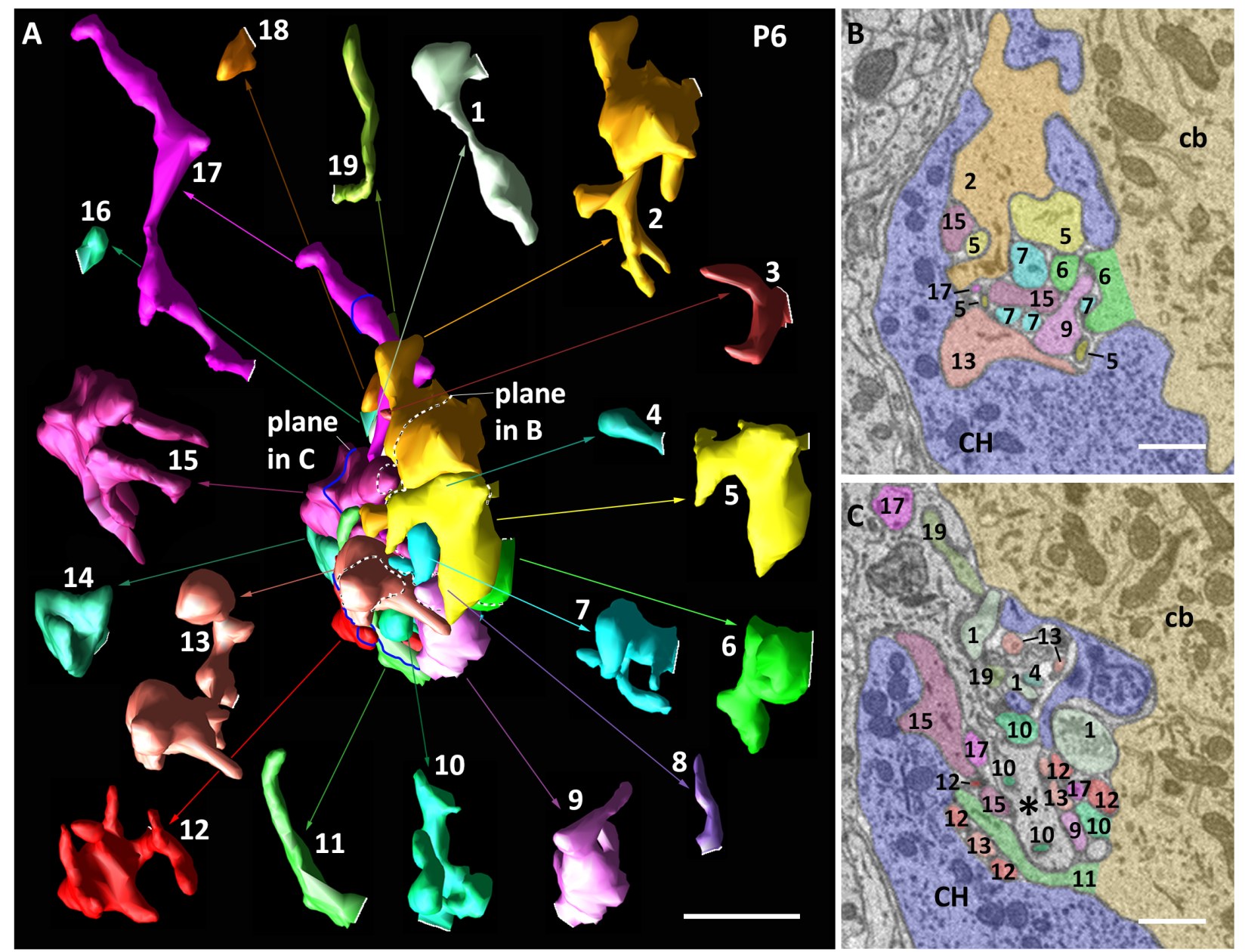

Chapter 3, Figure 3. Spine mat comprised of 19 type 2 spines from a P6 principal neuron. A, $3 \mathrm{D}$ reconstruction of spine mat, with individual type 2 spines shown in different colors. Each spine also shown in exploded view surrounding the spine mat. Individual type 2 spines can branch and generally exhibit more complex geometry (e.g., \#2, 5, 12, 15, 17) than type 1 spines. White line on each spine indicates location of attachment to the principal cell body. B-C, Single EM sections at two locations through the spine mat illustrate complex intertwining of 15 of the 19 type 2 spines. Colors and numerical labels correspond to the 3D reconstructions illustrated in A. Note in addition to various spines, both presynaptic calyceal processes (blue) and glial processes (asterisk, not colored) intermingle within spine mats. Scale Bar: A, $2 \mu \mathrm{m} ; \mathbf{B}_{\mathbf{i}}-\mathbf{B}_{\mathrm{ii}}, 1 \mu \mathrm{m}$. 
Figures
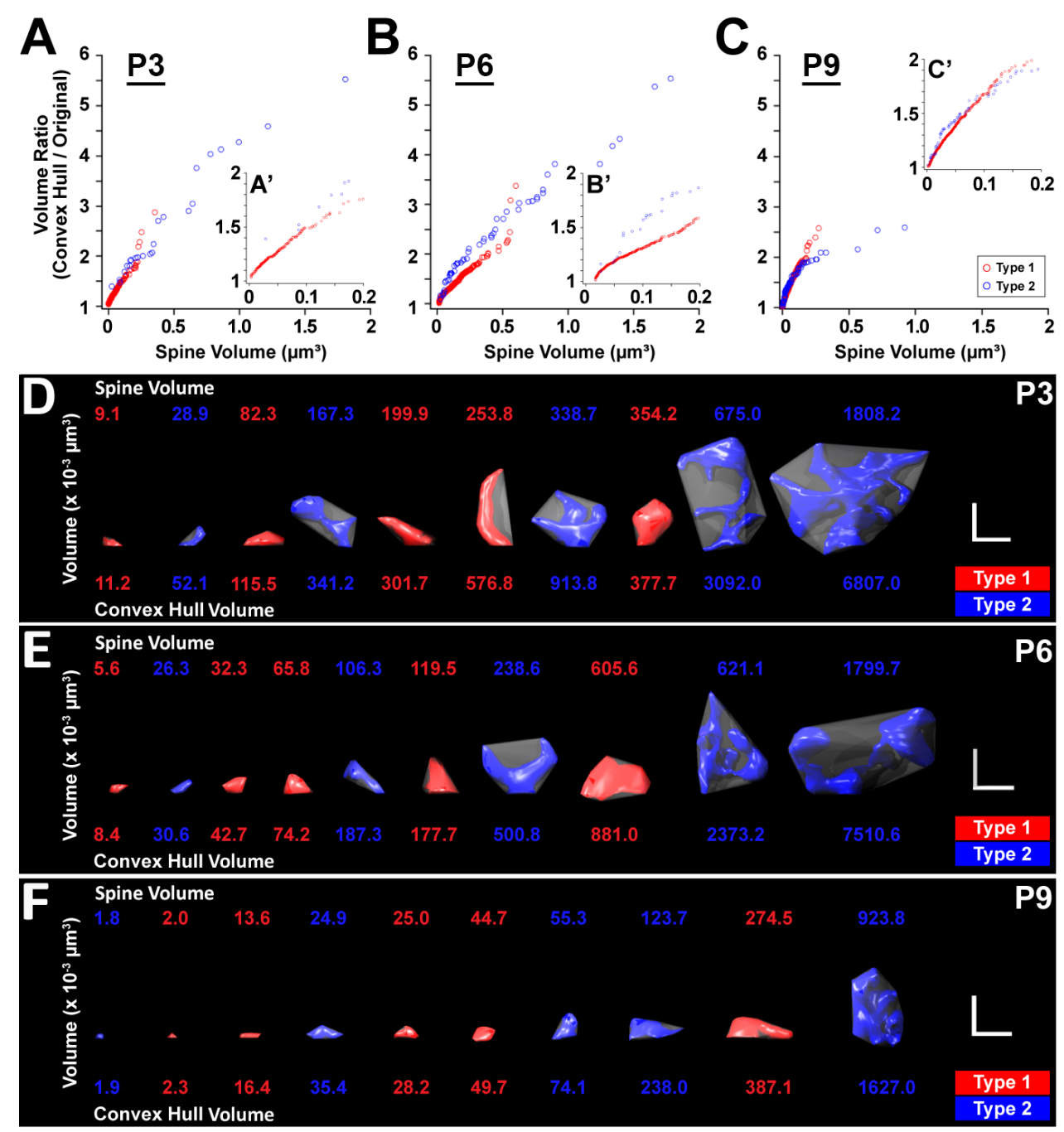

Chapter 3, Figure 4. Analysis of Spine Complexity using Convex Hull Fitting. A-C, The ratio of convex hull to corresponding spine mesh volume was calculated for type 1 (blue) and type 2 (red) spines and plotted against the spine volume across ages (P3,6,9). With the exception of a small number of cases for type 1 spines which represent highly curved fingerlike projections that lack true complexity (see panel $\mathbf{D}, 6^{\text {th }}$ spine from the left for an example), type 2 spines consistently showed greater complexity scores than type 1 spines at P3 and P6. Insets (A'-C') zoom into smaller values, where the majority of the data are located, reveals divergence of values above small volumes indicative of simple spines $\left(<0.2 \mu \mathrm{m}^{3}\right)$. Note that at P9 relatively fewer type 2 spines exceed $0.5 \mu \mathrm{m}^{3}$. (D-E) Representative type 1 (blue) and type 2 (red) spines encompassing the range of sizes for each at the three ages analyzed $(\mathrm{P} 3,6,9)$ are presented in order of increasing size (spine volumes above). Convex hulls (grey veils surrounding spines) were included for each object and in every case, produced larger resultant volumes (below). Based on these differences in size range and complexity difference of small spines, there was a statistically significant difference between single spines and intertwined spines at all ages (average complexity score for single vs. intertwined spines - P3: $1.32 \pm 0.3$ vs. $2.64 \pm 1.1$, P6: $1.31 \pm 0.3$ vs. $2.50 \pm 1.4$, P9: $1.27 \pm 0.2$ vs $1.52 \pm 0.3 ; \mathrm{p}<0.0001$ for all comparisons within age using the Mann-Whitney U-test (M-W); Fig. 4A-C). Scale bar: D-F, $1 \mu \mathrm{m}$. 
Figures

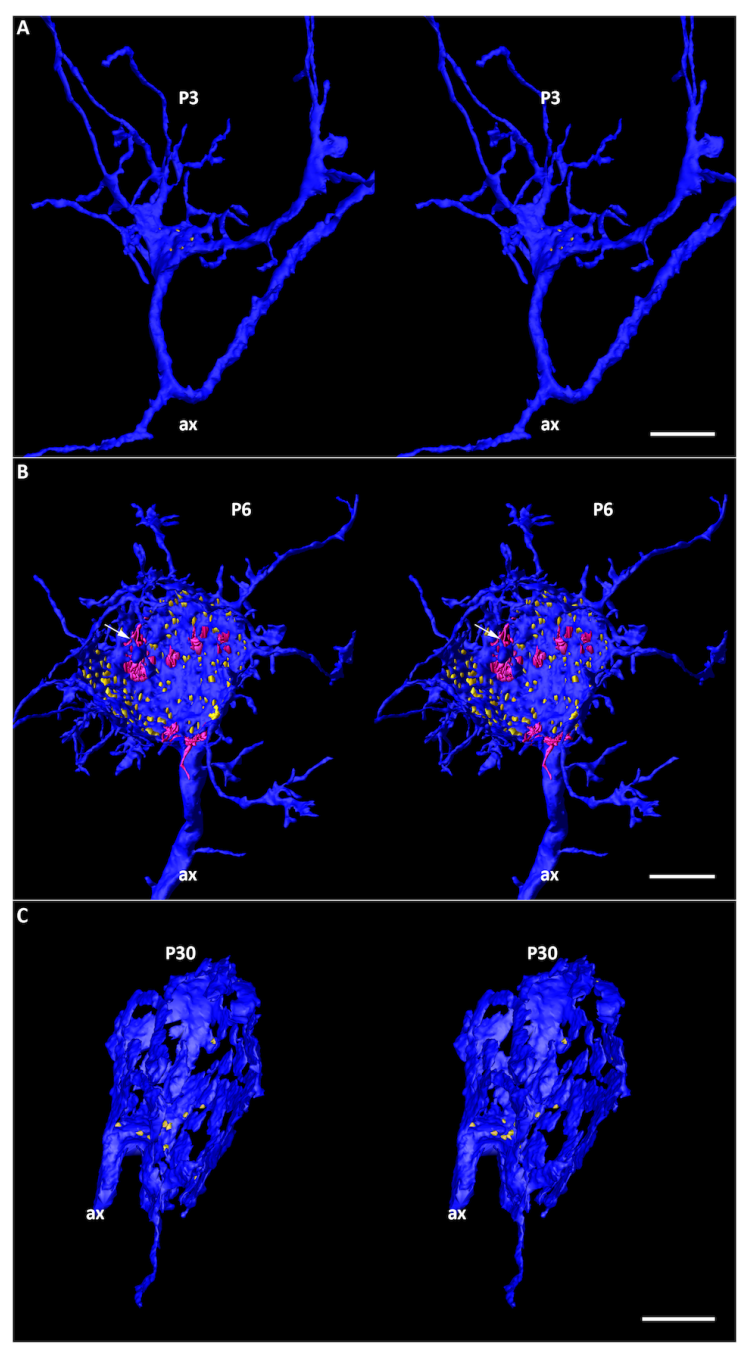

Chapter 3, Figure 5. Stereo views of single $\mathrm{CH}$ terminals oriented en face illustrate agedependent changes in type 1 spine and spine mat density during development of the $\mathrm{CH}$. $\mathbf{A}$, Large terminals ( $>25 \mu \mathrm{m}^{2}$ in ASA) identified as early as P3, reveal relatively few invaginations associated with somatic type 1 spines (yellow). B, By P6, larger densities of type 1 spines (yellow) and spine mats (assorted colors) are found throughout the area of pre- and postsynaptic association. Arrow in $\mathbf{B}$ corresponds to the spine mat presented in Figure 3. $\mathbf{C}$, The mature $\mathrm{CH}$ exhibits greater fenestration than immature terminals, a much lesser density of type 1 spines, and only rare occurrences of spine mats (none occur in this terminal). Mature $\mathrm{CHs}$ extend further around the perimeter of principal cell bodies and partially obscure the view of the inner surface. Scale Bar: A-C, $5 \mu \mathrm{m}$. 

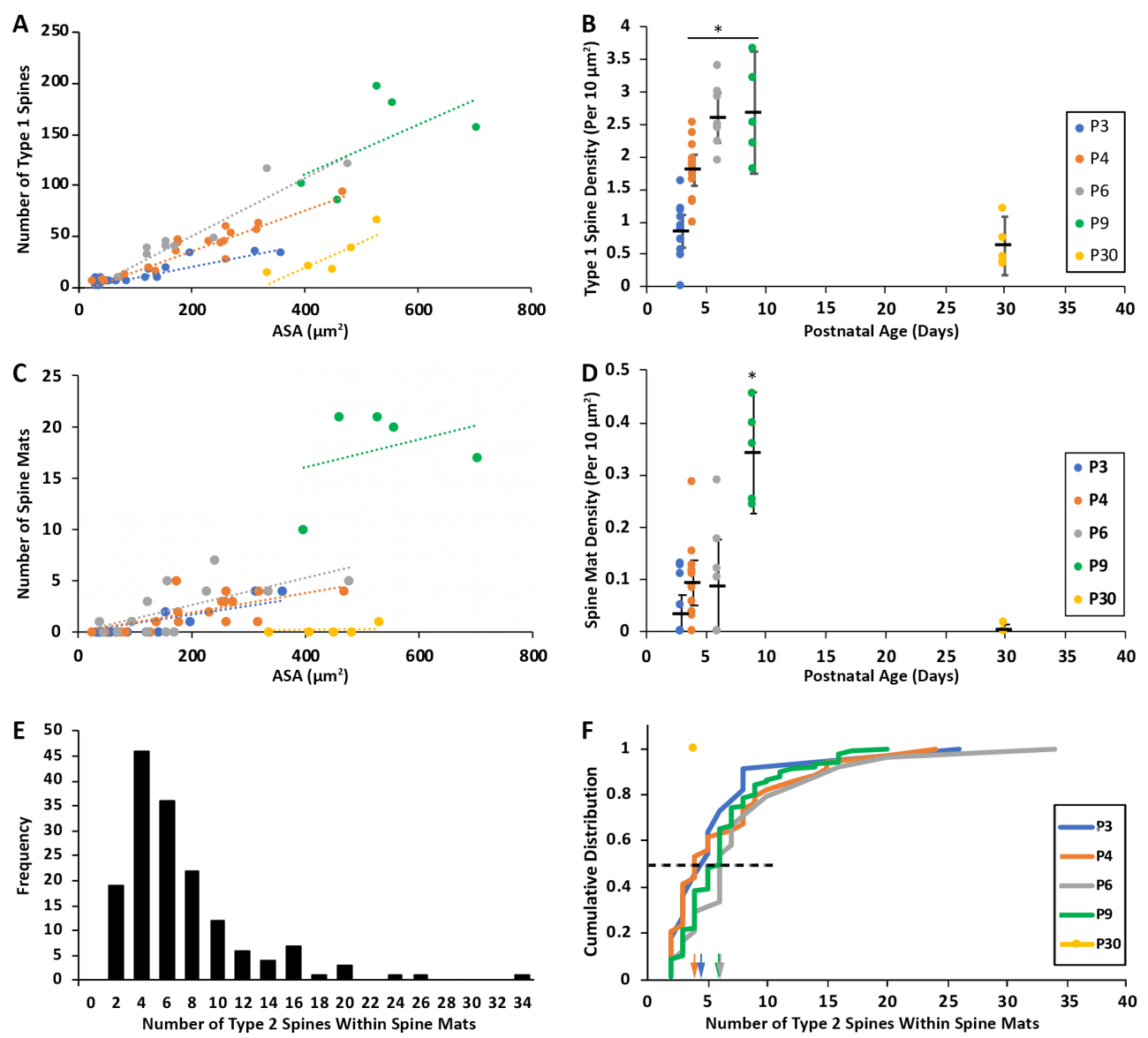

Chapter 3, Figure 6. Type 1 spine and spine mat occurrence and composition across developmental age. A, Number of type 1 spines increased with size of the calyx of Held $(\mathrm{CH})$. For a given terminal size, type 1 spine number increased with age from P3 to P6, the latter of which was similar to P9, and decreased at P30. Slope of line increased with age from P3 to P6 and did not change further through P30 $\left(\mathrm{P} 3, \mathrm{~m}=0.100, \mathrm{r}^{2}=0.92 ; \mathrm{P} 4, \mathrm{~m}=0.181, \mathrm{r}^{2}=0.96 ; \mathrm{P} 6, \mathrm{~m}\right.$ $\left.=0.267, \mathrm{r}^{2}=0.97 ; \mathrm{P} 9, \mathrm{~m}=0.268, \mathrm{r}^{2}=0.94 ; \mathrm{P} 30, \mathrm{~m}=0.243, \mathrm{r}^{2}=0.72\right) . \mathbf{B}$, Density of type 1 spines per $10 \mu \mathrm{m}^{2}$ of somatic surface area significantly increased between P3 and P9 $(\mathrm{p}<0.01, \mathrm{~K}$ $\mathrm{W})$ and returned to returned to initial densities at P30 similar to $\mathrm{P} 3(\mathrm{p}=0.99, \mathrm{~K}-\mathrm{W}) \mathbf{C}$, Spine mat number scaled similarly with size between P3-6, and the slope was similar but with larger yintercept at P9 $\left(\mathrm{P} 3, \mathrm{~m}=0.0086, \mathrm{r}^{2}=0.6828 ; \mathrm{P} 4, \mathrm{~m}=0.0095, \mathrm{r}^{2}=0.502 ; \mathrm{P} 6, \mathrm{~m}=0.0132, \mathrm{r}^{2}=\right.$ 0.4326; P9, $\left.\mathrm{m}=0.0136, \mathrm{r}^{2}=0.1133 ; \mathrm{P} 30, \mathrm{~m}=0.0005, \mathrm{r}^{2}=0.4527\right)$. Across age, $\mathrm{CHs}<150 \mu \mathrm{m}^{2}$ generally lacked spine mats, as did most cells at P30. D, Similar to type 1 spine density, spine mat density per $10 \mu \mathrm{m}^{2}$ of somatic surface area significantly increased between P3 and P9 $(p<0.01, K-W)$ before spine mats again become significantly rarer by maturity at $\mathrm{P} 30(\mathrm{p}<0.01$, $\mathrm{K}-\mathrm{W})$. E, Distribution for number of type 2 spines in each spine mat, combined across age because of similarity in distributions (mean: $6.8 \pm 5.0$; median: 6.0; mode: 4.0). F, Cumulative distribution of spine mat composition (as defined by the number of component type 2 spines) reveals the narrowest distribution at P3 and widest at P4, P6 and P9. At P30, only one spine mat was found. Dashed line illustrates the $50^{\text {th }}$ percentile in the cumulative distribution function and reveals a shift to more type 2 per mat between P6 and P9 (gray and green arrows, respectively) relative to younger ages. 
Figures

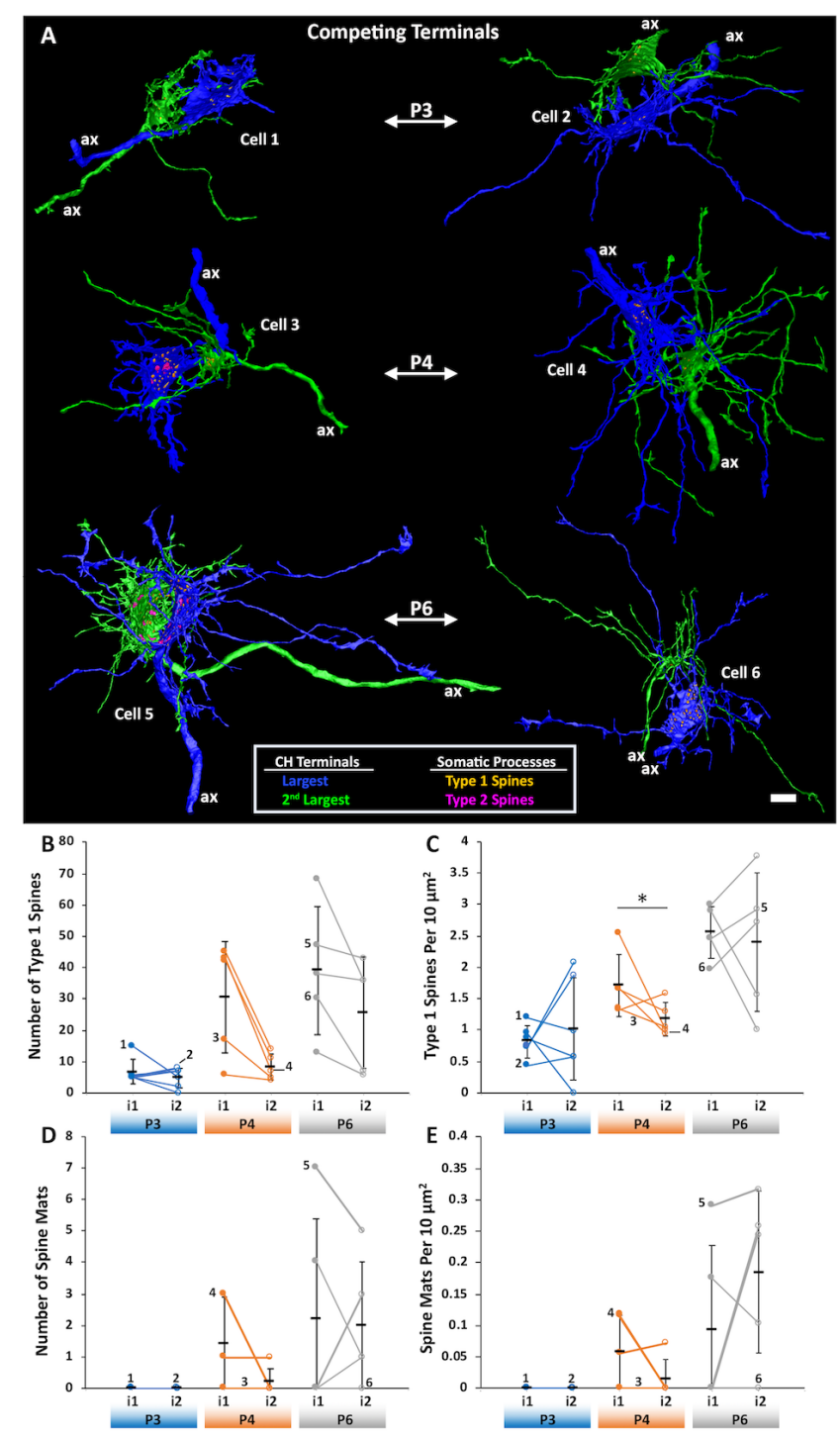

Chapter 3, Figure 7. Competition among $\mathrm{CH}$ terminals. A, Two representative examples of competing CHs with unique axons (ax) at P3, P4 and P6 (largest input labeled in blue, second largest input in green; as measured by ASA). Somatic spines (red) and spine mats (pink) situated within invaginations in the associated competing input are illustrated without the soma to reveal their locations across the presynaptic face of each terminal. B, The largest input of the competing pair tends to cover more type 1 spines. C, Type 1 spine density, however, is not consistently greater in the largest $\mathrm{CH}$ as only at $\mathrm{P} 4$ does the larger input contain significantly more embedded type 1 spines $(\mathrm{p}<0.05, \mathrm{~K}-\mathrm{W}$ with Nemenyi test $)$. D, E, No spine mats were found in competing terminals at P3. At P4 and P6 there is no consistent relationship between spine mat number or density and $\mathrm{CH}$ size. Scale Bar: A, $5 \mu \mathrm{m}$. 
Figures
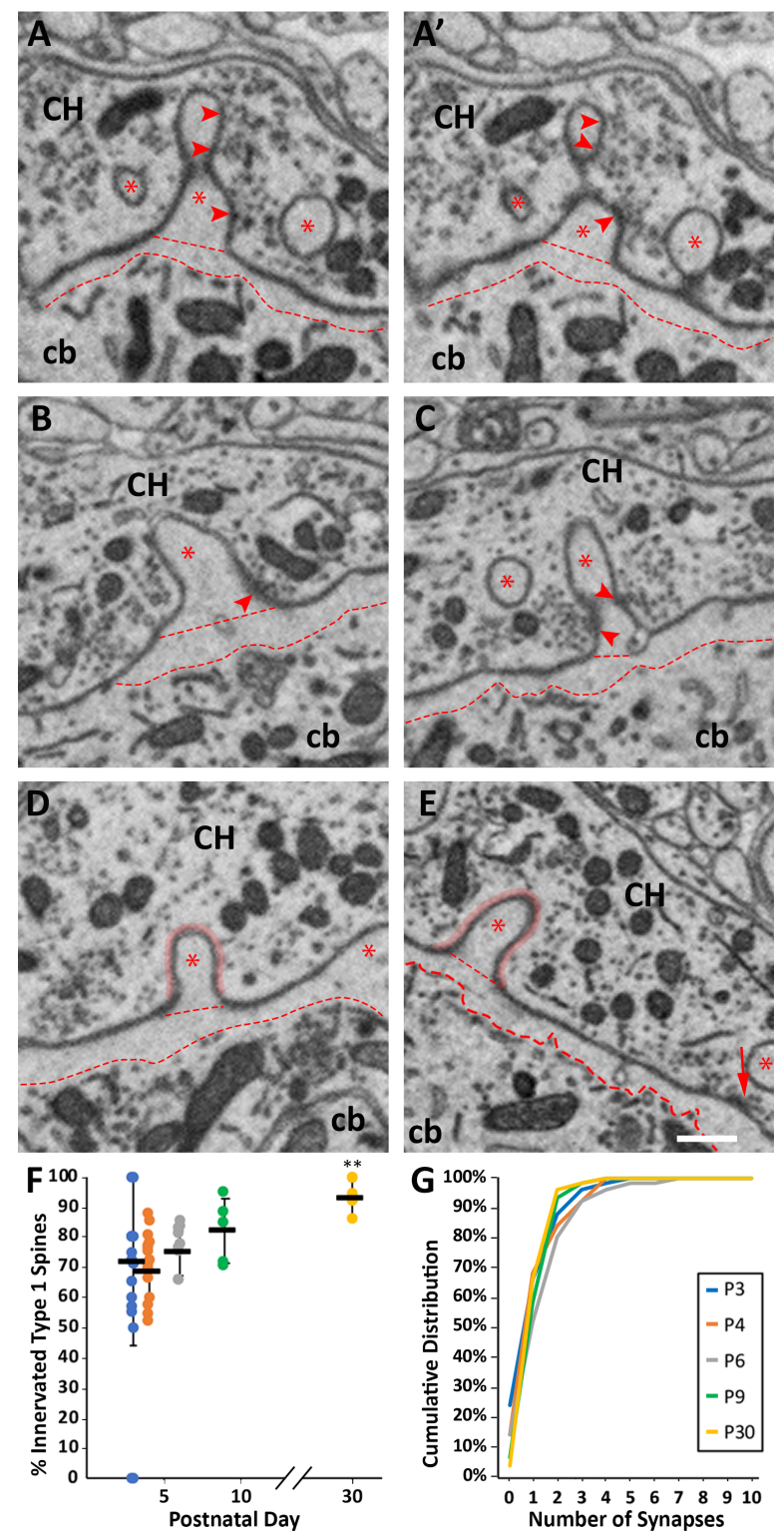

Chapter 3, Figure 8. Type 1 spine ultrastructure and innervation. A-E, Synapses (arrowheads) identified in SBEM images by dense clusters of vesicles near the presynaptic membrane across multiple sections and located adjacent to membrane in at least one section. Type 1 spines are largely devoid of stained organelles. This organelle exclusion zone extends several hundred $\mathrm{nm}$ into the cell body (dotted lines). Straight dotted lines indicate base of type 1 spines. A-A', Adjacent sections reveal synapses located at base (lowest arrowhead) and near tip of a single type 1 spine (middle and top arrowheads). Asterisks indicate nearby type 1 spines. B-C, Synapses were most frequently identified at the base of type 1 spines. D-E, A subset of type 1 spines contained no visible synapses and exhibited a thin layer (transparent red band) adjacent to the presynaptic membrane devoid of vesicles. Typical somatic synapses were used as a reference to characterize inputs to type 1 spines. cb, cell body; $\mathrm{CH}$, calyx of Held. F, Most type 1 spines were innervated throughout development with at least one visible synapse. By P30, nearly all type 1 spines were innervated. $\mathbf{G}$, Cumulative distribution functions show that multiple synapses could occur onto a single spine at each age. Scale Bar: A-D, $0.5 \mu \mathrm{m}$. 
Figures
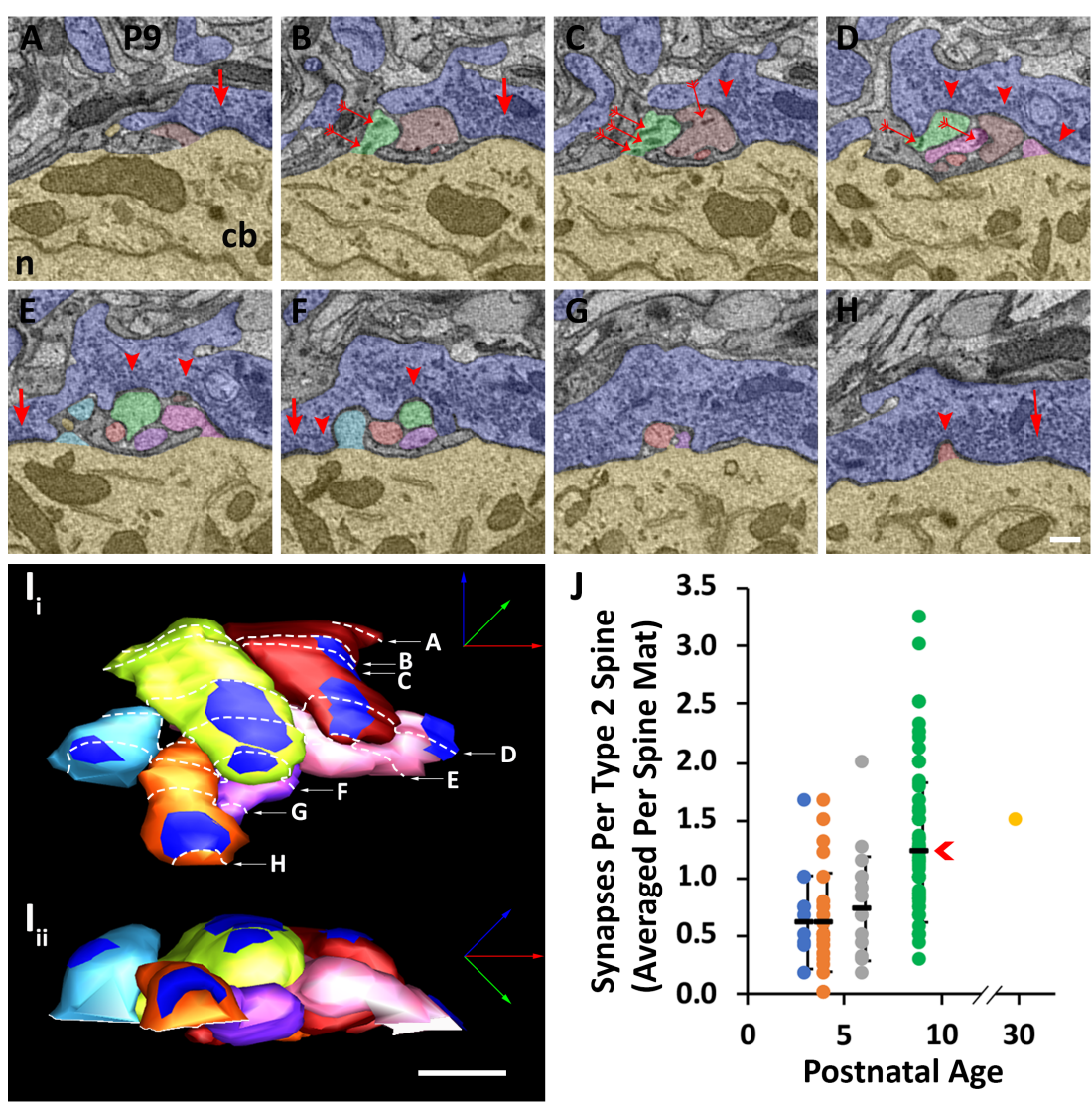

Chapter 3, Figure 9. Innervation of type 2 spines within spine mats. A-H, Synapses (arrowheads) found along type 2 spines were identified using the same criteria as type 1 spine inputs (red arrow indicates traditional somatic synapses along more flattened region of cell membrane for comparison). B-D, Postsynaptic organelles including reticulated membranous structures (fletched arrows) could infiltrate into type 2 spines. I, Three dimensional rendering of the spine mat segmented in A-H reveals the close association of type 2 spines typical of spine mats. Ii, The rendering above presents a top-down perspective looking through the presynaptic terminal to illustrate the occurrence of seven synapses found along the surface of the spine mat. Iii, $90^{\circ}$ rotation of the spine mat reveals the attachment site (solid white overlay) of three of the six type 2 spines of the spine mat. J, Synapse density onto type 2 spines is similar from P3-6 and increased at P9 (P9, p < 0.01, Pairwise MW). Scale Bar: A-I, $0.5 \mu \mathrm{m}$. 
Figures

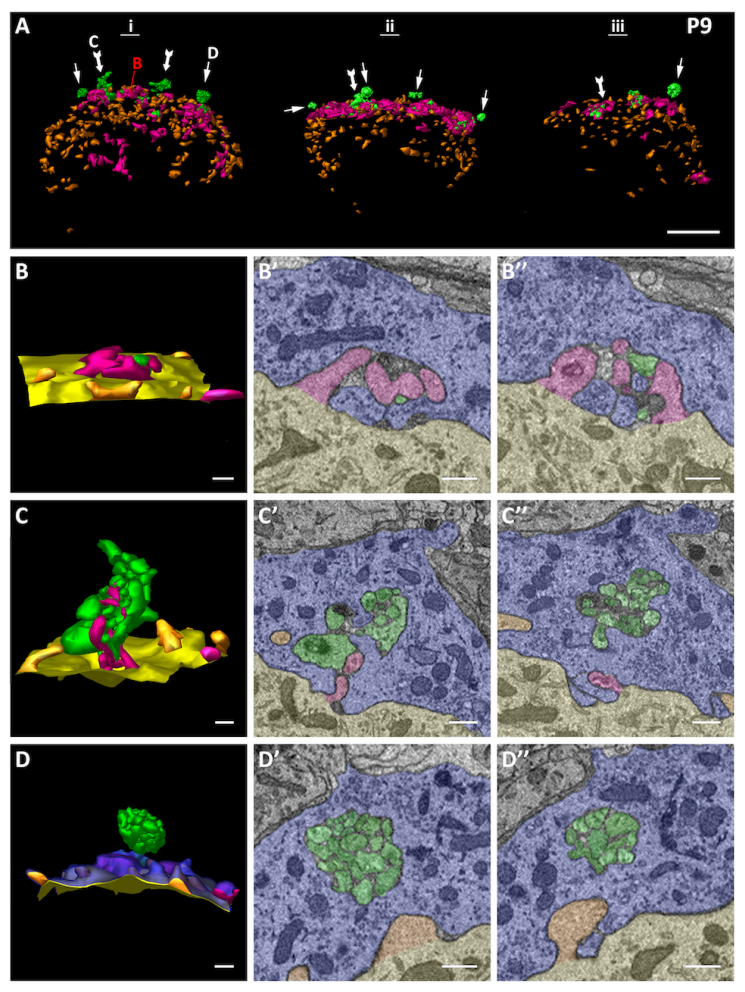

Chapter 3, Figure 10. Evidence in Support of Trans-Endocytosis of Type 2 Spines. A. Three of five $\mathrm{CH}$ terminals analyzed at P9 (i-iii) each show evidence of type 2 spine detachment (green) from the somatic surface and suspension of spines within the $\mathrm{CH}$. Spine mats in these $\mathrm{CHs}$ (i-iii: pink) can exist in various stages of type 2 spine detachment including "initial detachment", "partial detachment" (fletched arrows) or "full detachment" (unfletched arrow) as illustrated in B-D respectfully. B. Unlike earlier ages, P9 spine mats may include membrane bound inclusions that cannot be traced back to a cell of origin despite most type 2 spines maintaining a clear attachment to the somatic surface (gold) and the macrostructure of the mat (pink and green) exists in a vertically compressed morphology close to the somatic surface. B'-B', Two of three component type 2 spines (pink) from this spine mat are illustrated in cross-section about their attachment to the underlying soma (gold). C. Spine mats observed transitioning to a transendocytotic state is hallmarked by several distinctive features including: inclusion of attached type 2 spines of diminished caliber (pink); overall morphology of the spine mat extends vertically away from the somatic surface (gold), and many component spines exist detached from the somatic surface (green). C'-C', Selected 2D planes highlight the heterogeneity in spine size in a transitioning spine mat relative the large caliber type 2 spines evident where each is somatically attached (B'-B'). D, A trans-endocytosed spine mat is composed entirely of untethered spines (green) that reside wholly within the presynaptic terminal (detached mat in green resides above the presynaptic membrane shown in blue) while most other type 1 and 2 spines remain situated within invaginations of the presynaptic membrane (under the blue surface). D'-D", Detached spines in a trans-endocytosed mat are smaller than attached spines (see red spines immediately below) and the macrostructure is compressed into a sphere-like assemblage of component spines. Scale Bar: A, $5 \mu \mathrm{m}$; B-D”, $0.5 \mu \mathrm{m}$. 
Figures

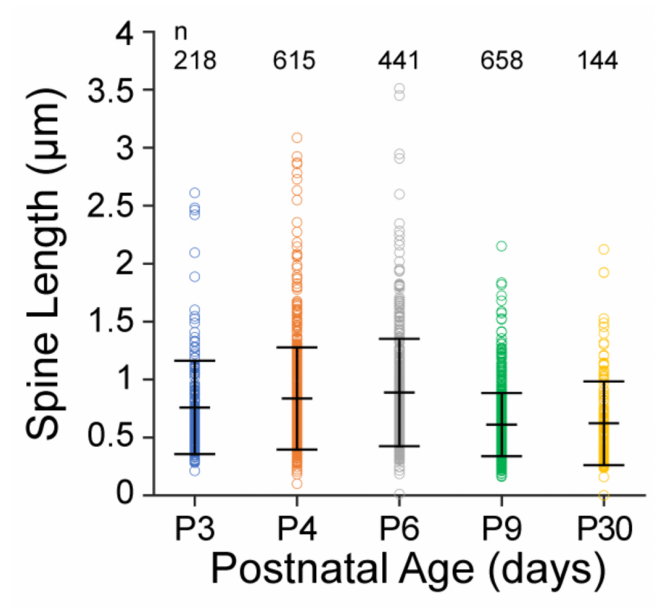

Chapter 3, Supplemental Figure 1. Comparison of single spine lengths across developmental ages. Mean values for single spine length per age increased from P3-6. Spine length declined by P9 and this shorter length is maintained until at least P30. Error bars are mean \pm SD; n-values for each age indicated on plot, total $n=2,076$. Statistically significant pairwise differences in length were found between all ages with the exceptions of P3-P4, P4-P6, and P9-P30 (Kruskal-Wallis $\mathrm{H}$-test with Nemenyi post-hoc test and Bonferroni correction (K-W). 
Figures

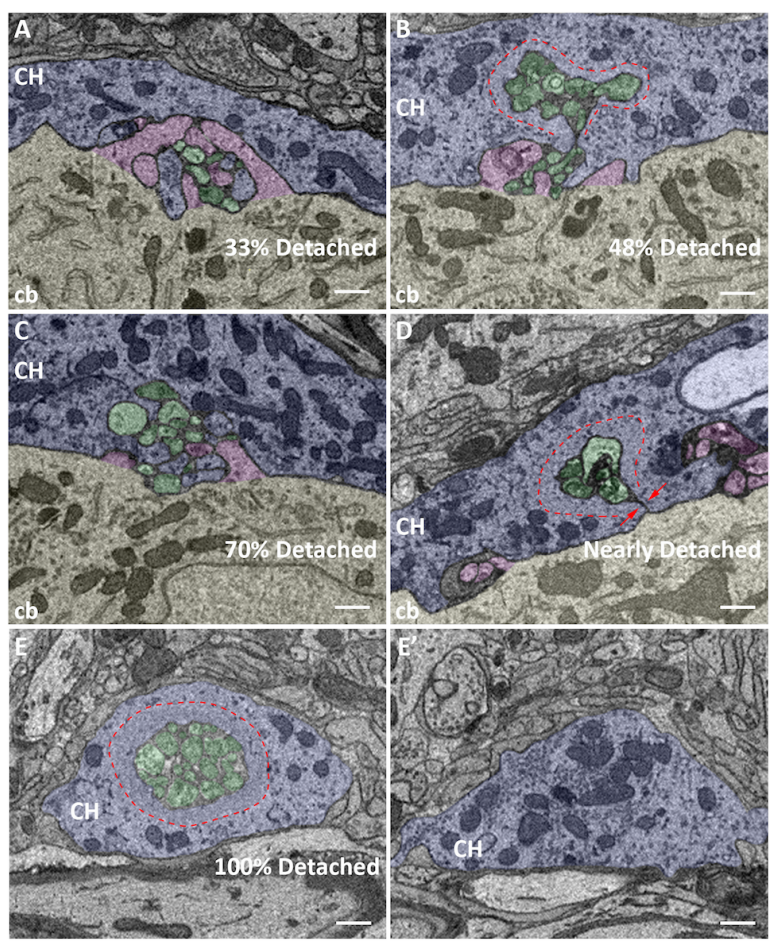

Chapter 3, Supplemental Figure 2. Transendocytosis of somatic spine mats and local degradation within the $\mathrm{CH}$. A-B', Two sections through a spine mat at P9 show several spines attached to the soma intermixed with apparent detached spines. Clusters of synaptic vesicles are located near the type 2 spines. In contrast, an organelle-free zone surrounds the detached elements within the $\mathrm{CH}$ (dotted red lines in $\mathbf{A}^{\prime}, \mathbf{B}^{\prime}$ ). C, Fully detached spines, with organelle free zone, located at axon junction with the $\mathrm{CH}$. This example may reflect spine mat degradation into a lysosome or multivesicular body. A'-C', Colored labels: blue, $\mathrm{CH}$; gold, cell body; type 1 spine, red (at left in B'); type 2 spines, pink; detached spines, green; organelle-free zone, dashed red outline. Scale Bar: A-C', $0.5 \mu \mathrm{m}$. 
Figures

\begin{tabular}{|c|c|c|c|c|c|c|c|c|c|}
\hline $\begin{array}{c}\text { Postnatal } \\
\text { Day (P) }\end{array}$ & $\begin{array}{c}\text { Number } \\
\text { of } \\
\text { Terminals }\end{array}$ & $\begin{array}{l}\text { Number of } \\
\text { Type } 1 \\
\text { Spines }\end{array}$ & $\begin{array}{c}\text { Type } 1 \\
\text { Spine } \\
\text { Length }(\mu \mathrm{m})\end{array}$ & $\begin{array}{c}\text { Type } 1 \text { Spine } \\
\text { Volume } \\
\left(\mu \mathrm{m}^{3}\right)\end{array}$ & $\begin{array}{c}\text { Per Type } 1 \\
\text { Spine Surface } \\
\text { Area }\left(\mu^{2}\right)\end{array}$ & $\begin{array}{c}\text { Per Terminal } \\
\text { Type } 1 \text { Spine } \\
\text { Surface Area } \\
\quad\left(\mu \mathrm{m}^{2}\right)\end{array}$ & $\begin{array}{c}\% \text { of } \\
\text { Terminal ASA } \\
\text { on Type } 1 \\
\text { Spines }\end{array}$ & $\begin{array}{l}\text { Number } \\
\text { of Type } \\
2 \text { Spines }\end{array}$ & $\begin{array}{c}\text { Number } \\
\text { of Spine } \\
\text { Mats }\end{array}$ \\
\hline \multicolumn{10}{|c|}{ Single Dominant Terminal } \\
\hline P3 & 6 & 125 & $0.792 \pm 0.42$ & $0.063 \pm 0.06$ & $1.07 \pm 0.69$ & $26.016 \pm 14.13$ & $10.7 \pm 4.4$ & 72 & 11 \\
\hline $\mathrm{P} 4$ & 9 & 436 & $0.866 \pm 0.57$ & $0.077 \pm 0.07$ & $1.168 \pm 0.77$ & $60.944 \pm 26.45$ & $20.5 \pm 4.8$ & 176 & 34 \\
\hline P6 & 3 & 247 & $0.967 \pm 0.51$ & $1.505 \pm 1.08$ & $1.505 \pm 1.08$ & $140.928 \pm 73.74$ & $37.4 \pm 10$ & 110 & 24 \\
\hline P9 & 5 & 658 & $0.609 \pm 0.27$ & $0.703 \pm 0.45$ & $0.703 \pm 0.45$ & $100.39 \pm 36.54$ & $17.2 \pm 4.1$ & 571 & 89 \\
\hline $\mathrm{P} 30$ & 5 & 144 & $0.621 \pm 0.36$ & $0.043 \pm 0.05$ & $0.76 \pm 0.62$ & $22.335 \pm 21.11$ & $4.63 \pm 3.6$ & 4 & 1 \\
\hline \multicolumn{10}{|c|}{ Largest Competing Terminal } \\
\hline P3 & 6 & 42 & $0.709 \pm 0.36$ & $0.067 \pm 0.07$ & $1.006 \pm 0.67$ & $7.045 \pm 4.36$ & $14.4 \pm 17$ & 0 & 0 \\
\hline $\mathrm{P} 4$ & 4 & 147 & $0.845 \pm 0.45$ & $0.078 \pm 0.07$ & $1.181 \pm 0.77$ & $43.392 \pm 17.75$ & $20.075 \pm 7.58$ & 98 & 7 \\
\hline P6 & 3 & 117 & $0.823 \pm 0.42$ & $0.078 \pm 0.08$ & $1.189 \pm 0.78$ & $46.383 \pm 6.79$ & $30.333 \pm 8.07$ & 43 & 7 \\
\hline \multicolumn{10}{|c|}{ Second Largest Competing Terminal } \\
\hline P3 & 6 & 29 & $0.673 \pm 0.21$ & $0.046 \pm 0.03$ & $0.797 \pm 0.37$ & $3.85 \pm 2.73$ & $8.417 \pm 7.25$ & 0 & 0 \\
\hline $\mathrm{P} 4$ & 4 & 37 & $0.701 \pm 0.31$ & $0.053 \pm 0.04$ & $0.912 \pm 0.48$ & $8.436 \pm 5.07$ & $8.978 \pm 2.653$ & 3 & 1 \\
\hline P6 & 3 & 85 & $0.741 \pm 0.28$ & $0.078 \pm 0.07$ & $1.204 \pm 0.71$ & $34.101 \pm 24.52$ & $26.75 \pm 10.285$ & 49 & 8 \\
\hline
\end{tabular}

Chapter 3, Table 1: Summary of morphological characteristics and terminal association of type 1 spines across all ages studied (P3-P30). Prevalence of type 2 spines and spine mats is also quantified. 
Figures

\begin{tabular}{|c|c|c|c|c|c|c|c|}
\hline & Terminal & $\begin{array}{c}\text { Terminal } \\
\text { ASA }\left(\mu \mathrm{m}^{2}\right)\end{array}$ & $\begin{array}{l}\text { Number of } \\
\text { Type } 1 \text { Spines }\end{array}$ & $\begin{array}{l}\text { Type } 1 \text { Spine } \\
\text { Length }(\mu \mathrm{m})\end{array}$ & $\begin{array}{l}\text { Type } 1 \text { Spine } \\
\text { Volume }\left(\mu \mathrm{m}^{3}\right)\end{array}$ & $\begin{array}{c}\text { Per Type } 1 \text { Spine } \\
\text { Surface Area } \\
\left(\mu \mathbf{m}^{2}\right)\end{array}$ & $\begin{array}{c}\% \text { of Terminal } \\
\text { ASA on Type } 1 \\
\text { Spines } \\
\end{array}$ \\
\hline \multirow{12}{*}{$\mathbf{P 3}$} & $1 \mathrm{a}$ & 68.8 & 32 & $0.73 \pm 0.4$ & $0.068 \pm 0.05$ & $1.05 \pm 0.6$ & 48.8 \\
\hline & $1 \mathrm{~b}$ & 33.5 & 7 & $0.60 \pm 0.2$ & $0.057 \pm 0.05$ & $0.88 \pm 0.5$ & 18.4 \\
\hline & $2 a$ & 68.5 & 5 & $0.77 \pm 0.3$ & $0.073 \pm 0.07$ & $1.04 \pm 0.7$ & 7.6 \\
\hline & $2 \mathrm{~b}$ & 42.5 & 8 & $0.65 \pm 0.3$ & $0.049 \pm 0.04$ & $0.84 \pm 0.4$ & 15.8 \\
\hline & $3 a$ & 57.3 & 5 & $0.67 \pm 0.4$ & $0.086 \pm 0.20$ & $0.93 \pm 1.1$ & 8.1 \\
\hline & $3 \mathrm{~b}$ & 35.5 & 2 & $0.64 \pm 0.01$ & $0.036 \pm 0.02$ & $0.68 \pm 0.2$ & 3.8 \\
\hline & $4 a$ & 142 & 7 & $0.66 \pm 0.4$ & $0.052 \pm 0.03$ & $0.90 \pm 0.5$ & 2.2 \\
\hline & $4 \mathrm{~b}$ & 121 & 7 & $0.71 \pm 0.2$ & $0.042 \pm 0.02$ & $0.77 \pm 0.3$ & 4.4 \\
\hline & $5 a$ & 53.4 & 5 & $0.73 \pm 0.1$ & $0.044 \pm 0.02$ & $0.89 \pm 0.3$ & 8.4 \\
\hline & $5 b$ & 38.1 & 0 & 0 & 0 & 0 & 0 \\
\hline & $6 a$ & 140 & 15 & $0.83 \pm 0.6$ & $0.064 \pm 0.07$ & $1.05 \pm 0.7$ & 11.3 \\
\hline & $6 \mathrm{~b}$ & 43.2 & 5 & $0.62 \pm 0.2$ & $0.034 \pm 0.02$ & $0.70 \pm 0.3$ & 8.1 \\
\hline \multirow{8}{*}{ P4 } & $1 \mathrm{a}$ & 259 & 43 & $0.68 \pm 0.3$ & $0.048 \pm 0.04$ & $0.84 \pm 0.5$ & 13.3 \\
\hline & $1 \mathrm{~b}$ & 74.6 & 7 & $0.53 \pm 0.2$ & $0.028 \pm 0.01$ & $0.58 \pm 0.2$ & 5.4 \\
\hline & $2 a$ & 254 & 42 & $1.18 \pm 1.1$ & $0.099 \pm 0.09$ & $1.35 \pm 0.9$ & 21.9 \\
\hline & $2 \mathrm{~b}$ & 84.3 & 11 & $0.71 \pm 0.3$ & $0.042 \pm 0.03$ & $0.79 \pm 0.4$ & 10.2 \\
\hline & $3 a$ & 117 & 17 & $0.76 \pm 0.3$ & $0.076 \pm 0.05$ & $1.17 \pm 0.7$ & 15.1 \\
\hline & $3 b$ & 48.2 & 5 & $1.12 \pm 0.6$ & $0.070 \pm 0.05$ & $1.12 \pm 0.6$ & 11.6 \\
\hline & $4 a$ & 193.4 & 45 & $0.86 \pm 0.5$ & $0.089 \pm 0.07$ & $1.29 \pm 0.8$ & 30 \\
\hline & $4 \mathrm{~b}$ & 178.7 & 14 & $0.82 \pm 0.4$ & $0.069 \pm 0.04$ & $1.16 \pm 0.5$ & 7.8 \\
\hline \multirow{6}{*}{ P6 } & $1 \mathrm{a}$ & 150.9 & 35 & $1.24 \pm 1.6$ & $0.083 \pm 0.10$ & $1.25 \pm 1.1$ & 28.9 \\
\hline & $1 \mathrm{~b}$ & 131.4 & 34 & $0.75 \pm 0.3$ & $0.066 \pm 0.20$ & $1.06 \pm 0.5$ & 27.4 \\
\hline & $2 a$ & 240.8 & 47 & $0.78 \pm 0.3$ & $0.068 \pm 0.06$ & $1.10 \pm 0.6$ & 21.5 \\
\hline & $2 b$ & 157.7 & 41 & $0.78 \pm 0.3$ & $0.089 \pm 0.08$ & $1.31 \pm 0.9$ & 34 \\
\hline & $3 a$ & 104 & 30 & $0.85 \pm 0.4$ & $0.085 \pm 0.06$ & $1.29 \pm 0.6$ & 37.2 \\
\hline & $3 \mathrm{~b}$ & 50.9 & 7 & $0.70 \pm 0.2$ & $0.073 \pm 0.03$ & $1.14 \pm 0.5$ & 15.6 \\
\hline
\end{tabular}

Chapter 3, Table 2: Pairwise comparison of morphological characteristics and terminal associations of the two largest competing terminals per cell. 
Figures

\begin{tabular}{|ccc|}
\hline Group 1 & Group 2 & p-value \\
P3 & P4 & 0.105782 \\
P3 & P6 & $\mathbf{0 . 0 0 0 6 8 1}$ \\
P3 & P9 & $\mathbf{4 . 8 1 E - 0 6}$ \\
P3 & P30 & $\mathbf{0 . 0 0 0 2 5 6}$ \\
P4 & P6 & 0.170715 \\
P4 & P9 & $\mathbf{3 . 7 5 E}-14$ \\
P4 & P30 & $\mathbf{8 . 5 5 E - 1 1}$ \\
P6 & P9 & $\mathbf{3 . 7 5 E - 1 4}$ \\
P6 & P30 & $\mathbf{1 . 5 8 E - 1 4}$ \\
P9 & P30 & 0.972052 \\
\hline
\end{tabular}

Chapter 3, Supplemental Table 1: Pairwise statistical comparison in single spine lengths between developmental ages using the Kruskal-Wallis H-test with Nemenyi post-hoc test and Bonferroni correction. All pairwise comparisons were statistically significant except for P3-P4, P4-P6, and P9-P30. 

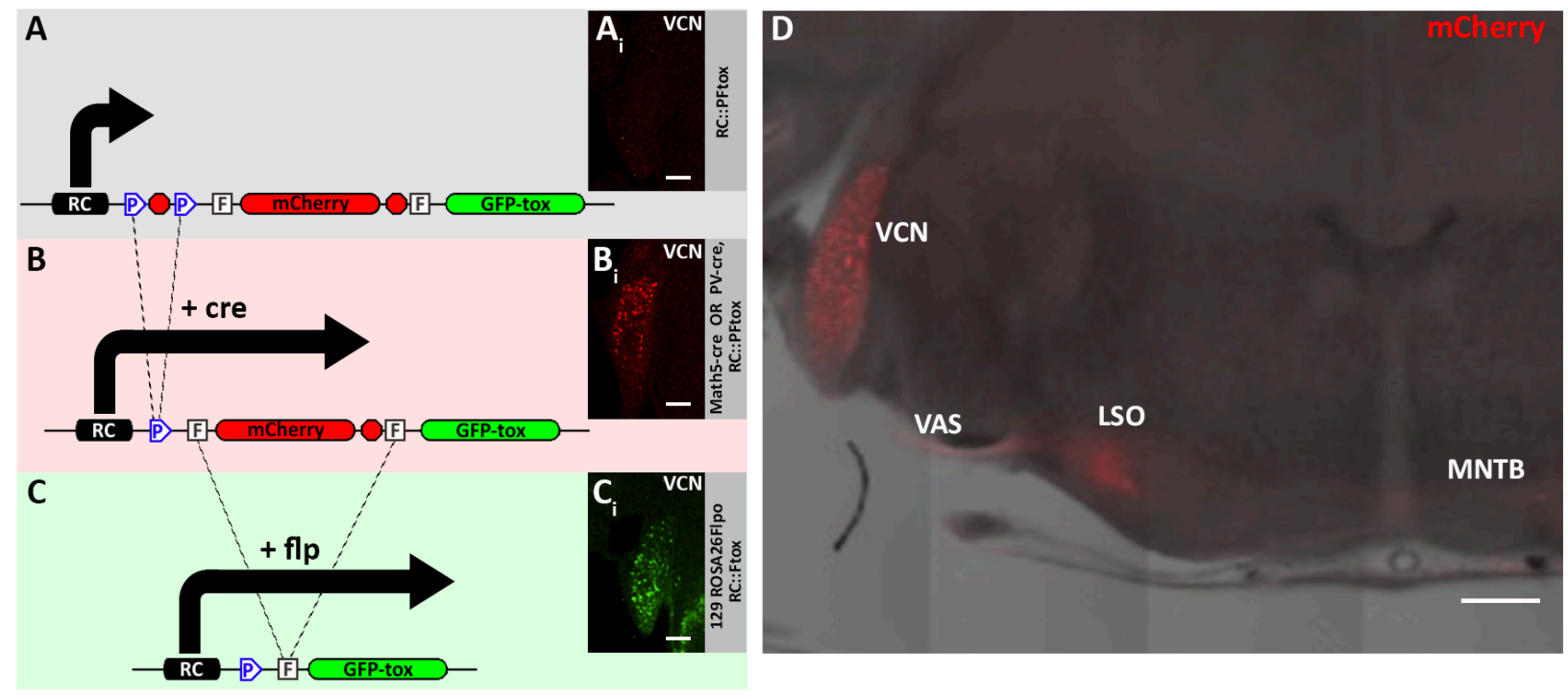

Chapter 4, Figure 1: RC::PFtox provides conditional expression of a GFPtox fusion protein for 'synaptic silencing'. A, RC::PFtox contains two stop cassettes, one flanked by directly oriented loxP sites (P-pentagons) and the other, by FRT sites (F-squares) that prevent expression of mCherry and GFPtox $\left(\mathbf{A}_{\mathbf{i}}\right)$. B, Cre-mediated stop cassette recombination permits mCherry expression $\left(\mathbf{B}_{\mathbf{i}}\right)$ while GFPtox expression is prevented by the remaining FRT-flanked stop cassette. $\mathbf{C}$, Cre- and Flp-mediated recombination results in constitutive GFPtox expression $\left(\mathbf{C}_{\mathbf{i}}\right)$. D, RC::PFtox crossed with PV-cre results in strong mCherry expression in neurons of the cochlear nucleus in postnatal animals with clear labeling of projections along the ventral acoustic stria (VAS) towards targets in the ipsilateral lateral superior olive (LSO) and the contralateral medial nucleus of the trapezoid body (MNTB). Scale Bar: $\mathbf{A}_{\mathbf{i}}-\mathbf{C}_{\mathbf{i}}, 250 \mu \mathrm{m} ; \mathbf{D}, 500 \mu \mathrm{m}$. 

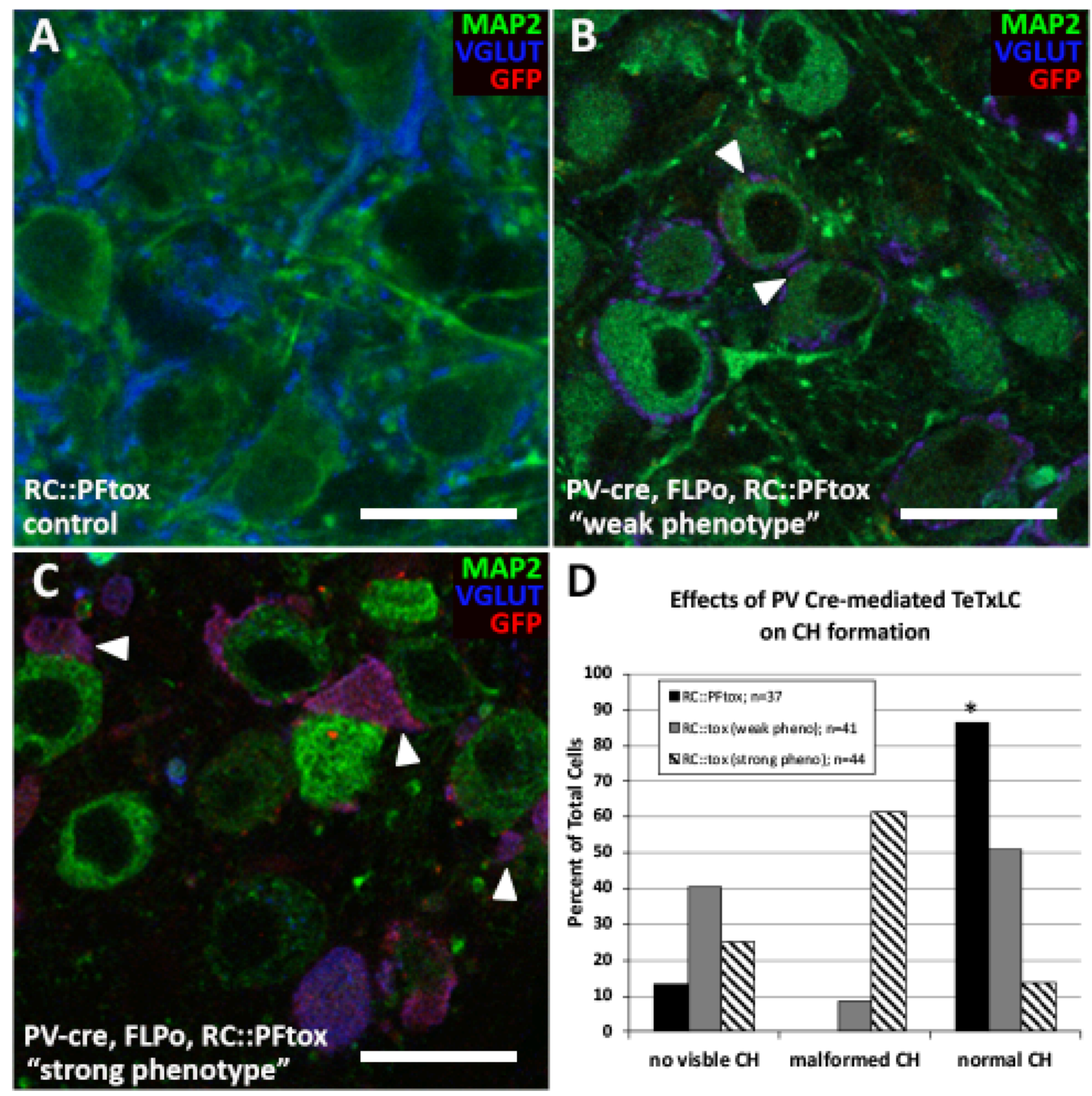

Chapter 4, Figure 2: Resultant litters of RC::tox animals from a PV background exhibit variable phenotypes revealed by disparate motor impairment and impaired $\mathrm{CH}$ growth by $\mathrm{P} 9$ revealed by immunohistochemical analysis. A, Homozygous RC::PFtox animals produce stereotypical cup-like innervation to the developing PC of the MNTB, with contiguous VGLUT+ compartments that envelop the PC soma. B, 'Weakly phenotypic' RC::tox animals produce fewer cup-like terminals in the developing MNTB. C, 'Strongly phenotypic' RC::tox animals exhibited gross malformations in $\mathrm{CH}$ structure relative to controls. (B-C), Cell bodies are labeled with anti-microtubule associated protein 2 (MAP2, green), presynaptic terminals are labeled with anti-vesicular glutamate transporters 1 and 2 (VGLUT, blue), and paradoxically anti-GFP (red). Arrowheads indicate overlapping GFP and VGLUT signal is restricted to presynaptic compartments. D, Reconstruction of the presynaptic terminals revealed inhibition of synaptic activity significantly reduced the formation of stereotypical $\mathrm{CH}$ terminals. Scale Bar: A-C, 20 $\mu \mathrm{m}$. 

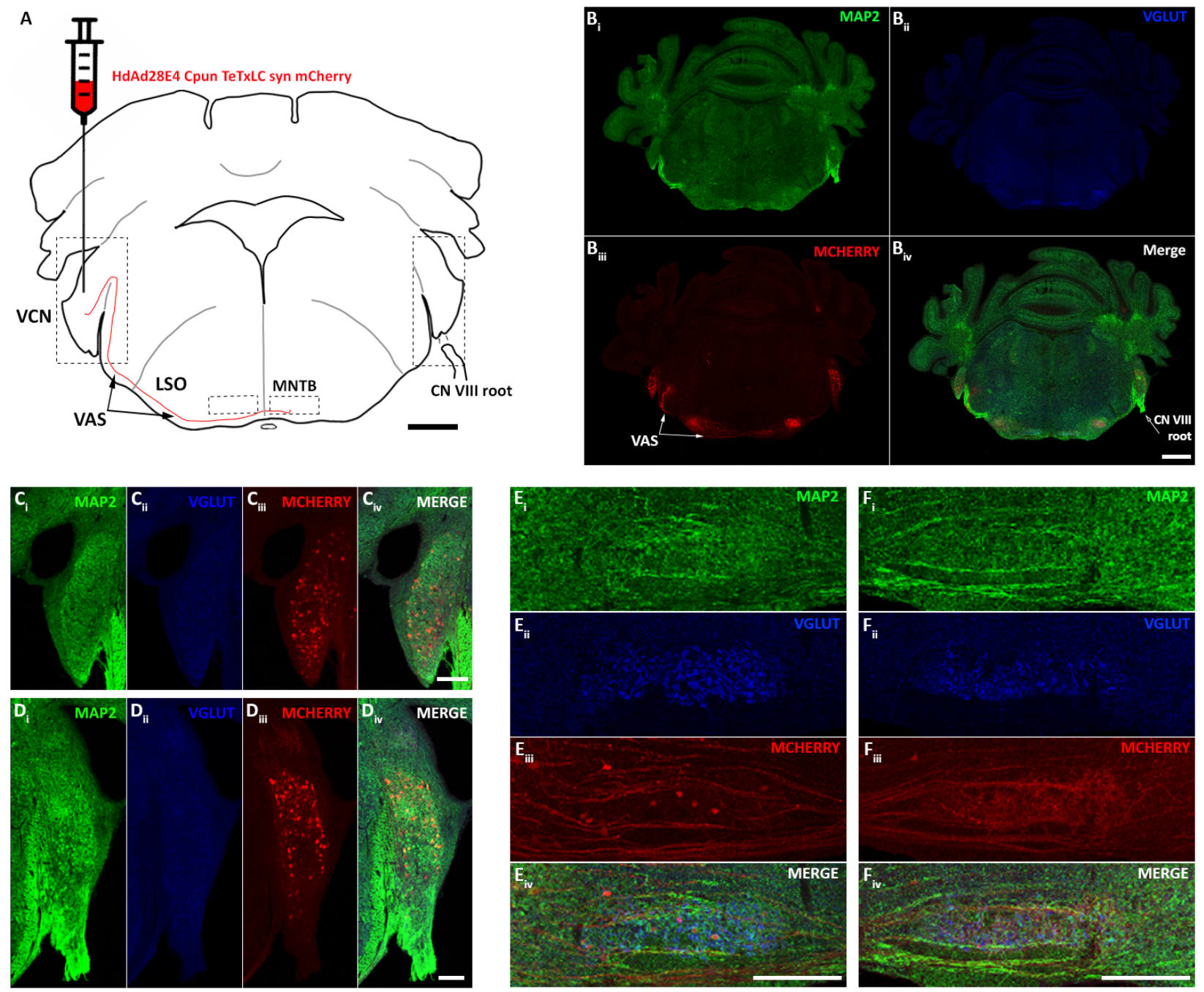

Chapter 4, Figure 3: Unilateral inhibition of synaptic activity ahead of the growth of the CH. A, On the day of birth, the left VCN was targeted for stereotaxic delivery of a replication incompetent adenovirus for rapid expression of the enzymatically active light chain of tetanus toxin for selective abolishment of synaptic vesicle release (HdAd28E4 Cpun TeTxLC syn mCherry). B-Biv, Within days of transfection, robust mCherry labeling suggests strong TeTxLC expression in labeled cells. C-Civ, By P5, cells across the injected VCN express mCherry. DDiv, Projections from the contralateral VCN were also retrogradely transfected. E-Eiv, Despite bilateral expression of mCherry, no labeled CHs were observed in the MNTB ipsilateral to the injection site. F-Fiv, Numerous mCherry labeled endings were found in the MNTB contralateral to the injection site. B-F, Cell bodies are labeled with anti-microtubule associated protein 2 (MAP2, green), presynaptic terminals are labeled with anti-vesicular glutamate transporters 1 and 2 (VGLUT, blue), and presynaptic afferents are shown in red (mCherry). Scale Bar: A, 200 $\mu \mathrm{m} ; \mathbf{B}, 500 \mu \mathrm{m} ; \mathbf{C}-\mathbf{F}, 250 \mu \mathrm{m}$. 

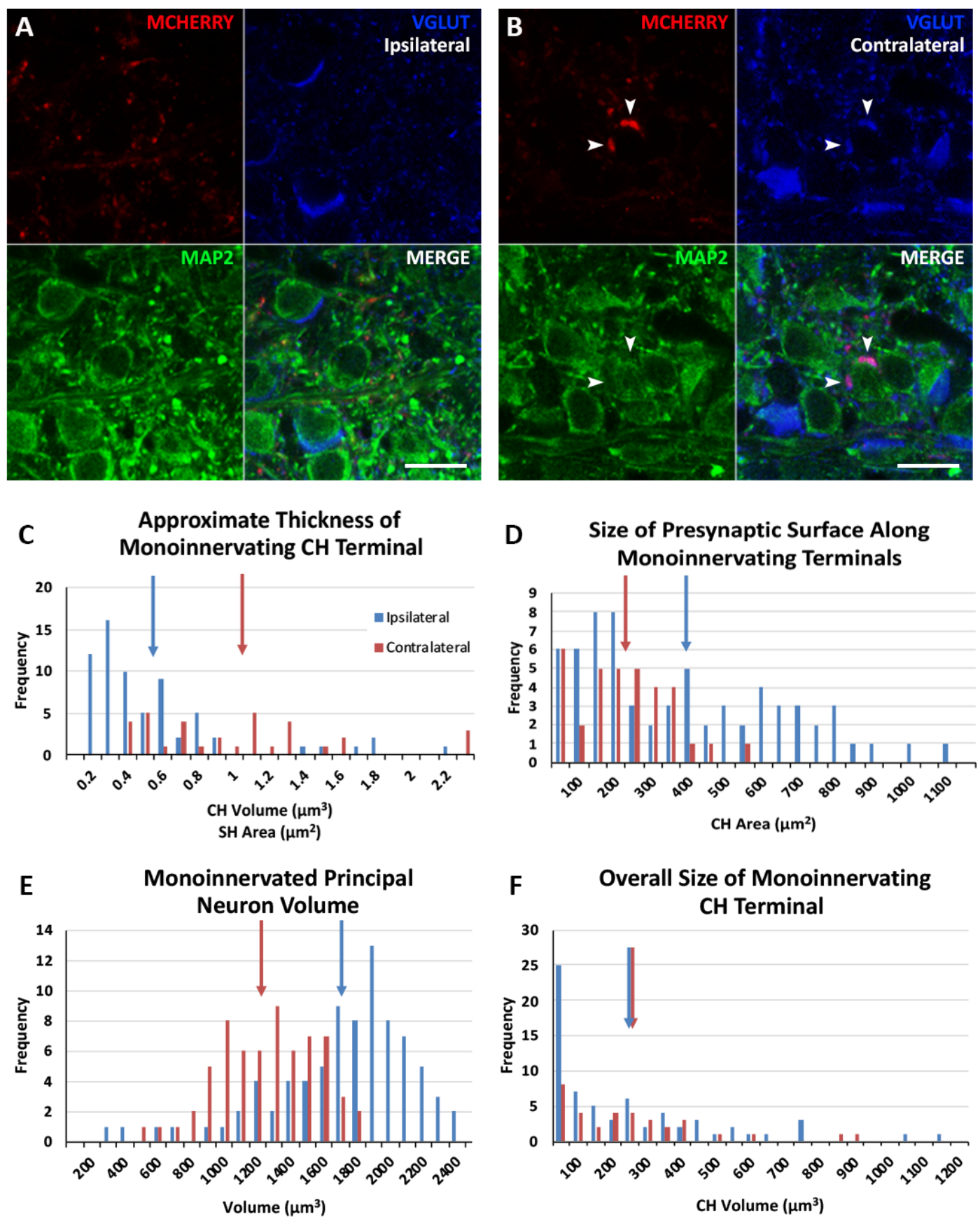

Chapter 4, Figure 3: Unilateral inhibition of synaptic activity provided within animal controls for the evaluation of $\mathrm{CH}$ growth downstream of 'synaptic silencing'. A, Non-trnsfected afferents to the ipsilateral MNTB produced cup-like terminals free of mCherry expression although punctate mCherry labeling likely represent commissural GBC axons from the transfected VCN. $\mathbf{B}$, Within the contralateral MNTB, relative to the stereotaxic injection, enlarged mCherry+ signal colocalizes with VGLUT labeling suggesting the formation of at least some enlarged terminals. C-F, Reconstruction of the morphology of the $\mathrm{CH}$ in each MNTB were evaluated to assess the effect of TeTxLC expression on terminal morphology. $\mathbf{C}$, Silenced terminals formed bloated terminals that almost double the thickness of the steotypically thin and cup-like terminals 
in the ipsilateral MNTB (TeTxLC-). D, Silenced terminals occupied nearly half the somatic area occupied by CHs in the ipsilateral MNTB (TeTxLC-). E, Abolishment of presynaptic activity resulted in a reduction in postsynaptic cell volume. F, Despite silenced terminals inability to form a stereotypical morphology, no difference in terminal volume was detected between brain hemispheres. A-B, Cell bodies are labeled with anti-microtubule associated protein 2 (MAP2, green), presynaptic terminals are labeled with anti-vesicular glutamate transporters 1 and 2 (VGLUT, blue), and presynaptic afferents are shown in red (mCherry). Scale Bar: A-B, $20 \mu \mathrm{m}$. 


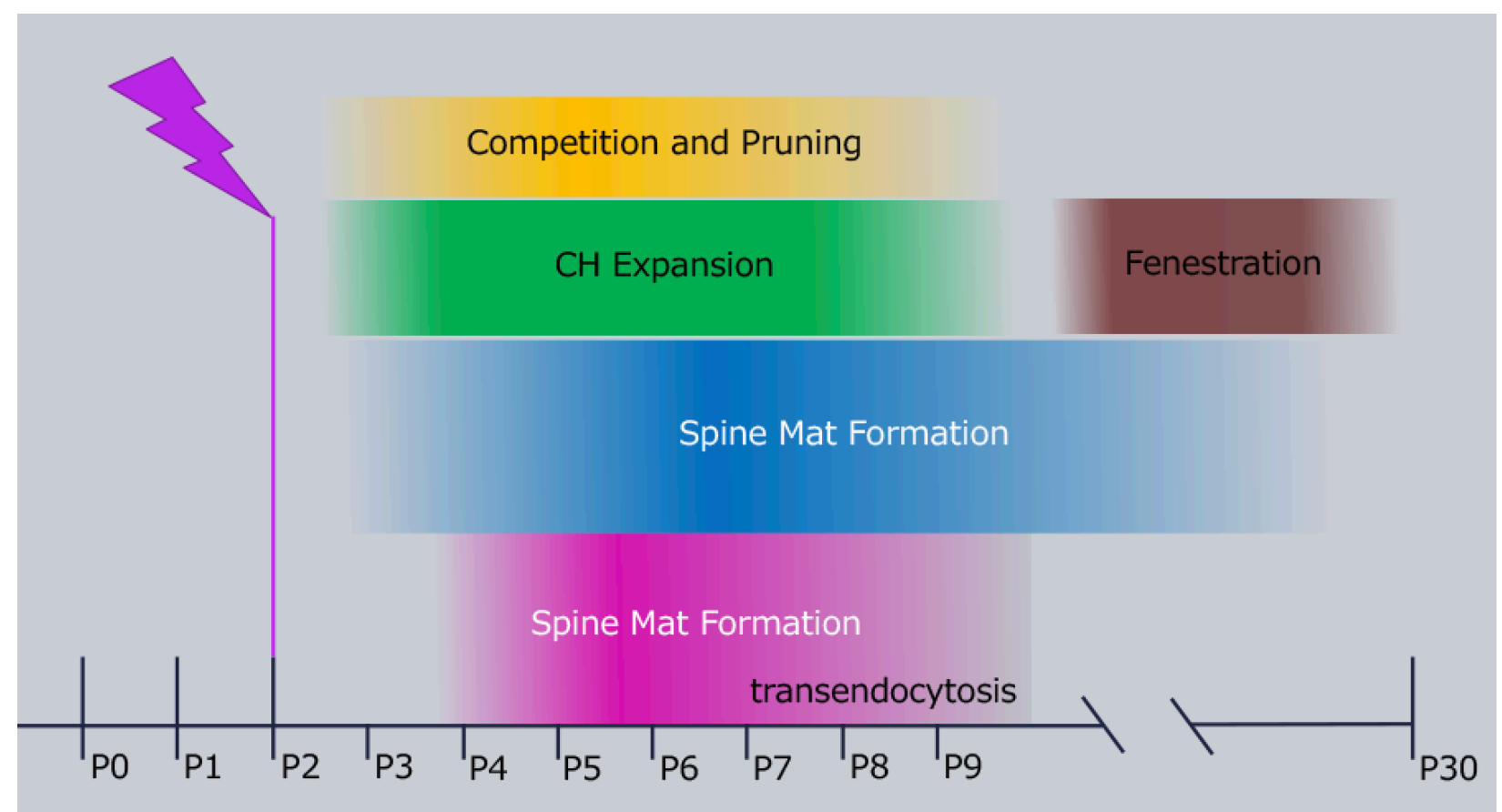

Chapter 5, Figure 1: Summary of the major events pertaining to the growth of the CH. 